\title{
Symmetry Breaking and Pattern Selection in Models of Visual Development
}

\section{Dissertation}

zur Erlangung des Doktorgrades

der Mathematisch-Naturwissenschaftlichen Fakultäten der Georg-August-Universität zu Göttingen

vorgelegt von

Lars Reichl

aus Sinsheim

Göttingen 2010 
Referent:

Koreferent:

Tag der mündlichen Prüfung:
Prof. Dr. Theo Geisel

Prof. Dr. Ulrich Parlitz

18.05.2010 


\section{Contents}

1. Introduction 1

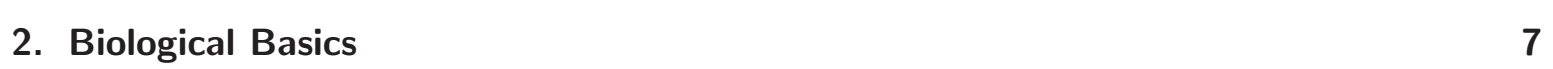

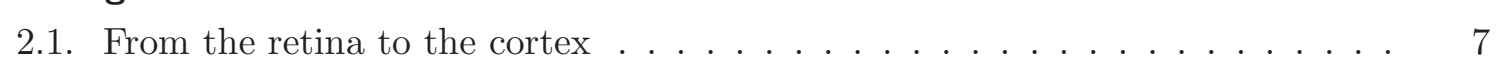

2.2. Maps in the visual cortex . . . . . . . . . . . . . . . 7

2.2.1. Ocular dominance maps . . . . . . . . . . . . . . . . . . . . . . . . . .

2.2.2. Orientation preference maps . . . . . . . . . . . . . . . 9

2.3. Long-range connections . . . . . . . . . . . . . . . . . . . 12

2.4. Geometric relationships . . . . . . . . . . . . . . . . . . . 12

2.5. Variation across different species . . . . . . . . . . . . . . . 13

2.6. Development of cortical maps . . . . . . . . . . . . . . . . . . . . 14

3. Model Basics 15

3.1. A generalized Swift-Hohenberg model . . . . . . . . . . . . . . 15

3.2. Inter-map coupling: A svmmetrv-based analvsis . . . . . . . . . . . . . . 17

3.3. Analvtical and numerical methods . . . . . . . . . . . . . . . 22

3.4. Tracking and counting pinwheels . . . . . . . . . . . . . . . 22

4. Pinwheel Stabilization by Inter-Map Coupling 25

4.1. Introduction . . . . . . . . . . . . . . . . . . . . . . 25

4.2. The transition from OD stripes to OD blobs . . . . . . . . . . . 26

4.2.1. Mapping to a dvnamics with a quadratic nonlinearity . . . . . . . . 26

4.2.2. Amplitude equations for OD patterns . . . . . . . . . . . . 27

4.2.3. Stationarv solutions . . . . . . . . . . . . . . . . . 30

4.2.4. Linear stabilitv analvsis for OD patterns . . . . . . . . . . . . . . 30

4.2.5. Contralateral eve fraction . . . . . . . . . . . . . . . 32

4.3. Coupled amplitude equations . . . . . . . . . . . . . . . 33

4.4. Inter-map coupling: Low order coupling terms . . . . . . . . . . . . . . 38

4.4.1. Product-type energv $U=\alpha o^{2}|z|^{2} \ldots \ldots \ldots$. . . . . . . . . 39

4.4.2. Gradient-tvpe energv $U=\beta|\nabla o \nabla z|^{2} \ldots \ldots \ldots$. . . . . . . . 47

4.5. Inter-map coupling: Higher order coupling terms . . . . . . . . . . . 50

4.5.1. Product-tvpe energv $U=\tau o^{4}|z|^{4} \ldots \ldots \ldots$. . . . . . . . . 51

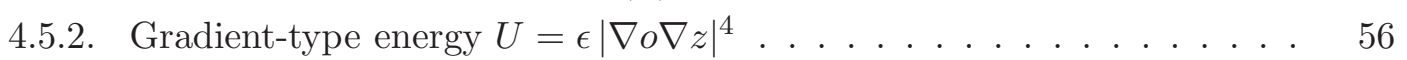

4.6. Numerical analvsis of pinwheel crystallization . . . . . . . . . . . . . . 61

4.6.1. Pinwheel kinetics . . . . . . . . . . . . . . . . . . . . 62 
4.6.2. Detuning $\mathrm{OD}$ and $\mathrm{OP}$ wavelengths . . . . . . . . . . . . . 65

4.6.3. Higher feature dimensions . . . . . . . . . . . . . . . 67

4.7. Discussion . . . . . . . . . . . . . . . . . . . . 71

5. Shaping the Lavout of OD Maps by Inter-Map Coupling 75

5.1. Introduction . . . . . . . . . . . . . . . . . . . . . . 75

5.2. Permutation svmmetrv . . . . . . . . . . . . . . . 76

5.3. The uncoupled dvnamics . . . . . . . . . . . . . . . . . . . . . 76

5.4. Low order coupling energies . . . . . . . . . . . . . . . . . 79

5.5. Higher order coupling energies . . . . . . . . . . . . . . . . . . 81

5.5.1. Phase equations . . . . . . . . . . . . . . . 82

5.6. Uniform solutions . . . . . . . . . . . . . . . . . . . . 83

5.6.1. Measures of uniformity . . . . . . . . . . . . . . . 85

5.6.2. Stability properties . . . . . . . . . . . . . . . 86

5.7. Including the backreaction . . . . . . . . . . . . . . . . . . 89

5.8. Geometric relationships . . . . . . . . . . . . . . . . . . . 90

5.9. Numerical analvsis of the coupled dynamics . . . . . . . . . . . . . . 93

5.9.1. Pinwheel kinetics . . . . . . . . . . . . . . . . . . 94

5.9.2. Time evolution of the OD lavout . . . . . . . . . . . . . 97

5.9.3. The OD lavout of macaques: From patches to stripes . . . . . . . . 97

5.10. Discussion . . . . . . . . . . . . . . . . . 100

6. Pattern Selection Far from Threshold 103

6.1. A preliminary remark . . . . . . . . . . . . . . . . . . . . . 103

6.2. Introduction . . . . . . . . . . . . . . . . . . . . . . 103

6.3. Amplitude equations - Higher order corrections . . . . . . . . . . . . . . . 104

6.3.1. Examples: Stripes and squares . . . . . . . . . . . . . . . . . . . . 109

6.4. Phase relations . . . . . . . . . . . . . . . . . . . . . 110

6.4.1. Phase relations in essentially complex planforms . . . . . . . . . . . . 110

6.4.2. The growth of opposite modes . . . . . . . . . . . . . . . . . . . . 111

6.4.3. Phase relations in numerical simulations . . . . . . . . . . . . . . . 112

6.5. Stationarv amplitudes . . . . . . . . . . . . . . . . . . 115

6.6. Pinwheel densities - Near and far from threshold . . . . . . . . . . . . . . . 117

6.7. Lifting the planform degeneracv . . . . . . . . . . . . . . . . . . 120

6.8. The influence of non-resonant modes . . . . . . . . . . . . . . . . . . . 122

6.9. Stabilitv borders . . . . . . . . . . . . . . . . 126

6.9.1. Intrinsic stability . . . . . . . . . . . . . . . . . . 127

6.9.2. Extrinsic stability . . . . . . . . . . . . . . . . 127

6.9.3. The question of convergence . . . . . . . . . . . . . . . . . 130

6.10. Discussion . . . . . . . . . . . . . . . . . . . 130 
7. Pattern Selection with Broken Permutation Symmetry 133

7.1. Breaking the permutation symmetry . . . . . . . . . . . . . . 133

7.2. The extended solution set . . . . . . . . . . . . . . . . 137

7.2.1. Essentially complex planforms . . . . . . . . . . . . . . 137

7.2.2. Circular phase progression solutions . . . . . . . . . . . . . . . 140

7.2.3. Binarv phase planforms . . . . . . . . . . . . . . . . 142

7.3. Pinwheel densities with broken symmetry . . . . . . . . . . . . . 145

7.4. Lifting the planform degeneracy at leading order . . . . . . . . . . 146

7.5. Stabilitv borders . . . . . . . . . . . . . . . . . 147

7.6. Including higher order corrections . . . . . . . . . . . . . . . . 154

7.7. Numerical analysis of pattern selection . . . . . . . . . . . . . . . . . . . . . . . . . . . . . . . . . . . . . . . . . .

7.8. Discussion . . . . . . . . . . . . . . . . . . . . . . . . . 159

$\begin{array}{ll}\text { 8. Conclusion } & 161\end{array}$

9. Notation guide $\quad 165$

$\begin{array}{ll}\text { A. Appendix } & 167\end{array}$

A.1. Numerical integration method . . . . . . . . . . . . . . . . . 167

A.2. Coupling coefficients I . . . . . . . . . . . . . . . . 169

A.3. Coupling coefficients II . . . . . . . . . . . . . . . . . . . . . 170

A.4. Calculation of the bandedness . . . . . . . . . . . . . . 171

A.5. Fifth order coupling coefficients . . . . . . . . . . . . . . . . . . . 172

$\begin{array}{ll}\text { Bibliography } & 177\end{array}$

\begin{tabular}{ll}
\hline Acknowledgments & 187
\end{tabular}

$\begin{array}{lr}\text { Curriculum vitae } & 189\end{array}$ 


\section{Introduction}

Pattern formation is observed in abundance, including animal coat markings [1], convection experiments [2 4], laser beams [5], and Faraday waves [6, 7]. Another prominent pattern formation process is the ability of the brain to form spatial representations of sensory information. Well known examples are the topographic representation of the body surface in the somatosensory areas [8] and acoustic maps in the auditory area 9]. The diversity of such patterns is not only fascinating to observe in nature but also to study using concepts from physics. In this thesis we study the formation of sensory representations in the visual cortex. Neurons in the primary visual cortex are selective to the complex structure of a natural scene, including visual field position, contour orientation, ocular dominance, direction of motion, and spatial frequency. In many mammals the preferred orientation of visual cortical neurons were found to form a complex two dimensional pattern, called the orientation preference (OP) map. Another important cortical organization structure is ocular dominance (OD) i.e. the tendency to prefer visual input from one eye over the other. Response properties in these maps are arranged in repetitive modules of a typical length called hypercolumns. OP and OD maps exist in many species with very different living conditions including humans [10,11]. How do these cortical maps emerge during development? It seems plausible to believe that evolution has designed the visual system of mammals such as to form a very efficient internal representation of the outside world. There is abundant evidence that neural activity plays a role in organizing the connections among neurons during development. What is an appropriate model for the development of visual cortical maps that captures the observed phenomenology? If the refinement of cortical architecture follows an optimization strategy this process can be interpreted as the convergence towards a ground state of some energy functional. However, the energy functional the brain tries to minimize is unknown. Established models, that propose a specific optimization principle, raise several questions which we discuss in the current thesis: Is there a model independent approach to analyze the simultaneous development of cortical maps? What are the genuine ground states of such energy functionals and how can they be calculated analytically? How do different optimization principles impact on the predicted map structure and conversely what can be learned about the optimized energy from observation of map structures? Can the spatially irregular organization of cortical functional maps be explained by frustration resulting from the joint optimization of multiple maps?

The description of cortical map development on the level of individual neurons and synapses is very complex considering their enormous number. For instance, there are on average about $10^{5}$ neurons and $10^{9}$ synapses in each $\mathrm{mm}^{3}$ of cortex [12]. Instead we can describe map 
formation on a mesoscopic level. Here, relatively simple effective relations such as symmetries of the underlying system rule the map formation. Some plausible symmetries have been proposed to hold for cortical maps which can help to specify the dynamics of cortical maps, for instance the invariance under spatial translations and rotations or a global shift of orientation preference. However, even if a model for cortical development results from simple rules and assumptions its analytical tractability can be quite low. In the current thesis we present a dynamical systems approach for analyzing cortical map development. We use a perturbation method called weakly nonlinear analysis which is used in many analogous physical systems $[2,3,6,13,14$. This method reduces the dimension of the system and leads to amplitude equations as an approximate description of the dynamics near the pattern forming instability. These amplitude equations are usually truncated at the leading order of the expansion. In most cases this yields already a good description of the underlying field dynamics and allows for an analytical treatment of the dynamics.

\section{The problem of pinwheel stability}

It is important to find quantifiable criteria of equivalences between models of visual cortical development and those maps actually found in the visual cortex. It turns out that the occurrence of so called pinwheels is such an appropriate measure. OP maps are characterized by pinwheels, regions in which columns preferring all possible orientations are organized around a common center in a radial fashion [15]. Pinwheels are initially generated in the visual cortex at the time of eye opening and remain present during all stages of visual development. Pinwheels occur numerously in the primary visual cortex. A recent study found that diverse species exhibit a universal pinwheel density of about three pinwheels per hypercolumn [16]. However, the functional role of pinwheels remains unclear. Does the existence of pinwheels influence visual processing? Near pinwheels neurons with dissimilar orientation preferences are closely located. The existence of pinwheels thus might reduce the wiring length between neurons and therefore improve information processing [17]. However, many theoretical models of visual cortical development have failed to produce OP maps possessing stable pinwheels. Simulations of various models have demonstrated that initially pinwheel rich states, generated by spontaneous symmetry breaking, can decay towards a pinwheel sparse pattern of OP stripes, a process known as spontaneous pinwheel annihilation [17 20]. The question of pinwheel stability in OP maps is thus an unsolved problem in theoretical neuroscience. To solve this problem we need a mechanism that stabilizes pinwheels. There are currently two hypotheses for the stabilization of pinwheels which will both be examined in this thesis. According to the first hypothesis, pinwheels are stabilized by interactions among different columnar systems. According to the second hypothesis, long-range intracortical interactions can lead to pinwheel stabilization.

\section{Stabilization by map interactions}

One hypothesis for pinwheel stabilization is suggested by the observed spatial relationships between different visual cortical maps. These relationships, such as the tendency of isoorientation lines to intersect OD borders perpendicularly or the tendency for pinwheels to 
be located at OD extrema, have been proposed to reflect an optimization principle, e.g. a compromise between stimulus coverage and continuity [21 24] or a wiring length minimization [17, 25]. Hubel and Wiesel suggested in their 'Ice cube' model a simple organization structure of OD and OP maps [26]. They proposed that OD bands should intersect isoorientation bands at right angles such that a single hypercolumn would cover all possible stimulus combinations. This model ignores the existence of pinwheels and the spatial disorder obvious in the patterns. Thereafter, the influence of OD segregation on OP maps has been studied in a series of models [18, 20, 24, 27, -38]. In many of these models it is expected that the inter-map coupling preferentially locates pinwheels at OD extrema and prevents them from being at OD borders. Pinwheels thus cannot cross OD borders, as it would increase the energy of the system. Pinwheels are topological defects and therefore can annihilate only in pairs. This mechanism thus may prevent pinwheels from annihilating each other and one may expect that a strong OD segregation impedes pinwheel annihilation. In this thesis we will show that this is not true for a general OD layout and that additional requirements such as a broken left-right eye symmetry have to be fulfilled.

All these models rely on numerical simulations to study the time evolution of map development. Although several numerical studies [18,20] showed that the annihilation of pinwheels is impeded due to OD segregation it remains unclear whether pinwheel annihilation is actually stopped and the remaining pinwheels in the pattern are stable or whether this is just a transient state and a further pinwheel annihilation leads to a OP stripe pattern. Moreover, a scenario which is not considered in existing map interaction models is the creation of pinwheels from a pinwheel free OP pattern. Such a scenario is possible if a pinwheel rich state corresponds to the energetic ground state of the model. To lift these ambiguities it would be highly desirable to have a model in which one can identify stationary, pinwheel rich states of the dynamics and also mathematically demonstrate their stability.

\section{Stabilization by long-range horizontal connections}

The second hypothesis for pinwheel stabilization originates from the observed geometry of cortical connectivity. Neurons in the visual cortex form extensive connections horizontal to the cortical surface, linking different orientation columns over distances up to several millimeters [39 48]. In a seminal study Wolf has shown that the inclusion of such long-range horizontal connections into a dynamics of an OP map can lead to the stabilization of pinwheels [49, 50]. Moreover, with increasing spatial extend of these connections the predicted OP layout becomes more irregular. Considering the geometric relationships between different cortical maps we thus ask how the spatial irregularity of OP maps influences the layout of co-evolving maps like that of OD. Several inter-species differences in the cortical map layout have been reported. In particular, the OD layout ranges from a patchy arrangement observed in cat visual cortex to a more stripe-like structure found in monkeys. Can map interactions explain these layout differences? To answer this question an appropriate model should capture species variations in the basic parameters. In the current thesis we therefore investigate how the observed differences in cortical map layout can occur by interactions among maps 
with different typical wavelengths.

\section{Multistability and pattern selection}

One aim of this thesis is to examine the diversity of potential patterns which are offered by models for the development of OP maps. The OP dynamics including long-range interactions leads to a solution class with a huge number of stationary solutions. Remarkably, these solutions share the same stability and energy properties. Moreover, they substantially vary in their quantitative properties such as the pinwheel density. Despite this variation, the ensemble average over the different multistable solutions leads, for a large interaction range, to a pinwheel density that equals the mathematical constant $\pi$ in agreement with experimentally obtained densities. Multistability and energetic degeneracy substantially simplifies the analysis of the OP dynamics which in this case can be restricted to the analysis of a single stationary solution. An important question we want to answer in the current thesis is how robust is this degeneracy and, if it is lifted, how to deal with those numerous solutions. We identify two mechanisms that can lift the described degeneracy and thus lead to pattern selection within this solution class. Multistability and energetic degeneracy originate from an assumed symmetry of the OP dynamics, namely permutation symmetry. Given this symmetry, the degeneracy is guaranteed when pattern formation takes place close to the pattern forming instability, where leading order amplitude equations are sufficient to describe the dynamics. We therefore study pattern formation in the presence and absence of permutation symmetry as well as near and far from the pattern forming instability. Broken permutation symmetry not only leads to a selection of solutions within the highly multistable solution class but also extends the set of potential solution classes. In the current thesis we therefore introduce new classes of solutions to the OP dynamics which not have been considered before and discuss several questions that arise for these solutions: Does a new solution class contain biologically plausible solutions which can become stable? If there are unrealistic solutions, how can their stability be excluded? How sensitive are the new solutions and thus their pinwheel densities to model details?

Outline of the thesis

This thesis is organized as follows. Chapter 2 gives a brief introduction of the biological background. This is followed by Chapter 3 introducing pattern formation models for the development of visual cortical maps. In particular, we consider a general model of interactions between OP maps and another feature map where the inclusion of interaction terms is strictly justified by symmetry considerations. In Chapter 4 we discuss four representative examples of inter-map coupling energies and apply the general model to interactions between OP and OD maps. In particular, we show that inter-map coupling is able to stabilize pinwheel rich OP patterns which would disintegrate into pinwheel free stripe patterns in the uncoupled case. We identify a criterion for the stability of pinwheel rich solutions, namely the existence of a bias in the OD map towards the inputs from one eye. The resulting patterns have regular spatial layouts which we characterize in detail. To achieve an irregular spatial layout we include non-local interaction terms into the dynamics of the OP map in Chapter 5. We 
investigate the influence of the OP map on the layout of the co-evolving OD map. We show that inter-map coupling can transform OD stripe patterns into a spatially irregular layout which resembles the diverse OD layouts such as those of cat and macaque visual cortices. The OP dynamics has a vast number of stable stationary solutions which are energetically degenerate. This degeneracy is valid in the leading order of a perturbative expansion close to the bifurcation point. In Chapter $\mathbf{6}$ we thus study pattern selection far from the bifurcation point. We derive higher order corrections to the amplitude equations and show that the degeneracy is lifted and solutions with a relatively low pinwheel density are energetically selected. We study another mechanism of pattern selection in Chapter 7 We generalize the OP dynamics by breaking the permutation symmetry which leads to pattern selection already at leading order. In addition to the dominant solution class in the permutation symmetric case we identify an additional class which can become dominant in the symmetry broken case, and which is rather insensitive to model details. Finally, we summarize the main results in Chapter 8 and discuss its consequences and potential future directions.

A notation guide is provided on page 165. 


\section{Biological Basics}

To formulate appropriate mathematical models for visual cortical development it is important to understand the basics of the neuronal anatomy and physiology. We thus briefly sketch the main biological facts of the visual system which are essential for this work.

\subsection{From the retina to the cortex}

The visual pathway that conveys visual information from the retina to the visual cortex is sketched in Fig. 2.1. The retina is the first processing stage of visual signals where light, entering through the eye, is transformed into spike patterns. The retinal ganglion cells represent the output of the retina. Their axons leave the retina and travel through the optic nerve to the optic chiasm. Here, a partial crossing of the axons takes place. Each optic nerve branches such that parts of the fibers target the contralateral Lateral Geniculate Nucleus (LGN) at the side opposite to its origin, whereas the rest targets the ipsilateral LGN at the same side as the eye of origin. From that stage on the optic nerves carry information from both eyes. The LGN in the thalamus is the major target of the retinal ganglion cells and relays their information to the primary visual cortex via the optic radiation. This segregation is maintained as the axons enter the cortex. The cells of the LGN then project to their main target, the primary visual cortex (V1) which is located at the occipital lobe of the neocortex. The primary visual cortex, like the other parts of the cortex, is composed of a two-dimensional, slightly folded sheet of neurons and connections between them. V1 of the left hemisphere thus receives input from the right visual field of the ispilateral (left) as well as of the contralateral (right) eye and vice versa. Beyond V1, visual information processing is passed to higher cortical areas, such as the secondary visual cortex V2 or areas MT and IT.

\subsection{Maps in the visual cortex}

In the visual cortex, as in most areas of the cerebral cortex, information is processed in a 2dimensional array of functional modules, called cortical columns [52,53. Individual columns are groups of neurons extending vertically throughout the entire cortical thickness that share many functional properties. In a plane parallel to the cortical surface, neuronal selectivities vary systematically, so that columns of similar functional properties form a systematically organized 2-dimensional pattern, known as functional cortical maps. These patterns exhibit a typical lateral spacing $\Lambda$ which is in the range of 1 millimeter. The visual pathways are organized in a highly topographic manner, neighboring locations in the visual field map onto 


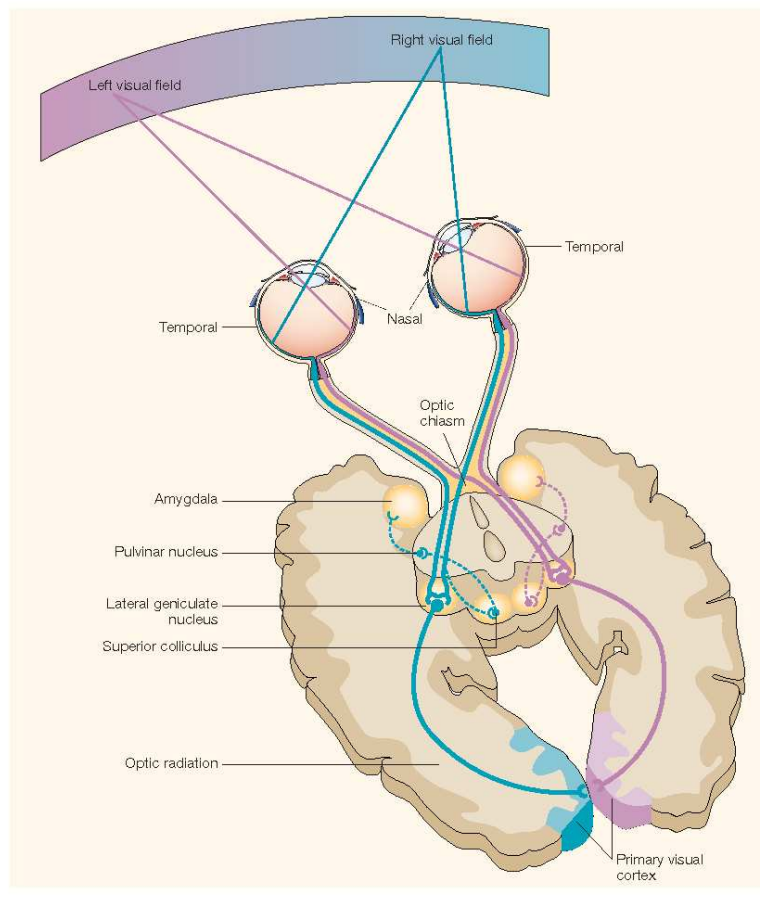

Figure 2.1: A sketch of the human visual pathway. Stimuli on the left side of the field of view map on the temporal retina of the right eye and the nasal retina of the left eye. Visual information from the retina is relayed via a thalamic nucleus to the primary visual cortex at the occipital pole of the brain, adapted from 51.

neighboring locations in the cortex. This feature is called retinotopy and V1 is said to contain a retinotopic map. Superimposed on the retinotopic map are maps of additional stimulus features, as illustrated in Fig. 2.2.

\subsubsection{Ocular dominance maps}

V1 is the first stage in the visual pathway where individual neurons have access to information from corresponding locations in both retinae. Many cells in the visual cortex are selective to whether the input comes from the ipsilateral or the contralateral eye. This ocular dominance (OD) is found to alternate regularly between groups of neurons giving rise to an OD map. Experimentally ocular dominance maps can be visualized using radioactive tracers such as $\left[{ }^{3} \mathrm{H}\right]$-proline or 2-deoxyglucose and also by optical imaging of intrinsic signals. For the proline labeling the injection into one eye via the axonal transport the tracer appears in the primary visual cortex. In this way the tracer is enriched in the cells that are in connection with the injected eye. The typical spatial layout of OD maps for cats and macaque monkeys is depicted in Fig. 2.3. It is known that there is a bias of cortical response toward the contralateral eye at an early stage of postnatal development. This bias has been observed in many different species including ferrets [57], cats [58], monkeys [56], and mice [59]. While in young animals there is a huge over-representation of the contralateral eye, this over-representation is reduced as the animal matures. This bias might have evolutionary reasons. In lower vertebrates, such as fish, that lack binocular vision, there is a total crossing of the afferents leading to a complete contralateral representation. The early visual system of mammals thus might reflect this bias. Ocular dominance maps can be described by a real valued two dimensional field $o(\mathbf{x})$, where 


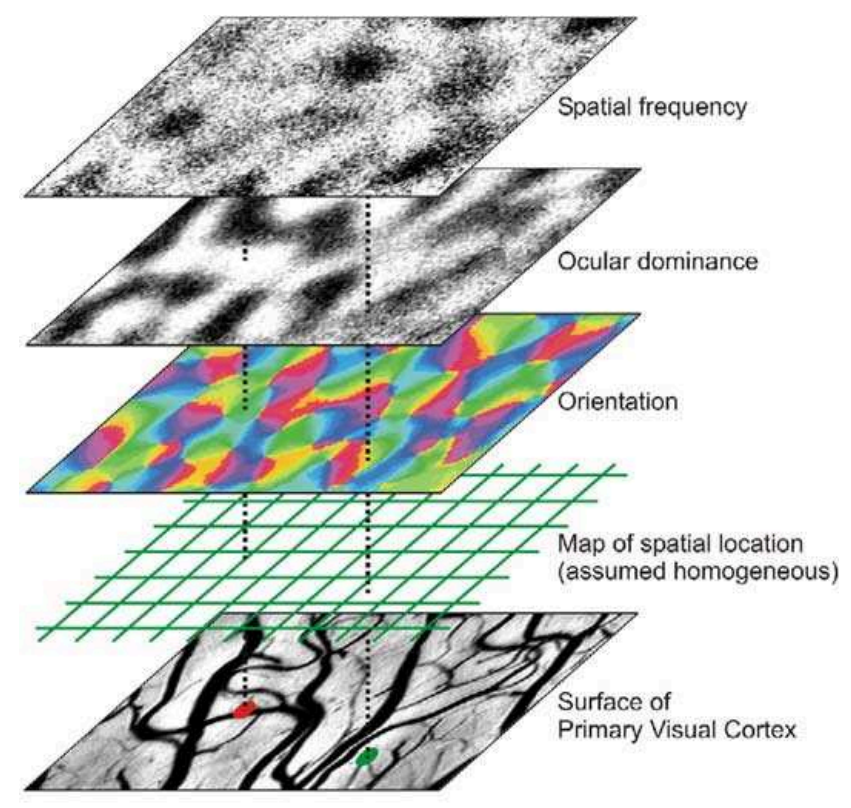

Key:

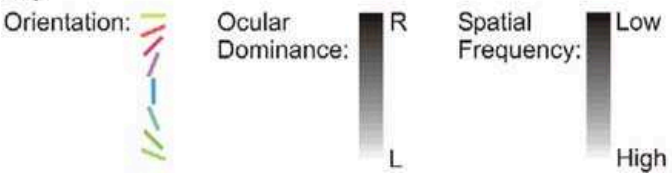

Figure 2.2: Maps in the visual cortex. Schematic of maps of spatial location (retinotopy), orientation preference, ocular dominance, and spatial frequency over a patch of the primary visual cortex. Neurons at the cortical position marked in red would be tuned to the particular stimulus location, orientation, ocular dominance, and spatial frequency identified by the dotted line leading from the red spot. Neurons in the green spot would be tuned to the different set of values indicated by its dotted line. The key gives color codes identifying tunings for the different features (from [54]).

$o(\mathbf{x})<0$ indicates ipsilateral eye dominance and $o(\mathbf{x})>0$ contralateral eye dominance of the neuron located at position $\mathbf{x}$. The magnitude indicates the strength of the eye-dominance.

\subsubsection{Orientation preference maps}

Neurons in the visual cortex are also selective to the orientation of a presented stimulus. Orientation columns in the visual cortex are composed of neurons preferentially responding to visual contours of a particular stimulus orientation. Experimentally, the pattern of orientation preferences can be visualized using optical imaging methods. Optical imaging of intrinsic signals is based on the fact that the optical properties differ in activated vs. less activated parts of cortical tissue. This is utilized to record patterns of activity from light reflectance. In a typical experiment, the activity patterns $E_{k}(\mathbf{x})$ produced by stimulation with a grating of orientation $\vartheta_{k}$ are recorded, see Fig. 2.4(A). Here $\mathbf{x}$ represents the location of a column in the cortex. Using the activity patterns $E_{k}(\mathbf{x})$, a field of complex numbers $z(\mathbf{x})$ can be constructed that completely describes the pattern of orientation columns. This complex field is obtained by vector averaging activity patterns over the different stimuli i.e.

$$
z(\mathbf{x})=\sum_{k} e^{2 \imath \vartheta_{k}} E_{k}(\mathbf{x})
$$


(a)

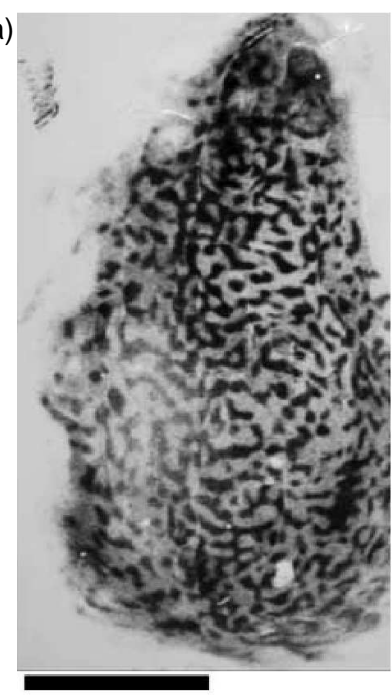

(b)

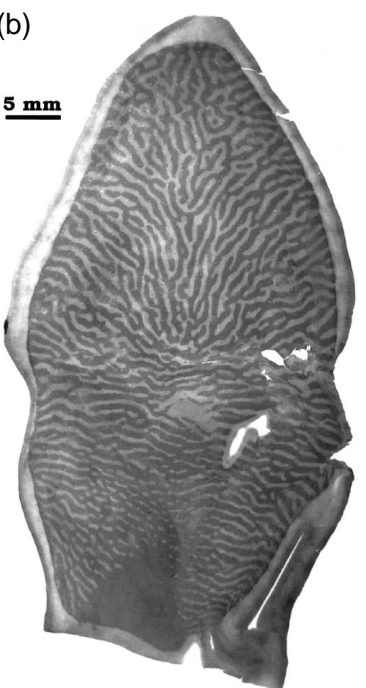

(c)

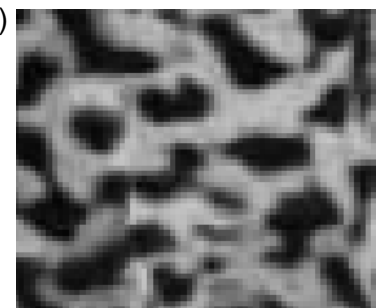

(d)

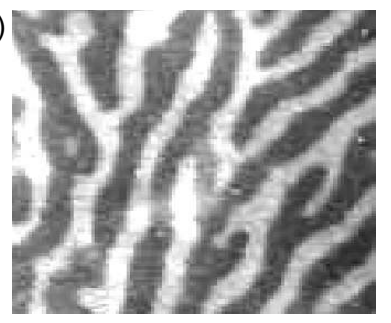

Figure 2.3.: Comparison of the OD pattern for different species. (a) OD pattern in V1 of a cat obtained by $\left[{ }^{3} \mathrm{H}\right]$ proline labeling (scale bar $10 \mathrm{~mm}$ ), from [55], (b) OD pattern in V1 of a macaque monkey obtained by cytochrome oxidase staining, from [56]. (c,d) Display detail of $(\mathbf{a}, \mathbf{b})$.

The pattern of preferred stimulus orientation $\vartheta$ is then obtained by

$$
\vartheta(\mathbf{x})=\frac{1}{2} \arg (z)
$$

The modulus $|z(\mathbf{x})|$ is a measure of the selectivity at cortical location $\mathbf{x}$. Typical examples of activity patterns and the pattern of orientation preference derived from them are shown in Fig. 2.4 (A,B). Orientation preference maps are characterized by so called pinwheels, regions in which columns preferring all possible orientations are organized around a common center in a radial fashion. The centers of pinwheels are point discontinuities of the field $\vartheta(\mathbf{x})$ where the mean orientation preference of nearby columns changes by 90 degrees, see Fig. 2.4 (C,D). Pinwheels can be characterized by a topological charge $q$ which indicates whether the orientation preference increases clockwise or counterclockwise around the pinwheel center,

$$
q_{i}=\frac{1}{2 \pi} \oint_{C_{i}} \nabla \vartheta(\mathbf{x}) d \mathbf{s}
$$

where $C_{i}$ is a closed curve around a single pinwheel center at $\mathbf{x}_{i}$. Since $\vartheta$ is a cyclic variable in the interval $[0, \pi]$ and up to isolated points is a continuous function of $\mathbf{x}, q_{i}$ can only have values

$$
q_{i}=\frac{n}{2}
$$

where $n$ is an integer number [61]. If its absolute value $\left|q_{i}\right|=1 / 2$, each orientation is represented only once in the vicinity of a pinwheel center. In experiments, only pinwheels with a topological charge of $\pm 1 / 2$ are observed, which correspond to simple zeros of the field $z(\mathbf{x})$. The existence of pinwheels has been confirmed in a large number of species and is therefore 

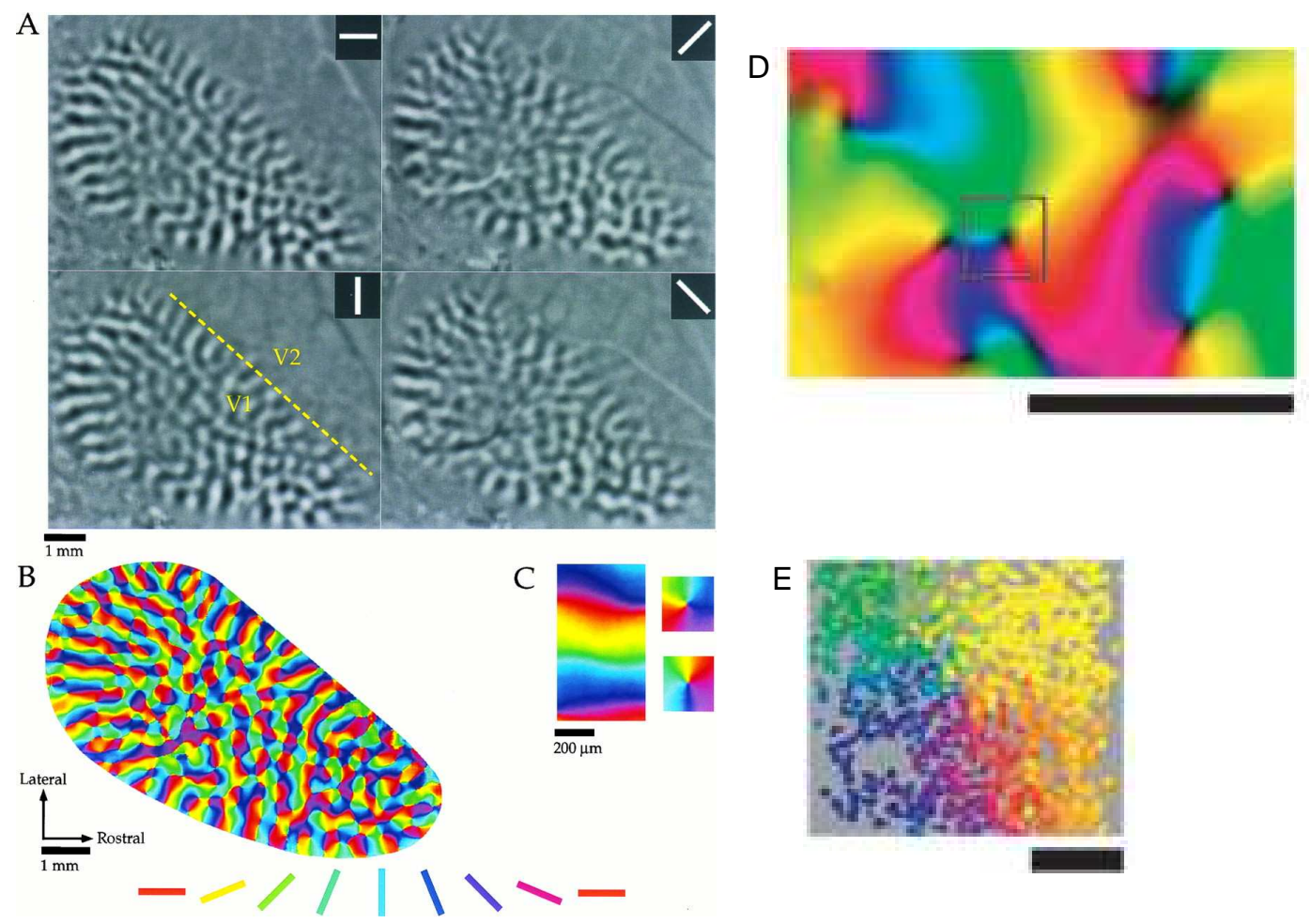

Figure 2.4.: Pattern of orientation columns (A-C) in the primary visual cortex of a tree shrew visualized using optical imaging of intrinsic signals (from [39]). (A) Activity patterns. The bars depict the orientation of the stimulus. Black areas indicate areas of the cortex that were preferentially activated by the given stimulus. (B) Orientation preference map obtained by vector summation of data obtained for each angle. (C) Typical structures that can be found in orientation preference maps such as linear zones and pinwheels. (D) OP map of cat visual cortex. Darker colors represent less selective responses. The square region indicates the typical structure of pinwheels, scale bar $1 \mathrm{~mm}$ (E) Pinwheel with single cell resolution, scale bar $100 \mu \mathrm{m}$, from [60].

believed to be a general feature of orientation preference maps. Recent research has clarified the microscopic structure of pinwheel centers, revealing that radial organization is layed down with single cell precission [60], see Fig. 2.4(E).

Pinwheels in OP maps are abundant, several hundreds of pinwheels are found for instance in the visual cortex of cats. The number of pinwheels per hypercolumn is thus a good quantitative measure to distinguish different OP maps. We define the pinwheel density $\rho$ as the mean number of pinwheels in a region of size $\Lambda^{2}$. Several studies reported a considerable interspecies variability in the pinwheel density [62 64]. Kaschube et al. [16] developed a novel pinwheel analysis method that takes the spatial inhomogeneities in the column spacing $\Lambda$ into account. They found that the pinwheel density was statistically indistinguishable among all three analyzed species ( $\rho=3.12$ tree shrew, $\rho=3.18$ galago, $\rho=3.16$ ferret) and is further statistically indistinguishable from the mathematical constant $\pi$.

The exact set of V1 tuning properties differs for different species. As depicted in Fig. 2.2 in addition to orientation selectivity and ocular dominance neurons in the visual cortex can 


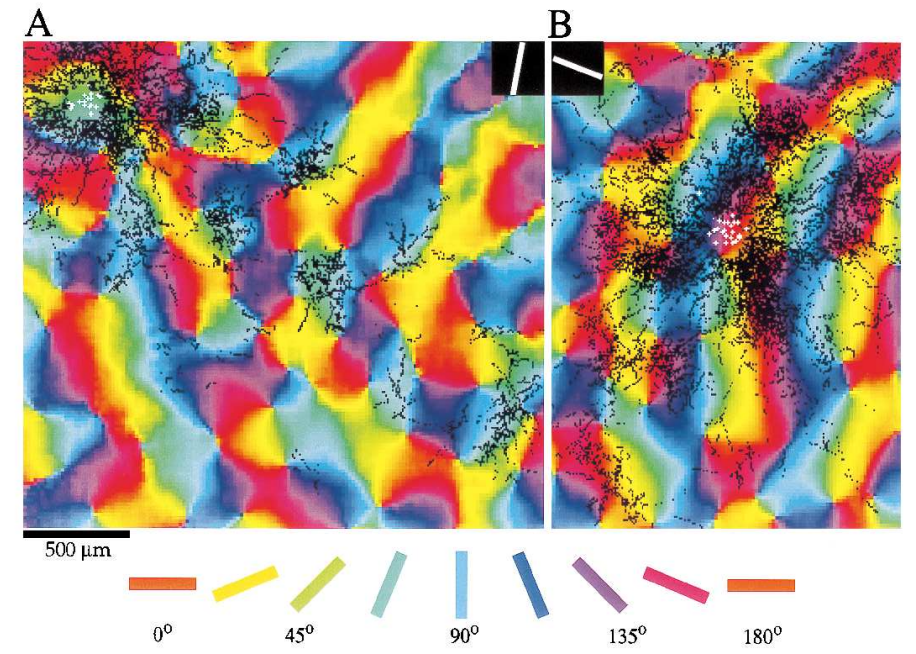

Figure 2.5: Long-range horizontal connections extend over several millimeter parallel to the cortical surface (tree shrew, superimposed on the OP map). White crosses indicate the location of cells that are injected with the tracer biocytin. Black symbols are the labeled axons, (from [39]).

be selective to more stimulus features like the direction of motion [65, 66] or spatial frequency $[33,67,68]$.

\subsection{Long-range connections}

Besides a strong connectivity perpendicular to the cortical surface between neurons from different layers within a column, neurons also form extensive connections horizontal to the cortical surface. These connections extend over several millimeters parallel to the cortical surface and are therefore called long-range horizontal connections. These connections allow the cells to integrate visual information from outside their receptive fields. Figure 2.5 shows the long-range connections found in tree shrew. The typical distance of long-range connections is similar to the wavelength of the columnar patterns giving rise to the idea that these connections are correlated with orientation selectivity. Indeed, the connections are clustered and preferentially connect domains of similar orientation preference. This has been demonstrated for different species in a variety of experiments [39 48. The relations between long-range connections and other visual cortical maps is less clear. In the primary visual cortex of cats, long-range intrinsic connections extend between domains of left and right eye dominance with equal probability [48, 69, 70], whereas in macaque monkeys intrinsic long-range connections seem to have a preference for the same-eye target [71,72].

\subsection{Geometric relationships}

The different visual cortical maps coexist in the same piece of cortex. It is thus natural to expect that there might exist relations between different maps. Indeed, the different cortical maps are not independent of each other but exhibit definite geometric relationships. Experimentally this has been shown in a variety of species [68, 73, 75]. These experiments show that iso-orientation lines intersect OD borders preferentially at right angles, see Fig. 2.6. 
A

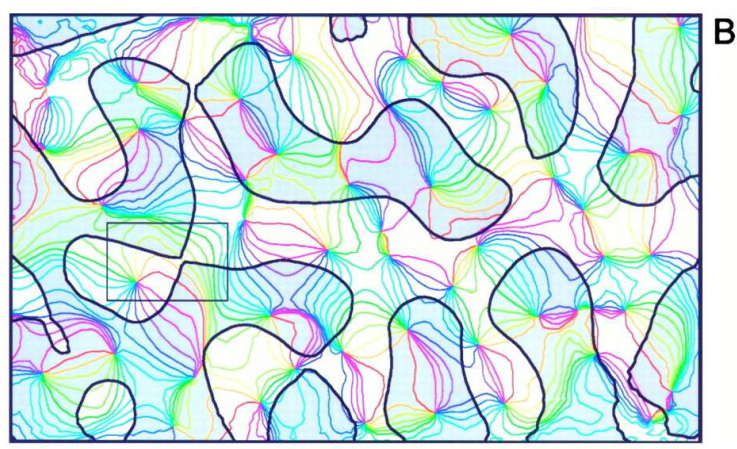

B

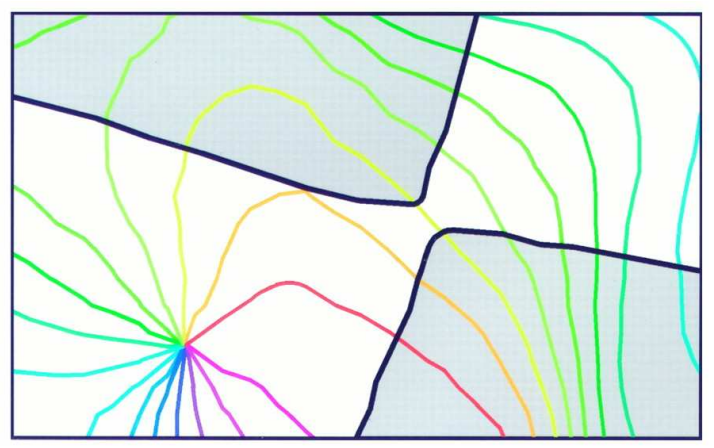

Figure 2.6.: (A) Geometric relationships between OD and OP maps in cat visual cortex. Colored lines: Iso-orientation lines, black lines: OD borders. Gray denotes contralateral eye dominance. (B) Enlarged detail from (A), (from [68]).

This tendency might be species dependent and more pronounced in primates than in cats and less pronounced in ferrets. Further, it has been shown that there is a clear tendency for the pinwheel centers to be at the OD extrema. Intuitively, these findings are consistent with the concept of uniform coverage [23]. Uniform coverage means that rapid changes in one feature component are correlated with slow changes in the other components. Orthogonal relationships between columns thus have the advantage of allowing many combinations of stimulus properties to be represented in a small cortical region. Apart from OP and OD maps, such correlations have been found also in other visual cortical maps such as spatial frequency maps [68]. This suggests that the geometric correlations reflect an universal organization principle in the visual cortex. The presence of mutual dependence among multiple maps thus could provide an important clue for understanding the development of visual information representations.

\subsection{Variation across different species}

The existence of a specific columnar system is species dependent. Rodents have orientation selective cells but lack OP maps [76]. Mice, rats and tree shrews also lack OD maps [77. Even an inter-individual variability of the formation of OD columns has been observed [78]. The spatial layout of OP and OD maps, when observed, varies significantly between different species. For instance the spatial layout of OD maps differs between cats and macaque monkeys, see Fig. 2.3. For cats the spatial organization of OD domains shows an isotropic patchy structure. In the case of macaque monkeys the OD layout shows parallel bands with a clear tendency of elongation perpendicular to the area boundary.

A second interspecies difference concerns the average wavelength $\Lambda$ of the different columnar systems. In cats, the average wavelength of iso-orientation domains is approximately equal to that of OD columns $\Lambda_{O P} \approx \Lambda_{O D}$, [55,64,79]. This relation is different for example in macaque monkeys, where the average wavelength of iso-orientation domains is smaller than that of OD columns, $\Lambda_{O P} \approx 0.83 \Lambda_{O D}$ [56,75]. But note that there is also a substantial variation across different animals for a given species concerning the map layout and even whether there is 
a map at all [78. Species differences could also arise from the shape of the primary visual cortex, which is more elongated in the macaque monkey than in the cat [80]. An appropriate model for the development of cortical maps should be capable of explaining such inter-species differences.

\subsection{Development of cortical maps}

The structure of the visual cortex is set up very early in life. In normal development orientation columns first form at about the time of eye opening which is at about post natal day (PD) 10 in cats and PD 31 in ferrets [57,81,82. What is guiding the development of cortical maps? While many experiments suggest they are determined by neural activity, some indicate that molecular cues might also be involved [83]. There are indications that an initial map can be established without any visual information. For instance experiments in which animals are reared in darkness show OP maps [42]. The large number of neurons and synapses in the cortex, however, makes it unlikely to completely specify neural connectivity by genetic information. Instead, there are numerous indications that the initial map is maintained and refined through visual information. Evidence from this hypothesis comes from experiments manipulating the sensory input to the cortex. Deprivation of visual input for instance by lid-suture can cause the loss of orientation selectivity [42. Kittens reared in a striped environment show twice as much surface area to the experienced orientation as to the orthogonal one [84]. Monocular deprivation leads to substantial changes in the OD map, for instance inducing a bias of visual response the intact eye [85]. A striking example of self-organization are experiments in which tadpoles are implanted a third eye which leads to a segregation of alternating eye specific domains [86]. In another remarkable experiment supporting this hypothesis the visual input was rewired to the auditory cortex in ferrets [87]. Projections from the retina were experimentally redirected to the auditory pathway at a very early stage of development. As a consequence, patterns of orientation preference have been recorded in the area that normally would form the primary auditory cortex.

To conclude, after an initial phase in which the cortical circuitry is established follows a critical period of refinement through detailed sensory input. This period lasts for about three month for instance in cats. Although during the critical period the cortex is most susceptible to changes in the input, there are indications that map plasticity is maintained also in the adult visual cortex [88].

Based on the current experimental data there is no statement on whether the development of one map precedes the other. In ferrets optical imaging studies suggest that OP develops slightly before OD [89. In cats and macaques, however, experimental data have not definitively established the precise ordering of OP and OD map development. In monkeys the OD map emerges before birth [90] and the OP map is present very soon after birth [91,92, though it may emerge earlier. In cats, although it was originally thought that the OD map emerges starting at about 3 weeks after birth [93], more recent optical imaging data suggests that both OP and OD maps are present as early as 2 weeks after birth [58]. 


\section{Model Basics}

In this chapter we introduce dynamical models for visual cortical development and describe the basic concepts to deal with such models. The description of the development of visual cortical maps on the level of individual neurons and synapses is very complex due to the large number of degrees of freedom. Moreover, techniques such as dimension reduction models are mathematically hardly tractable. Instead we describe this development by the time evolution of order parameter fields. We consider a field model of an OP map, described by the field $z(\mathbf{x}, t)$, and another feature map $o(\mathbf{x}, t)$ which is of the form

$$
\begin{aligned}
& \partial_{t} z(\mathbf{x}, t)=F[z(\mathbf{x}, t), o(\mathbf{x}, t)] \\
& \partial_{t} o(\mathbf{x}, t)=G[z(\mathbf{x}, t), o(\mathbf{x}, t)],
\end{aligned}
$$

where $F[z, o]$ and $G[z, o]$ are nonlinear operators. Although the field $o(\mathbf{x}, t)$ is mainly considered as the field of OD it can represent several other feature maps such as the map of spatial frequency. Various biologically detailed models have been cast to this form [18,22, 94]. The convergence of this dynamics towards an attractor is assumed to represent the process of maturation and optimization of the cortical circuitry. Therefore we assume a variational dynamics $F[z, o]=-\frac{\delta E}{\delta \bar{z}}, G[z, o]=-\frac{\delta E}{\delta o}$. The system then relaxes towards the minima of the energy $E$. In the following sections we specify this energy functional by symmetries and qualitative properties of the underlying system.

\subsection{A generalized Swift-Hohenberg model}

Viewed from a dynamical systems perspective, the activity-dependent remodeling of the cortical network described in Section 2.6 is a process of dynamical pattern formation. Visual cortical maps emerge from an almost unselective homogeneous state. This scenario can be described in terms of a supercritical bifurcation where the homogeneous state loses its stability and spatial modulations of some typical wavelength $\Lambda$ start to grow. A well studied model reproducing this behavior is of the Swift-Hohenberg type [4, 95]. Many other pattern forming systems occurring in different physical, chemical, and biological contexts (see for instance $[2,3,6,13,14])$ have been cast into a dynamics of this type. Its dynamics in case of the OP map is of the form

$$
\partial_{t} z(\mathbf{x}, t)=\hat{L} z(\mathbf{x}, t)-N[z(\mathbf{x}, t)]
$$


with the linear Swift-Hohenberg operator

$$
\hat{L}=r-\left(k_{c}^{2}+\Delta\right)^{2},
$$

$k_{c}=2 \pi / \Lambda$, and $N[z(\mathbf{x}, t)]$ a nonlinear operator. A nonlinearity we thoroughly analyze in this thesis is given by

$$
\begin{aligned}
N[z, z, \bar{z}]= & (g-1)|z(x)|^{2} z(x)+ \\
& \frac{2-g}{2 \pi \sigma^{2}} \int d^{2} y e^{-|x-y|^{2} / 2 \sigma^{2}}\left(z(x)|z(y)|^{2}+\frac{1}{2} \bar{z}(x) z(y)^{2}\right) .
\end{aligned}
$$

The parameter $g \in[0,2]$ balances the influence of local or non-local interactions, while $\sigma \geq 0$ is the width of the non-local interactions. For $1<g \leq 2$ both local and non-local interaction have a negative sign and thus stabilize the dynamics, whereas for $0 \leq g \leq 1$ only the non-local part stabilizes the dynamics.

In Fourier representation $\hat{L}$ is diagonal with the spectrum

$$
\lambda(k)=r-\left(k_{c}^{2}-k^{2}\right)^{2} .
$$

The spectrum exhibits a maximum at $k=k_{c}$, see Fig. 3.1(a). For $r<0$ all modes are damped since $\lambda(k)<0, \forall k$ and only the homogeneous state $z(\mathbf{x})=0$ is stable. This is no longer the case for $r>0$ when modes on the critical circle $k=k_{c}$ acquire a positive growth rate and now start to grow, resulting in patterns with a typical wavelength $\Lambda=2 \pi / k_{c}$.

While the linear part of the dynamics establishes a typical wavelength, the nonlinearity in the dynamics leads to the selection of the final pattern. Snapshots of the time evolution of Eq. (3.2) with the nonlinearity Eq. (3.4) are shown in Fig. 3.1(c). The dynamics is initialized with a random OP map with low selectivity. The time is given in units of the intrinsic timescale $T=r t$ which is associated with the growth rate of Eq. (3.3). Several different stages of the dynamics can be identified. The linear part forces modes on the critical circle to grow with rate $r$ while strongly suppressing modes off the critical circle, see Fig. 3.1(a). The OP map becomes more and more ordered in this linear phase as a single wavelength is selected. The total power of the field is given by

$$
P(t)=\left\langle|z(\mathbf{x}, t)|^{2}\right\rangle_{\mathbf{x}}
$$

where \langle\rangle$_{\mathbf{x}}$ denotes the spatial average. The power reflects the different growth rates among modes. The time evolution of the power is depicted in Fig. 3.1(b). Initially the power decreases slightly due to the suppression of modes outside the circle of positive growth rate. At $T \approx 1$ there is a rapid increase and then a saturation of the power. The amplitudes of the Fourier modes reach their stationary values and $P \propto r$. At this stage of the evolution the influence of the nonlinear part is comparable to that of the linear part. Once the modes saturated the phase of nonlinear competition between the active modes along with a reorganization of the structure of the OP map starts. The competition between active modes leads 
(a)
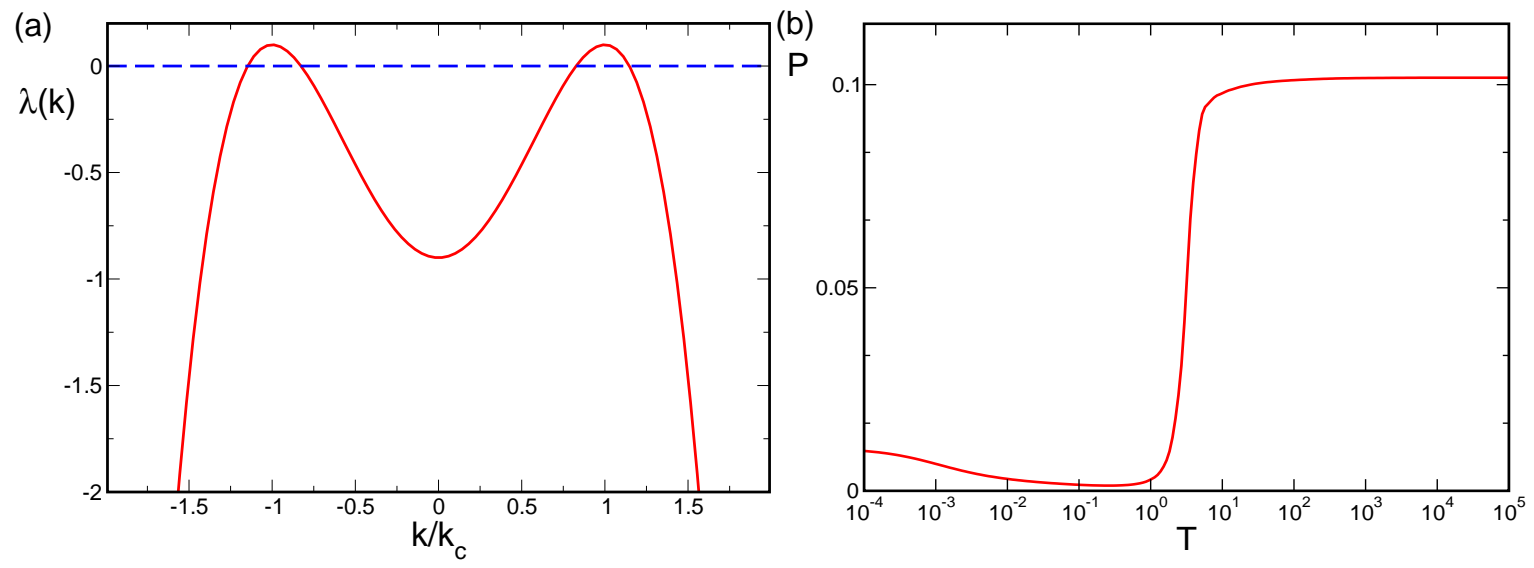

(c)
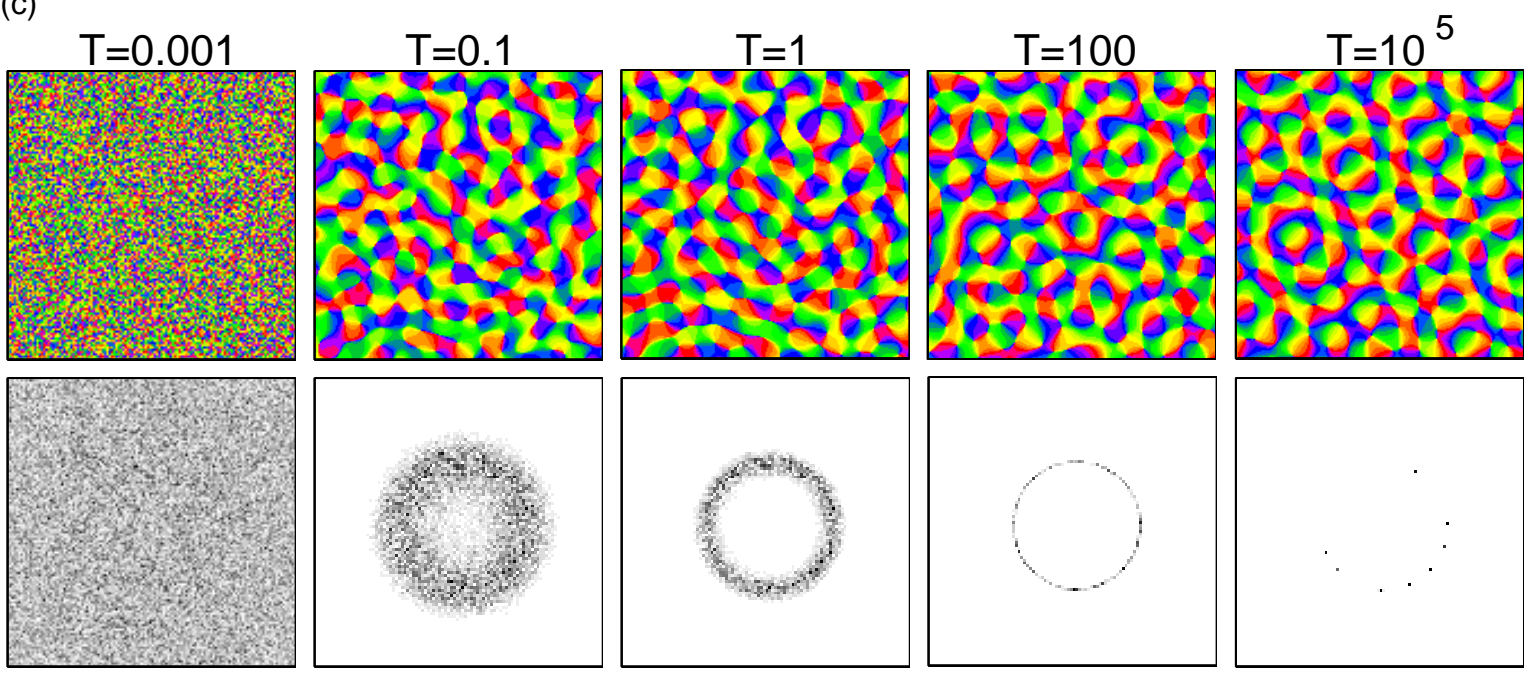

Figure 3.1.: Swift-Hohenberg equation (a) Cross section through the spectrum $\lambda(k)$ of the Swift-Hohenberg operator Eq. (3.5), $r=0.1$. (b) Time evolution of the Power Eq. (3.6). (c) Snapshots of the time evolution of Eq. (3.2). Top panel: orientation preference, bottom panel: power spectrum. Parameters: $r=0.1, g=0.98, \sigma=1.4 \Lambda, \Gamma=22,128 \times 128$ mesh.

to pattern selection. The final pattern then consists of distinct modes in Fourier space. Once the active modes are selected, a relaxation of their phases takes place. This will be discussed in detail in Section 6.4.

Inter-map coupling can influence the time evolution on all stages of the development depending on whether this coupling affects only the nonlinear part or also the linear one. When incorporating additional maps into the system we will in all cases rescale the dynamics by the bifurcation parameter of the OP map i.e. $T=r_{z} t$.

\subsection{Inter-map coupling: A symmetry-based analysis}

The pattern formation model described above is now extended by including the dynamics of the real OD field $o(\mathbf{x}, t)$. The coupled Swift-Hohenberg equations are assumed to have the 
form given by

$$
\begin{aligned}
& \partial_{t} z(\mathbf{x}, t)=\hat{L}_{z} z(\mathbf{x}, t)-N[z(\mathbf{x}, t)]-\frac{\delta U}{\delta \bar{z}} \\
& \partial_{t} o(\mathbf{x}, t)=\hat{L}_{o} o(\mathbf{x}, t)-\widetilde{N}[o(\mathbf{x}, t)]-\frac{\delta U}{\delta o}+\gamma,
\end{aligned}
$$

where $\hat{L}_{\{o, z\}}=r_{\{o, z\}}-\left(k_{c,\{o, z\}}^{2}+\Delta\right)^{2}$ and $\gamma$ a constant. To account for the species differences in the wavelengths of the pattern we choose two typical wavelengths $\Lambda_{z}=2 \pi / k_{c, z}$ and $\Lambda_{o}=$ $2 \pi / k_{c, o}$. The dynamics of $z(\mathbf{x}, t)$ and $o(\mathbf{x}, t)$ is coupled by interaction terms which can be derived from a coupling energy $U$.

As we use a phenomenological approach the inclusion and exclusion of various terms has to be strictly justified. We do this by symmetry considerations. The constant $\gamma$ breaks the inversion symmetry $o(\mathbf{x})=-o(\mathbf{x})$ of inputs from the ipsilateral $(o(\mathbf{x})<0)$ or contralateral $(o(\mathbf{x})>0)$ eye. The inter-map coupling energy $U$ is assumed to be invariant under this inversion. The primary visual cortex shows no anatomical indication that there are any prominent regions or directions parallel to the cortical layer [12. Besides invariance under translations $\hat{T}_{y} z(\mathbf{x})=z(\mathbf{x}-\mathbf{y})$ and rotations $\hat{R}_{\phi} z(\mathbf{x})=z\left(R_{\phi}^{-1} \mathbf{x}\right)$ of both maps we require that the dynamics should be invariant under orientation shifts $z(\mathbf{x}) \rightarrow e^{\imath \vartheta} z(\mathbf{x})$. Note, however, that the proposed set of symmetries has been questioned raising the possibility that the orientation shift symmetry is not a proper symmetry of OP map development [96]. A general coupling energy term can be expressed by integral operators which can be written as a Volterra series

$$
E=\sum_{u=u_{o}+u_{z}} \int \prod_{i=1}^{u_{o}} d^{2} x_{i} o\left(\mathbf{x}_{i}\right) \prod_{j=u_{o}+1}^{u_{o}+u_{z} / 2} d^{2} x_{j} z\left(\mathbf{x}_{j}\right) \prod_{k=u_{o}+u_{z} / 2+1}^{u} d^{2} x_{k} \bar{z}\left(\mathbf{x}_{k}\right) K\left(\mathbf{x}_{1}, \ldots, \mathbf{x}_{u}\right)
$$

with an $u$-th. order integral kernel $K$. Inversion symmetry and orientation shift symmetry require $u_{o}$ to be even and that the number of fields $z$ equals the number of fields $\bar{z}$. The lowest order term, mediating an interaction between the fields $o$ and $z$ is therefore given by $u=4, u_{o}=2$ i.e.

$$
E_{4}=\int d^{2} x_{1} d^{2} x_{2} d^{2} x_{3} d^{2} x_{4} o\left(\mathbf{x}_{1}\right) o\left(\mathbf{x}_{2}\right) z\left(\mathbf{x}_{3}\right) \bar{z}\left(\mathbf{x}_{4}\right) K\left(\mathbf{x}_{1}, \mathbf{x}_{2}, \mathbf{x}_{3}, \mathbf{x}_{4}\right)
$$

Next, we rewrite Eq. (3.9) as an integral over an energy density $U$. We use the invariance under translations to introduce the new coordinates

$$
\begin{aligned}
\mathbf{x}_{m} & =(1 / 4) \sum_{j}^{4} \mathbf{x}_{i} \\
\mathbf{y}_{1} & =\mathbf{x}_{1}-\mathbf{x}_{m} \\
\mathbf{y}_{2} & =\mathbf{x}_{2}-\mathbf{x}_{m} \\
\mathbf{y}_{3} & =\mathbf{x}_{3}-\mathbf{x}_{m} .
\end{aligned}
$$


This leads to

$$
\begin{aligned}
E_{4}= & \int d^{2} x_{m} \int d^{2} y_{1} d^{2} y_{2} d^{2} y_{3} o\left(\mathbf{y}_{1}+\mathbf{x}_{m}\right) o\left(\mathbf{y}_{2}+\mathbf{x}_{m}\right) z\left(\mathbf{y}_{3}+\mathbf{x}_{m}\right) \\
& \bar{z}\left(\mathbf{x}_{m}-\sum_{i}^{3}\left(\mathbf{y}_{i}-\mathbf{x}_{m}\right)\right) K\left(\mathbf{y}_{1}, \mathbf{y}_{2}, \mathbf{y}_{3}\right) \\
= & \int d^{2} x_{m} U_{4}\left(\mathbf{x}_{m}\right) .
\end{aligned}
$$

The kernel $K$ can contain local and non-local contributions. Throughout this thesis the map interactions we discuss are assumed to be local. Non-local terms are discussed in the case of the uncoupled OP dynamics in Chapter 5-7. For local interactions the integral kernel is independent of the locations $\mathbf{y}_{i}$. We expand both fields in a Taylor series around $\mathbf{x}_{m}$

$$
z\left(\mathbf{x}_{m}+\mathbf{y}_{i}\right)=z\left(\mathbf{x}_{m}\right)+\nabla z\left(\mathbf{x}_{m}\right) \mathbf{y}_{i}+\ldots, \quad o\left(\mathbf{x}_{m}+\mathbf{y}_{i}\right)=o\left(\mathbf{x}_{m}\right)+\nabla o\left(\mathbf{x}_{m}\right) \mathbf{y}_{i}+\ldots
$$

For a local energy density we can truncate this expansion at the first order in the derivatives. The energy density can thus be written

$$
\begin{aligned}
U_{4}\left(\mathbf{x}_{m}\right)= & \int d^{2} y_{1} d^{2} y_{2} d^{2} y_{3}\left(o\left(\mathbf{x}_{m}\right)+\nabla o\left(\mathbf{x}_{m}\right) \mathbf{y}_{1}\right)\left(o\left(\mathbf{x}_{m}\right)+\nabla o\left(\mathbf{x}_{m}\right) \mathbf{y}_{2}\right) \\
& \left(z\left(\mathbf{x}_{m}\right)+\nabla z\left(\mathbf{x}_{m}\right) \mathbf{y}_{3}\right)\left(\bar{z}\left(\mathbf{x}_{m}\right)-\nabla \bar{z}\left(\mathbf{x}_{m}\right) \sum_{i}\left(\mathbf{y}_{i}-\mathbf{x}_{m}\right)\right) K\left(\mathbf{y}_{1}, \mathbf{y}_{2}, \mathbf{y}_{3}\right) .
\end{aligned}
$$

Due to rotation symmetry this energy density should be invariant under a simultaneous rotation of both fields. From all possible combinations of Eq. (3.13) only those are invariant in which the gradients of the fields appear as scalar products. The energy density can thus be written as

$$
\begin{aligned}
U_{4} & =f\left(c_{1}, c_{2}, \ldots, c_{8}\right) \\
& =f\left(o^{2}, z^{2}, z \bar{z}, o z, \nabla o \nabla o, \nabla z \nabla z, \nabla z \nabla \bar{z}, \nabla o \nabla z\right),
\end{aligned}
$$

where we suppressed the argument $\mathbf{x}_{m}$. All combinations $c_{j}$ can also enter via their complex conjugate. The general expression for $U_{4}$ is therefore

$$
U_{4}=\sum_{i>j} l_{i j}^{(1)} c_{i} c_{j}+\sum_{i>j} l_{i j}^{(2)} \bar{c}_{i} \bar{c}_{j}+\sum_{i, j} l_{i j}^{(3)} c_{i} \bar{c}_{j}
$$


From all possible combinations we select those which are invariant under orientation shifts and eye inversions. This leads to

$$
\begin{aligned}
U_{4}= & l_{1} o^{4}+l_{2}|z|^{4}+l_{3}(\nabla o \nabla o)^{2}+l_{4}|\nabla z \nabla z|^{2} \\
& +l_{5}(\nabla z \nabla \bar{z})^{2}+l_{6}(\nabla o \nabla o) o^{2}+l_{7}(\nabla z \nabla \bar{z})|z|^{2} \\
& +l_{8}(\nabla z \nabla z) \bar{z}^{2}+l_{9}(\nabla \bar{z} \nabla \bar{z}) z^{2} \\
& +l_{10}(\nabla o \nabla z) o \bar{z}+l_{11}(\nabla o \nabla \bar{z}) o z \\
& +l_{12} o^{2}|z|^{2}+l_{13}|\nabla o \nabla z|^{2}+l_{14}(\nabla z \nabla \bar{z}) o^{2} \\
& +l_{15}(\nabla o \nabla o)|z|^{2}+l_{16}(\nabla z \nabla \bar{z})(\nabla o \nabla o) .
\end{aligned}
$$

The energy densities with prefactor $l_{1}$ to $l_{9}$ do not mediate a coupling between OD and OP fields and can be absorbed into $N[z]$ and $\widetilde{N}[o]$. The densities with prefactors $l_{8}$ and $l_{9}$ (also with $l_{10}$ and $\left.l_{11}\right)$ are complex and can occur only together with $l_{8}=l_{9}\left(l_{10}=l_{11}\right)$ to be real. But these energy densities are not bounded from below as their real and imaginary parts can have arbitrary positive and negative values. The lowest order terms which are real and positive definite are thus given by

$$
U_{4}=l_{12} o^{2}|z|^{2}+l_{13}|\nabla o \nabla z|^{2}+l_{14} o^{2} \nabla z \nabla \bar{z}+l_{15} \nabla o \nabla o|z|^{2}+l_{16}(\nabla z \nabla \bar{z})(\nabla o \nabla o) .
$$

In the following we will discuss how these coupling energies relates to the geometric relationships between OP and OD maps. In experimentally obtained maps iso-orientation lines show the tendency to intersect the OD borders perpendicularly but only the second term in Eq. (3.17) is expected to be sensitive to these intersection angles. In order to be sensitive to intersection angles the energy is expected to contain terms of the form $|\nabla o \nabla \vartheta|$. When we decompose the complex field $z(\mathbf{x})$ into the selectivity $|z|$ and the preferred orientation $\vartheta$ we get

$$
U=l_{13}|\nabla z \nabla o|^{2}=l_{13}|z|^{2}\left(|\nabla o \nabla \ln | z||^{2}+4|\nabla o \nabla \vartheta|^{2}\right) .
$$

If the orientation selectivity is locally homogeneous, i.e. $\nabla \ln |z| \approx 0$, then the energy is minimized if the direction of the iso-orientation lines $(\nabla \vartheta)$ is perpendicular to the OD borders. Moreover, this energy term couples the OD pattern with the position of pinwheels. To see this we decompose the field $z$ into its real and imaginary part

$$
U=l_{13}\left(|\nabla o \nabla \operatorname{Re} z|^{2}+|\nabla o \nabla \operatorname{Im} z|^{2}\right) .
$$

At pinwheel centers the zero contours of $\operatorname{Re} z$ and $\operatorname{Im} z$ cross. Since there $\nabla \operatorname{Re} z$ and $\nabla \operatorname{Im} z$ are almost constant and not parallel the energy can be minimized only if $|\nabla o|$ is small at the pinwheel centers, i.e. the extrema or saddle-points of $o(\mathbf{x})$.

The first term in Eq. (3.17),

$$
U=l_{12} o^{2}|z|^{2}
$$


is also expected to favor pinwheels at OD extrema. Pinwheels are zeros of $z$ and are thus expected to reduce this energy term. They will reduce energy the most when $|o|$ is maximal which should repel pinwheels from OD borders, where $o(\mathbf{x})$ is zero. So the expectation that terms such as Eq. (3.18) and Eq. (3.20) and its higher order variants lead to a repulsion of pinwheels from OD borders has some intuitive appeal. The third and the fourth term in Eq. (3.17) are expected to lead to the opposite behavior. Near pinwheels where $\nabla z$ is constant the energy is minimized if $o$ is zero i.e. at the OD borders. The last term in Eq. (3.17) is expected to behave similar as the first one. At the pinwheel centers the energy is minimized if $\nabla o$ is small i.e. at OD extrema. The next higher order energy terms are given by

$$
U_{6}=o^{2}|z|^{4}+|z|^{2} o^{4}+o^{4} \nabla z \nabla \bar{z}+\ldots
$$

Here the fields $o(\mathbf{x})$ and $z(\mathbf{x})$ enter with an unequal power. In the corresponding field equations these interaction terms enter either in the linear part or in the cubic nonlinearity. We will see in Section 4.4 and 5.4 that interaction terms that enter in the linear part of the dynamics can lead to a suppression of the pattern and possibly to an instability of the pattern solution. Therefore we consider also higher order interaction terms.

These higher order terms contain combinations of terms in Eq. (3.17) and are given by

$$
\begin{aligned}
U_{8}= & o^{4}|z|^{4}+|\nabla o \nabla z|^{4}+o^{4}(\nabla z \nabla \bar{z})^{2}+(\nabla o \nabla o)^{2}|z|^{4} \\
& +(\nabla z \nabla \bar{z})^{2}(\nabla o \nabla o)^{2}+o^{2}|z|^{2}|\nabla o \nabla z|^{2}+\ldots
\end{aligned}
$$

In general, all coupling energies in $U_{4}, U_{6}$, and $U_{8}$ can occur in the dynamics and we restrict to those energies which are expected to reproduce the observed geometric relationships between OP and OD maps. It is important to note that with this restriction we do not miss any essential parts of the model. When using weakly nonlinear analysis the general form of the near threshold dynamics is insensitive to the used type of coupling energy and we therefore expect similar results also for the remaining coupling energies. In this thesis we discuss the four representative examples of coupling energies

$$
U=\alpha o^{2}|z|^{2}+\beta|\nabla z \nabla o|^{2}+\tau o^{4}|z|^{4}+\epsilon|\nabla z \nabla o|^{4}
$$

For this choice of energy the corresponding interaction terms are given by

$$
\begin{aligned}
-\frac{\delta U}{\delta \bar{z}} & =N_{\alpha}[o, o, z]+N_{\beta}[o, o, z]+N_{\epsilon}[o, o, o, o, z, z, \bar{z}]+N_{\tau}[o, o, o, o, z, z, \bar{z}] \\
& =-\alpha o^{2} z+\beta \nabla(a \nabla o)+\epsilon 2 \nabla\left(|a|^{2} a \nabla o\right)-2 \tau o^{4}|z|^{2} z \\
-\frac{\delta U}{\delta o} & =\widetilde{N}_{\alpha}[o, z, \bar{z}]+\widetilde{N}_{\beta}[o, z, \bar{z}]+\widetilde{N}_{\epsilon}[o, o, o, z, z, \bar{z}, \bar{z}]+\widetilde{N}_{\tau}[o, o, o, z, z, \bar{z}, \bar{z}] \\
& =-\alpha o|z|^{2}+\beta \nabla(\bar{a} \nabla z)+\epsilon 2 \nabla\left(|a|^{2} \bar{a} \nabla z\right)-2 \tau o^{3}|z|^{4}+c . c .
\end{aligned}
$$

with $a=\nabla z \nabla o$ and c.c. denotes the complex conjugate. 


\subsection{Analytical and numerical methods}

The equations 3.7 are nonlinear partial differential equations (or even integro-differential equations) and thus finding analytical solutions is in general impossible. We therefore apply a perturbation method called weakly nonlinear analysis. The system exhibits a Turing instability where the unselective state $z(\mathbf{x})=0$ becomes unstable for $r>0$ and modes on the critical circle start to grow. Close to this bifurcation point the field $|z(\mathbf{x})|$ is still small and thus the nonlinearities in Eq. (3.7) are weak. The near-threshold dynamics is governed by so called amplitude equations. Here, the dimensionality of the system is reduced by distinguishing between dynamically active modes, which are growing or neutrally stable, and passive mode, which decay rapidly. The decomposition allows the near-threshold dynamics to be determined purely in terms of the active modes. The passive modes are found to be entirely dependent or 'slaved' on the active modes. Amplitude equations can be determined perturbatively, using the distance above threshold $r$ as a small parameter in a multiple scale analysis. The derivation of amplitude equations is detailed in Sections 4.2 .2 and 4.3 , In the theory of pattern formation, systems close to threshold or far from threshold are known to behave quite differently [4,97]. The emergence of cortical structures may take place substantially far from criticality. For this reason, and to explore the limits of the perturbation expansion, numerical simulations using relatively large values of the bifurcation parameter are important. All results obtained from weakly nonlinear analysis presented in this thesis are confirmed by solving the full field dynamics numerically. We use a fully implicit integrator based on the Crank-Nicolson scheme and a Newton-Krylov solver. To converse the translation invariance of the patterns we use periodic boundary conditions on a mesh ranging from $128 \times 128$ up to $512 \times 512$ pixels. The aspect ratio $\Gamma=L / \Lambda$, with $L$ the system size, ranges from $\Gamma=17$ up to $\Gamma=100$. A brief description of the numerical integration scheme is given in Appendix A.1.

\subsection{Tracking and counting pinwheels}

During the evolution of OD and OP maps we monitor the states from the initial time $T=0$ to the final time $T=T_{f}$ using about 150 time frames. To account for the various temporal scales the dynamics encounters, the time frames are separated by exponentially increasing time intervals. Pinwheel centers are identified as the crossing of the zero contour lines of the real and imaginary parts of $z(\mathbf{x})$. During time evolution we track all the pinwheel positions and, as the pinwheels carry a topological charge, we divide the pinwheels according to their charge. The distribution of pinwheel distances indicates the regularity and periodicity of the maps. Therefore, we calculate the minimal distance between pinwheels, measured in units of the column spacing $\Lambda$ during time evolution. In simulations we use periodic boundary conditions. We account for a 'wrap around' effect by searching also for corresponding pinwheels in periodically continued maps.

The rearrangement of OP maps leads to annihilation and creation of pinwheels in pairs. Be- 
tween two time frames at $T_{i}$ and $T_{i+1}$ we identified a pinwheel to move if two pinwheels differ in position less than $\Delta x=0.2 \Lambda$ and carry the same topological charge. If no corresponding pinwheel was found within $\Delta x$ it was considered as annihilated. If a pinwheel at $T_{i+1}$ could not be assigned to one at $T_{i}$ it was considered as created. We define the pinwheel creation $c(t)$ and annihilation $a(t)$ rates per hypercolumn as

$$
c(t)=\frac{d N_{c}}{\Lambda^{2} d t}, \quad a(t)=\frac{d N_{a}}{\Lambda^{2} d t},
$$

where $N_{c}$ and $N_{a}$ are the numbers of created and annihilated pinwheels. Creation and annihilation rates were confirmed by doubling the number of time frames.

To what extent are the pinwheels of the final pattern just rearrangements of pinwheels at some given time $T$ ? To answer this question for a given set of pinwheels at an initial time $T=T^{*}$ we further calculate the fraction $s(t)$ of those pinwheels surviving until time $T$. Finally, the fraction of pinwheels present at time $T^{*}$ that survive up to the final time $T=T_{f}$ is given by $p(t)$. 


\section{Pinwheel Stabilization by Inter-Map Coupling}

\subsection{Introduction}

In this chapter, we apply our general model for the coordinated development of OP and OD maps to study whether inter-map coupling is able to stabilize pinwheels which would become unstable in the weak coupling limit. In the previous chapter, we identified coupling energies which can be expected to account for the geometric correlations between OD and OP maps as observed in experiments. We study the impact these couplings actually have on the layout of the OP map and its relation to the OD map. Because the contralateral eye dominates during the initial formation of OD columns [57, 58, we systematically study the impact of overall dominance by one eye on the dynamics of pinwheels. We show that for the low order inter-map coupling energies there is for large inter-map coupling strength a suppression of the OP pattern leading to a complete loss of orientation selectivity. In contrast, for the higher order inter-map coupling energies, orientation selectivity is preserved for arbitrary strong coupling. We identified a limit in which the map interactions become unidirectional and in which we can neglect the backreaction onto the amplitudes of the OD map. This enables us to comprehensively analyze the existence and stability of pinwheel free and pinwheel rich OP maps in the coupled system. We identify a class of uniform solutions to the amplitude equations that depends on a single parameter which is specific to the considered coupling energy. These uniform solutions are pinwheel rich and have hexagonal spatial layout. In addition we identify non-uniform, pinwheel rich solutions with rhombic spatial layout. We calculate the stability properties and phase diagrams of these and additional pinwheel free solutions as a function of inter-map coupling and contralateral eye dominance. It turns out that pinwheel crystals can be stabilized above a critical degree of contralateral eye dominance. We confirm these predictions by solving the full field dynamics numerically. In particular, we show that an initially pinwheel free stripe pattern would evolve towards a pinwheel rich solution by a pinwheel creation process. Finally, we extend our model with additional visual cortical maps and assess the effects of detuning of OD and OP average wavelength. The coupled dynamics of OP and OD maps we consider in this chapter is given by

$$
\begin{aligned}
& \partial_{t} z(\mathbf{x}, t)=\hat{L}_{z} z(\mathbf{x}, t)-|z|^{2} z-\frac{\delta U}{\delta \bar{z}} \\
& \partial_{t} o(\mathbf{x}, t)=\hat{L}_{o} o(\mathbf{x}, t)-o(\mathbf{x}, t)^{3}-\frac{\delta U}{\delta o}+\gamma
\end{aligned}
$$


Here $\hat{L}_{\{o, z\}}=r_{\{o, z\}}-\left(k_{c}^{2}+\Delta\right)^{2}, \Lambda=2 \pi / k_{c}$. We break inversion symmetry explicitly with the constant $\gamma$ which leads for $\gamma>0$ to an over-representation of the contralateral eye input. The inter-map coupling energies $U$ are given in Eq. (3.23). The dynamics can be derived from an energy functional as

$$
\partial_{t} z(\mathbf{x})=-\frac{\delta E[z, o]}{\delta \bar{z}(\mathbf{x})}, \quad \partial_{t} o(\mathbf{x})=-\frac{\delta E[z, o]}{\delta o(\mathbf{x})}
$$

with

$$
\begin{aligned}
E[z, o]= & -\int d^{2} x\left(\bar{z}(\mathbf{x}) \hat{L}_{z} z(\mathbf{x})+\frac{1}{2} o(\mathbf{x}) \hat{L}_{o} o(\mathbf{x})-\frac{1}{2}|z(\mathbf{x})|^{4}-\frac{1}{4} o(\mathbf{x})^{4}\right) \\
& -\gamma o(\mathbf{x})+\int d^{2} x U
\end{aligned}
$$

In this model pinwheels are unstable in the weak coupling limit leading to systems of OP stripes thus mimicking the behavior of competitive Hebbian models for OD or OP maps in this situation 32 .

\subsection{The transition from OD stripes to OD blobs}

We first study how the emerging OD map depends on the overall eye dominance. To this end we map the uncoupled OD dynamics to a Swift-Hohenberg equation containing a quadratic interaction term instead of a constant bias. This allows the use of weakly nonlinear analysis to derive amplitude equations as an approximate description of the shifted OD dynamics near the bifurcation point. We identify the stationary solutions and study their stability properties. Finally, we derive expressions for the fraction of contralateral eye dominance for the stable solutions.

\subsubsection{Mapping to a dynamics with a quadratic nonlinearity}

Here we describe how to map the Swift-Hohenberg equation

$$
\partial_{t} o(\mathbf{x}, t)=\hat{L} o(\mathbf{x}, t)-o(\mathbf{x}, t)^{3}+\gamma,
$$

to one with a quadratic interaction term. To eliminate the constant term we shift the field by a constant amount $o(\mathbf{x}, t)=\widetilde{o}(\mathbf{x}, t)+\delta$. This changes the linear and nonlinear terms as

$$
\begin{aligned}
\hat{L} o & \rightarrow \hat{L} \widetilde{o}-\left(k_{c}^{4}-r_{o}\right) \delta \\
o^{3} & \rightarrow-\widetilde{o}^{3}+3 \delta \widetilde{o}^{2}+3 \delta^{2} \widetilde{o}+\delta^{3} .
\end{aligned}
$$

We define the new parameters $\widetilde{r}_{o}=r_{o}-3 \delta^{2}$ and $\widetilde{\gamma}=-3 \delta$. This leads to the new dynamics

$$
\partial_{t} \widetilde{o}=\widetilde{r}_{o} \widetilde{o}-\left(k_{c}^{2}+\Delta\right)^{2} \tilde{o}+\tilde{\gamma} \widetilde{o}^{2}-\widetilde{o}^{3}-\delta^{3}-\left(k_{c, o}^{4}-r_{o}\right) \delta+\gamma
$$


The condition that the constant part is zero is thus given by

$$
-\delta^{3}-\left(k_{c}^{4}-r_{o}\right) \delta+\gamma=0
$$

For $r_{o}<1$ the real solution to Eq. (4.7) is given by

$$
\delta=\frac{2^{1 / 3}\left(k_{c}-r_{o}\right)}{\beta}-\frac{\beta}{32^{1 / 3}},
$$

with $\beta=\left(-27 \gamma+\sqrt{108\left(r_{o}-k_{c}\right)^{3}+729 \gamma^{2}}\right)^{1 / 3}$. For small $\gamma$ this formula is approximated as

$$
\delta \approx \gamma \frac{1}{k_{c}^{4}-r_{o}}-\gamma^{3} \frac{1}{\left(k_{c}^{4}-r_{o}\right)^{4}}+3 \gamma^{5} \frac{1}{\left(k_{c}^{4}-r_{o}\right)^{7}}+\ldots
$$

The uncoupled OD dynamics we consider in the following is therefore given by

$$
\partial_{t} \widetilde{o}=\widetilde{r}_{o} \widetilde{o}-\left(k_{c}^{2}+\Delta\right)^{2} \widetilde{o}+\widetilde{\gamma} \widetilde{o}^{2}-\widetilde{o}^{3} .
$$

This equation has been extensively studied in pattern formation literature [13.

\subsubsection{Amplitude equations for OD patterns}

We study Eq. (4.10) using weakly nonlinear analysis. This method leads to amplitude equation as an approximate description of the full field dynamics near the bifurcation point $\widetilde{r}_{o}=0$. We summarize the derivation of the amplitude equations for the OD dynamics which is of the form

$$
\partial_{t} o(\mathbf{x}, t)=\hat{L} o(\mathbf{x}, t)+N_{2}[o, o]-N_{3}[o, o, o],
$$

with the linear operator $\hat{L}=r_{o}-\left(k_{c, o}^{2}+\Delta\right)^{2}$. In this section we use for simplicity the variables $\left(o, r_{o}, \gamma\right)$ instead of $\left(\tilde{o}, \tilde{r}_{o}, \tilde{\gamma}\right)$. The derivation is performed for general quadratic and cubic nonlinearities but is specified later according to Eq. (4.1) as $N_{3}[o, o, o]=o^{3}$ and $N_{2}[o, o]=\gamma o^{2}$. For the calculations in the following, it is useful to separate $r_{o}$ from the linear operator

$$
\hat{L}=r_{o}+\hat{L}^{0}
$$

therefore the largest eigenvalue of $\hat{L}^{0}$ is zero. The amplitude of the field $o(\mathbf{x}, t)$ is assumed to be small near the onset $r_{o}=0$ and thus the nonlinearities are small. We therefore expand both the field $o(\mathbf{x}, t)$ and the control parameter $r_{o}$ in powers of a small expansion parameter $\mu$ as

$$
o(\mathbf{x}, t)=\mu o_{1}(\mathbf{x}, t)+\mu^{2} o_{2}(\mathbf{x}, t)+\mu^{3} o_{3}(\mathbf{x}, t)+\ldots,
$$

and

$$
r_{o}=\mu r_{1}+\mu^{2} r_{2}+\mu^{3} r_{3}+\ldots
$$


The dynamics at the critical point $r_{o}=0$ becomes arbitrarily slow since the intrinsic timescale $\tau=r_{o}^{-1}$ diverges at the critical point. To compensate we introduce a rescaled time scale $T$ as

$$
T=r_{o} t, \quad \partial_{t}=r_{o} \partial_{T}
$$

In order for all terms in Eq. (4.11) to be of the same order the quadratic interaction term $N_{2}$ must be small. We therefore rescale $N_{2}$ as $\sqrt{r_{o}} N_{2}$. This preserves the nature of the bifurcation compared to the case $N_{2}=0$.

We insert the expansion Eq. (4.13) and Eq. (4.14) into the dynamics Eq. (4.11) and get

$$
\begin{aligned}
0 & =\mu \hat{L}^{0} o_{1} \\
& +\mu^{2}\left(-\hat{L}^{0} o_{2}-r_{1} \partial_{T} o_{1}+r_{1} o_{1}+\sqrt{\mu r_{1}+\mu^{2} r_{2}+\ldots} N_{2}\left[o_{1}, o_{1}\right]\right) \\
& +\mu^{3}\left(-\hat{L}^{0} o_{3}+r_{1}\left(o_{2}-\partial_{T} o_{2}\right)+r_{2}\left(o_{1}-\partial_{T} o_{1}\right)-N_{3}\left[o_{1}, o_{1}, o_{1}\right]\right) \\
& \vdots
\end{aligned}
$$

We sort and collect all terms in order of their power in $\mu$. The equation can be fulfilled for $\mu>0$ only if each of these terms is zero. We therefore solve the equation order by order. In the leading order we get the homogeneous equation

$$
\hat{L}^{0} o_{1}=0
$$

Thus $o_{1}$ is an element of the kernel of $\hat{L}^{0}$. The kernel contains linear combinations of modes with wavevector $\vec{k}_{j}$ on the critical circle $\left|\vec{k}_{j}\right|=k_{c, o}$. At this level any of such wavevectors is possible. We choose

$$
o_{1}=\sum_{j}^{n} B_{j}(T) e^{i \vec{k}_{j} \vec{x}}+\sum_{j}^{n} \bar{B}_{j}(T) e^{-i \vec{k}_{j} \vec{x}},
$$

where the wavevectors are chosen to be equally spaced $\vec{k}_{j}=k_{c, o}(\cos (j \pi / n), \sin (j \pi / n))$ and the complex amplitudes $B_{j}=\mathcal{B}_{j} e^{\imath \psi_{j}}$. The homogeneous equation leaves the amplitudes $B_{j}$ undetermined. These amplitudes are fixed by the higher order equations. Besides the leading order homogeneous equation we get inhomogeneous equations of the form

$$
\hat{L}^{0} o_{m}=F_{m} \text {. }
$$

To solve this inhomogeneous equation we apply a solvability condition known as the Fredholm alternative theorem. Since the operator $\hat{L}^{0}$ is self-adjoint, $\hat{L}^{0}=\hat{L}^{0 \dagger}$, the equation is solvable if and only if $F_{m}$ is orthogonal to the kernel of $\hat{L}^{0}$ i.e.

$$
\left\langle F_{m}, \widetilde{o}\right\rangle=0, \quad \forall \hat{L}^{0} \widetilde{o}=0
$$

The orthogonality to the kernel can be expressed by a projection operator $\hat{P}_{c}$ onto the kernel and the condition $\langle F, \widetilde{o}\rangle=0$ can be rewritten as $\hat{P}_{c} F=0$. 
At second order we get

$$
\hat{L}^{0} o_{2}=r_{1}\left(o_{1}-\partial_{T} o_{1}\right)
$$

Applying the solvability condition Eq. (4.20) we see that this equation can be fulfilled only for $r_{1}=0$. At third order we get

$$
\hat{L}^{0} o_{3}=r_{2}\left(o_{1}-\partial_{T} o_{1}\right)+N_{2}\left[o_{1}, o_{1}\right]-N_{3}\left[o_{1}, o_{1}, o_{1}\right]
$$

The parameter $r_{2}$ sets the scale in which $o_{1}$ is measured and we can set $r_{2}=1$. We apply the solvability condition and get

$$
\partial_{T} o_{1}=o_{1}+\hat{P}_{c} N_{2}\left[o_{1}, o_{1}\right]-\hat{P}_{c} N_{3}\left[o_{1}, o_{1}, o_{1}\right]
$$

We insert our ansatz Eq. (4.18) which leads to the amplitude equations at third order

$$
\partial_{T} B_{i}=B_{i}+\hat{P}_{i} \sum_{j, k} B_{j} B_{k} e^{-\imath \vec{k}_{i} \vec{x}} N_{2}\left[e^{\imath \vec{k}_{j} \vec{x}}, e^{\imath \vec{k}_{k} \vec{x}}\right]-\hat{P}_{i} \sum_{j, k} B_{j} B_{k} B_{l} e^{-\imath \vec{k}_{i} \vec{x}} N_{3}\left[e^{\imath \vec{k}_{j} \vec{x}}, e^{\imath \vec{k}_{k} \vec{x}}, e^{\imath \vec{k}_{l} \vec{x}}\right]
$$

where $\hat{P}_{i}$ is the projection operator onto the subspace $\left\{e^{\imath \vec{k}_{i} \vec{x}}\right\}$ of the kernel. $\hat{P}_{i}$ picks out all combinations of the modes which have their wavevector equal to $\vec{k}_{i}$. In our case the three active modes form a so called triad resonance $\vec{k}_{1}+\vec{k}_{2}+\vec{k}_{3}=0$. The quadratic coupling terms which are resonant to the mode $B_{1}$ are therefore given by

$$
\bar{B}_{2} \bar{B}_{3} e^{-\imath \vec{k}_{1} \vec{x}}\left(N_{2}\left[e^{-\imath \vec{k}_{2} \vec{x}}, e^{-\imath \vec{k}_{3} \vec{x}}\right]+N_{2}\left[e^{-\imath \vec{k}_{3} \vec{x}}, e^{-\imath \vec{k}_{2} \vec{x}}\right]\right) .
$$

Resonant contributions from the cubic nonlinearity result from terms of the form $\left|B_{j}\right|^{2} B_{i}$. Their coupling coefficients are given by

$$
\begin{aligned}
\widetilde{g}_{i j}= & N_{3}\left[e^{l \vec{k}_{i} \vec{x}}, e^{i \vec{k}_{j} \vec{x}}, e^{-\imath \vec{k}_{j} \vec{x}}\right]+N_{3}\left[e^{l \vec{k}_{i} \vec{x}}, e^{-\imath \vec{k}_{j} \vec{x}}, e^{\imath \vec{k}_{j} \vec{x}}\right]+N_{3}\left[e^{\imath \vec{k}_{j} \vec{x}}, e^{\imath \vec{k}_{i} \vec{x}}, e^{-\imath \vec{k}_{j} \vec{x}}\right]+ \\
& N_{3}\left[e^{-\imath \vec{k}_{j} \vec{x}}, e^{\imath \vec{k}_{i} \vec{x}}, e^{\imath \vec{k}_{j} \vec{x}}\right]+N_{3}\left[e^{\imath \vec{k}_{j} \vec{x}}, e^{-\imath \vec{k}_{j} \vec{x}}, e^{\imath \vec{k}_{i} \vec{x}}\right]+N_{3}\left[e^{-\imath \vec{k}_{j} \vec{x}}, e^{\imath \vec{k}_{j} \vec{x}}, e^{\imath \vec{k}_{i} \vec{x}}\right]
\end{aligned}
$$

and

$$
\widetilde{g}_{i i}=N_{3}\left[e^{\imath \vec{k}_{i} \vec{x}}, e^{\imath \vec{k}_{i} \vec{x}}, e^{-\imath \vec{k}_{i} \vec{x}}\right]+N_{3}\left[e^{\imath \vec{k}_{i} \vec{x}}, e^{-\imath \vec{k}_{i} \vec{x}}, e^{\imath \vec{k}_{i} \vec{x}}\right]+N_{3}\left[e^{-\imath \vec{k}_{i} \vec{x}}, e^{\imath \vec{k}_{i} \vec{x}}, e^{\imath \vec{k}_{i} \vec{x}}\right]
$$

When specifying the nonlinearities Eq. (4.1) the coupling coefficients are given by $\widetilde{g}_{i j}=6, \widetilde{g}_{i i}=$ 3. Finally, the amplitude equations (here in the shifted variables $\left(\tilde{r}_{o}, \tilde{\gamma}\right)$ are given by

$$
\partial_{t} B_{1}=\widetilde{r}_{o} B_{1}-3\left|B_{1}\right|^{2} B_{1}-6\left(\left|B_{2}\right|^{2}+\left|B_{3}\right|^{2}\right) B_{1}+2 \widetilde{\gamma} \bar{B}_{2} \bar{B}_{3}
$$

where we scaled back to the original time variable $t$. Equations for $B_{2}$ and $B_{3}$ are given by cyclic permutation of the indices. 


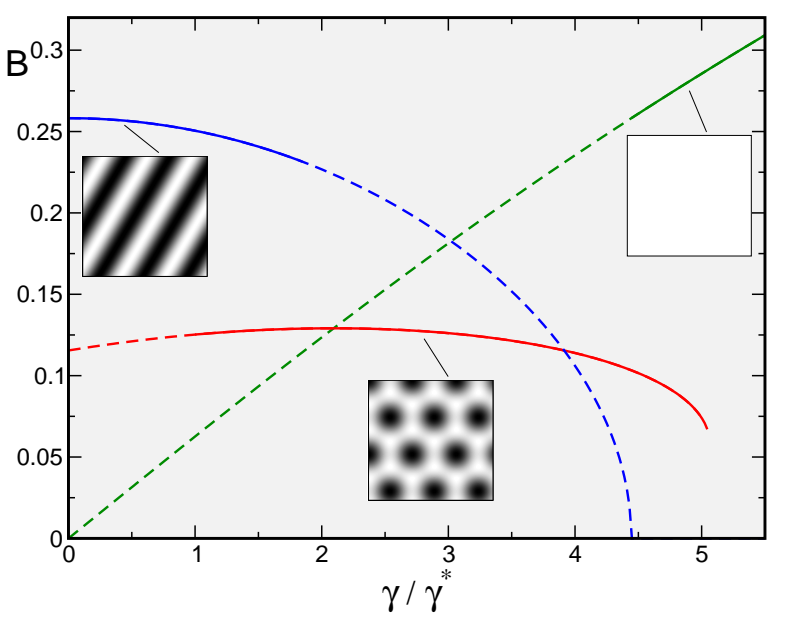

Figure 4.1: Stationary amplitudes of the OD dynamics. The course of $\mathcal{B}_{s t}(\gamma)$ Eq. (4.29) (blue), $\mathcal{B}_{\text {hex }}(\gamma)$ Eq. (4.30) (red), and of $\delta(\gamma)$ Eq. (4.8) (green) for $r_{o}=0.2$. The solutions are plotted in solid lines within their stability ranges.

\subsubsection{Stationary solutions}

The amplitude equations (4.28) have three types of stationary solutions namely ocular dominance stripes

$$
o_{s t}(\mathbf{x})=2 \mathcal{B}_{s t} \cos (x+\psi)+\delta
$$

with $\mathcal{B}_{s t}=\sqrt{\tilde{r} / 3}$, hexagons

$$
o_{\text {hex }}(\mathbf{x})=\mathcal{B}_{\text {hex }} \sum_{j=1}^{3} e^{\imath \psi_{j}} e^{\imath \vec{k}_{j} \vec{x}}+\text { c.c. }+\delta
$$

with the resonance condition $\sum_{j}^{3} \vec{k}_{j}=0$ and $\mathcal{B}_{\text {hex }}=-\tilde{\gamma} / 15+\sqrt{(\tilde{\gamma} / 15)^{2}+\tilde{r} / 15}$. Finally, there is a homogeneous solution with spatially constant eye dominance

$$
o_{c}(\mathbf{x})=\delta
$$

The spatial average of all solutions is $\langle o(\mathbf{x})\rangle=\delta$. The course of $\mathcal{B}_{\text {st }}, \mathcal{B}_{\text {hex }}$, and of $\delta(\gamma)$ is shown in Fig. 4.1.

\subsubsection{Linear stability analysis for OD patterns}

We decompose the amplitude equations (4.28) into the real and imaginary parts. From the imaginary part we get the phase equation

$$
\partial_{t} \psi_{1}=-2 \widetilde{\gamma} \sin \left(\psi_{1}+\psi_{2}+\psi_{3}\right)
$$

and equations for $\psi_{2}, \psi_{3}$ by cyclic permutation of the indices. The stationary phases are given by $\psi_{1}+\psi_{2}+\psi_{3}=\{0, \pi\}$. The phase equation can be derived from the potential $V[\psi]=-2 \widetilde{\gamma} \cos \left(\psi_{1}+\psi_{2}+\psi_{3}\right)$. We see that the solution $\psi_{1}+\psi_{2}+\psi_{3}=0$ is stable for $\widetilde{\gamma}>0(\gamma<0)$ and the solution $\psi_{1}+\psi_{2}+\psi_{3}=\pi$ is stable for $\widetilde{\gamma}<0(\gamma>0)$.

We calculate the stability borders of the OD stripe, hexagon, and constant solution in the uncoupled case. This treatment follows [13]. In case of stripes the three modes of the amplitude 
equations are perturbed as

$$
B_{1} \rightarrow B_{s t}+b_{1}, \quad B_{2} \rightarrow b_{2}, \quad B_{3} \rightarrow b_{3},
$$

assuming small perturbations $b_{1}, b_{2}$, and $b_{3}$. This leads to the linear equations $\partial_{t} \vec{b}=M \vec{b}$ with the stability matrix

$$
M=\left(\begin{array}{ccc}
\widetilde{r}-9 B_{s t}^{2} & 0 & 0 \\
0 & \widetilde{r}-6 B_{s t}^{2} & 2 \widetilde{\gamma} B_{s t} \\
0 & 2 \widetilde{\gamma} B_{s t} & \widetilde{r}-6 B_{s t}^{2}
\end{array}\right)
$$

The corresponding eigenvalues are given by

$$
\lambda=(-2 \widetilde{r},-\widetilde{r}-2 \sqrt{\widetilde{r} / 3} \widetilde{\gamma},-\widetilde{r}+2 \sqrt{\widetilde{r} / 3} \widetilde{\gamma})
$$

This leads to the two borders for the stripe stability

$$
\widetilde{r}=0, \quad \widetilde{r}=\frac{4}{3} \widetilde{\gamma}^{2} .
$$

In terms of the original variables $r_{o}, \gamma$ the borders are given by $\left(0<r_{o}<1\right)$

$$
\gamma_{3}^{*}=\frac{\left(3-2 r_{o}\right) \sqrt{r_{o}}}{3^{3 / 2}}, \quad \gamma_{2}^{*}=\frac{\left(15-14 r_{o}\right) \sqrt{r_{o}}}{15^{3 / 2}} .
$$

To derive the stability borders for the hexagon solution $o_{\text {hex }}(\mathbf{x})$ we perturb the amplitudes as

$$
B_{1} \rightarrow B_{\text {hex }}+b_{1}, \quad B_{2} \rightarrow B_{\text {hex }}+b_{2}, \quad B_{3} \rightarrow B_{\text {hex }}+b_{3} .
$$

The stability matrix is then given by

$$
M=\left(\begin{array}{ccc}
-21 B_{\text {hex }}+\widetilde{\gamma} & -12 B_{\text {hex }}^{2}-2 B_{\text {hex }} \widetilde{\gamma} & -12 B_{\text {hex }}^{2}-2 B_{\text {hex }} \widetilde{\gamma} \\
-12 B_{\text {hex }}^{2}-2 B_{\text {hex }} \widetilde{\gamma} & -21 B_{\text {hex }}+\widetilde{r} & -12 B_{\text {hex }}^{2}-2 B_{\text {hex }} \widetilde{\gamma} \\
-12 B_{\text {hex }}^{2}-2 B_{\text {hex }} \widetilde{\gamma} & -12 B_{\text {hex }}^{2}-2 B_{\text {hex }} \widetilde{\gamma} & -21 B_{\text {hex }}+\widetilde{r}
\end{array}\right),
$$

and the corresponding eigenvalues are given by

$$
\lambda=\left(-45 B_{h}^{2}+\widetilde{r}-4 B_{h} \widetilde{\gamma},-9 B_{h}^{2}+\widetilde{r}+2 B_{h} \widetilde{\gamma},-9 B_{h}^{2}+\widetilde{r}+2 B_{h} \widetilde{\gamma}\right) .
$$

The stability borders for the hexagon solution are given by

$$
\widetilde{r}=-\frac{1}{15} \widetilde{\gamma}^{2}, \quad \widetilde{r}=\frac{16}{3} \widetilde{\gamma}^{2} .
$$

In terms of the original variables we finally get

$$
\gamma_{4}^{*}=\frac{\left(12-7 r_{o}\right) \sqrt{r_{o}} \sqrt{5}}{24 \sqrt{3}}, \quad \gamma^{*}=\frac{\left(51-50 r_{o}\right) \sqrt{r_{o}}}{51^{3 / 2}} .
$$

The phase diagram of this model is depicted in Fig. 4.2(a). It shows the stability borders 
(a)

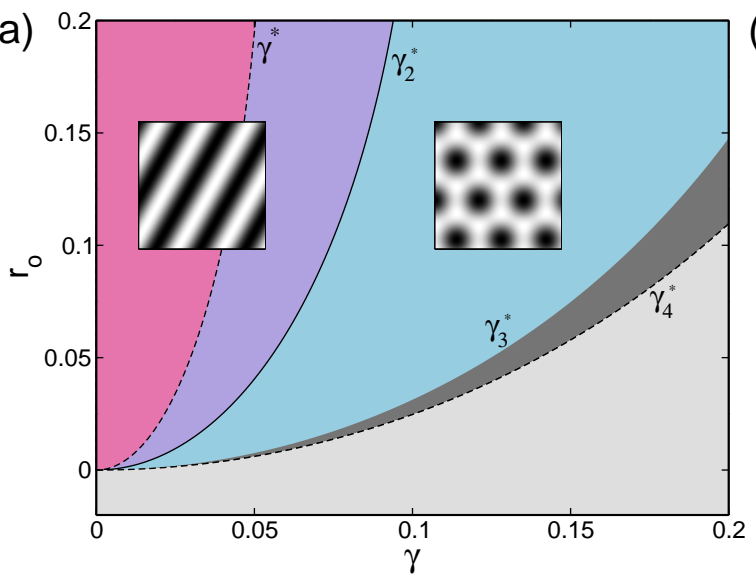

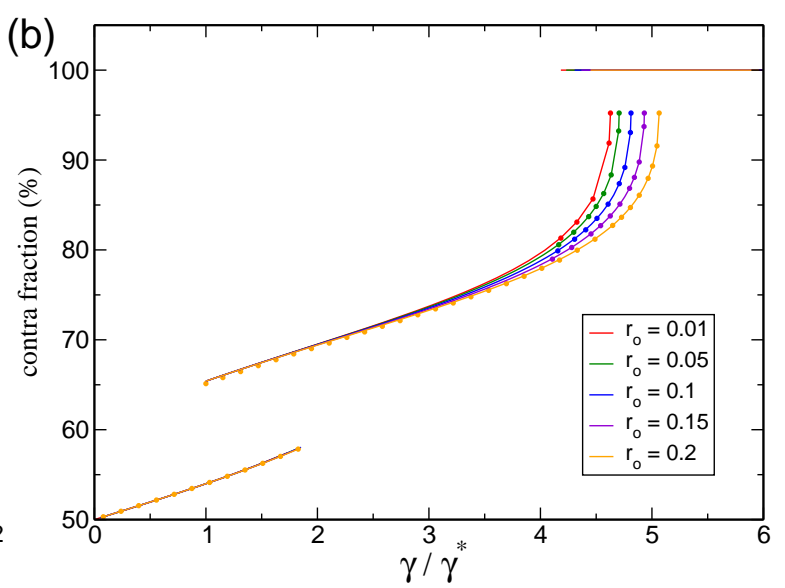

Figure 4.2.: The uncoupled OD dynamics. (a) Phase diagram of the OD model Eq. (4.1). Dashed lines: stability border of hexagon solutions, solid line: stability border of stripe solution, gray regions: stability region of constant solution (b) Percentage of neurons dominated by the contralateral eye plotted for the three stationary solutions. Circles: numerically obtained values, solid lines: $C_{s t}$ and $C_{\text {hex }}$.

$\gamma^{*}, \gamma_{2}^{*}, \gamma_{3}^{*}$, and $\gamma_{4}^{*}$ for the three solutions obtained by linear stability analysis. Without a bias term the OD map is either constant, for $r_{o}<0$, or has a stripe layout, for $r_{o}>0$. For positive $r_{o}$ and increasing bias term there are two transition regions, first a transition region from stripes to hexagons and second a transition region from hexagons to the constant solution.

The spatial layout of the OD hexagons consists of hexagonal arrays of ipsilateral eye dominance blobs in a sea of contralateral eye dominance, see Fig. 4.2(a).

\subsubsection{Contralateral eye fraction}

To compare the obtained solutions with physiological OD maps we next quantify the fraction of neurons selective to the contralateral eye inputs. For stripe and hexagon solutions we thus calculate the fraction of contralateral eye dominated territory $C_{s t}$ and $C_{h e x}$. In case of stripes this is a purely one-dimensional problem. The zeros of the field are given by

$$
o_{s t}(x)=2 \mathcal{B}_{s t} \cos (x+\psi)+\delta=0,
$$

with the solution

$$
x=\arccos \left(\frac{-\delta}{2 \mathcal{B}_{s t}}\right) .
$$

As the field has a periodicity of $\pi$ the area fraction is given by

$$
C_{s t}=\arccos \left(\frac{-\delta}{2 \mathcal{B}_{s t}}\right) / \pi
$$

In case of hexagons we observe that the territory of negative $o(\mathbf{x})$ values is approximately a circular area. We obtain the fraction of negative $o(\mathbf{x})$ values by relating this area to the area 
of the whole hexagonal lattice. In case of hexagons the field is given by

$$
o_{\text {hex }}(\mathbf{x})=2 \mathcal{B}_{\text {hex }} \sum_{j} \cos \left(\vec{k}_{j} \vec{x}+\psi_{j}\right)+\delta .
$$

As an approximation we project the field onto the $x$-axis and choose for simplicity $\psi_{j}=0, \forall j$. The field has its maximum at the origin $o_{h e x}(0,0)=6+\delta$. The projection leads to

$$
f(x)=2 \mathcal{B}_{\text {hex }}(\cos x+2 \cos (1 / 2) x)+\delta .
$$

The zeros $f\left(x_{1}\right)=0$ are located at

$$
x_{1}=2 \arccos \left(\frac{1}{2}\left(-1+\sqrt{3+\frac{\delta}{\mathcal{B}_{\text {hex }}}}\right)\right) .
$$

The circular area of positive $o_{h e x}(\mathbf{x})$ values is now given by $A_{c}=\pi x_{1}^{2}$. The periodicity of the hexagonal pattern is given by $f\left(x_{2}\right)_{\delta=0}=\min (f)_{\delta=0}=-3$. This leads to $x_{2}=4 \pi / 3$. The area of the hexagon is therefore given by $A_{\text {hex }}=3 x_{2}^{2} \sqrt{3} / 2$. The contra fraction is finally given by

$$
1-C_{\text {hex }} \approx \frac{A_{c}}{A_{\text {hex }}}=\frac{\sqrt{3}}{2 \pi} \arccos \left(\frac{1}{2}\left(-1+\sqrt{3+\frac{\delta}{\mathcal{B}_{\text {hex }}}}\right)\right)^{2} .
$$

The course of the fractions $C_{s t}$ and $C_{\text {hex }}$ is shown in Fig. 4.2(b). At the border $\gamma=\gamma^{*}$, where hexagons become stable $C_{\text {hex }} \approx 65.4 \%$. At the border $\gamma=\gamma_{4}^{*}$, where hexagons lose stability $C_{\text {hex }} \approx 95.2 \%$. Both quantities are independent of $r_{o}$. We confirmed our results by direct numerical calculation of the fraction of positive $o_{h e x}(\mathbf{x})$ pixel values. Deviations from the result Eq. (4.49) are small. For $\gamma / \gamma^{*} \approx 1$ the zeros of Eq. (4.47) are not that well approximated with a circular shape and the projection described above leads to the small deviations which decrease with increasing bias $\gamma$.

\subsection{Coupled amplitude equations}

We next study the influence of the OD patterns on the dynamics of the OP map. To this end we derive coupled amplitude equations for the fields $z(\mathbf{x}, t)$ and $o(\mathbf{x}, t)$. We expand both fields in powers of the small expansion parameter $\mu$. To account for the fact that one bifurcation parameter field can be substantially smaller than the other we expand the fields as

$$
\begin{aligned}
& o(\mathbf{x}, t)=\mu o_{1}(\mathbf{x}, t)+\mu^{2} o_{2}(\mathbf{x}, t)+\mu^{3} o_{3}(\mathbf{x}, t)+\ldots \\
& z(\mathbf{x}, t)=(\kappa \mu) z_{1}(\mathbf{x}, t)+(\kappa \mu)^{2} z_{2}(\mathbf{x}, t)+(\kappa \mu)^{3} z_{3}(\mathbf{x}, t)+\ldots
\end{aligned}
$$


We further expand both control parameters as

$$
\begin{aligned}
& r_{o}=\mu \tilde{r}_{1}+\mu^{2} \tilde{r}_{2}+\mu^{3} \tilde{r}_{3}+\ldots \\
& r_{z}=(\kappa \mu) r_{1}+(\kappa \mu)^{2} r_{2}+(\kappa \mu)^{3} r_{3}+\ldots
\end{aligned}
$$

We introduce a common slow timescale $T=r_{z} t$ and insert the expansions in Eq. (4.1) and get

$$
\begin{aligned}
0= & \mu \kappa \hat{L}^{0} z_{1} \\
& +\mu^{2} \kappa^{2}\left(-\hat{L}^{0} z_{2}+r_{1} z_{1}-r_{1} \partial_{T} z_{1}\right) \\
& +\mu^{3} \kappa^{3}\left(-r_{2} \partial_{T} z_{1}+r_{2} z_{1}+r_{1} z_{2}-r_{1} \partial_{T} z_{2}-\hat{L}^{0} z_{3}-N_{3, u}\left[z_{1}, z_{1}, \bar{z}_{1}\right]\right) \\
& +\mu^{3} \kappa\left(-\alpha N_{3, c}\left[z_{1}, o_{1}, o_{1}\right]\right) \\
& \vdots \\
& +\mu^{7} \kappa^{7}\left(-\hat{L}^{0} z_{7}+r_{2} z_{5}+r_{4} z_{3}+r_{6} z_{1}+\cdots-N_{3, u}\left[z_{3}, z_{1}, \bar{z}_{3}\right]+N_{3, u}\left[z_{5}, z_{1}, \bar{z}_{1}\right]+\ldots\right) \\
& +\mu^{7}\left(-\alpha \kappa^{5} N_{3, c}\left[z_{5}, o_{1}, o_{1}\right]-\kappa^{3} \alpha N_{3, c}\left[z_{3}, o_{3}, o_{1}\right]-\ldots\right) \\
& +\mu^{7} \kappa^{3}\left(-\epsilon N_{7, c}\left[z_{1}, z_{1}, \bar{z}_{1}, o_{1}, o_{1}, o_{1}, o_{1}\right]\right) \\
& \vdots
\end{aligned}
$$

and

$$
\begin{aligned}
0= & \mu \hat{L}^{0} o_{1} \\
& +\mu^{2}\left(-\hat{L}^{0} o_{2}+\tilde{r}_{1} o_{1}-\kappa r_{1} \partial_{T} o_{1}+\sqrt{\left.\mu \tilde{r}_{1}+\mu^{2} \tilde{r}_{2}+\ldots \tilde{N}_{2, u}\left[o_{1}, o_{1}\right]\right)}\right. \\
& +\mu^{3}\left(-\kappa^{2} r_{2} \partial_{T} o_{1}+\tilde{r}_{2} o_{1}+\tilde{r}_{1} o_{2}-\kappa r_{1} \partial_{T} o_{2}-\hat{L}^{0} o_{3}-\tilde{N}_{3, u}\left[o_{1}, o_{1}, o_{1}\right]\right) \\
& +\mu^{3}\left(-\kappa^{2} \alpha \tilde{N}_{3, c}\left[z_{1}, \bar{z}_{1}, o_{1}\right]\right) \\
& \vdots \\
& +\mu^{7}\left(-\hat{L}^{0} o_{7}+\tilde{r}_{2} o_{5}+\tilde{r}_{4} o_{3}+\tilde{r}_{6} o_{1}+\cdots-\tilde{N}_{3, u}\left[o_{3}, o_{1}, o_{1}\right]-\tilde{N}_{2, u}\left[o_{1}, o_{5}\right]-\ldots\right) \\
& +\mu^{7}\left(-\kappa^{5} \alpha \tilde{N}_{3, c}\left[z_{5}, o_{1}, o_{1}\right]-\kappa^{3} \alpha \tilde{N}_{3, c}\left[z_{3}, o_{3}, o_{1}\right]-\ldots\right) \\
& +\mu^{7} \kappa^{4}\left(-\epsilon \tilde{N}_{7, c}\left[z_{1}, z_{1}, \bar{z}_{1}, \bar{z}_{1}, o_{1}, o_{1}, o_{1}\right]\right) \\
& \vdots
\end{aligned}
$$

with the nonlinearities $\tilde{N}_{2, u}=o^{2}, N_{3, u}=|z|^{2} z, \tilde{N}_{3, u}=o^{3}$, and $N_{3, c}, N_{7, c}, \tilde{N}_{3, c}, \tilde{N}_{7, c}$ the nonlinearities of the inter-map coupling energy Eq. (3.24).

We further scaled out the inter-map coupling strength $\alpha$ and $\epsilon$. We consider amplitude equations up to seventh order as this is the order where the nonlinearity of the higher order coupling energy enters first. For Eq. (4.52) and Eq. (4.53) to vanish each individual order in 
$\mu$ has to be zero. At linear order in $\mu$ we get the two homogeneous equations

$$
\hat{L}^{0} z_{1}=0, \quad \hat{L}^{0} o_{1}=0
$$

Thus $z_{1}$ and $o_{1}$ are elements of the kernel of $\hat{L}^{0}$. The kernel contains linear combinations of modes with a wavevector on the critical circle i.e.

$$
\begin{aligned}
& z_{1}(\mathbf{x}, T)=\sum_{j}^{n}\left(A_{j}^{(1)}(T) e^{\imath \vec{k}_{j} \vec{x}}+A_{j^{-}}^{(1)}(T) e^{-\imath \vec{k}_{j} \vec{x}}\right) \\
& o_{1}(\mathbf{x}, T)=\sum_{j}^{n}\left(B_{j}^{(1)}(T) e^{\imath \vec{k}_{j} \vec{x}}+\bar{B}_{j}^{(1)}(T) e^{-\imath \vec{k}_{j} \vec{x}}\right)
\end{aligned}
$$

with the complex amplitudes $A_{j}^{(1)}=\mathcal{A}_{j} e^{\imath \phi_{j}}, B_{j}^{(1)}=\mathcal{B}_{j} e^{\imath \psi_{j}}$. To account for the hexagonal layout of the OD pattern we choose $n=3$. Since in cat visual cortex the typical wavelength for OD and OP maps are approximately the same [55,79] i.e. $k_{c, o}=k_{c, z}$ the Fourier components of the emerging pattern are located on a common critical circle $\vec{k}_{j}=\vec{k}_{j}^{\prime}=\left(\cos \alpha_{j}, \sin \alpha_{j}\right) k_{c}$. In Section 4.6.2 we study pattern formation when detuning OP and OD typical wavelength. At second order in $\mu$ we get

$$
\begin{array}{r}
\hat{L}^{0} z_{2}+r_{1} z_{1}-r_{1} \partial_{T} z_{1}=0 \\
\hat{L}^{0} o_{2}+\tilde{r}_{1} o_{1}-\kappa r_{1} \partial_{T} o_{1}=0 .
\end{array}
$$

As $z_{1}$ and $o_{1}$ are elements of the kernel $r_{1}=\tilde{r}_{1}=0$. At third order, when applying the solvability condition Eq. (4.20), we get

$$
\begin{aligned}
r_{2} \partial_{T} z_{1} & =r_{2} z_{1}-\hat{P}_{c} N_{3, u}\left[z_{1}, z_{1}, \bar{z}_{1}\right]-\frac{\alpha}{\kappa^{2}} \hat{P}_{c} N_{3, c}\left[z_{1}, o_{1}, o_{1}\right] \\
\kappa^{2} r_{2} \partial_{T} o_{1} & =\tilde{r}_{2} o_{1}-\sqrt{\tilde{r}_{2}} \hat{P}_{c} \tilde{N}_{2, u}\left[o_{1}, o_{1}\right]-\hat{P}_{c} \tilde{N}_{3, u}\left[o_{1}, o_{1}, o_{1}\right]-\kappa^{2} \alpha \hat{P}_{c} \tilde{N}_{3, c}\left[z_{1}, \bar{z}_{1}, o_{1}\right] .
\end{aligned}
$$

We insert the leading order fields Eq. (4.55) and obtain the amplitude equations

$$
\begin{aligned}
r_{2} \partial_{T} A_{i}^{(1)} & =r_{2} A_{i}^{(1)}-\sum_{j} g_{i j}\left|A_{j}^{(1)}\right|^{2} A_{i}^{(1)}-\sum_{j} f_{i j} A_{j}^{(1)} A_{j^{-}}^{(1)} \bar{A}_{i^{-}}^{(1)}-\frac{\alpha}{\kappa^{2}} \sum_{j} h_{i j}\left|B_{j}^{(1)}\right|^{2} A_{i}^{(1)} \\
\kappa^{2} r_{2} \partial_{T} B_{i}^{(1)} & =\tilde{r}_{2} B_{i}^{(1)}-2 \sqrt{\tilde{r}_{2}} \bar{B}_{i+1}^{(1)} \bar{B}_{i+2}^{(1)}-\sum_{j} \widetilde{g}_{i j}\left|B_{j}^{(1)}\right|^{2} B_{i}^{(1)}-\alpha \kappa^{2} \sum_{j} h_{i j}\left|A_{j}^{(1)}\right|^{2} B_{i}^{(1)} .
\end{aligned}
$$

For simplicity we have written only the simplest coupling terms. Depending on the configuration of active modes additional contributions may enter the amplitude equations. In addition there are coupling terms which contain the constant $\delta$, see Section 4.2.1. The coupling coef- 
ficients are given by

$$
\begin{aligned}
g_{i j} & =e^{-\imath \vec{k}_{i} \vec{x}}\left(N_{3, u}\left[e^{\imath \vec{k}_{i} \vec{x}}, e^{\imath \vec{k}_{j} \vec{x}}, e^{-\imath \vec{k}_{j} \vec{x}}\right]+N_{3, u}\left[e^{\imath \vec{k}_{j} \vec{x}}, e^{\imath \vec{k}_{i} \vec{x}}, e^{-\imath \vec{k}_{j} \vec{x}}\right]\right) \\
g_{i i} & =e^{-\imath \vec{k}_{i} \vec{x}} N_{3, u}\left[e^{\imath \vec{k}_{i} \vec{x}}, e^{\imath \vec{k}_{i} \vec{x}}, e^{-\imath \vec{k}_{i} \vec{x}}\right] \\
g_{i j^{-}} & =e^{-\imath \vec{k}_{i} \vec{x}}\left(N_{3, u}\left[e^{\imath \vec{k}_{i} \vec{x}}, e^{-\imath \vec{k}_{j} \vec{x}}, e^{\imath \vec{k}_{j} \vec{x}}\right]+N_{3, u}\left[e^{-\imath \vec{k}_{j} \vec{x}}, e^{\imath \vec{k}_{i} \vec{x}}, e^{\imath \vec{k}_{j} \vec{x}}\right]\right) \\
f_{i j} & =e^{-\imath \vec{k}_{i} \vec{x}}\left(N_{3, u}\left[e^{\imath \vec{k}_{j} \vec{x}}, e^{-\imath \vec{k}_{j} \vec{x}}, e^{\imath \vec{k}_{i} \vec{x}}\right]+N_{3, u}\left[e^{-\imath \vec{k}_{j} \vec{x}}, e^{\imath \vec{k}_{j} \vec{x}}, e^{\imath \vec{k}_{i} \vec{x}}\right]\right) \\
f_{i i} & =0 \\
h_{i j} & =e^{-\imath \vec{k}_{i} \vec{x}} N_{3, c}\left[e^{\imath \vec{k}_{i} \vec{x}}, e^{\imath \vec{k}_{j} \vec{x}}, e^{-\imath \vec{k}_{j} \vec{x}}\right] \\
h_{i i} & =e^{-\imath \vec{k}_{i} \vec{x}} N_{3, c}\left[e^{\imath \vec{k}_{i} \vec{x}}, e^{\imath \vec{k}_{i} \vec{x}}, e^{-\imath \vec{k}_{i} \vec{x}}\right]
\end{aligned}
$$

while the coupling coefficients $\widetilde{g}_{i j}$ are given in Eq. (4.26). When we set $r_{2}=1 / \kappa^{2}$ and $\tilde{r}_{2}=1$ we get

$$
\begin{aligned}
\partial_{T} A_{i}^{(1)} & =\left(1-\alpha \sum_{j} h_{i j}\left|B_{j}^{(1)}\right|^{2}\right) A_{i}^{(1)}-\kappa^{2} \sum_{j}\left(g_{i j}\left|A_{j}^{(1)}\right|^{2} A_{i}^{(1)}+f_{i j} A_{j}^{(1)} A_{j^{-}}^{(1)} \bar{A}_{i^{-}}^{(1)}\right) \\
\partial_{T} B_{i}^{(1)} & =B_{i}^{(1)}-2 \bar{B}_{i+1}^{(1)} \bar{B}_{i+2}^{(1)}-\sum_{j} \tilde{g}_{i j}\left|B_{j}^{(1)}\right|^{2} B_{i}^{(1)}-\kappa^{2} \alpha \sum_{j} h_{i j}\left|A_{j}^{(1)}\right|^{2} B_{i}^{(1)}
\end{aligned}
$$

For $\kappa \rightarrow 0$ the inter-map coupling term in the dynamics of the modes $B^{(1)}$ vanishes, leading to the uncoupled OD dynamics. In the dynamics for the modes $A^{(1)}$ the uncoupled cubic nonlinearities vanishes in this limit. Thus the dynamics is governed by the linear terms only. Inter-map coupling thus influences the growth rate of the Fourier modes. For large enough inter-map coupling strength $\alpha$ the growth rate becomes negative. In this case only the homogeneous solution $A_{j}^{(1)}=0, \forall j$ is stable.

Alternatively, we can set $r_{2}=\tilde{r}_{2}=1$ in Eq. (4.58), scale back to the fast time $t$, and rescale the amplitudes as $A_{i}=\sqrt{r_{z}} A_{i}^{(1)}, B_{i}=\sqrt{r_{o}} B_{i}^{(1)}$. This leads to the amplitude equations

$$
\begin{aligned}
\partial_{t} A_{i} & =r_{z} A_{i}-\sum_{j} g_{i j}\left|A_{j}\right|^{2} A_{i}-\sum_{j} f_{i j} A_{j} A_{j^{-}} \bar{A}_{i^{-}}-\frac{\alpha}{\kappa^{2}} \frac{r_{z}}{r_{o}} \sum_{j} h_{i j}\left|B_{j}\right|^{2} A_{i} \\
\kappa^{2} \frac{r_{o}}{r_{z}} \partial_{t} B_{i} & =r_{o} B_{i}-2 \bar{B}_{i+1} \bar{B}_{i+2}-\sum_{j} \tilde{g}_{i j}\left|B_{j}\right|^{2} B_{i}-\alpha \kappa^{2} \frac{r_{o}}{r_{z}} \sum_{j} h_{i j}\left|A_{j}\right|^{2} B_{i} .
\end{aligned}
$$

Since $r_{z} / r_{o}=\kappa^{2}+\left(\kappa^{4} r_{4}-\kappa^{2} \tilde{r}_{4}\right) \mu^{2}+\ldots$ we get at leading order

$$
\begin{aligned}
\partial_{t} A_{i} & =\left(r_{z}-\alpha \sum_{j} h_{i j}\left|B_{j}\right|^{2}\right) A_{i}-\sum_{j} g_{i j}\left|A_{j}\right|^{2} A_{i}-\sum_{j} f_{i j} A_{j} A_{j-} \bar{A}_{i^{-}} \\
\partial_{t} B_{i} & =r_{o} B_{i}-2 \bar{B}_{i+1} \bar{B}_{i+2}-\sum_{j} \tilde{g}_{i j}\left|B_{j}\right|^{2} B_{i}-\alpha \sum_{j} h_{i j}\left|A_{j}\right|^{2} B_{i} .
\end{aligned}
$$


At higher order we obtain corrections to the uncoupled dynamics of $z(\mathbf{x}, t)$ and $o(\mathbf{x}, t)$. In addition we get corrections to the third order coupling energy terms. Finally, at seventh order enters the nonlinearity of the higher order coupling energy term. The amplitude equations up to seventh order are thus derived from

$$
\begin{aligned}
\kappa^{3} r_{2} \partial_{T} z_{1} & =\kappa^{3} r_{2} z_{1}-\kappa^{3} \hat{P} N_{3, u}\left[z_{1}, z_{1}, \bar{z}_{1}\right]-\kappa \alpha \hat{P} N_{3, c}\left[z_{1}, o_{1}, o_{1}\right] \\
\kappa^{5} r_{2} \partial_{T} z_{3} & =\kappa^{5} r_{2} z_{3}-\cdots-\kappa^{5} \hat{P} N_{3, u}\left[z_{1}, z_{1}, \overline{z_{3}}\right] \\
\kappa^{7} r_{2} \partial_{T} z_{5} & =\kappa^{7} r_{2} z_{5}-\cdots-\kappa^{7} \hat{P} N_{3, u}\left[z_{3}, z_{1}, \overline{z_{3}}\right]-\epsilon \kappa^{3} \hat{P} N_{7, c}\left[z_{1}, z_{1}, \bar{z}_{1}, o_{1}, o_{1}, o_{1}, o_{1}\right]
\end{aligned}
$$

and corresponding equations for the fields $o_{1}, o_{3}$, and $o_{5}$. The field $z_{1}$ is given in Eq. (4.55) and its amplitudes $A^{(1)}$ and $B^{(1)}$ are determined at third order. The field $z_{3}$ contains contributions from modes off the critical circle $z_{3, o f f},\left|\vec{k}_{o f f}\right| \neq k_{c}$ and on the critical circle i.e. $z_{3}=$ $z_{3, o f f}+\sum_{j}^{n}\left(A_{j}^{(3)}(T) e^{\imath \vec{k}_{j} \vec{x}}+A_{j^{-}}^{(3)}(T) e^{-\imath \vec{k}_{j} \vec{x}}\right)$. Its amplitude $A^{(3)}$ are determined at fifth order. The field $z_{5}$ also contains contributions from modes off the critical circle $z_{5, \text { off }}$ and on the critical circle i.e. $z_{5}=z_{5, \text { off }}+\sum_{j}^{n}\left(A_{j}^{(5)}(T) e^{\imath \vec{k}_{j} \vec{x}}+A_{j^{-}}^{(5)}(T) e^{-\imath \vec{k}_{j} \vec{x}}\right)$. Its amplitude $A^{(5)}$ are determined at seventh order. This leads to a series of amplitude equations

$$
\begin{aligned}
\kappa^{3} r_{2} \partial_{T} A_{i}^{(1)} & =\kappa^{3} r_{2} A_{i}^{(1)}-\kappa^{3} \sum_{j} g_{i j}\left|A_{j}^{(1)}\right|^{2} A_{i}^{(1)}-\kappa^{3} \sum_{j} f_{i j} A_{j}^{(1)} A_{j^{-}}^{(1)} \bar{A}_{i^{-}}^{(1)}-\kappa \alpha \sum_{j} h_{i j} A_{i}^{(1)}\left|B_{j}^{(1)}\right|^{2} \\
\kappa^{5} r_{2} \partial_{T} A_{i}^{(3)} & =\kappa^{5} r_{2} A_{i}^{(3)}-\cdots-\kappa^{5} \sum_{j} g_{i j}\left|A_{j}^{(1)}\right|^{2} \bar{A}_{i}^{(3)} \\
\kappa^{7} r_{2} \partial_{T} A_{i}^{(5)} & =\kappa^{7} r_{2} A_{i}^{(5)}-\cdots-\kappa^{7} \sum_{j} g_{i j}\left|A_{j}^{(3)}\right|^{2} A_{i}^{(1)}-\epsilon \kappa^{3} \sum_{j l k} h_{i j l k}\left|A^{(1)}\right|^{2}\left|B^{(1)}\right|^{2}\left|B^{(1)}\right|^{2} A_{i}^{(1)}
\end{aligned}
$$

which are solved order by order. To derive amplitude equations at seventh order we use the higher order coupling energies and neglect the low order coupling energies i.e. set $\alpha=0$. The higher order corrections to the uncoupled part are proportional to $\kappa^{5}$ and $\kappa^{7}$ while the inter-map coupling part is proportional to $\kappa^{3}$. For $\kappa \ll 1$ we thus can neglect fifth and seventh order corrections in the uncoupled dynamics.

We set $r_{2}=\tilde{r}_{2}=1$ and rescale the amplitudes as $A^{(1)}=r_{z}^{1 / 2} A^{(1)}, A^{(3)}=r_{z}^{3 / 2} A^{(3)}, A^{(5)}=$ $r_{z}^{5 / 2} A^{(5)}, B^{(1)}=r_{o}^{1 / 2} B^{(1)}, B^{(3)}=r_{o}^{3 / 2} B^{(3)}, B^{(5)}=r_{o}^{5 / 2} B^{(5)}$ and rescale to the fast time. This leads to

$$
\begin{aligned}
\partial_{t} A_{i}^{(1)} & =r_{z} A_{i}^{(1)}-\sum_{j} g_{i j}\left|A_{j}^{(1)}\right|^{2} A_{i}^{(1)}-\sum_{j} f_{i j} A_{j}^{(1)} A_{j^{-}}^{(1)} \bar{A}_{i^{-}}^{(1)} \\
\partial_{t} A_{i}^{(3)} & =r_{z} A_{i}^{(3)}-\cdots-\sum_{j} g_{i j}\left|A_{j}^{(1)}\right|^{2} \bar{A}_{i}^{(3)} \\
\partial_{t} A_{i}^{(5)} & =r_{z} A_{i}^{(5)}-\cdots-\sum_{j} g_{i j}\left|A_{j}^{(3)}\right|^{2} A_{i}^{(1)}-\epsilon \frac{1}{\kappa^{4}} \sum_{j l k} h_{i j l k}\left|A^{(1)}\right|^{2}\left|B^{(1)}\right|^{2}\left|B^{(1)}\right|^{2} A_{i}^{(1)}
\end{aligned}
$$


We can combine the amplitude equations up to seventh order by introducing the amplitudes $A_{j}=A_{j}^{(1)}+A_{j}^{(3)}+A_{j}^{(5)}$ and $B_{j}=B_{j}^{(1)}+B_{j}^{(3)}+B_{j}^{(5)}$. This leads to the amplitude equations

$$
\begin{aligned}
\partial_{t} A_{i}= & r_{z} A_{i}-\sum_{j} g_{i j}\left|A_{j}\right|^{2} A_{i}-\sum_{j} f_{i j} A_{j} A_{j^{-}} \bar{A}_{i^{-}} \\
& -\epsilon \frac{r_{z}^{2}}{r_{o}^{2} \kappa^{4}} \sum_{j l k} h_{i j l k}\left|A_{j}\right|^{2}\left|B_{l}^{2}\right|\left|B_{k}\right|^{2} A_{i} .
\end{aligned}
$$

Since $r_{z} / r_{o}=\kappa^{2}+\left(\kappa^{4} r_{4}-\kappa^{2} \tilde{r}_{4}\right) \mu^{2}+\ldots$ we finally obtain

$$
\begin{aligned}
\partial_{t} A_{i}= & r_{z} A_{i}-\sum_{j} g_{i j}\left|A_{j}\right|^{2} A_{i}-\sum_{j} f_{i j} A_{j} A_{j^{-}} \bar{A}_{i^{-}} \\
& -\epsilon \sum_{j l k} h_{i j l k}\left|A_{j}\right|^{2}\left|B_{l}^{2}\right|\left|B_{k}\right|^{2} A_{i} \\
\partial_{t} B_{i}= & r_{o} B_{i}-2 \bar{B}_{i+1} \bar{B}_{i+2}-\sum_{j} \tilde{g}_{i j}\left|B_{j}\right|^{2} A B_{i} \\
& -\epsilon \sum_{j l k} h_{i j l k}\left|B_{j}\right|^{2}\left|A_{l}^{2}\right|\left|A_{k}\right|^{2} B_{i} .
\end{aligned}
$$

In the following we use the amplitude equations (4.62) when considering the low order intermap coupling energies and the amplitude equations (4.67) when considering the higher order inter-map coupling energies.

\subsection{Inter-map coupling: Low order coupling terms}

In the following we discuss the influence of the OD stripe Eq. (4.29), hexagon Eq. (4.30) and constant Eq. (4.31) solutions on the OP map using the amplitude equations derived in the previous section. A potential backreaction onto the dynamics of the OD map can be neglected if the modes $A_{j}$ of the OP map are much smaller than the modes $B_{j}$ of the OD map. This can be achieved if $r_{z} \ll r_{o}$. We first give a brief description of the uncoupled OP solutions. Next, we study the impact of the low order coupling energies in Eq. (3.23) on these solutions. We demonstrate that these energies can lead to a complete suppression of orientation selectivity. In the uncoupled case there are for $r_{z}>0$ two stable stationary solutions to the amplitude equations Eq. (4.62), namely OP stripes

$$
z_{\mathrm{st}}=\mathcal{A} e^{\imath(k x+\phi)}, \quad \mathcal{A}=\sqrt{r_{z}},
$$

and $\mathrm{OP}$ rhombic solutions

$$
z_{\mathrm{rh}}=\mathcal{A} \sum_{j=1}^{2}\left(e^{\imath k_{j} x+\phi_{j}}+e^{-\imath k_{j} x+\phi_{j^{-}}}\right)
$$

with $\phi_{1}+\phi_{1^{-}}=\phi_{0}, \phi_{2}+\phi_{2^{-}}=\phi_{0}+\pi, \phi_{0}$ an arbitrary phase, and $\mathcal{A}=\sqrt{r_{z} / 5} \approx 0.447 \sqrt{r_{z}}$. In the uncoupled case the angle $\alpha=\arccos \overrightarrow{k_{1}} \overrightarrow{k_{2}} / k_{c}^{2}$ between the Fourier modes is arbitrary. The 
stripe solutions are pinwheel free while the pinwheel density for the rhombic solutions varies as $\rho=4 \sin \alpha$ and thus $0 \leq \rho \leq 4$. For the rhombic solutions pinwheels are located on a regular lattice. We therefore refer to these and other pinwheel rich solutions which are spatially periodic as pinwheel crystals (PWC). Without inter-map coupling, the potential of the two solutions reads $V_{\mathrm{st}}=-\frac{1}{2} r_{z}^{2}<V_{\mathrm{rh}}=-\frac{2}{5} r_{z}^{2}$, thus the stripe solutions are always energetically preferred compared to rhombic solutions. In the following we study three scenarios in which inter-map coupling can lead to pinwheel stabilization. First, a deformation of the stripe solution can lead to the creation of pinwheels in this solution. Second, inter-map coupling could energetically prefer the (deformed) rhombic solutions compared to the stripe solutions. Finally, inter-map coupling can lead to the stability of new PWC solutions.

For the low order interaction terms the amplitude equations are given by $\partial_{t} A_{i}=-\delta V / \delta \bar{A}_{i}$, $\partial_{t} B_{i}=-\delta V / \delta \bar{B}_{i}$ with the potential

$$
\begin{aligned}
V= & V_{A}+V_{B}+\sum_{j}^{3} \alpha \delta\left|A_{j}\right|^{2}+\sum_{j}^{3} \alpha \delta\left|A_{j-}\right|^{2} \\
& +2 \alpha \delta A_{1} \bar{A}_{2-} B_{3}+2 \alpha \delta A_{1} \bar{A}_{3-} B_{2}+2 \alpha \delta A_{1-} \bar{A}_{2} \bar{B}_{3}+2 \alpha \delta A_{1-} \bar{A}_{3} \bar{B}_{2} \\
& +\sum_{i, j} g_{i j}^{(1)}\left|A_{i}\right|^{2}\left|B_{j}\right|^{2}+\sum_{i \neq j} g_{i j}^{(2)} A_{i} \bar{A}_{j} \bar{B}_{i} B_{j}+\sum_{i \neq j} g_{i j}^{(2)} A_{i-} \bar{A}_{j-} B_{i} \bar{B}_{j} \\
& +\sum_{i, j} g_{i j}^{(3)} A_{i} \bar{A}_{j-} \bar{B}_{i} \bar{B}_{j}+\sum_{i, j} g_{i j}^{(3)} \bar{A}_{i} A_{j-} B_{i} B_{j} .
\end{aligned}
$$

The uncoupled contributions are given by

$$
\begin{aligned}
V_{A} & =-r_{z} \sum_{j}^{3}\left|A_{j}\right|^{2}+\frac{1}{2} \sum_{i, j}^{3} g_{i j}\left|A_{i}\right|^{2}\left|A_{j}\right|^{2}+\frac{1}{2} \sum_{i, j}^{3} f_{i j} A_{i} A_{i-} \bar{A}_{j} \bar{A}_{j^{-}} \\
V_{B} & =-r_{o} \sum_{j}^{3}\left|B_{j}\right|^{2}+\frac{1}{2} \sum_{i, j}^{3} \widetilde{g}_{i j}\left|B_{i}\right|^{2}\left|B_{j}\right|^{2} .
\end{aligned}
$$

The coupling coefficients read $g_{i j}^{(1)}=2 \alpha+2 \beta \cos ^{2}\left(\alpha_{i j}\right), g_{i j}^{(2)}=2 \alpha+\beta\left(1+\cos ^{2}\left(\alpha_{i j}\right)\right), g_{i j}^{(3)}=$ $2 \alpha+\beta\left(1+\cos ^{2}\left(\alpha_{i j}\right)\right), g_{i i}^{(3)}=\alpha+\beta$, where $\alpha_{i j}$ is the angle between the wavevector $\vec{k}_{i}$ and $\vec{k}_{j}$.

\subsubsection{Product-type energy $U=\alpha o^{2}|z|^{2}$}

We start with the low order product-type coupling energy. Here, the constant $\delta(\gamma)$ enters explicitly in the amplitude equations. We will see that this leads to a relatively complex phase diagram. 


\section{Stationary solutions and their stability}

In the case of OD stripes, Eq. (4.29), with $B_{1}=B_{\mathrm{st}}=B, B_{2}=B_{3}=0$ we get the following amplitude equations

$$
\begin{aligned}
& \partial_{t} A_{1}=\left(r_{z}-\alpha \delta^{2}-2 \alpha|B|^{2}\right) A_{1}-\alpha B^{2} A_{1^{-}}+\text {nct. } \\
& \partial_{t} A_{2}=\left(r_{z}-\alpha \delta^{2}-2 \alpha|B|^{2}\right) A_{2}-2 \alpha \delta A_{3^{-}} \bar{B}+\text { nct. } \\
& \partial_{t} A_{3}=\left(r_{z}-\alpha \delta^{2}-2 \alpha|B|^{2}\right) A_{3}-2 \alpha \delta A_{2^{-}} \bar{B}+\text { nct. }
\end{aligned}
$$

where nct. refers to non inter-map coupling terms resulting from the potential $V_{A}$, see Eq. (4.70). The equations for the modes $A_{i^{-}}$are given by interchanging $A_{i} \leftrightarrow A_{i^{-}}$and $B_{i} \leftrightarrow \bar{B}_{i}$. The OP stripe solution in case of inter-map coupling is given by

$$
z=\mathcal{A}_{1} e^{\imath\left(\vec{k}_{1} \vec{x}+\phi_{1}\right)}+\mathcal{A}_{1^{-}} e^{-\imath\left(\vec{k}_{1} \vec{x}+\phi_{1^{-}}\right)},
$$

with $\mathcal{A}_{1}=p^{3 / 2} /\left(2 \sqrt{2} B^{2} \alpha\right), \mathcal{A}_{1^{-}}=p / \sqrt{2}$, and $p=r_{z}-2 B^{2} \alpha-\alpha \delta^{2}-\sqrt{\left(r_{z}-\alpha \delta^{2}\right)\left(r_{z}-\alpha\left(4 B^{2}+\delta^{2}\right)\right)}$ and the phase relation $\phi_{1}-\phi_{1^{-}}=2 \psi_{1}+\pi$. In the uncoupled case $(\alpha=0) \mathcal{A}_{1^{-}}=0$ and $\mathcal{A}_{1}=\sqrt{r_{z}}$. With increasing inter-map coupling the amplitude $\mathcal{A}_{1-}$ grows and the solutions are transformed, reducing the representation of all but two preferred orientations. The course of this solution is shown in Fig. 4.3(a) for different values of the bias $\gamma$. Both amplitudes collapse at $\alpha=r_{z} /\left(4 B^{2}+\delta^{2}\right)$ with

$$
\mathcal{A}_{1}=\mathcal{A}_{1^{-}}=\sqrt{\frac{r_{z}-\alpha B^{2}-\alpha \delta^{2}}{3}}
$$

This pattern solution finally becomes unstable at

$$
\alpha_{c}=r_{z} /\left(B^{2}+\delta^{2}\right)=3 r_{z} / r_{o}
$$

This stability border is thus independent of the OD bias $\gamma$. Above this coupling strength only the trivial solution $A_{j}=0, \forall j$ is stable.

In addition to the OP stripe patterns there exist rhombic OP solutions, see Fig. 4.3(b). These rhombic solutions are pinwheel rich with a pinwheel density of $\rho=4 \sin \pi / 3 \approx 3.46$ but are energetically not preferred compared to the stripe solutions, see Fig. 4.3(e). The rhombic solutions in the uncoupled case $A_{1}=A_{1^{-}}=A_{2}=A_{2^{-}}, A_{3}=A_{3^{-}}=0$ are transformed by inter-map coupling. The phase relations are given by

$$
\begin{aligned}
\phi_{1}+\phi_{1^{-}} & =\phi_{0} \\
\phi_{2}+\phi_{2^{-}} & =\phi_{0}+\pi \\
\phi_{3}+\phi_{3^{-}} & =\phi_{0}+\pi \\
\phi_{1}-\phi_{1^{-}} & =2 \psi_{1} .
\end{aligned}
$$


Inter-map coupling reduces the amplitudes $A_{2}$ and $A_{2^{-}}$. In addition, for nonzero bias $\gamma$, there is an increase of the amplitudes $A_{3}=A_{3^{-}}$. The amplitudes $A_{2}$ and $A_{3}$ collapse. A further suppression of these amplitudes leads to the OP stripe pattern.

In the case the OD map is a constant, Eq. (4.31), the amplitude equations simplify to

$$
\partial_{t} A_{i}=\left(r_{z}-\alpha \delta^{2}\right) A_{i}-\sum_{j} g_{i j}\left|A_{j}\right|^{2} A_{i}-\sum_{j} f_{i j} A_{j} A_{j^{-}} \bar{A}_{i^{-}}
$$

Thus inter-map coupling in this case only renormalizes the control parameter and the stationary solution is thus a stripe pattern with a inter-map coupling dependent reduction of the amplitudes

$$
A_{i}=\sqrt{r_{z}-\alpha \delta^{2}}
$$

Therefore at $\alpha_{c}=r_{z} / \delta^{2}$ the stripe pattern becomes unstable and the only stable solution is the trivial one i.e. $A_{i}=0$.

In the case of OD hexagons $B_{i}=\mathcal{B} e^{\imath \psi_{i}}$, Eq. (4.30), the amplitude equations read

$$
\begin{aligned}
\partial_{t} A_{i}= & \left(r_{z}-6 \alpha \mathcal{B}^{2}-\alpha \delta^{2}\right) A_{i}-\alpha \mathcal{B}^{2}\left(A_{i^{-}} e^{2 \imath \psi_{i}}+2 A_{(i+1)}-e^{\imath\left(\psi_{i}+\psi_{i+1}\right)}+2 A_{(i+2)^{-}} e^{\imath\left(\psi_{i}+\psi_{i+2}\right)}\right) \\
& -2 \alpha \mathcal{B}^{2}\left(A_{i+1} e^{\imath\left(\psi_{i}-\psi_{i+1}\right)}+A_{i+2} e^{\imath\left(\psi_{i}-\psi_{i+2}\right)}\right) \\
& -2 \alpha \delta \mathcal{B}\left(A_{(i+1)^{-}} e^{-\imath \psi_{i+2}}+A_{(i+2)^{-}} e^{-\imath \psi_{i+1}}\right)+\text { nct. }
\end{aligned}
$$

where the indices are cyclic i.e. $i+3=i$. These amplitude equations have stripe-like solutions as well as solutions with a rhombic layout. The amplitudes in the rhombic case read $A_{1}=A_{1^{-}}=A_{3}=A_{3^{-}}, A_{2}=A_{2^{-}}$while the phase relations are given by $\phi=$ $(0, \varphi, 2 \varphi, \varphi+1,2 \varphi+1) \pi$. The phase $\varphi$ depends on the inter-map coupling strength $\alpha$.

In contrast to the case of OD stripes the amplitude equations have an additional type of PWC solution which have uniform amplitudes, $A_{j}=\mathcal{A} e^{\imath \phi_{i}}$. The dynamics of their phases is given by

$$
\begin{aligned}
\partial_{t} \phi_{i}= & 2 \mathcal{A}^{2} \sum_{j \neq i} \sin \left(\phi_{i}+\phi_{i^{-}}-\phi_{j}-\phi_{j^{-}}\right) \\
& -\mathcal{B}^{2} \alpha \sum_{j \neq i}\left(2 \sin \left(\phi_{i}-\phi_{j}-\psi_{i}+\psi_{j}\right)+2 \sin \left(\phi_{i}-\phi_{j^{-}}-\psi_{i}-\psi_{j}\right)\right) \\
& -\mathcal{B}^{2} \alpha \sin \left(\phi_{i}-\phi_{i^{-}}-2 \psi_{i}\right) \\
& -2 \delta \alpha \mathcal{B}\left(\sin \left(\phi_{i}-\phi_{(i+1)^{-}}+\psi_{i+2}\right)+\sin \left(\phi_{i}-\phi_{(i+2)^{-}}+\psi_{i+1}\right)\right)
\end{aligned}
$$

When solving the amplitude equations numerically we observe that the phase relations vary with the inter-map coupling strength for non-uniform solutions. But for the uniform solution the phase relations are independent of the inter-map coupling strength. The phases of the uniform solution are determined up to a free phase $\varphi$ which results from the orientation shift symmetry $z \rightarrow z e^{\imath \varphi}$ of Eq. (4.1). We therefore choose $\phi_{1}=\psi_{1}$. As an ansatz for the uniform 
solutions we use

$$
\begin{aligned}
\mathcal{A}_{j} & =\mathcal{A}_{j^{-}}=\mathcal{A}, \quad j=1,2,3 \\
\phi_{j} & =\psi_{j}+(j-1) 2 \pi / 3+\Delta \delta_{j, 2} \\
\phi_{j^{-}} & =-\psi_{j}+(j-1) 2 \pi / 3+\Delta\left(\delta_{j, 1}+\delta_{j, 3}\right),
\end{aligned}
$$

where $\delta_{i, j}$ is the Kronecker delta and $\Delta$ constant parameter. Note, that $z(\mathbf{x})$ cannot become real since $\phi_{j} \neq-\phi_{j^{-}}$. The equation for the uniform amplitudes is then given by

$$
\partial_{t} \mathcal{A}=r_{z} \mathcal{A}-9 \mathcal{A}^{3}-4 \alpha \mathcal{B}^{2} \mathcal{A}-\alpha \delta^{2} \mathcal{A}+\mathcal{A B} \alpha(\mathcal{B}-2 \delta) \cos \Delta
$$

while the phase dynamics reads

$$
\partial_{t} \phi_{j}=-\mathcal{B} \alpha(\mathcal{B}-2 \delta) \sin \Delta
$$

The stationarity condition is fulfilled for an arbitrary $\delta$ only if $\Delta=0$ or $\Delta=\pi$. The corresponding amplitudes are given by solving the stationarity condition for the real part and read

$$
\mathcal{A}_{\Delta=0}=\sqrt{\frac{r_{z}-\alpha\left(3 \mathcal{B}^{2}+2 \mathcal{B} \delta+\delta^{2}\right)}{9}}, \quad \mathcal{A}_{\Delta=\pi}=\sqrt{\frac{r_{z}-\alpha\left(5 \mathcal{B}^{2}-2 \mathcal{B} \delta+\delta^{2}\right)}{9}} .
$$

The stability of the $\Delta=0, \pi$ solutions depends on the coupling strength $\alpha$ and on the sign of $(\mathcal{B}-2 \delta)$. As the solution of $B(\gamma)=2 \delta(\gamma)$ is given by $\gamma=\gamma^{*}$ in the stability range of OD hexagons there is only one possible stable uniform solution. The $\Delta=\pi$ solution becomes unstable at $r_{z}<\alpha\left(5 \mathcal{B}^{2}-2 \mathcal{B} \delta+\delta^{2}\right)$. This stability border is in fact independent of the bias $\gamma$ and given by

$$
\alpha_{c}=3 r_{z} / r_{o} .
$$

Thus the limit $r_{z} \rightarrow 0$ makes the uniform solution unstable for smaller and smaller coupling strengths.

We calculate the stability properties of the uniform solution by linear stability analysis considering perturbations of the amplitudes $\mathcal{A}_{j} \rightarrow \mathcal{A}+a_{j}, \mathcal{A}_{j^{-}} \rightarrow \mathcal{A}+a_{j^{-}}$and of the phases $\phi_{j} \rightarrow \phi_{j}+\varphi_{j}, \phi_{j^{-}} \rightarrow \phi_{j^{-}}+\varphi_{j^{-}}$. This leads to a perturbation matrix $M$. Amplitude and phase perturbations do not decouple. We calculate the eigenvalues of the perturbation $M$ matrix numerically.

\section{Bifurcation diagram}

The course of OP solutions when interacting with OD stripes is shown in Fig. 4.3(a,b). In case of OP stripes inter-map coupling suppresses the amplitude $A_{1}$ of the stripe pattern while increasing the amplitude of the opposite mode $A_{1^{-}}$. This transformation reduces the representation of all but two preferred orientations. When both amplitudes collapse the resulting OP map is selective only to two orthogonal orientations namely $\vartheta=\phi_{1}$ and $\vartheta=\phi_{1}+\pi / 2$. 
We refer to these unrealistic solutions as orientation scotoma solutions. The phase relations ensure that OD borders that run parallel to the OP stripes are located at the OP maxima and minima i.e. in the center of the orientation scotoma stripes. With increasing intermap coupling, this orientation scotoma pattern is suppressed until finally all amplitudes are zero and only the homogeneous solution is stable. In case of OP rhombs inter-map coupling makes the rhombic pattern more stripe-like by reducing the amplitude $A_{2}=A_{2^{-}}$. The mode $A_{3}=A_{3^{-}}$which is zero in the uncoupled case increases and finally collapses with the mode $A_{2}$. Increasing inter-map coupling more suppresses all but the two modes $A_{1}=A_{1}^{-}$, leading again to the orientation scotoma stripe pattern.

The course of OP solutions when interacting with OD hexagons is shown in Fig. 4.3(c,d). OP stripe solutions become above a critical inter-map coupling strength unstable against PWC solutions. This critical coupling strength strongly depends on the OD bias. OP rhombic solutions also become unstable against PWC but for a lower coupling strength than the OP stripes. Thus there is at intermediate coupling strength a bistability between stripe-like solutions and PWC solutions. The potential of the OP stripe and OP rhombic solutions is shown in Fig. 4.3 (e,f). Stripes are energetically preferred in the uncoupled case as well as for small inter-map coupling strength for which they are stable.

To summarize, stripe solutions are deformed but no pinwheels are created for this solution. The rhombic solutions are for low inter-map coupling energetically not preferred whereas for intermediate inter-map coupling these solutions lose pinwheels and become stripe solutions. Instead, additional pinwheel rich solutions with a crystal layout become stable for intermediate inter-map coupling. For large inter-map coupling orientation selectivity is completely suppressed. 

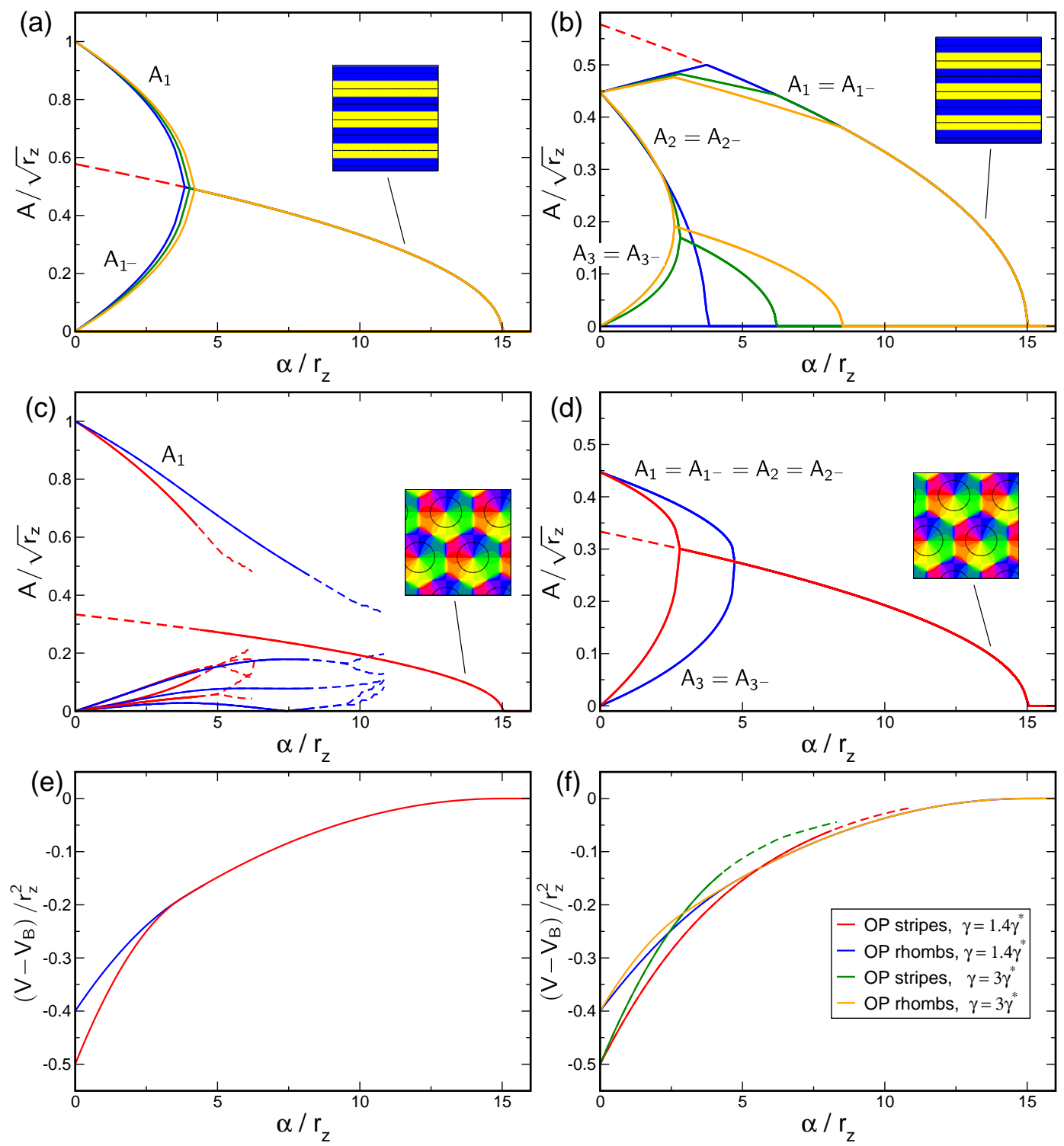

Figure 4.3.: Stationary amplitudes with coupling energy $\mathbf{U}=\alpha|\mathbf{z}|^{2} \mathbf{o}^{2}, r_{o}=0.2$. Solid (dashed) lines: stable (unstable) solutions to Eq. (4.72) (OD stripes) and Eq. (4.79) (OD hexagons). (a,b) OD stripes, $\gamma=0$ (blue), $\gamma=\gamma^{*}$ (green), $\gamma=1.4 \gamma^{*}$ (orange). (c,d) OD hexagons, $\gamma=1.4 \gamma^{*}$ (blue), $\gamma=3 \gamma^{*}$ (red). (e) Potential for OP stripes (red) and OP rhombs (blue) interacting with OD stripes, $\gamma=0$. (f) Potential for OP stripes and OP rhombs interacting with OD hexagons. 


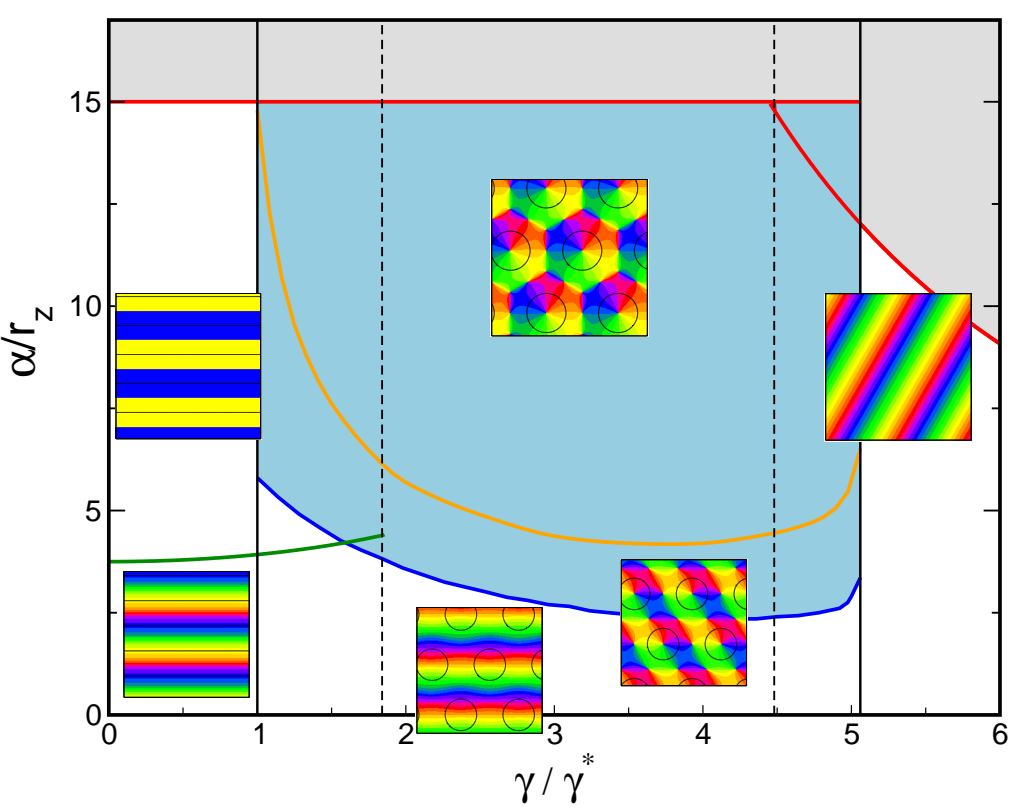

Figure 4.4.: Phase diagram with the coupling energy $\mathbf{U}=\alpha \mathbf{o}^{\mathbf{2}}|\mathbf{z}|^{2}, r_{o}=0.2, r_{z} \ll r_{o}$. Vertical lines: stability range of OD stripes, hexagons, and constant solution. Green line: transition to the orientation scotoma solution. Blue line: stability border for the $\Delta=\pi$ uniform solution. Orange line: stability line of stripe-like solutions. Red line: pattern solutions become unstable, see Eq. (4.75) and Eq. (4.85). Gray region: No pattern solution stable.

\section{Phase diagram}

The phase diagram for this coupling energy is shown in Fig. 4.4. The phase diagram contains the stability borders of the uncoupled OD solutions $\gamma^{*}, \gamma_{2}^{*}, \gamma_{3}^{*}, \gamma_{4}^{*}$. They correspond to vertical lines, as they are independent of the inter-map coupling in the limit $r_{z} \ll r_{o}$. At $\gamma=\gamma^{*}$ hexagons become stable. Stripe solutions become unstable at $\gamma=\gamma_{2}^{*}$. At $\gamma=\gamma_{3}^{*}$ the homogeneous solution becomes stable while at $\gamma=\gamma_{4}^{*}$ hexagons lose their stability. In the units $\gamma / \gamma^{*}$ the borders $\gamma_{2}^{*}, \gamma_{3}^{*}, \gamma_{4}^{*}$ vary slightly with $r_{o}$, see Fig. 4.2, and are drawn here for $r_{o}=0.2$. The inter-map coupling strength $\alpha$ is rescaled by $r_{z}$ and thus the stability borders are independent of $r_{z}$. In the region of stable OD stripes the OP stripes run parallel to the OD stripes. With increasing inter-map coupling strength the orientation preference of all but two orthogonal orientations is suppressed. In the region of stable OD hexagons stripe-like OP solutions dominate for low inter-map coupling strength. Above a critical bias dependent coupling strength the $\Delta=\pi$ uniform solution becomes stable (blue line). There is a region of bistability between stripe-like and uniform solutions until the stripe-like solutions lose their stability (orange line). OP rhombic solutions lose their stability when the uniform solution becomes stable. Thus there is no bistability between OP rhombs and OP uniform solutions. As in the case of OD stripes the uniform solution becomes unstable at $\alpha=r_{z} /\left(3 \mathcal{B}^{2}\right)$. Also in the case of OD hexagons the inter-map coupling leads to a transition towards the trivial solution where there is no OP pattern at all. In case of the OD constant solution the OP 
map is a stripe solution. Pinwheel rich solutions thus occur only in the region of stable OD hexagons. In the following we discuss the properties of these solutions.

\section{Pinwheel crystals}

The uniform solution Eq. (4.81) with $\Delta=\pi$ is illustrated in Fig. 4.5, For all stationary

(a)

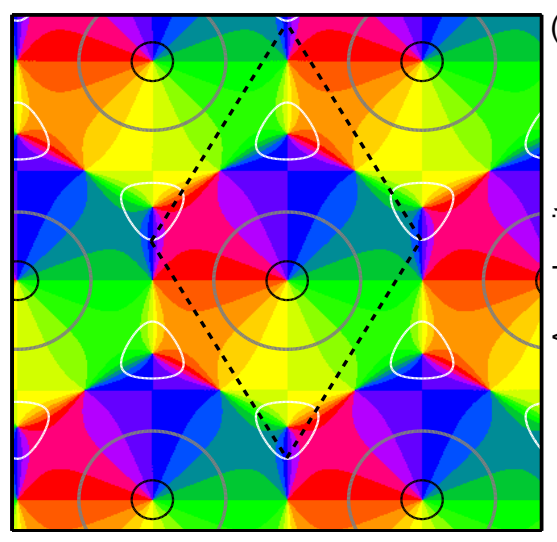

(b)

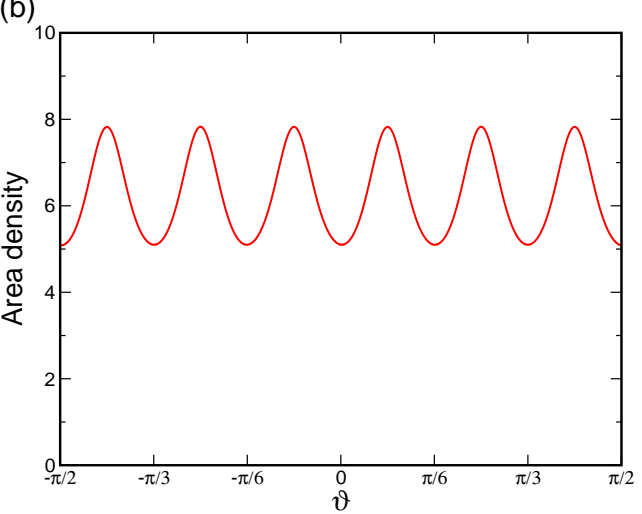

Figure 4.5.: Ipsi-center pinwheel crystal. (a) OP map, superimposed are the OD borders (gray), 90\% ipsilateral eye dominance (black), and $90 \%$ contralateral eye dominance (white), $r_{o}=0.2, \gamma=3 \gamma^{*}$. Dashed lines mark the unit cell of the regular pattern. (b) Distribution of preferred orientations.

solutions the positions of the pinwheels are fixed by the OD map and there are no translational degrees of freedom. The unit cell (dashed line) contains 6 pinwheels which leads to a pinwheel density of $\rho=6 \cos \pi / 6 \approx 5.2$. Two of them are located at OD maxima (contra center) while one is located at an OD minimum (ipsi center). The remaining three pinwheels are located at OD saddle-points. Therefore, all pinwheels are located where the gradient of the OD map is zero. The pinwheel in the center of the OP hexagon is at the ipsilateral OD peak. Because these pinwheels organize most of the map while the others essentially only match one OP hexagon to its neighbors we refer to this pinwheel crystal as the Ipsi-center pinwheel crystal. The iso-orientation lines intersect the OD borders (gray) exactly with a right angle. The intersection angles are, within the stability range of OD hexagons, independent of the bias $\gamma$. The remarkable property of perfect intersection angles cannot be deduced directly from the coupling energy term. We expect that this is a property of the crystal structure that is altered in spatially aperiodic OP maps. The solution is symmetric under a combined rotation by $60^{\circ}$ and an orientation shift by $-60^{\circ}$. The symmetry of the pattern is reflected by the distribution of preferred orientations, see Fig. 4.5(b). Although the pattern is selective to all orientations the six orientations $\vartheta+n \frac{\pi}{6}, n=0, \ldots, 5$ are slightly overrepresented.

To summarize, the low order product-type inter-map coupling leads in case of OD hexagons to a transition from pinwheel free stripe solutions towards pinwheel crystals. The design of the PWC is an example of an orientation hypercolumn dominated by one pinwheel. With increasing inter-map coupling the crystal solution is suppressed until only the homogeneous 
solution is stable. In case of OD stripes or the constant solution the OP solutions are pinwheel free stripe pattern.

\subsubsection{Gradient-type energy $U=\beta|\nabla o \nabla z|^{2}$}

When using a gradient-type inter-map coupling energy the interaction terms are independent of the OD shift $\delta$. In this case the coupling strength can be rescaled as $\beta \mathcal{B}^{2}$ and is therefore independent of the bias $\gamma$. The bias in this case only determines the stability of OD stripes, hexagons or the constant solution.

\section{Stationary solutions and their stability}

A coupling to OD stripes is easy to analyze in the case of a gradient-type inter-map coupling. The energetically preferred solutions are OP stripes with the direction perpendicular to the OD stripes for which $U=0$. This configuration corresponds to the Hubel and Wiesel Ice-cube model [26].

In the case the OD map is a constant, Eq. (4.31), the gradient-type inter-map coupling leaves the OP unaffected. The stationary states are therefore OP stripes with an arbitrary direction. In the case of OD hexagons the amplitude equations read

$$
\begin{aligned}
\partial_{t} A_{i}= & \left(r_{z}-3 \beta \mathcal{B}^{2}\right) A_{i}+\frac{5}{4} \beta \mathcal{B}^{2}\left(A_{i+1} e^{\imath\left(\psi_{i}-\psi_{i+1}\right)}+A_{i+2} e^{\imath\left(\psi_{i}-\psi_{i+2}\right)}\right) \\
& -\beta \mathcal{B}^{2}\left(A_{i^{-}} e^{2 \imath \psi_{i}}+\frac{5}{4} A_{(i+1)^{-}} e^{\imath\left(\psi_{i}+\psi_{i+1}\right)}+\frac{5}{4} A_{(i+2)^{-}} e^{\imath\left(\psi_{i}+\psi_{i+2}\right)}\right)+\text { nct. }
\end{aligned}
$$

Using $A_{i}=\mathcal{A}_{i} e^{\imath \phi_{i}}$ we obtain the phase equations

$$
\begin{aligned}
\mathcal{A}_{i} \partial_{t} \phi_{i}= & \sum_{j \neq i} \mathcal{A}_{j} \mathcal{A}_{j^{-}} \mathcal{A}_{i^{-}} \sin \left(\phi_{i}+\phi_{i^{-}}-\phi_{j}-\phi_{j^{-}}\right) \\
& -\mathcal{B}^{2} \beta \sum_{j \neq i}\left(\frac{5}{4} \mathcal{A}_{j} \sin \left(\phi_{i}-\phi_{j}-\psi_{i}+\psi_{j}\right)+\frac{5}{4} \mathcal{A}_{j^{-}} \sin \left(\phi_{i}-\phi_{j^{-}}-\psi_{i}-\psi_{j}\right)\right) \\
& -\mathcal{B}^{2} \beta \mathcal{A}_{i^{-}} \sin \left(\phi_{i}-\phi_{i^{-}}-2 \psi_{i}\right)
\end{aligned}
$$

Besides stripe-like and rhombic solutions these amplitude equations have uniform solutions. Again we find that the ansatz Eq. (4.81) can satisfy the stationarity condition. The phase dynamics in this case reads

$$
\partial_{t} \phi_{i}=-\frac{1}{4} \mathcal{B}^{2} \beta \sin \Delta
$$

As in the case of the product-type inter-map coupling energy stationary solutions are $\Delta=0$ and $\Delta=\pi$ with the stationary amplitudes

$$
\mathcal{A}_{\Delta=0}=\sqrt{\frac{r_{z}-3 / 2 \mathcal{B}^{2} \beta}{9}}, \quad \mathcal{A}_{\Delta=\pi}=\sqrt{\frac{r_{z}-2 \mathcal{B}^{2} \beta}{9}} .
$$

We study the stability properties of both stationary solutions by linear stability analysis where amplitude and phase perturbations in general do not decouple. It turns out that the $\Delta=\pi$ 


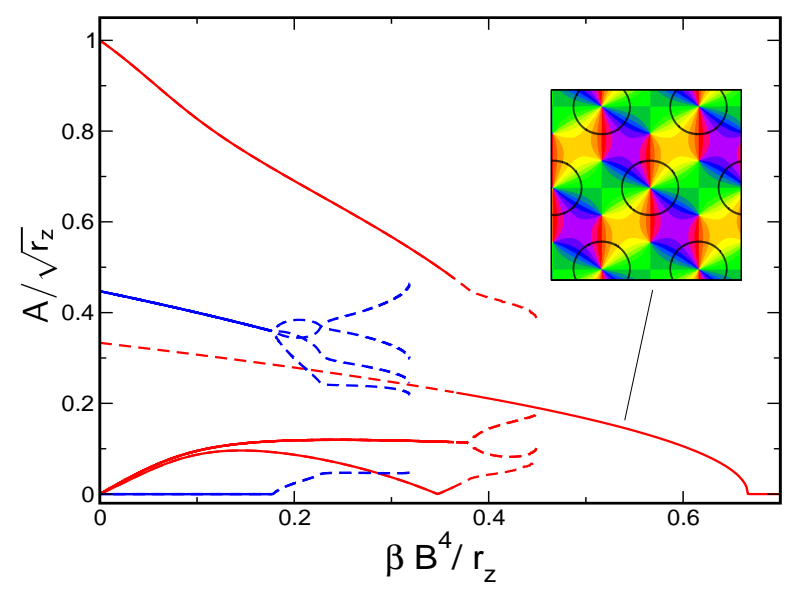

Figure 4.6: Stationary amplitudes with $\mathbf{U}=\beta|\nabla \mathbf{z} \nabla \mathbf{o}|^{2}$ and OD hexagons. Solid (dashed) lines: Stable (unstable) solutions to Eq. (4.86). Transition from OP stripes towards the uniform solution (red), transition from OP rhombs towards the uniform solution (blue).

solution is unstable for $\beta>0$ while the $\Delta=0$ solution becomes stable for $\beta \approx 0.05 r_{z} / \mathcal{B}^{2}$. The $\Delta=0$ solution loses its stability above

$$
\beta_{c} \mathcal{B}^{2}=2 r_{z} / 3
$$

From thereon only the homogeneous solution $A_{j}=0$ is stable.

\section{Bifurcation diagram}

The course of the stationary amplitudes when interacting with OD hexagons is shown in Fig. 4.6. The OP rhombic solution is almost unchanged by inter-map coupling but above a critical coupling strength the rhombs decay into a stripe-like solution. The amplitude of the OP stripe solution is suppressed by inter-map coupling and finally becomes unstable against the $\Delta=0$ uniform solution. Thus for large inter-map coupling only the uniform solution is stable. A further increase in the inter-map coupling suppresses the amplitude of this uniform solution until finally only the homogeneous solution is stable.

\section{Phase diagram}

The phase diagram of this coupling energy is shown in Fig. 4.7. We rescale the inter-map coupling strength as $\beta \mathcal{B}^{2} / r_{z}$, where $\mathcal{B}$ is the stationary amplitude of the OD hexagons. The stability borders are therefore independent of the OD bias in the OD solutions. This simplifies the analysis a lot since the OP solutions and their stability depend on $\gamma$ only indirect via the amplitudes $B$. In case of OD stripes or OD constant solution there is no pinwheel crystallization. Instead the OP solutions are pinwheel free stripes. In case of OD hexagons hexagonal pinwheel crystals become stable above $\beta \approx 0.05 r_{z} / \mathcal{B}^{2}$ (blue line). Rhombic OP patterns become unstable at $\beta \mathcal{B}^{4} / r_{z} \approx 0.17$ and decay into a stripe-like solution (green line). At $\beta \mathcal{B}^{4} / r_{z} \approx 0.36$ these stripe-like solutions become unstable (orange line). Thus above $\beta \mathcal{B}^{4} / r_{z} \approx 0.36$ the hexagonal PWC is the only stable solution. At $\beta \mathcal{B}^{2} / r_{z}=2 / 3$ the pattern solution is unstable (red line). 


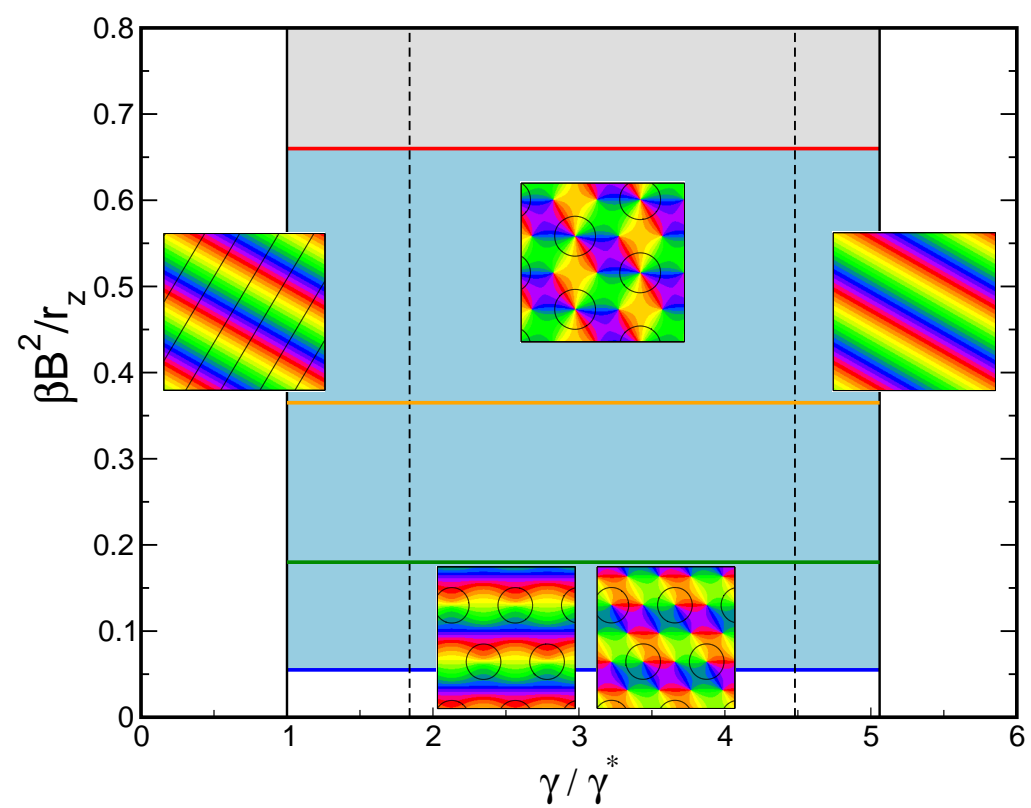

Figure 4.7.: Phase diagram with the coupling energy $\mathbf{U}=\beta|\nabla \mathbf{z} \nabla \mathbf{o}|^{2}, r_{z} \ll r_{o}$. Vertical black lines: stability range of OD stripes, hexagons, and constant solution. Blue line: stability border for the $\Delta=0$ uniform solution. Green line: rhombic solutions become unstable. Orange line: stripe-like solutions become unstable. Red line: pattern solutions become unstable, see Eq. (4.89). Gray region: No pattern solution stable.

\section{Pinwheel crystals}

The uniform solution Eq. (4.81), $\Delta=0$ is illustrated in Fig. 4.8, This PWC contains only three pinwheels per unit cell leading to a pinwheel density of $\rho=3 \cos \pi / 6 \approx 2.6$. Two of the three pinwheels are located at maxima of the OD map (contra peak) while the remaining pinwheel is located at the minimum (ipsi peak) of the OD map. A remarkable property of this solution is that the pinwheel located at the OD minimum, carries a topological charge of 1 such that each orientation is represented twice around this pinwheel. Pinwheels of this kind have not yet been observed in physiological OP maps. This kind of uniform solution corresponds to the structural pinwheel model by Braitenberg [98. We therefore refer to this solution as the Braitenberg pinwheel crystal.

The iso-orientation lines are again perfectly perpendicular to OD borders and also independent of the bias $\gamma$. The solution is symmetric under a combined rotation by $120^{\circ}$ and an orientation shift by $-2 \pi / 3$. Further it is symmetric under a rotation by $180^{\circ}$. The pattern is selective to all orientations but the distribution of represented orientations is not flat. The three orientations $\vartheta+n \frac{\pi}{3}, n=0, \ldots, 2$ are overrepresented, see Fig. 4.8(b).

Overall this OP map is dominated by uniform regions around hyperbolic points. In contrast to the ipsi center PWC all pinwheels in this OP map organize a roughly similar fraction of the cortical surface. 
(a)

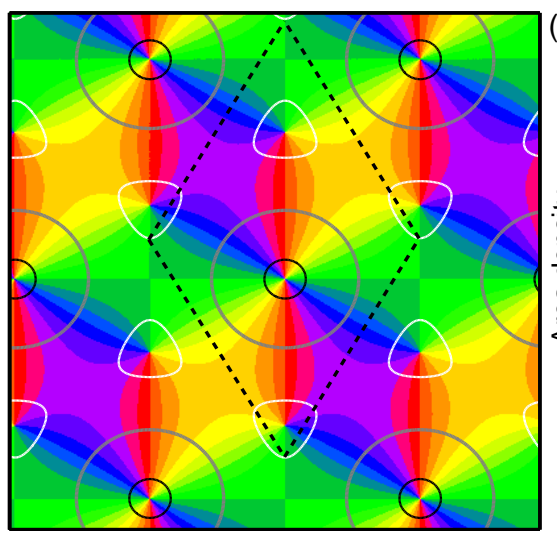

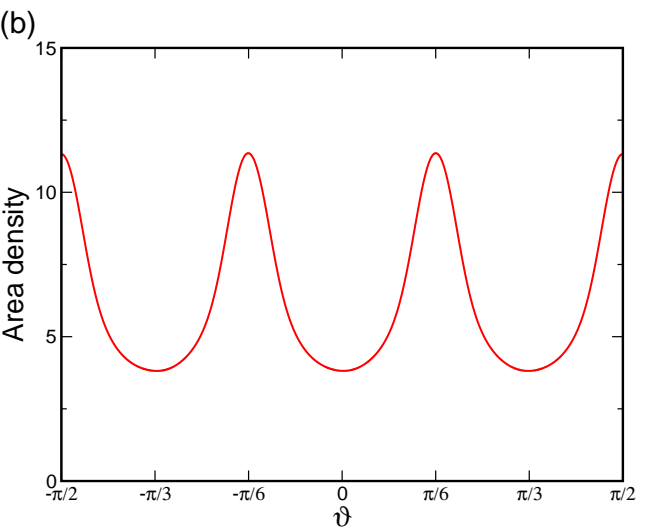

Figure 4.8.: The Braitenberg pinwheel crystal, $\Delta=0$ uniform solution of Eq. (4.81). (a) OP map, superimposed are the OD borders (gray), 90\% ipsilateral eye dominance (black), and $90 \%$ contralateral eye dominance (white), $r_{o}=0.2, \gamma=3 \gamma^{*}$. Dashed lines mark the unit cell of the regular pattern. (b) Distribution of preferred orientations.

\subsection{Inter-map coupling: Higher order coupling terms}

In the last section we demonstrated that the low order coupling terms can lead to a complete suppression of OP selectivity. As the coupling terms are effectively linear they not only influence pattern selection but also whether there is a pattern at all. In this section we therefore study the impact of higher order coupling energies using the amplitude equations Eq. (4.67). In this case the effective coupling is a cubic interaction term and complete selectivity suppression is impossible. Moreover, we can identify the limit $r_{z} \ll r_{o}$ in which the backreaction onto the OD map can be neglected. When using the higher order inter-map coupling terms and assuming $B_{i}=\mathcal{B} e^{\imath \psi_{i}}$ the potential reads

$$
\begin{aligned}
V= & V_{A}+V_{B}+ \\
& +\epsilon \mathcal{B}^{4} \sum_{i j l k}^{3}\left(h_{i j l k}^{(1)} A_{j} A_{l} \bar{A}_{k} \bar{A}_{i}+h_{i j l k}^{(2)} A_{j^{-}} A_{l^{-}} \bar{A}_{k^{-}} \bar{A}_{i}+h_{i j l k}^{(3)} A_{j} A_{l^{-}} \bar{A}_{k} \bar{A}_{i}\right. \\
& +h_{i j l k}^{(4)} A_{j^{-}} A_{l^{-}} \bar{A}_{k} \bar{A}_{i}+h_{i j l k}^{(5)} A_{j} A_{l} \bar{A}_{k^{-}} \bar{A}_{i}+h_{i j l k}^{(6)} A_{j} A_{l^{-}} \bar{A}_{k^{-}} \bar{A}_{i} \\
& \left.+\tilde{h}_{i j l k}^{(1)} A_{j^{-}} A_{l^{-}} \bar{A}_{k^{-}} \bar{A}_{i^{-}}+\tilde{h}_{i j l k}^{(3)} A_{j^{-}} A_{l} \bar{A}_{k^{-}} \bar{A}_{i^{-}}+\tilde{h}_{i j l k}^{(4)} A_{j} A_{l} \bar{A}_{k^{-}} \bar{A}_{i^{-}}\right)
\end{aligned}
$$

where $\tilde{h}_{i j l k}^{(1)}=h_{i j l k ; \psi_{j} \rightarrow-\psi_{j}}^{(1)}, \tilde{h}_{i j l k}^{(3)}=h_{i j l k ; \psi_{j} \rightarrow-\psi_{j}}^{(3)}, \tilde{h}_{i j l k}^{(4)}=h_{i j l k ; \psi_{j} \rightarrow-\psi_{j}}^{(4)}$. The amplitude equations can be derived from a potential given by

$$
\begin{aligned}
\partial_{t} A_{i}= & r_{z} A_{i}-\sum_{j}^{3} g_{i j}\left|A_{j}\right|^{2} A_{i}-\sum_{j \neq i}^{3} f_{i j} A_{j} A_{j^{-}} \bar{A}_{i^{-}} \\
& -\mathcal{B}^{4} \sum_{j, l, k}^{3}\left(h_{i j l k}^{(1)} A_{j} A_{l} \bar{A}_{k}+h_{i j l k}^{(2)} A_{j^{-}} A_{l^{-}} \bar{A}_{k^{-}}+h_{i j l k}^{(3)} A_{j} A_{l^{-}} \bar{A}_{k}\right. \\
& \left.+h_{i j l k}^{(4)} A_{j^{-}} A_{l^{-}} \bar{A}_{k}+h_{i j l k}^{(5)} A_{j} A_{l} \bar{A}_{k^{-}}+h_{i j l k}^{(6)} A_{j} A_{l^{-}} \bar{A}_{k^{-}}\right),
\end{aligned}
$$


with $g_{i i}=1, g_{i j}=2$ and $h_{i j l k}^{(u)}$ effective self-interaction coupling coefficients given in Appendix A.2. We have not written terms containing the constant $\delta$ for simplicity. The phases $\psi_{j}$ are absorbed into the coupling coefficients. The dynamics for the modes $A_{i^{-}}$is given by interchanging $A_{i}$ and $A_{i^{-}}$. For negligible backreaction $\mathcal{B}=\mathcal{B}_{\text {hex }}, \mathcal{B}=\mathcal{B}_{\text {st }}$, or $\mathcal{B}=\mathcal{B}_{c}$. In the following we identify classes of stationary solutions of the amplitude equations Eq. (4.92) and provide their stability criteria for the two higher order pendants of the coupling energies.

\subsubsection{Product-type energy $U=\tau o^{4}|z|^{4}$}

First, we study the higher order product-type inter-map coupling energy in Eq. (3.23). As for the lower order version of this coupling energy the shift $\delta(\gamma)$ explicitly enters the amplitude equations resulting in a rather complex phase diagram.

\section{Stationary solutions and their stability}

In the case of OD stripes the amplitude equations of OP modes read

$$
\begin{aligned}
& \partial_{t} A_{1}=r_{z} A_{1}-\sum_{j}\left(g_{1 j}^{(1)}\left|A_{j}\right|^{2} A_{1}+g_{1 j}^{(2)}\left|A_{j}\right|^{2} A_{1^{-}}+g_{1 j}^{(3)} A_{j} A_{j^{-}} \bar{A}_{1^{-}}+g_{1 j}^{(4)} A_{j} A_{j^{-}} \bar{A}_{1}\right) \\
& -B^{4} A_{1^{-}}^{2} \bar{A}_{1}-\sum_{u \neq v \neq w} A_{u} A_{v} \bar{A}_{w}\left(\left(8 \delta^{3} B+24 \delta B^{2} \bar{B}\right) \delta_{\vec{k}_{u}+\vec{k}_{v}-\vec{k}_{w}, 0}\right. \\
& \left.+\left(8 \delta^{3} \bar{B}+24 \delta B \bar{B}^{2}\right) \delta_{\vec{k}_{u}+\vec{k}_{v}-\vec{k}_{w}, 2 \vec{k}_{1}}+8 \delta B^{3} \delta_{\vec{k}_{u}+\vec{k}_{v}-\vec{k}_{w},-2 \vec{k}_{1}}\right) \\
& \partial_{t} A_{2}=r_{z} A_{2}-\sum_{j}\left(g_{2 j}^{(1)}\left|A_{j}\right|^{2} A_{2}+g_{2 j}^{(3)} A_{j} A_{j^{-}} \bar{A}_{2^{-}}\right) \\
& -g_{i i}^{(2)} A_{2} A_{1^{-}} \bar{A}_{1}-g^{(5)} A_{2} A_{1} \bar{A}_{1^{-}}-1 / 2 g_{i i}^{(2)} A_{1^{-}}^{2} \bar{A}_{2^{-}}-1 / 2 g^{(5)} A_{1}^{2} \bar{A}_{2^{-}} \\
& -\sum_{u, v, w} A_{u} A_{v} \bar{A}_{w}\left(g_{u v}^{(6)} \delta_{\vec{k}_{u}+\vec{k}_{v}-\vec{k}_{w}, \vec{k}_{2}}+g_{i j}^{(7)} \delta_{\vec{k}_{u}+\vec{k}_{v}-\vec{k}_{w}, \vec{k}_{1}+\vec{k}_{2}}\right.
\end{aligned}
$$

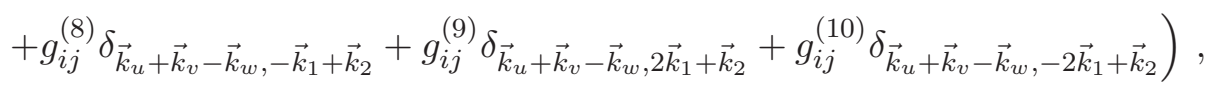

where $\delta_{i, j}$ denotes the Kronecker delta and $g_{i i}^{(1)}=1+\delta^{4}+12 \delta^{2}|B|^{2}+6|B|^{4}, g_{i j}^{(1)}=2 g_{i i}^{(1)}, g_{i i}^{(2)}=$ $g_{i j}^{(2)}=12 \delta^{2} B^{2}+8 B^{3} \bar{B}, g_{i j}^{(3)}=2+12|B|^{4}+24 \delta^{2}|B|^{2}+2 \delta^{4}, g_{i i}^{(3)}=0, g_{i j}^{(4)}=12 \delta^{2} B^{2}+8 B^{3} \bar{B}$, $g_{i i}^{(4)}=0, g^{(5)}=12 \delta^{2} \bar{B}^{2}+8 B \bar{B}^{3}, g_{u u}^{(6)}=6|B|^{4}+6 \delta|B|^{2}, g_{u v}^{(6)}=2 g_{u u}^{(6)}, g_{u u}^{(7)}=4 \bar{B} \delta^{3}+1 B \bar{B}^{2} \delta$, $g_{u v}^{(7)}=2 g_{u u}^{(7)}, g_{u u}^{(8)}=4 B \delta^{3}+1 B^{2} \bar{B} \delta, g_{u v}^{(8)}=2 g_{u u}^{(8)}, g_{u u}^{(9)}=6 \bar{B}^{2} \delta^{2}, g_{u v}^{(9)}=2 g_{u u}^{(9)}, g_{u u}^{(10)}=6 B^{2} \delta^{2}$, $g_{u v}^{(10)}=2 g_{u u}^{(10)}$. The equation for the mode $A_{3}$ is given by interchanging $A_{2}$ and $A_{3}$ in Eq. (4.93). The equations for the modes $A_{i^{-}}$are given by interchanging $A_{i} \leftrightarrow A_{i^{-}}$and $B_{i} \leftrightarrow \bar{B}_{i}$.

In this case, at low inter-map coupling the OP stripes given by

$$
z=\mathcal{A}_{1} e^{\imath\left(\vec{k}_{1} \vec{x}+\phi_{1}\right)}-\mathcal{A}_{1^{-}} e^{-\imath\left(\vec{k}_{1} \vec{x}+\phi_{1^{-}}\right)},
$$

with $\phi_{1}-\phi_{1^{-}}=2 \psi_{1}+\pi$ run parallel to the OD stripes. The course of these stripe solutions is shown in Fig. 4.9(a).

At large inter-map coupling the attractor states of the OP map consist of a stripe pattern 
containing only two preferred orientations, namely $\vartheta=\phi_{1}$ and $\vartheta=\phi_{1}+\pi / 2$. The zero contour lines of the OD map are along the maximum amplitude of orientation preference minimizing the energy term.

In addition there are rhombic solutions

$$
z=\mathcal{A}_{1} e^{\imath\left(\vec{k}_{1} \vec{x}+\psi_{1}\right)}+\mathcal{A}_{1^{-}} e^{-\imath\left(\vec{k}_{1} \vec{x}-\psi_{1}+\pi\right)}+\mathcal{A}_{2} e^{\imath\left(\vec{k}_{2} \vec{x}+\psi_{1}\right)}+\mathcal{A}_{2^{-}} e^{-\imath\left(\vec{k}_{2} \vec{x}-\psi_{1}\right)},
$$

which exist also in the uncoupled case, see Fig. 4.9(b). However, these rhombic solutions are energetically not favored compared to stripe solutions, see Fig. 4.9(c). The inclusion of the inter-map coupling makes these rhombic solution even more stripe-like. In case of a OD constant solution the amplitude equations read

$$
\partial_{t} A_{i}=r_{z} A_{i}-\sum_{j} g_{i j}\left|A_{j}\right|^{2} A_{i}-\sum_{j} f_{i j} A_{j} A_{j^{-}} \bar{A}_{i^{-}}
$$

with $g_{i i}=1+\delta^{4} \tau, g_{i j}=2+2 \delta^{4} \tau$ and $f_{i j}=2+2 \delta \tau$. Inter-map coupling thus only leads to a renormalization of the uncoupled interaction terms. Stationary solutions are therefore stripes with the amplitude

$$
\mathcal{A}=\sqrt{\frac{r_{z}}{1+\delta^{4} \tau}}
$$

Next, we study the impact of OD hexagons on the dynamics of OP maps. In addition to stripe-like and rhombic solutions we find uniform solutions. When solving the amplitude equations numerically we have seen that the phase relations vary with the inter-map coupling strength $\tau$ for non-uniform solutions. But for the uniform solution the phase relations are independent of the inter-map coupling strength. When we use the ansatz Eq. (4.81) for uniform solutions we get the stationarity condition

$$
6 \mathcal{A}^{2} \mathcal{B}\left[4\left(-4 B^{3}+7 B^{2} \delta-B \delta^{2}+\delta^{3}\right)+\mathcal{B} \cos \Delta\left(13 B^{2}-8 B \delta+6 \delta^{2}\right)\right] \sin \Delta=0 .
$$

Four types of stationary solutions exist namely the $\Delta=0, \Delta=\pi$, which we already observed in case of the low order energies, and the solutions

$$
\Delta= \pm \arccos \left(\frac{4\left(4 \mathcal{B}^{3}-7 \mathcal{B}^{2} \delta+\mathcal{B} \delta^{2}-\delta^{3}\right)}{\mathcal{B}\left(13 \mathcal{B}^{2}-8 \mathcal{B} \delta+6 \delta^{2}\right)}\right)
$$

which depends on $\mathcal{B}$ and $\delta$ and thus on the bias $\gamma$. The course of Eq. (4.99) as a function of $\gamma$ is shown in Fig. 4.10(b). Linear stability analysis shows that for $\tau \geq 0$ the $\Delta=0$ solution is unstable. The stability region of the $\Delta=\pi$ solution and the solution Eq. (4.99) is bias dependent. The bias dependent solution Eq. (4.99) is stable for $\gamma>\gamma^{*}$ and $\gamma<\gamma_{c}$ for which $\Delta=\pi$, see Fig. 4.10(b). For larger bias $\gamma>\gamma_{c}$ only the $d=\pi$ uniform solution is stable. 


\section{Bifurcation diagram}

The course of OP solutions when interacting with OD stripes is shown in Fig. 4.9(a,b). Similar to the low order variant of this coupling energy the amplitude of the stripes pattern $A_{1}$ is suppressed while the amplitude of the opposite mode $A_{1-}$ grows. Finally both amplitudes collapse, leading to a orientation scotoma solution. In contrast to the low order variant this stripe pattern is stable for arbitrary large inter-map coupling. In case of OP rhombic solutions inter-map coupling transforms this solution by reducing the amplitudes $A_{2}=A_{2^{-}}$ while increasing the amplitudes $A_{3}=A_{3^{-}}$. Without OD bias this solution is then transformed into the orientation scotoma stripe pattern, similar to the low order variant of this energy. In contrast to the low order energy, for non-zero bias the amplitudes $A_{2}$ and $A_{3}$ stay small but non-zero. The course of OP solutions when interacting with OD hexagons is shown in Fig. 4.9(c,d). For a small OD bias $\left(\gamma=\gamma^{*}\right)$ OP rhombic solutions decay into OP stripe-like patterns. These stripe-like patterns stay stable also for large-inter map coupling. In case of a larger OD bias $\left(\gamma=3 \gamma^{*}\right)$, both the OP stripe and the OP rhombic solutions decay into the uniform solution. Thus for small bias there is a bistability between stripe-like and uniform OP solutions while for larger OD bias the uniform solution is the only stable solution. The potential of OP stripe and OP rhombic solutions is shown in Fig. 4.9 (e,f). In the uncoupled case as well as for small inter-map coupling strength OP stripe solutions are for all bias values the energetic ground state. For large inter-map coupling and a small bias $\left(\gamma \approx \gamma^{*}\right)$ rhombic solutions are unstable and the stripe-like solutions are energetically preferred compared to PWC solutions. For larger bias, however, PWC solutions are the only stable solutions for large inter-map coupling. 

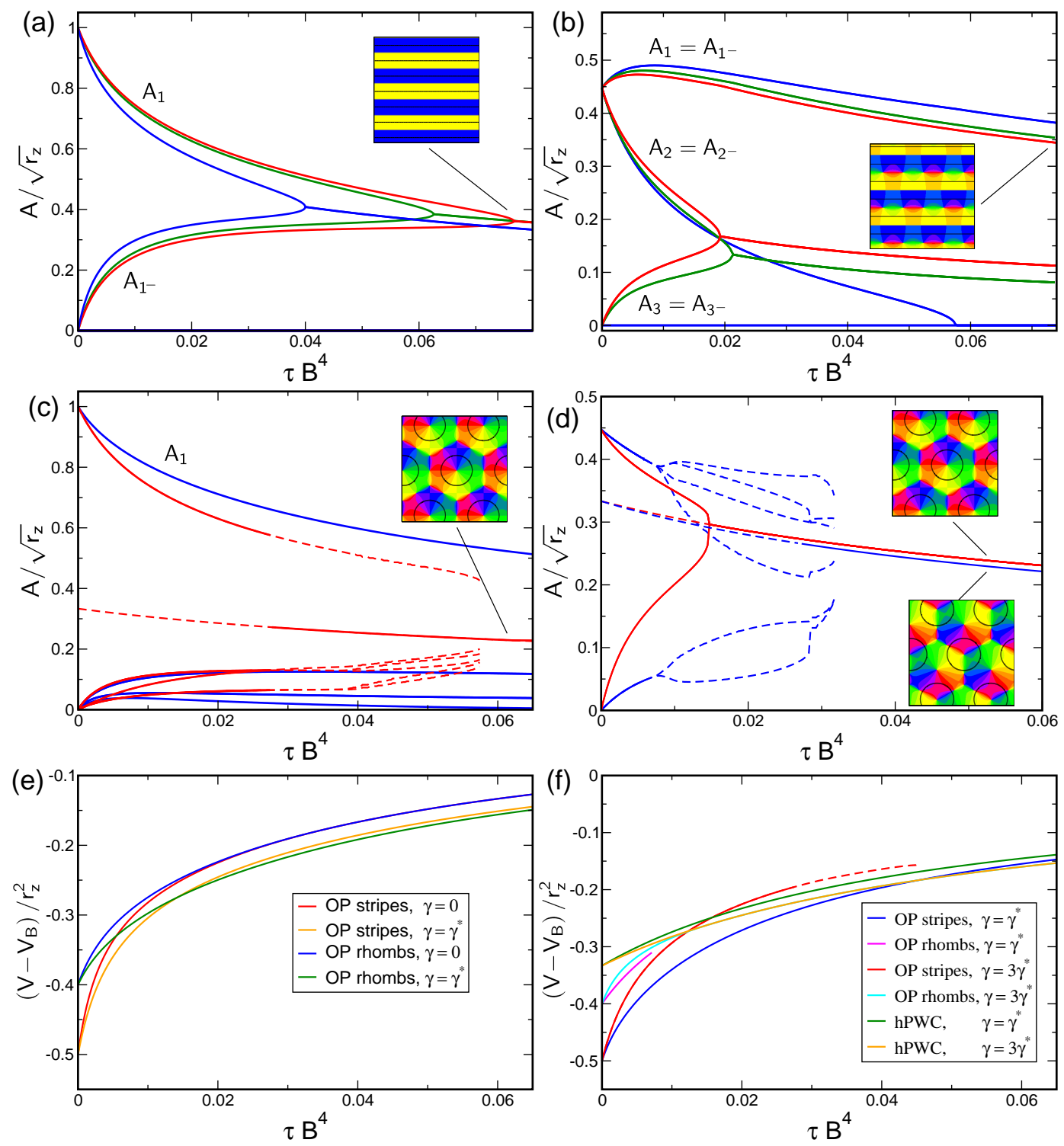

Figure 4.9.: Stationary amplitudes with coupling energy $\mathbf{U}=\tau|\mathbf{z}|^{4} \mathbf{o}^{4}$. Solid (dashed) lines: stable (unstable) solutions to Eq. (4.92). (a,b) OD stripes, $\gamma=0$ (blue), $\gamma=\gamma^{*}$ (green), $\gamma=1.4 \gamma^{*}$ (red). (c,d) OD hexagons, $\gamma=\gamma^{*}$ (blue), $\gamma=3 \gamma^{*}$ (red). (e) Potential, Eq. (4.91), of OP stripes and OP rhombs interacting with OD stripes. (f) Potential, Eq. (4.91), of OP stripes, OP rhombs, and hPWC interacting with OD hexagons. 

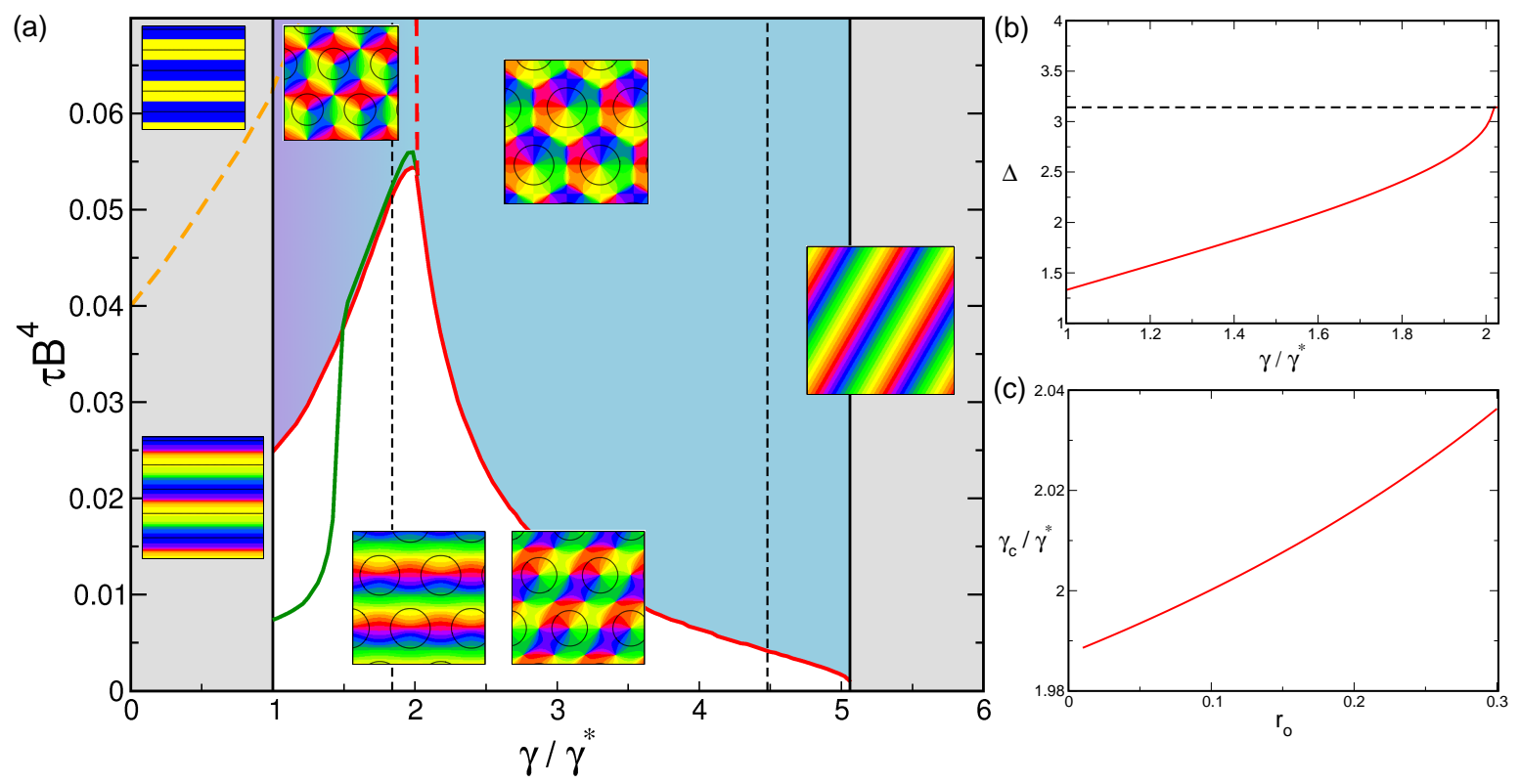

Figure 4.10.: (a) Phase diagram with coupling energy $\mathbf{U}=\tau \mathbf{o}^{\mathbf{4}}|\mathbf{z}|^{\mathbf{4}}, r_{o}=0.2, r_{z} \ll r_{o}$. Vertical black lines: stability range of OD stripes, hexagons, and constant solutions. Orange dashed line: Stability border of orientation scotoma stripes. Green solid line: Stability border of rhombic solutions. Red solid line: Stability border of PWC solutions, red dashed line: $\gamma_{c}$, (b) Course of Eq. (4.99), dashed line: $\Delta=\pi$. (c) Stability border between Eq. (4.99) solution and the $\Delta=\pi$ solution as a function of $r_{o}$ (vertical red line in (a)).

\section{Phase diagram}

The stability properties of all stationary solutions are summarized in the phase diagram Fig. 4.10. Compared to the gradient-type interaction energy we cannot scale out the dependence on $r_{o}$. The phase diagram is thus plotted for $r_{o}=0.2$. We rescale the inter-map coupling strength as $\tau \mathcal{B}^{4}$ where $\mathcal{B}$ is the stationary amplitude of the OD hexagons. In the regime of stable OD stripes there is a transition from OP stripes towards the orientation scotoma stripe solution. In the regime of stable OD hexagons there is a transition from OP stripes towards PWC solutions (red line). The stability border of PWC solutions is strongly OD bias dependent and has a peak at $\gamma \approx 2 \gamma^{*}$. For small OD bias $\gamma$ the uniform solution Eq. (4.99) is stable. With increasing bias there is a smooth transition of this solution until at $\gamma=\gamma_{c}$ the $d=\pi$ uniform solution becomes stable. In Fig. 4.10(c) the stability border $\gamma_{c}$ between the two types of uniform solutions is plotted as a function of $r_{o}$. We observe that there is only a small dependence on the control parameter and $\gamma_{c} \approx 2 \gamma^{*}$.

\section{Pinwheel crystals}

Figure 4.11 illustrates the uniform solution Eq. (4.99) for different values of the OD bias $\gamma$. For small bias, the OP pattern has six pinwheels per unit cell. Two of them are located at OD maxima while one is located at an OD minimum. The remaining three pinweels are located near the border of OD. With increasing bias, these three pinwheels are pushed further away from the OD border, being attracted to the OD maxima. With further increasing bias 

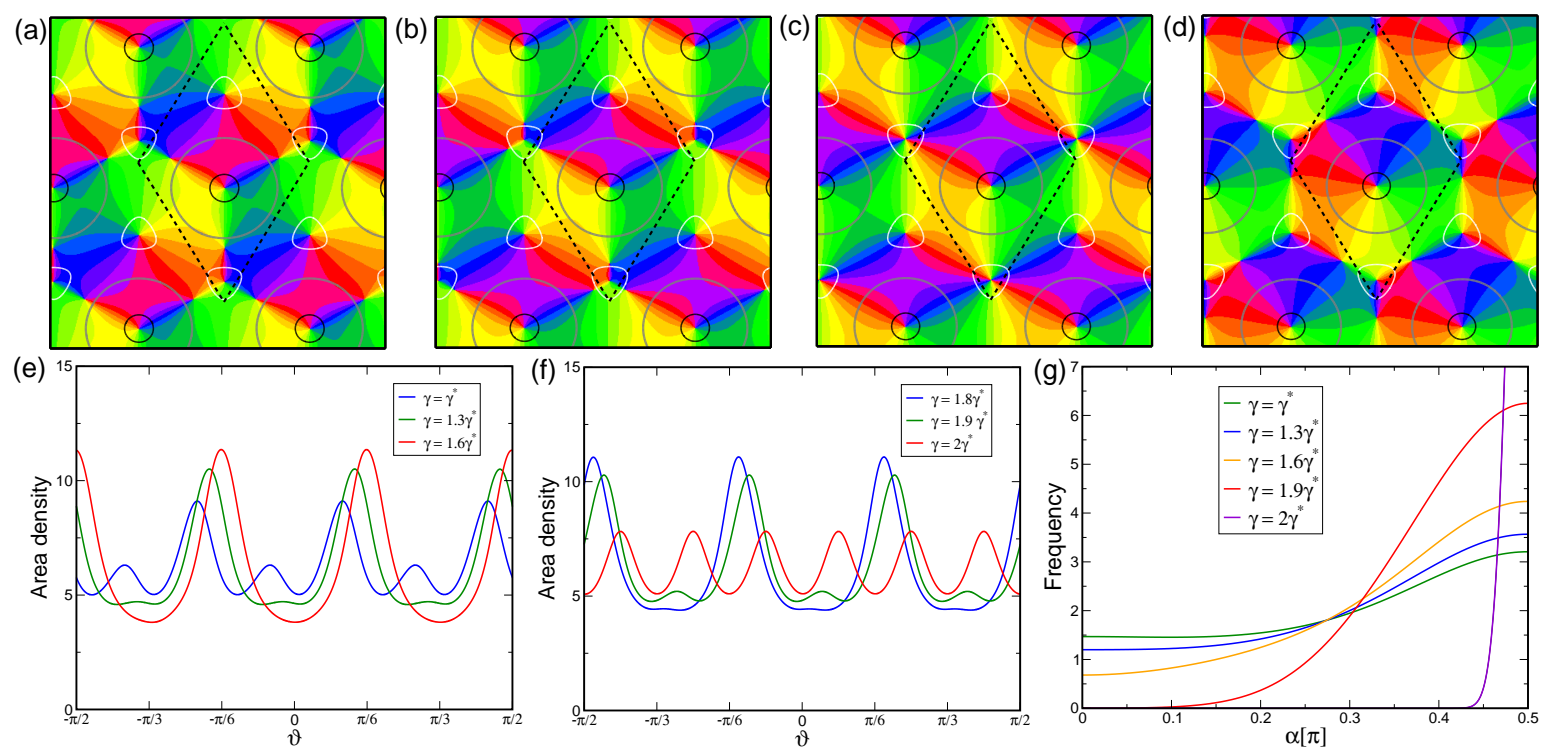

Figure 4.11.: Bias dependent pinwheel crystals, Eq. (4.99) (a) $\gamma=\gamma^{*}$, (b) $\gamma=1.3 \gamma^{*}$, (c) $\gamma=1.6 \gamma^{*}$, (d) $\gamma=2 \gamma^{*}$. OP map, superimposed are the OD borders (gray), $90 \%$ ipsilateral eye dominance (black), and $90 \%$ contralateral eye dominance (white), $r_{o}=0.2$. Dashed lines mark the unit cell of the regular pattern. (e,f) Distribution of orientation preference. (g) Intersection angles between iso-orientation lines and OD borders.

three wandering pinwheels merge with the one at the OD maximum building a single charge 1 pinwheel centered on a contra peak. The remaining two pinwheels are located at an ispi and contra peak, respectively. Note, compared to the Braitenberg PWC of the $\Delta=0$ uniform solution the charge 1 pinwheel here is located at the contralateral OD peak. Finally, the charge 1 pinwheels split up again into four pinwheels. With increasing bias the solution more and more resembles the Ipsi-center $\mathrm{PWC}(\Delta=\pi$ solution) which is stable also in the lower order version of the coupling energy. Finally, at $\gamma / \gamma^{*} \approx 2$ the Ipsi-center PWC becomes stable and fixed for $\gamma>2 \gamma^{*}$. The distribution of preferred orientations for different values of the bias $\gamma$ is shown in Fig. 4.11(e,f), reflecting the symmetry of each pattern. The distribution of intersection angles is shown in Fig. 4.11(g). The calculation of intersection angles is detailed in Section 5.8. Remarkably, all solutions show a tendency towards perpendicular intersection angles. This tendency is more pronounced with increasing OD bias. At about $\gamma / \gamma^{*} \approx 1.9$ parallel intersection angles are completely absent and at $\gamma / \gamma^{*} \approx 2$ there are exclusively perpendicular intersection angles.

\subsubsection{Gradient-type energy $U=\epsilon|\nabla o \nabla z|^{4}$}

Finally, we study the higher order version of the gradient-type inter-map coupling. The interaction terms are independent of the OD shift $\delta$. In this case the coupling strength can be rescaled as $\beta \mathcal{B}^{4}$ and is therefore independent of the bias $\gamma$. The bias in this case only determines the stability of OD stripes, hexagons or the constant solution. 


\section{Stationary solutions and their stability}

As for its lower order pendant a coupling to OD stripes is relatively easy to analyze. The energetically preferred solutions are OP stripes with the direction perpendicular to the OD stripes for which $U=0$. In case the OD map is a constant Eq. (4.31) the gradient-type inter-map coupling leaves the OP unaffected. The stationary states are therefore OP stripes running in an arbitrary direction. In case of OD hexagons we study uniform modes $A_{j}=$ $A_{j^{-}}=\mathcal{A}, B_{j}=\mathcal{B}$ and $\psi_{1}=\psi_{3}=0, \psi_{2}=\pi$. The imaginary part of Eq. (4.92) leads to equations for the phases $\phi_{j}$. We use the ansatz Eq. (4.81) which leads to the stationarity condition

$$
-5 \sin \Delta+13 \cos \Delta \sin \Delta=0 .
$$

The solutions are $\Delta=0, \Delta=\pi$, and $\Delta= \pm \arccos \left(\frac{5}{13}\right) \approx \pm 1.176$ where the stationary amplitude are given by

$$
\mathcal{A}=\sqrt{r_{z} /\left(9+\epsilon \mathcal{B}^{4}(61.875-7.5 \cos \Delta+4.875 \cos (2 \Delta))\right)} .
$$

In addition to these hexagonal pinwheel crystals there exist also non-uniform solutions. Besides stripe-like solutions of $z(\mathbf{x})$ with one dominant mode we find rhombic pinwheel crystals $\mathcal{A}_{j}=\mathcal{A}_{j^{-}}=(\mathcal{A}, a, \mathcal{A})$ with $a \ll \mathcal{A}$ and distorted rhombic crystals $\mathcal{A}_{j}=\left(\mathcal{A}_{1}, \mathcal{A}_{2}, \mathcal{A}_{3}\right), A_{j^{-}}=$ $\left(\mathcal{A}_{3}, \mathcal{A}_{2}, \mathcal{A}_{1}\right)$.

We calculate the stability properties of the uniform solution by linear stability analysis considering perturbations of the amplitudes $\mathcal{A}_{j} \rightarrow \mathcal{A}+a_{j}, \mathcal{A}_{j^{-}} \rightarrow \mathcal{A}+a_{j^{-}}$and of the phases $\phi_{j} \rightarrow \phi_{j}+\varphi_{j}, \phi_{j^{-}} \rightarrow \phi_{j^{-}}+\varphi_{j^{-}}$. This leads to a perturbation matrix $M$. In general amplitude and phase perturbations do not decouple. We therefore calculate the eigenvalues of the perturbation $M$ matrix numerically. It turns out that for this type of coupling energy only the solution with $\Delta= \pm \arccos \left(\frac{5}{13}\right)$ is stable.

\section{Bifurcation diagram}

For increasing inter-map coupling strength the amplitudes of the OP stripe and OP rhombic solutions are shown in Fig. 4.12(a). In case of stable OD hexagons there is a transition from rhombic pinwheel crystals (blue) towards distorted rhombic pinwheel crystals (green). The distorted rhombic PWC then decay into the hexagonal PWC (red). In case of OP stripes (black dashed lines) inter-map coupling leads to a slight suppression of the dominant mode and a growth of the remaining modes. This growth saturates at small amplitudes and thus the solution stays stripe-like. This stripe-like solution stays stable for arbitrary large inter-map coupling. Therefore there is a bistability between hexagonal PWC solutions and stripe-like solutions for large inter-map coupling.

The stability borders for the rhombic PWC and distorted rhombic PWC solutions were obtained by calculating their bifurcation diagram numerically from the amplitude equations Eq. (4.92). With increasing map coupling we observe a transition from a rhombic PWC (two large amplitudes) to a distorted rhombic PWC (three different amplitudes) at $\epsilon \mathcal{B}^{4} \approx 0.033$ 

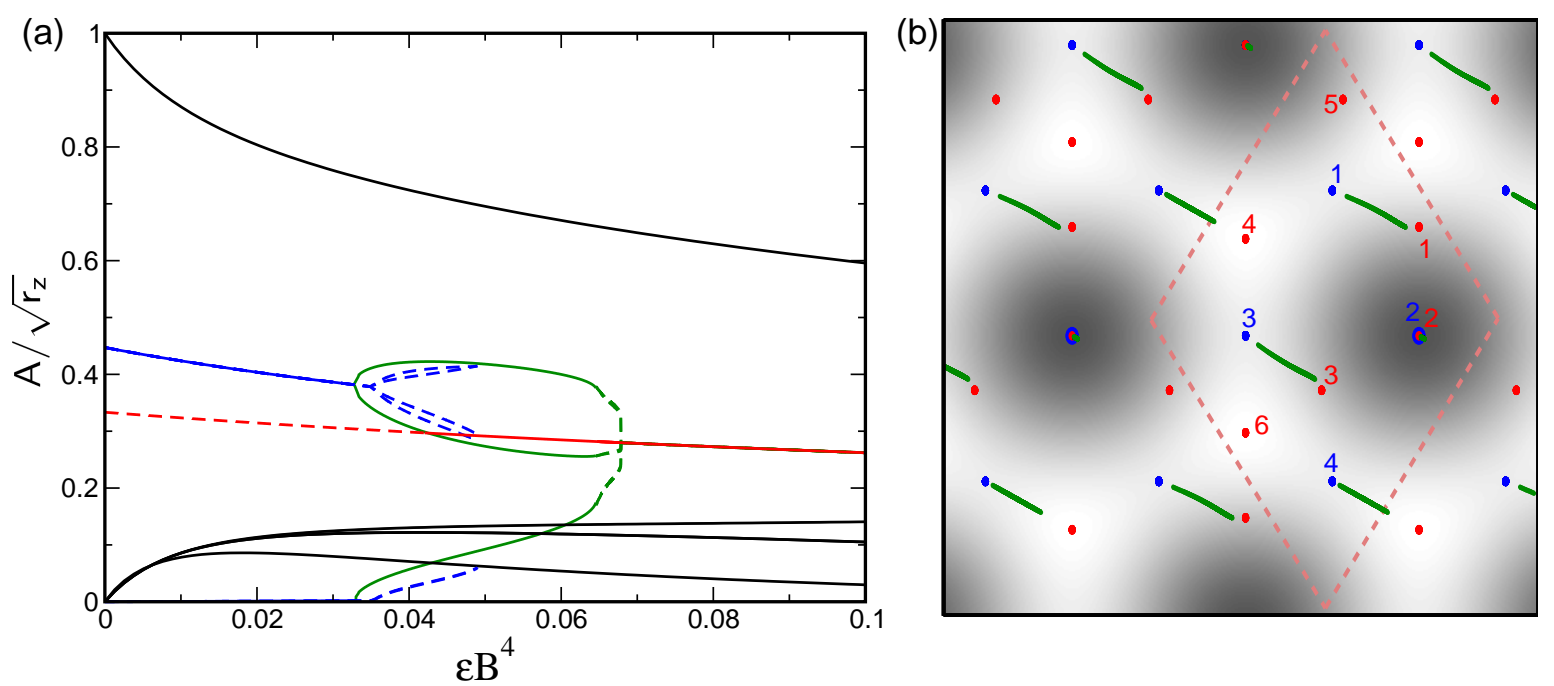

Figure 4.12.: Stationary amplitudes with coupling energy $\mathbf{U}=\epsilon|\nabla \mathbf{z} \nabla \mathbf{o}|^{4}$, (a) Solid (dashed) lines: Stable (unstable) solutions of Eq. (4.92). Blue: rhombic PWC, green: distorted rhombic PWC, red: hexagonal PWC. Black lines: stripe-like solutions. (b) OD map, superimposed pinwheel positions for different coupling strengths, $\gamma / \gamma^{*}=3$. Dashed lines: unit cell.

(blue dashed line in Fig. 4.13(a)), see also Fig. 4.12(a). The distorted rhombic PWC loses its stability at $\epsilon \mathcal{B}^{4} \approx 0.065$ (blue solid line in Fig. 4.13(a)) and from thereon all amplitudes are equal corresponding to the hexagonal PWC. There is a bistability between hexagonal PWC, rhombic PWC, and stripe-like solutions. To calculate the inter-map coupling needed for the hexagonal solution to become the energetic ground state we calculated the potential Eq. (4.91) for the three solutions. In case of the uniform solution Eq. (4.81) the potential is given by

$$
\begin{aligned}
V= & -6 \mathcal{A}^{2} r_{z}-3 \mathcal{B}^{2} r_{o}+27 \mathcal{A}^{4}+\frac{45}{2} \mathcal{B}^{4} \\
& +\frac{1}{16} \mathcal{A}^{4} \mathcal{B}^{4} \epsilon(3210-456 \cos \Delta+90 \cos (2 \Delta))
\end{aligned}
$$

The potential in case of the rhombic and stripe-like solutions is obtained by numerically solving the amplitude equations when using initial conditions close to these solutions. Above $\epsilon \mathcal{B}^{4} \approx 0.12$ the hexagonal PWC is energetically preferred compared to stripe-like solutions (red dashed line in in Fig. 4.13(a)) and thus corresponds to the energetic ground state for large inter-map coupling.

\section{Phase diagram}

We calculate the phase diagram of the coupled system in the limit $r_{z} \ll r_{o}$, shown in Fig.4.13, The phase diagram contains the stability borders of the uncoupled OD solutions $\gamma^{*}, \gamma_{2}^{*}, \gamma_{3}^{*}, \gamma_{4}^{*}$. They correspond to vertical lines, as they are independent of the inter-map coupling in the limit $r_{z} \ll r_{o}$. At $\gamma=\gamma^{*}$ hexagons become stable. Stripe solutions become unstable at $\gamma=\gamma_{2}^{*}$. At $\gamma=\gamma_{3}^{*}$ the homogeneous solution becomes stable while at $\gamma=\gamma_{4}^{*}$ hexagons loose 


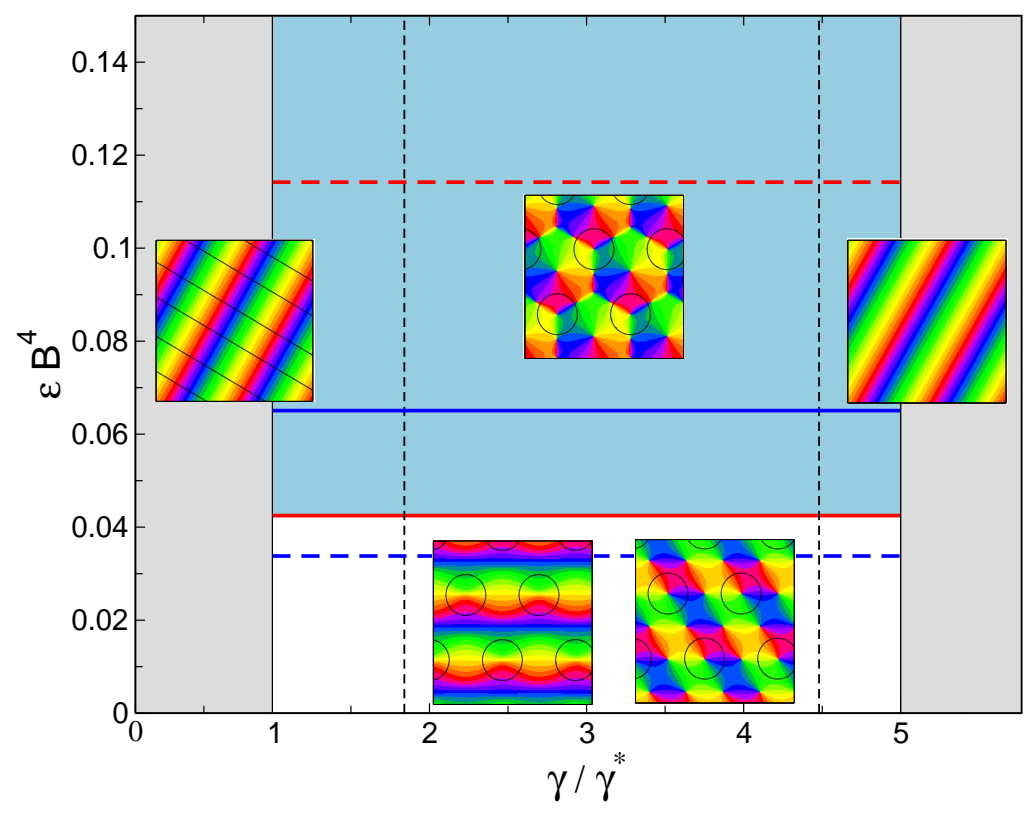

Figure 4.13.: Phase diagram with coupling energy $\mathbf{U}=\epsilon|\nabla \mathbf{z} \nabla \mathbf{o}|^{4}$, for $r_{z} \ll r_{o}$. Vertical lines: stability range of OD hexagons, red solid line: stability border of hexagonal PWC, blue solid line: stability border of rhombic PWC, blue dashed line: transition from rhombic to distorted rhombic PWC. Dashed red line: hexagonal PWC corresponds to ground state of energy.

their stability. In the units $\gamma / \gamma^{*}$ the borders $\gamma_{2}^{*}, \gamma_{3}^{*}, \gamma_{4}^{*}$ vary slightly with $r_{o}$, see Fig. 4.2, and are drawn here for $r_{o}=0.2$. We rescale the inter-map coupling strength as $\epsilon \mathcal{B}^{4}$ where $\mathcal{B}$ is the stationary amplitude of the OD hexagons. The stability borders of OP solutions are therefore horizontal lines. For $\gamma<\gamma^{*}$ or for $\gamma>\gamma_{4}^{*}$ pinwheel free orientation stripes are dynamically selected. For $\gamma^{*}<\gamma<\gamma_{4}^{*}$ and above a critical effective coupling strength $\epsilon \mathcal{B}^{4} \approx 0.042$ hexagonal PWC solutions are stable and become the energetic ground state of Eq. (4.91) above $\epsilon \mathcal{B}^{4} \approx 0.117$. Below $\epsilon \mathcal{B}^{4} \approx 0.065$, rhombic PWC solutions are stable leading to a bistability region between rhombic and hexagonal PWC solutions. We find in this region that rhombic solutions transform into distorted rhombic solutions above an effective coupling strength of $\epsilon \mathcal{B}^{4} \approx 0.033$.

\section{Pinwheel crystals}

First, we study the spatial layout of the rhombic solutions which is illustrated in Fig. 4.14, The rhombic PWC solutions are symmetric under rotation by 180 degree. The rhombic solution has 4 pinwheels per unit cell and its pinwheel density is thus $\rho=4 \cos (\pi / 6) \approx 3.5$. One may expect that the energy term Eq. (3.23) favors pinwheels to co-localize with OD extrema. In case of the rhombic layout there is only one pinwheel at an OD extremum while the other three pinwheels are located at OD saddle-points which are also energetically favorable positions with respect to $U$. The orientation selectivity $|z(\mathbf{x})|$ for the rhombic PWC is shown in Fig. 4.14(b). The pattern of selectivity is arranged in small patches of highly 
(a)
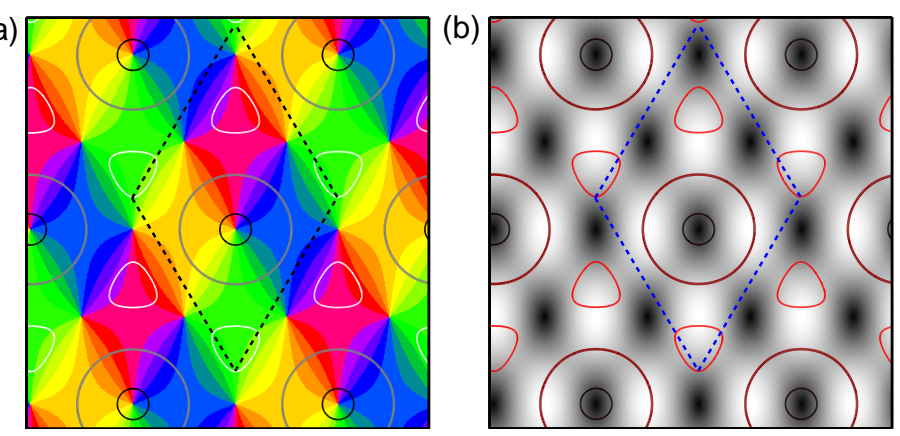

Figure 4.14.: Rhombic pinwheel crystals. (a) OP map with superimposed OD borders (gray), 90\% ipsilateral eye dominance (black), and 90\% contralateral eye dominance (white), $\gamma=3 \gamma^{*}, r_{o}=0.2$. (b) Selectivity $|z(\mathbf{x})|$, white: high selectivity, black: low selectivity.

selective regions. Next, we study the hexagonal layout of the two stable uniform solutions which is shown in Fig. 4.15. The $\Delta= \pm \arccos (5 / 13)$ solutions have six pinwheels per unit cell. Their pinwheel density is therefore $\rho=6 \cos \pi / 6 \approx 5.2$. Three pinwheels of the same topological charge are located at the extrema of the OD map. Two of these are located at the OD maximum while one is located at the OD minimum. The remaining three pinwheels are not at an OD extremum but near the OD border. The distance to the OD border depends on the OD bias, see Fig. $4.15(d)$. For a small bias $\left(\gamma \approx \gamma^{*}\right)$ these three pinwheels are close to the OD borders and with increasing bias the OD border moves away from the pinwheels. The pinwheel in the center of the OP hexagon is at the contralateral OD peak. Because these pinwheels organize most of the map while the others essentially only match one OP hexagon to its neighbors we refer to this pinwheel crystal as the Contra-center pinwheel crystal. Note, that some pinwheels are located at the vertices of the hexagonal pattern. Pinwheels located between these vertices (on the edge) are not in the middle of this edge. Solutions with $\Delta= \pm \arccos (5 / 13)$ are therefore not symmetric under a rotation by 60 degree but symmetric under a rotation by 120 degree. Therefore the solution $\Delta=+\arccos (5 / 13)$ cannot be transformed into the solution $\Delta=-\arccos (5 / 13)$ by a rotation of the OD and OP pattern by 180 degrees. This symmetry is also reflected by the distribution of preferred orientations, see Fig. 4.15(f). Six orientations are slightly overrepresented. Compared to the Ipsi-center PWC, which have a $60^{\circ}$ symmetry, this distribution illustrates the $120^{\circ}$ symmetry of the pattern. Next, we study the transition from a rhombic PWC towards the Contra-center PWC. We tracked the pinwheel positions for each value of the inter-map coupling strength, see Fig. 4.12(b). In the regime where the distorted rhombic PWC is stable, three of the four pinwheels of the rhombic PWC are moving either from an OD saddle-point to a position near an OD border (pinwheel 1 and 3) or from an OD saddle-point to an OD extremum (pinwheel 4). One pinwheel (pinwheel 2) is fixed in space. At the transition to hexagonal PWC the two additional pinwheels show up, one near an OD border (pinwheel 5) and one at an OD extremum (pinwheel 6).

The distribution of intersection angles is continuous, see Fig. 4.15(c). Although there is a fixed uniform solution with varying OD bias the distribution of intersection angles changes. The 
(a)

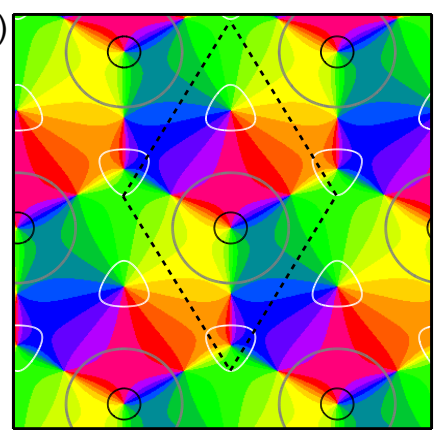

(d)

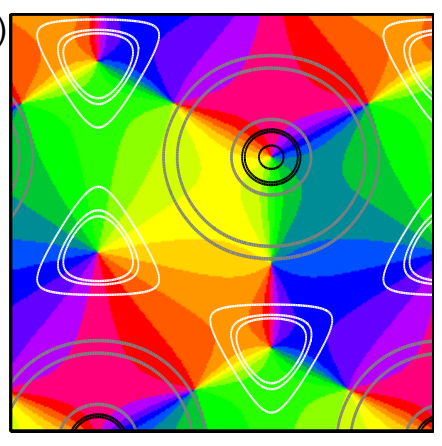

(b)

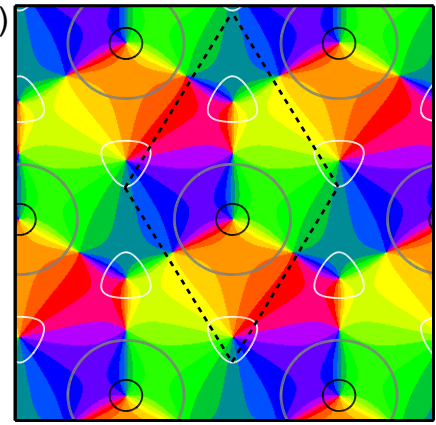

(e)

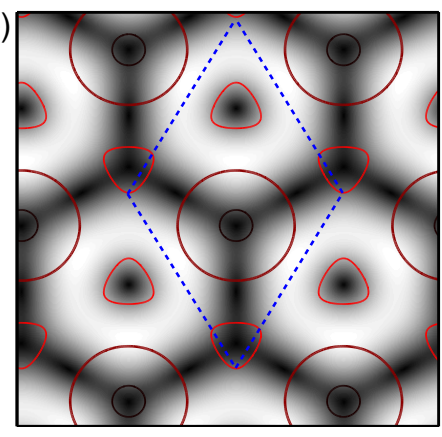

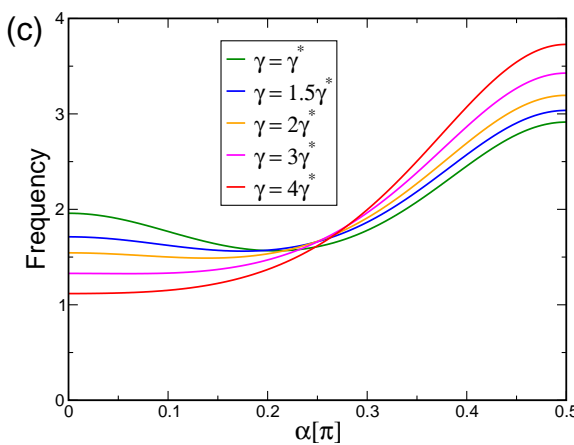

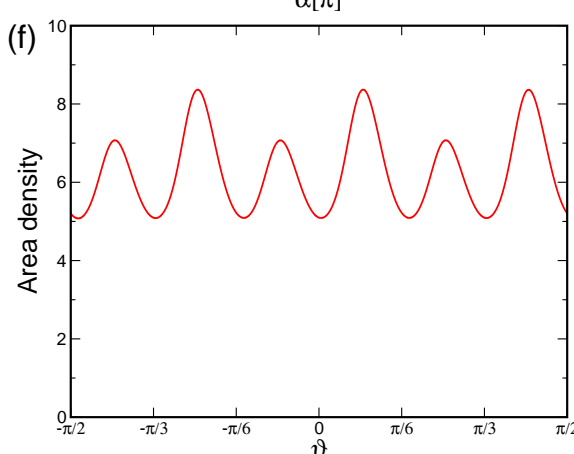

Figure 4.15.: Contra-center pinwheel crystals. (a,b) OP map, superimposed are the OD borders (gray), 90\% ipsilateral eye dominance (black), and $90 \%$ contralateral eye dominance (white), $r_{o}=0.2, \gamma=3 \gamma^{*}$. (a) $\Delta=\arccos (5 / 13)$, (b) $\Delta=-\arccos (5 / 13)$. (c) Distribution of orientation preference. (d) OP map with superimposed OD map for three different values $\left(\gamma=\gamma^{*}, \gamma=\left(\gamma_{4}^{*}-\gamma^{*}\right) / 2+\gamma^{*}, \gamma=\gamma_{4}^{*}\right)$ of the OD bias. (e) Selectivity $|z(\mathbf{x})|$, white: high selectivity, black: low selectivity. (f) Distribution of intersection angles.

reason for this is the bias dependent change in the OD borders, see Fig. 4.15(d). For all bias values there is a tendency towards perpendicular intersection angles, although for low OD bias there is an additional small peak at parallel intersection angles. The orientation selectivity $|z(\mathbf{x})|$ for the hexagonal PWC is shown in Fig. 4.15)(e). The pattern shows hexagonal bands of high selectivity.

To summarize, we discussed the impact of inter-map coupling on the layout of OP map using four representative inter-map coupling energies. The results show that for substantial bias towards one eye pinwheels are in fact stabilized and pinwheel rich solutions become energetic ground states by inter-map coupling.

\subsection{Numerical analysis of pinwheel crystallization}

So far, we presented an analytical treatment of the map interaction model. We showed that for higher order inter-map coupling energies we can neglect the backreaction onto the OD map for sufficiently small ratio $r_{z} / r_{o}$. To test whether the presented solutions and their stability ranges revealed for $r_{z} \ll r_{o}$ persist when the backreaction on the OD map is taken into account we solve the full field dynamics Eq. (4.1) numerically. For details of the numerical methods see Section 3.3 and Appendix A.1. Besides the influence of the backreaction the 
(a)
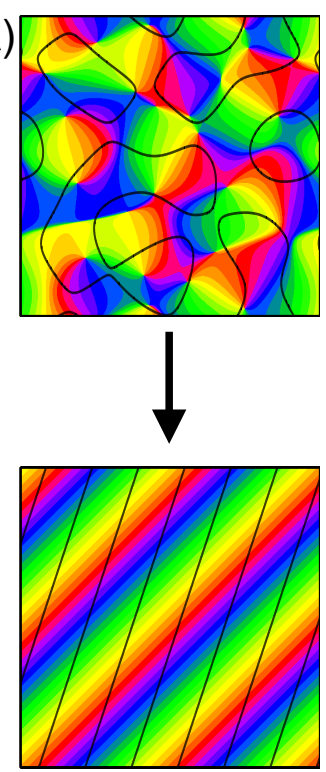

(b)
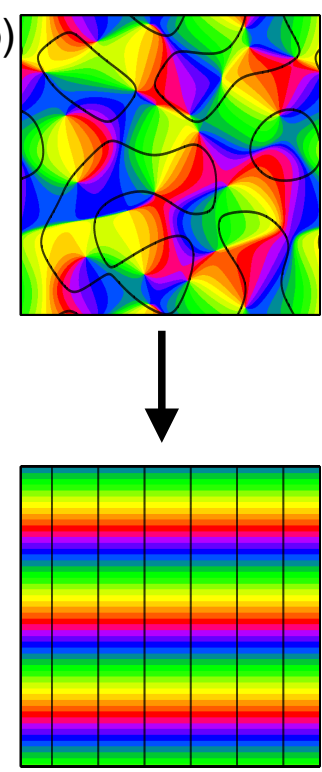

(c)
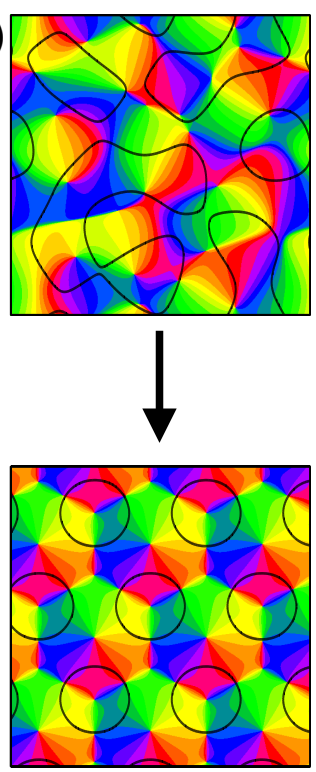

(d)
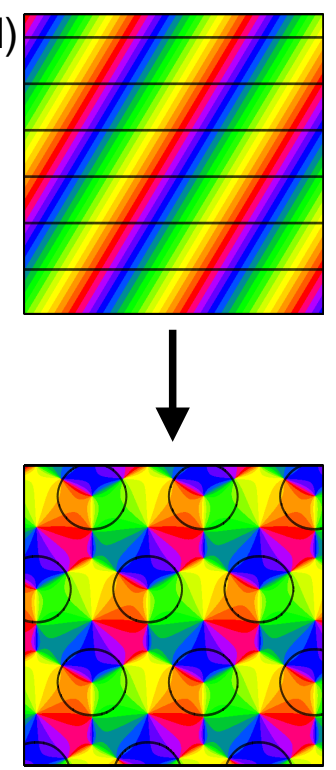

Figure 4.16.: Pinwheel annihilation, preservation, and generation in numerical simulations for different strengths of inter-map coupling and OD bias, $r_{o}=0.2, r_{z}=0.02$. OP map with zero contours of OD map superimposed. (a) $\gamma=0, \epsilon=0$ (b) $\gamma=0, \epsilon=2000$ (c) and (d) $\gamma=0.15, \epsilon=2000$. In all conditions: $T_{f}=10^{4}, \Gamma=22,128 \times 128$ mesh. Initial conditions identical in (a) - (c).

full dynamical system receives additional corrections. There are higher order corrections to the uncoupled amplitude equations which become important for a substantially large control parameter $r_{z}, r_{o}$ but which we neglected here. When solving the system numerically we expect further corrections due to the spatial discretization of the dynamics.

Numerical simulations of the dynamics Eq. (4.1) with the higher order gradient-type coupling energy Eq. (3.23) are shown in Fig. 4.16. The initial condition and the final states are shown for different bias terms $\gamma$ and inter-map couplings $\epsilon$. We observe that for substantial contralateral bias and above a critical coupling $\epsilon$ pinwheels are preserved from random initial conditions or are generated if the initial condition is pinwheel free. Without a contralateral bias the final states are pinwheel free stripe solutions irrespective of the strength of the intermap coupling.

\subsubsection{Pinwheel kinetics}

To study the process of pinwheel annihilation, preservation, and creation we calculate the pinwheel density as well as various other pinwheel statistics, see Section 3.4. during time evolution. Simulations for the time evolution of the pinwheel density are shown in Fig. 4.17. In the uncoupled case $(\epsilon=0)$ most of the patterns decay into a stripe solution and their pinwheel density drops to a value near zero. At small coupling strengths $(\epsilon=200)$ the pinwheel density converges either to zero (stripes), to values near 3.5 for the rhombic PWC, or to approximately 5.2 for the contra-center PWC. At high map coupling $(\epsilon=2000)$ pinwheel free stripe patterns form neither from pinwheel rich nor from pinwheel free initial conditions. 
(a) $\rho$

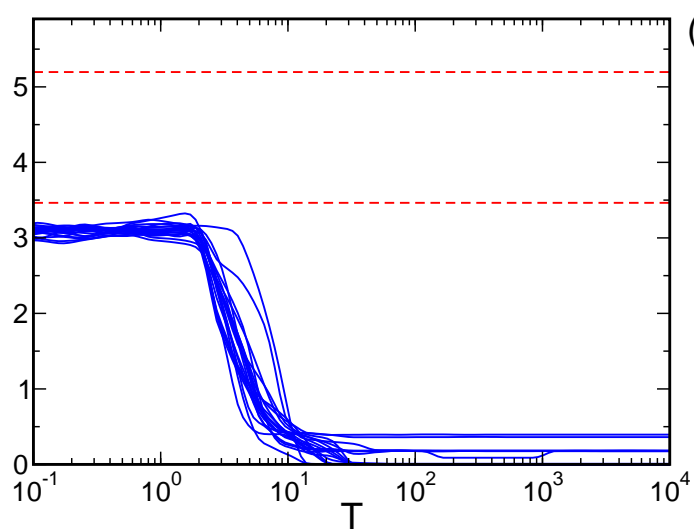

(b) $\rho$

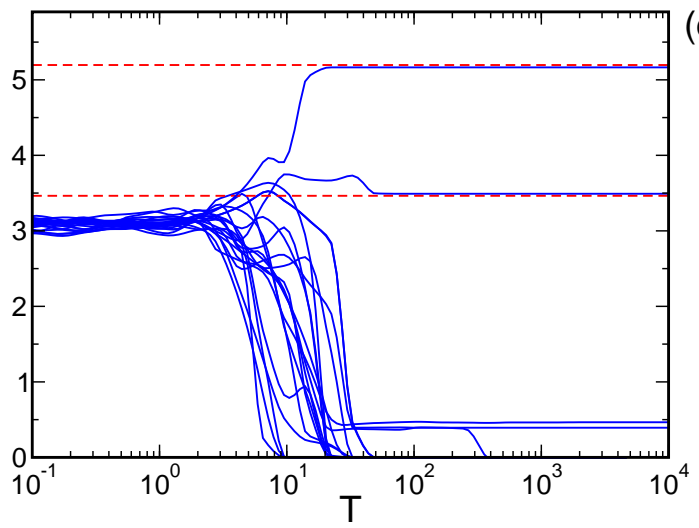

(c) $\rho$

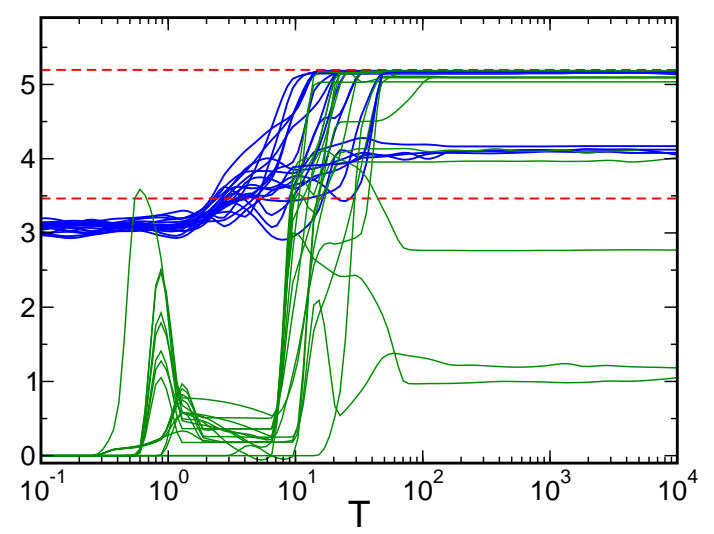

(d)

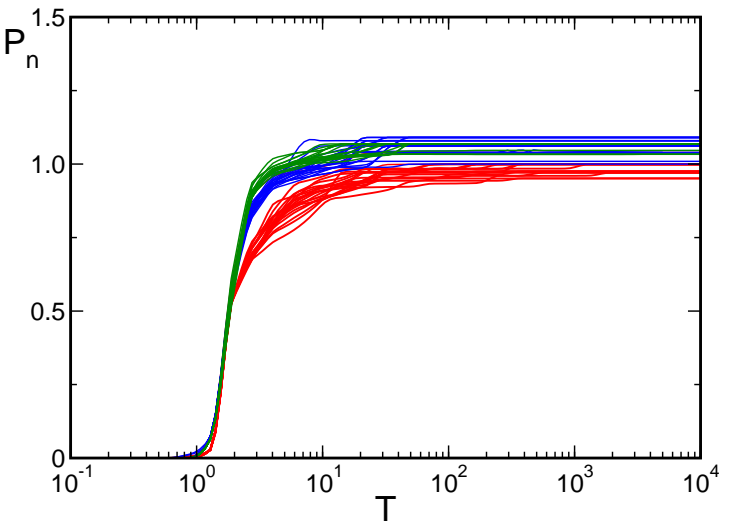

Figure 4.17.: Time evolution of the pinwheel density, $r_{z}=0.05, r_{o}=0.25, \gamma=0.15$. For each parameter set (a)-(c) simulations in blue started from an identical set of 20 initial conditions. Red dashed line: $\rho=4 \cos (\pi / 6)$ and $\rho=6 \cos (\pi / 6)$. (a) $\epsilon=0$ (b) $\epsilon=200$ (c) $\epsilon=2000$. (d) Normalized power of OP map, $\epsilon=0$ (red), $\epsilon=200$ (blue), and $\epsilon=2000$ (green). In green (c): OD and OP stripes as initial conditions. Parameters: $128 \times 128$ mesh, $\Gamma=22$.

In this regime the dominant layout is the contra-center PWC. When starting from OD and OP stripes, see Fig. 4.17(c) (green lines), the random orientation between the stripes first evolves towards a perpendicular orientation $(T \approx 1)$. This leads to a transient increase in the pinwheel density. At the time $(T \approx 10)$ where the OD stripes dissolve towards OD hexagons hexagonal PWC solutions form and the pinwheel density reaches its final value.

Regions of hexagonal PWC layout can however be inter-digitated with long lived rhombic PWC solutions and stripe domains. Figure 4.17(d) shows the time course of the normalized power $P_{n}(t)=\left\langle\left|z(\mathbf{x}, t)_{\mathrm{dyn}}\right|^{2}\right\rangle_{\mathbf{x}} /\left\langle\left|z(\mathbf{x}, t)_{\mathrm{th}}\right|^{2}\right\rangle_{\mathbf{x}}$, where \langle\rangle$_{\mathbf{x}}$ denotes spatial average. The field $z_{\mathrm{th}}$ is obtained from solution of the amplitude equations Eq. (4.67) while $z_{\text {dyn }}$ is the field obtained from the simulations. Starting from a small but nonzero power the amplitudes grow and saturate after $T \approx 1$. When the amplitudes are saturated the selection of the final pattern starts. Quantitatively, we find that with backreaction the critical coupling strengths are slightly increased compared to their values in the limit $r_{z} \ll r_{o}$. The inclusion of the backreaction is studied in detail in Chapter 5. Snapshots of the simulation leading to hexagonal PWC solutions at three time frames are shown in Fig. 4.18. At $T \approx 0.8$ a broad rearrangement of the pattern takes place and we can identify different domains in the pattern. 


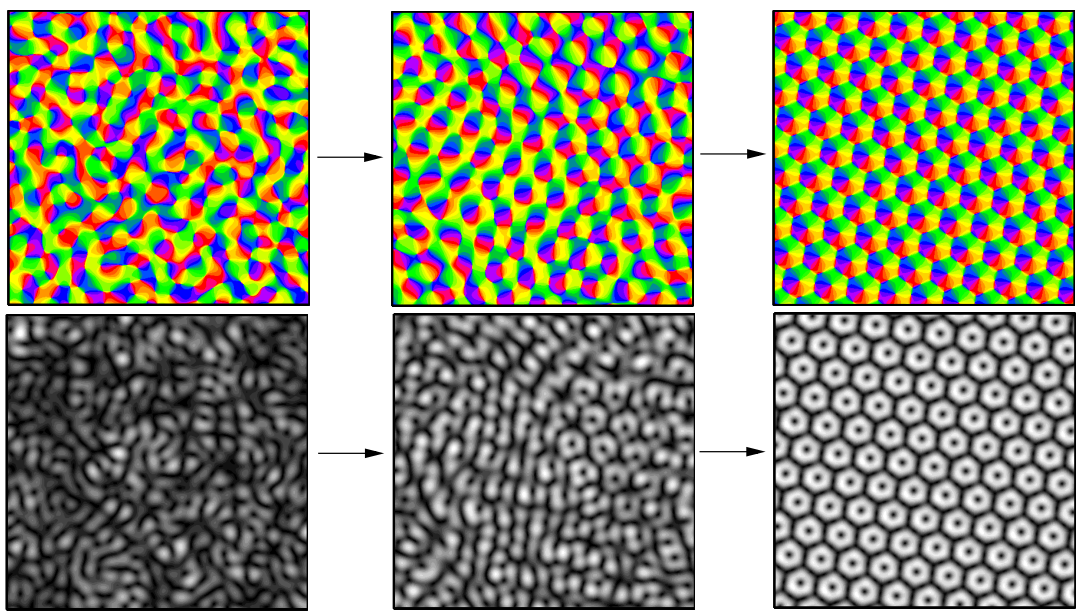

Figure 4.18.: Snapshots of the pinwheel crystallization process. Top panel: OP map, bottom panel: selectivity $|z(\mathbf{x})|$. Left: $T=0.01$, middle: $T=0.8$, right: $T=T_{f}=10^{4}$. Parameters as in Fig. 4.17(c).

During time evolution we further calculated the minimal distance between the pinwheels $d$, measured in units of the column spacing $\Lambda$. The distributions of distances for two simulations leading to rhombic and hexagonal PWC solutions are shown in Fig. 4.19, We can identify three stage in the evolution of the pinwheel distances. At early stages of the evolution $\left(T \approx 10^{-2}\right.$ ) there is a continuous distribution starting approximately linearly from $d=0$. At the time where the amplitudes saturate $(T \approx 1)$ the distribution of pinwheels becomes very inhomogeneous. Different domains with stripe-like, rhombic, or hexagonal patterns show up (see also Fig. $4.21(\mathrm{c}, \mathrm{d})$ ) until finally the rhombic or hexagonal pattern takes over the whole area.

As pinwheels carry a topological charge we can divide the distributions according to distances between pinwheels of the same charge or according to distances between pinwheels of the opposite charge. In Fig. 4.20 we have a focus on pinwheel distances for the final states of the dynamics. In case of the rhombic solutions there is only a single pinwheel to pinwheel distance with $d=1 / \sqrt{3} \approx 0.58 \Lambda$. In numerical simulations small variations in the amplitudes lead to a slightly larger distance between pinwheels of equal charge than between pinwheels of opposite charge. Therefore their distance distributions do not collapse exactly, see Fig. 4.20(a). In case of the hexagonal PWC there are three peaks at $d \approx 0.28 \Lambda, d \approx 0.36 \Lambda$ and $d \approx 0.56 \Lambda$ in the pinwheel distance distribution of arbitrary charge, see Fig. 4.20(b). These three peaks all result from distances between pinwheels carrying the opposite charge while the distance between pinwheels of the same charge shows two peaks at $d \approx 0.48 \Lambda$ and $d \approx 0.64 \Lambda$ in the distribution. The origin of the peaks is illustrated in Fig. 4.20(c) and Fig. 4.20(d). We have shown that inter-map coupling leads to a stabilization of pinwheels in the OP pattern. But to what extent are the pinwheels in the crystalline OP maps rearrangements of pinwheels of the initial OP pattern? To answer this question we calculated the pinwheel annihilation and creation rate during time evolution. The pinwheel creation $c(t)$ and annihilation $a(t)$ rates per hypercolumn are defined in Section 3.4. The time evolution of these rates, averaged 
(a)

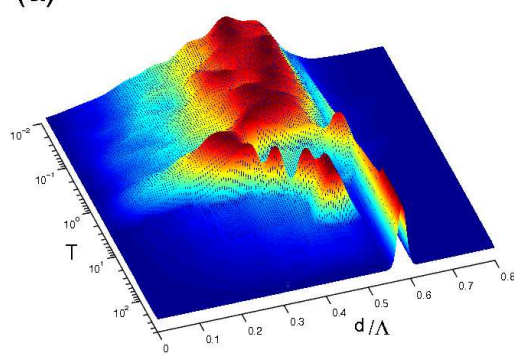

(d)

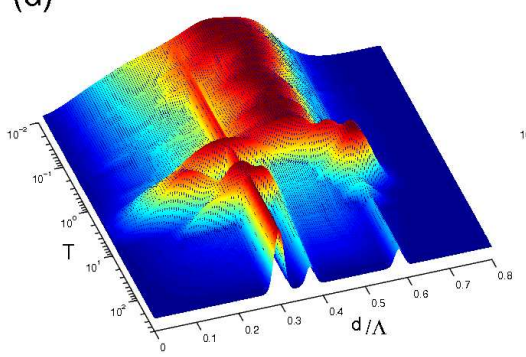

(b)

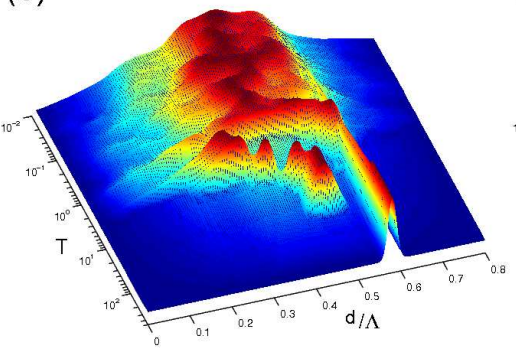

(e)

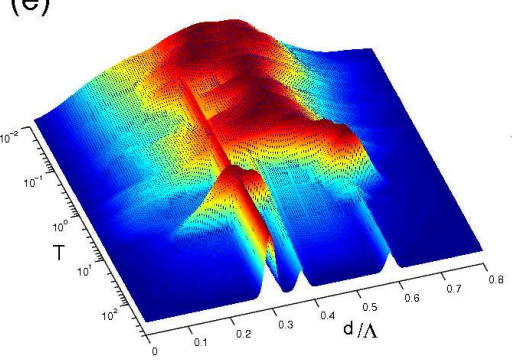

(c)

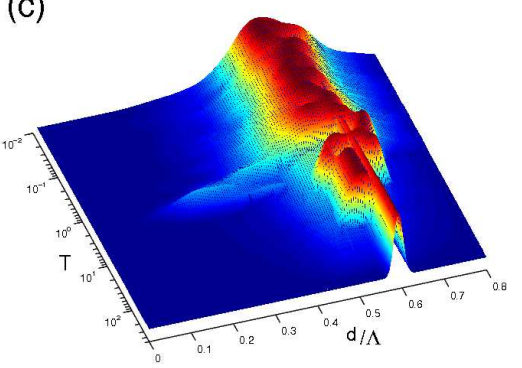

(f)

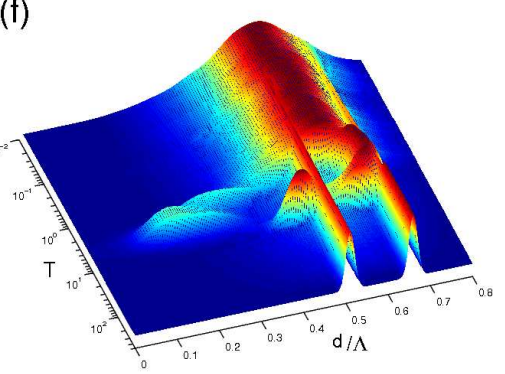

Figure 4.19.: Distribution of nearest neighbor distances during development. (a-c) rhombic PWC (d-f) hexagonal PWC. Distance to the next pinwheel of arbitrary (a,d), opposite (b,e), and equal (c,f) topological charge. Parameters as in Fig. 4.17(b).

over 20 simulations leading to a hexagonal PWC, is shown in Fig. 4.21(a). We observe that both rates are fairly equal throughout the development, with a slightly higher creation rate in the later stage of development. During the initial stages of time evolution creation and annihilation rates decay algebraically. At $T \approx 3$ both rates deviate from this algebraic decay. From thereon annihilation and creation rates increase, reflecting a broad rearrangement of the pattern. After $T \approx 15$ no pinwheels are created or annihilated anymore and the pinwheels of the final pattern are present.

Pinwheels are created and annihilated until the crystal pattern is formed. How many pinwheels of the initial pattern are thus present in the final pattern? For a given set of pinwheels at an initial time $T=T^{*}$ we further calculate the fraction $s(t)$ of those pinwheels surviving until time $T$. The fraction of pinwheels present at time $T^{*}$ that survive up to the final time $T=T_{f}$ is given by $p(t)$. Both fractions are shown in Fig. 4.21(b) for $T^{*}=0.01$ and in Fig. 4.21 (c) for $T^{*}=2$, a time where the power $P(t)$ has almost saturated, see Fig. 4.17(d). We observe that about $20 \%$ of the initial pinwheels are preserved until the final time and therefore most of the pinwheels of the crystal pattern are created during development. From those pinwheels which are present when the power saturates about $65 \%$ are also present in the final pattern.

\subsubsection{Detuning OD and OP wavelengths}

The previous analytical as well as numerical results show that OD stripes are not capable of stabilizing pinwheels. In case of gradient-type inter-map couplings the OP map consists of stripes which run perpendicular to the OD stripes, see Fig. 4.7 and Fig. 4.13)(a). In case 

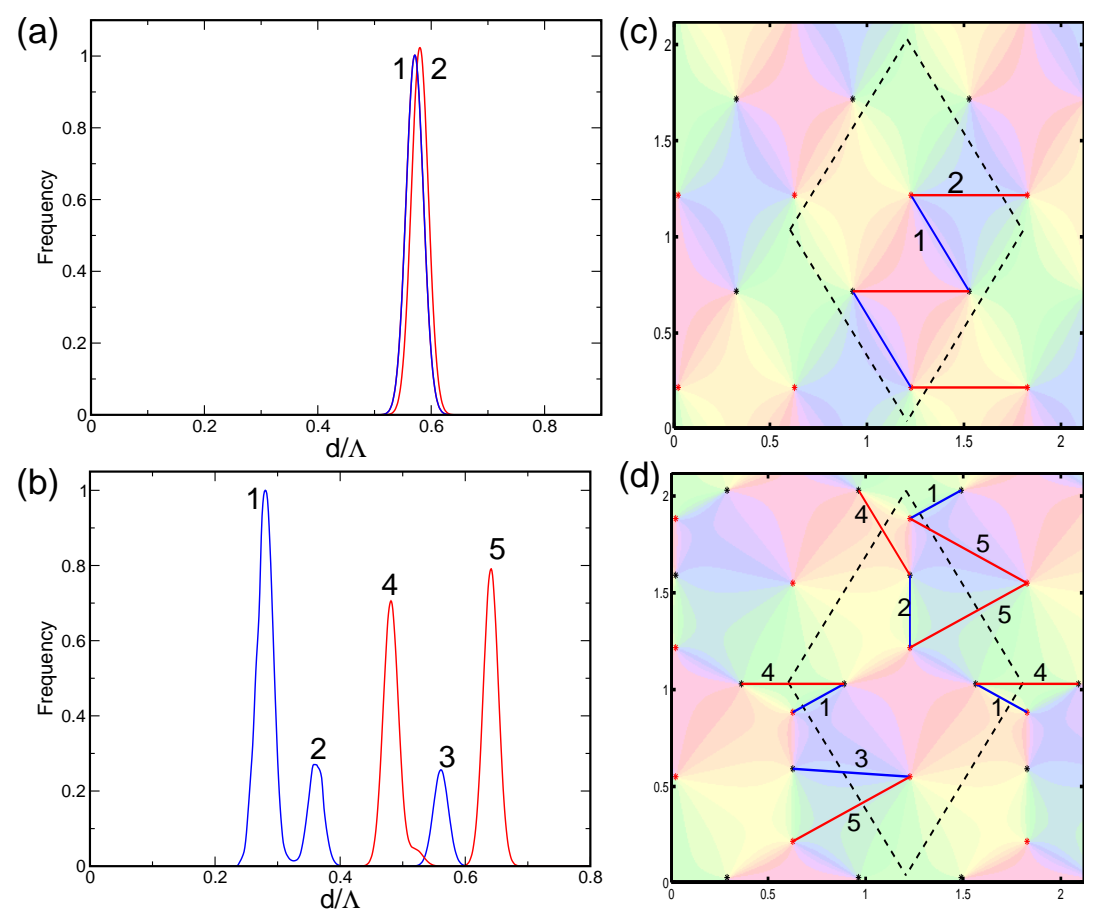

Figure 4.20.: Distribution of nearest neighbor distances for final states

$\left(T=T_{f}=10^{4}\right)$. (a) rhombic PWC, (b) hexagonal PWC with pinwheels of equal (red) and opposite (blue) charge. (c) and (d) Illustration of occurring pinwheel distances. Pinwheels are marked with star symbols according to their charge. Units are given in $\Lambda$. Parameters as in Fig. 4.17(b). 

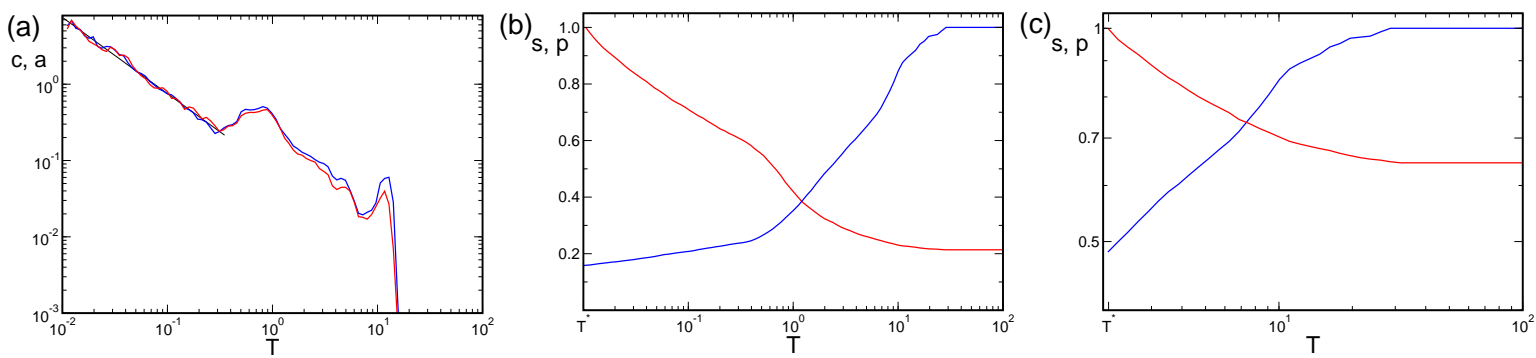

Figure 4.21.: Pinwheel annihilation and creation. (a) Creation (blue) and annihilation (red) rates during time evolution. (b,c) Survival fraction (red) and fraction of preserved pinwheels (blue) compared to the initial time $T^{*}=0.01$ (b) and $T^{*}=2$ (c). Parameters as in Fig. 4.17(c).

of the product-type inter-map coupling high gradient regions of both maps avoid each other by producing again OP stripes but now running in the same direction as the OD stripes, see Fig. 4.3(a) and Fig. 4.9(a). In numerical simulations we also investigate the case of OD stripes of larger wavelength than OP columns, as is the case in macaque monkeys [75]. In case of a gradient-type inter-map coupling the OD bands are perpendicular to the OP bands independent of the ratio $\Lambda_{o} / \Lambda_{z}>1$, see Fig. 4.22(a-c). In case of the product-type intermap coupling if the ratio $\Lambda_{o} / \Lambda_{z}>1$ orientation representation does not collapse, as seen in Fig. 4.9(d-f). The system, however, again finds a way to put a zero contour of $z$ along the OD maximum which now is a fracture line, see Fig. 4.22. The angle between the active OP and OD modes is given by $\alpha=\arccos k_{c, o} / k_{c, z}$. This leads to the resonance relation $\vec{k}_{1, z}-\vec{k}_{2, z}-2 \vec{k}_{1, o}=0$, see Fig. 4.22 (a). Interaction terms between OD and OP Fourier modes thus arise through amplitude equations of the form

$$
\begin{aligned}
\partial_{t} A_{1}= & r_{z} A_{1}-\left|A_{1}\right|^{2} A_{1}-2\left|A_{2}\right|^{2} A_{1}-6 \tau|B|^{4}\left(\left|A_{1}\right|^{2} A_{1}+2\left|A_{2}\right|^{2} A_{1}\right) \\
& -4 \tau B^{3} \bar{B}\left(\left|A_{2}\right|^{2} A_{2}+2\left|A_{1}\right|^{2} A_{2}\right)-4 \tau \bar{B}^{3} B A_{1}^{2} \bar{A}_{2}-\tau B^{4} \bar{A}_{1} A_{2}^{2},
\end{aligned}
$$

and a corresponding equation for the mode $A_{2}$.

\subsubsection{Higher feature dimensions}

The inclusion of more feature dimensions into the dynamics can be performed in a similar fashion as the geometric correlations between the maps seem to be universal [68. To illustrate this we used the higher order gradient-type inter-map coupling with three and four cortical maps which are mutually coupled. Whereas in the case of two maps the coupling energy is zero if the two stripe solutions are perpendicular to each other the interactions between more maps potentially leads to a frustration as none of the individual coupling energies vanishes. Using the gradient coupling energy

$$
U=U_{1}+U_{2}+U_{3}=\epsilon_{1}\left|\nabla z \nabla o_{1}\right|^{4}+\epsilon_{2}\left|\nabla z \nabla o_{2}\right|^{4}+\epsilon_{3}\left|\nabla o_{1} \nabla o_{2}\right|^{4}
$$


(a)

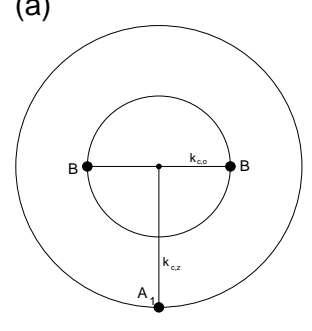

(d)

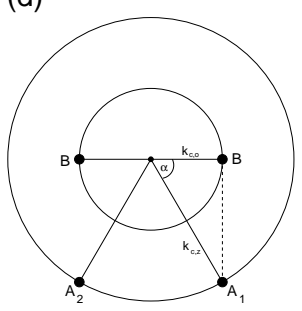

(b)

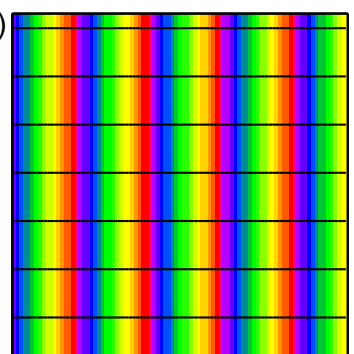

(e)

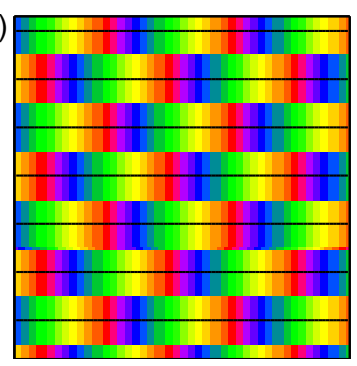

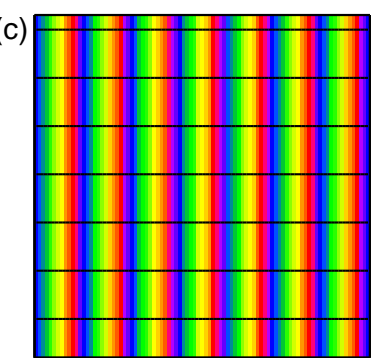

(f)

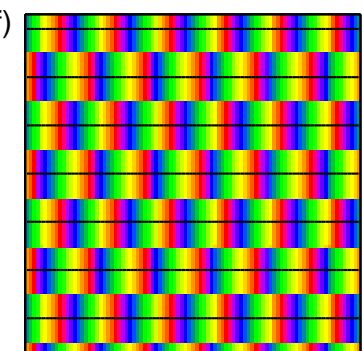

Figure 4.22.: Map interactions with different wavelengths. OD stripes interacting with OP columns where $\Lambda_{o} / \Lambda_{z}>1$, (a-c) $U=\epsilon|\nabla z \nabla o|^{4}$, (d-f) $U=\tau o^{4}|z|^{4}$. (a,d) Illustration of active modes in Fourier space with $k_{c, o}<k_{c, z}, \alpha=\arccos k_{c, o} / k_{c, z}$. Final patterns obtained from numerical simulations, $(\mathbf{b}, \mathbf{e}) \Lambda_{o} / \Lambda_{z}=1.3,(\mathbf{c}, \mathbf{f}) \Lambda_{o} / \Lambda_{z}=2$. Parameters: $\tau=2000, r_{z}=0.05, r_{o}=0.2, T_{f}=5 \cdot 10^{4}, \Gamma_{z}=20,256 \times 256$ mesh.

and no OD bias $(\gamma=0)$ we observe two types of stationary solutions, see Fig. 4.23, Here we use equal coupling strengths $\epsilon_{1}=\epsilon_{2}=\epsilon_{3}=\epsilon$. In case all bifurcation parameters are equal the OP map consists of stripes. Also the two real fields consist of stripes, both perpendicular to the OP stripes i.e.

$$
\begin{aligned}
z(\mathbf{x}) & =A e^{i \vec{k}_{1} \vec{x}} \\
o_{1}(\mathbf{x}) & =2 B_{1} \cos \left(\vec{k}_{2} \vec{x}\right) \\
o_{2}(\mathbf{x}) & =2 B_{2} \cos \left(\vec{k}_{2} \vec{x}+\psi\right), \quad \vec{k}_{1} \cdot \overrightarrow{k_{2}}=0 .
\end{aligned}
$$

The energy in this case is given by $U_{1}=U_{2}=0, U_{3}=\frac{B_{1}^{4} B_{2}^{4} \pi}{16}(18+16 \cos (2 \psi)+\cos (4 \psi))$ which is minimal for $\psi=\pi / 2$, i.e. the energy is minimized by shifting one real field by half of the typical wavelength. When the bifurcation parameter of the OP map is smaller than that of the two real fields we obtain PWC patterns, see Fig. 4.23(b). The pinwheels are arranged such that they are in the center of a square spanned by the two orthogonal real fields and the resulting pinwheel density is $\rho=4$. All intersection angles between iso-orientation lines and borders of the real fields are perpendicular. When extending the system by another real field we observe a similar behavior. Figure $4.23(\mathrm{c}, \mathrm{d})$ shows the stationary states of a complex field coupled to three real fields. In case of equal bifurcation parameters the stationary patterns are OP stripes, perpendicular to the stripe and meandering real solutions. In case the bifurcation parameter of the OP map is smaller than the other bifurcation parameters we again observe pinwheel crystallization. Note, that in this case all pinwheels are located at the border of one of the three real fields. To summarize, pinwheel crystallization is only observed if the OP is 
driven by the real field i.e. if the OP amplitudes are small. In all observed cases the patterns are spatially periodic. 
(a)
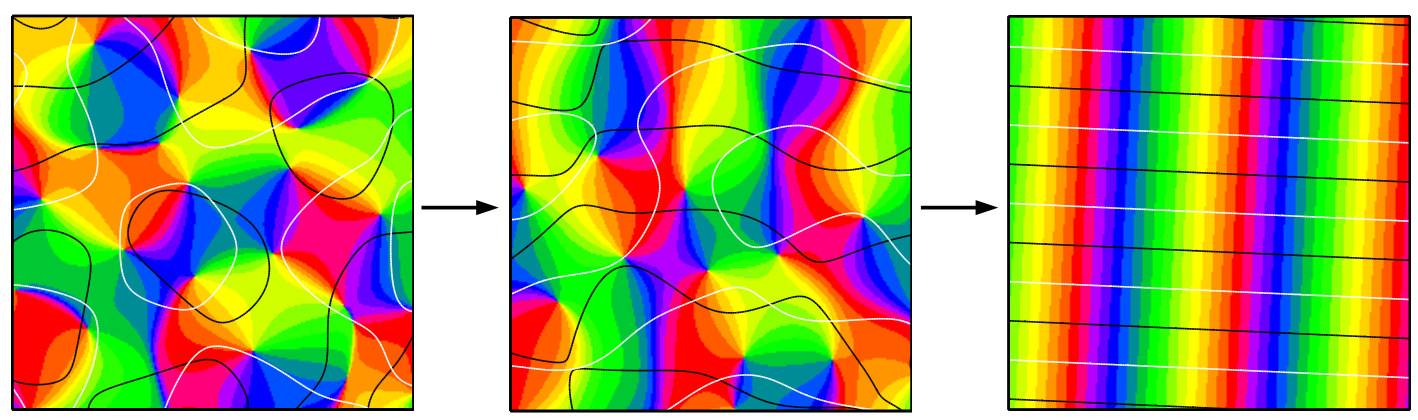

(b)
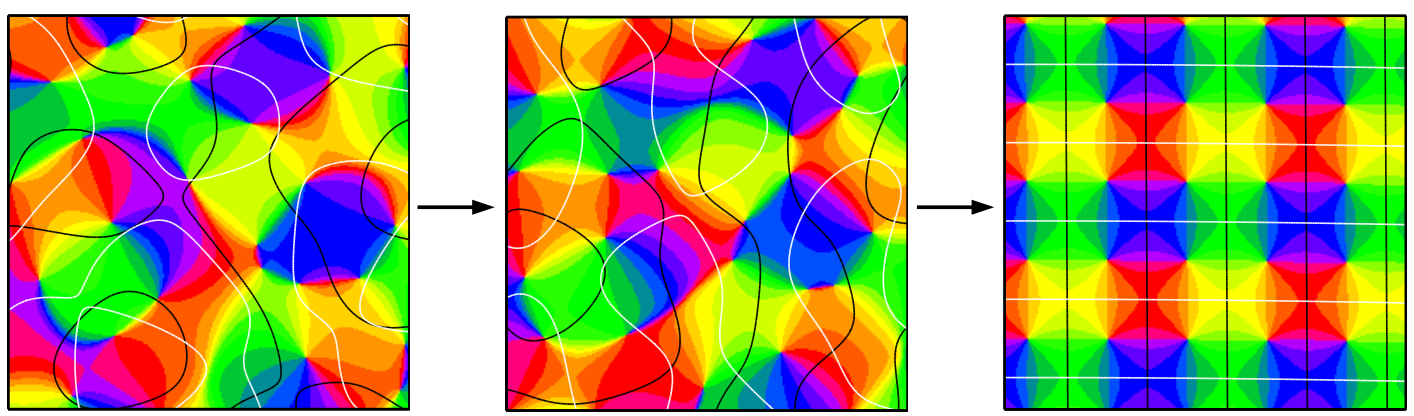

(c)
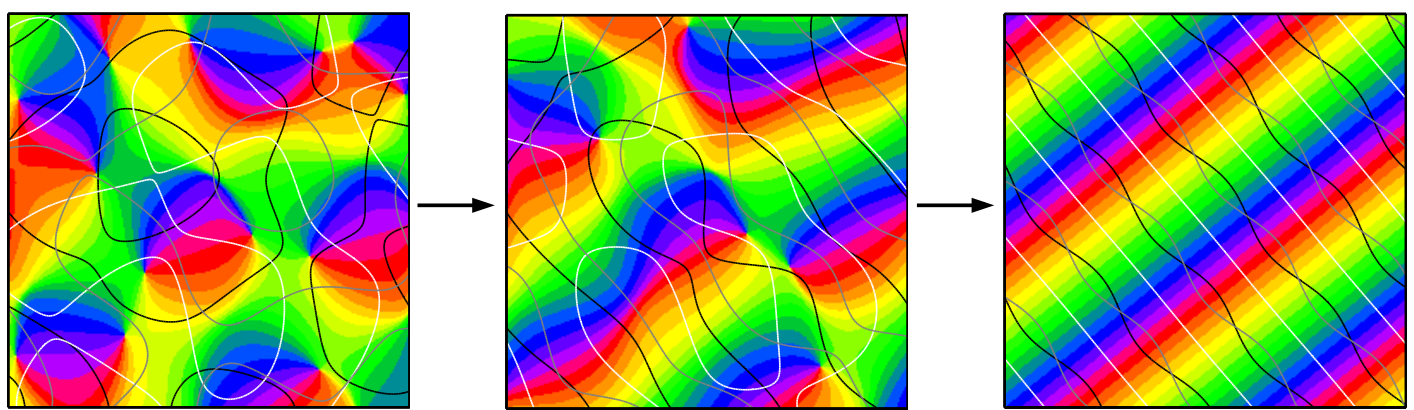

(d)
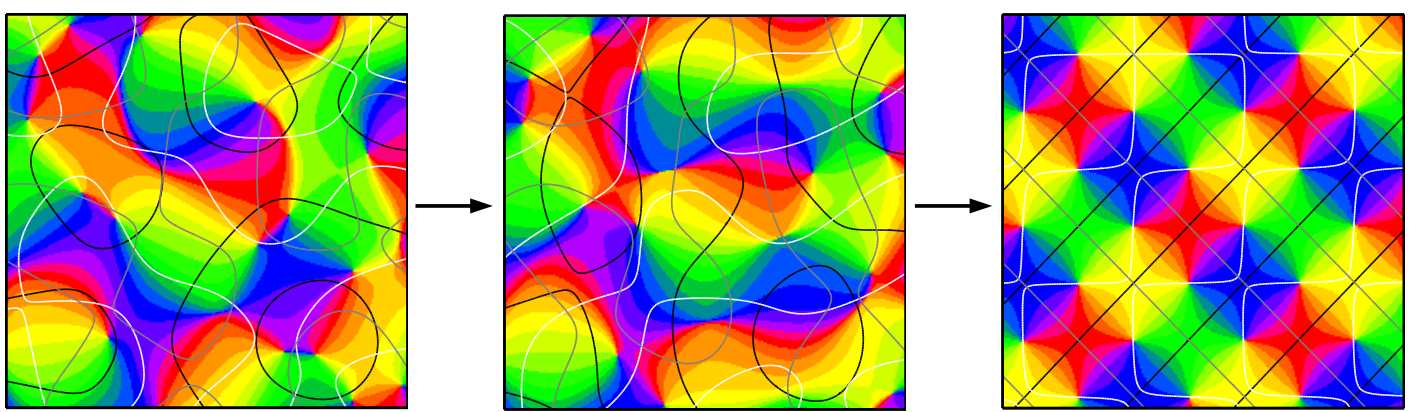

Figure 4.23.: Map interactions in higher feature dimensions. (a,b) Map layout by interactions between three columnar systems $\left(z(\mathbf{x}), o_{1}(\mathbf{x}), o_{2}(\mathbf{x})\right)$. All maps are mutually coupled. Superimposed on the OP map there are the borders of two real fields (black, white). (a) $r_{z}=r_{o_{1}}=r_{o_{2}}=0.1$ (b) $r_{z}=0.01, r_{o_{1}}=r_{o_{2}}=0.1$. (c,d) Interactions with four columnar systems $\left(z(\mathbf{x}), o_{1}(\mathbf{x}), o_{2}(\mathbf{x}, t), o_{3}(\mathbf{x}, t)\right)$. (c) $r_{z}=r_{o_{1}}=r_{o_{2}}=r_{o_{3}}=0.1$. (d) $r_{z}=0.01, r_{o_{1}}=r_{o_{2}}=r_{o_{3}}=0.1$. Superimposed on the OP map there are the borders the of three real fields (black, gray, white). Left panel: initial conditions, middle panel: $T=10$, right panel: $T=T_{f}=5 \cdot 10^{4}$. Parameters in all simulations: $\epsilon=2000, \gamma=0, \Gamma=22$, $128 \times 128$ mesh. 


\subsection{Discussion}

In this chapter we presented a first application of the general model Eq. (3.1) for the coordinated development of different maps in the visual cortex. We demonstrated that OD segregation can stabilize pinwheels even if they are intrinsically unstable in the uncoupled dynamics of the OP map. We identified and analyzed the stability properties of solutions forming a spatially regular layout with pinwheels arranged in a crystalline array. We calculated phase diagrams showing the stability of these pinwheel crystals in dependence on the OD bias and the inter-map coupling strength.

Our results indicate that the overall dominance of one eye is important for the effectiveness of this mechanism. In this case, OD domains form a system of hexagonal patches rather than stripes enabling the capture and stabilization of pinwheels by inter-map coupling. Supporting this notion, visual cortex around the time of early OP development is indeed dominated by one eye and has a pronounced patchy layout of OD domains [56 [58]. Further support for the relevance of this picture comes from experiments in which the OD map was removed artificially resulting in a significantly smoother OP map [33]. We identified three OD solutions: stripes, hexagons, and a constant solution, which are stable depending on the OD bias. All three patterns are found to resemble patterns observed in physiological OD maps. Interestingly, all three types of patterns are found to coexist in the visual cortex of macaque monkeys [56]. For such an OD map our model predicts a systematic variation of the pinwheel density.

To the best of our knowledge we present for the first time an analytical solvable model for the coordinated development of OP and OD maps. We use a dynamical systems approach which allows for a perturbative expansion of the dynamics. Using weakly nonlinear analysis we derived amplitude equations as an approximate description of the dynamics near the pattern formation threshold. We identified fixed points and could study their stability properties using different types of inter-map coupling energies. We found that for the low order version of these energies a strong inter-map coupling can lead to OP map suppression, causing the orientation selectivity of all neurons to vanish. In contrast, the higher order variants of the coupling energies do not lead to map suppression but only influence pattern selection. Moreover, we could identify a limit in which the inter-map coupling becomes unidirectional enabling for the use of the uncoupled OD patterns. To confirm our results and to study the impact of a finite backreaction on the OD map we solved the full field dynamics numerically. In particular, we studied the dynamics of pinwheel crystallization. With the presented analytical approach we were able to show that pinwheel rich solutions correspond to the energetic ground state of the system for large inter-map coupling. This is supported by the fact that pinwheels can actually be created from an initial OP stripe pattern. Pinwheel creation as a result of a pinwheel rich energetic ground state thus serves as a simple test for models of OP development. There is currently only one other model that was shown to be capable of generating pinwheels from pinwheel free initial conditions [49], which will be discussed in the next chapter. 
We studied the impact of inter-map coupling on the geometric relationships between OP and OD maps and showed that from observation of map structures one indeed can learn about the optimized energy. From a symmetry-based analysis we identified a class of energies which reflect the experimentally observed geometric relationships between OD and OP maps 68, 73 75]. We thus studied gradient-type as well as product-type coupling energies. We identified a class of PWC solutions which become stable for large inter-map coupling. This class depends on a single parameter which is specific to the used inter-map coupling energy. This demonstrates that, although pinwheel stabilization is not restricted to a specific choice of the interaction term, each analyzed phase diagram is specific to the coupling energy. In the case of the product-type coupling energies the resulting phase diagrams are relatively complex as stationary solutions and stability borders explicitly depend on the OD bias. In contrast, for the gradient-type coupling energies the bias dependence can be absorbed into the coupling strength and only selects the stationary OD pattern. This leads to a rather simple phase diagram. We identified several biologically implausible OP patterns. In the case of the product-type energies we found orientation scotoma solutions which are selective to only two preferred orientations. In the case of the low order gradient-type energy we found OP patterns containing pinwheels with a topological charge of 1 which have not yet been observed in experiments. We further analyzed the stationary patterns with respect to intersection angles and pinwheel positions. Remarkably, all analyzed PWC solutions show the tendency for iso-orientation lines to intersect OD borders perpendicularly, even in the case of the product-type energy which is not expected per se. However, for the low order versions of the coupling energy the distribution of intersection angles is not continuous. Half of the pinwheels in the analyzed PWC solutions are located at OD extrema, as expected from the used coupling energies. Some pinwheels, however, are located at OD saddle-points. Remarkably, such correlations, which are expected from the gradient-type coupling energies, occur also in the case of the product-type energies. However, correlations between pinwheels and OD saddle-points have not yet been studied quantitatively in experiments.

From the analyzed phase diagrams we conclude that the higher order gradient-type coupling energy best reflects the map layouts and their correlations in the visual cortex. As we will see in the next chapter, a clear distinction between the energies with respect to the geometric relationships can be made only when considering spatially aperiodic map layouts. We restricted our analysis to four representative examples of coupling energies. The general structure of the amplitude equations is universal and only the coupling coefficients change when changing the coupling energy. We therefore expect that also for other coupling energies, respecting the proposed set of symmetries, PWC solutions become stable for large inter-map coupling.

In this chapter we showed that the presented model offers a solution to the problem of pinwheel stability. To solve this problem, the influence of OD segregation on OP maps previously had been studied in a series of models such as elastic net models [18, 27 [31], self-organizing map models [24,32 35], spin-like Hamiltonian models [20], spectral filter models [37], correlation based models [36, and evolving field models [38]. One should note, however, that the previous models mentioned above are a numerical approach. Whether the obtained solutions 
are attractors or just transient states and the solutions further develop towards pinwheel free solutions remains unclear. In particular, it is in principle conceivable that their solutions will converge to crystalline arrangements in long simulations. Moreover, in many previous models a continuous variation of the inter-map coupling strength is not possible which makes it hard to disentangle the contribution of each map.

The presented model questions the widely held view that OD stripes are capable of stabilizing pinwheels. Our analysis shows that OD stripes are indeed not able to stabilize pinwheels, a result that appears to be independent of the specific type of map interaction. Several other theoretical studies, using numerical simulations [18, 38, 99], indicate that a more banded OD pattern leads to a less pinwheel rich OP map.

We presented a thorough characterization of the stable OP and OD patterns. However, the pinwheel crystals we obtained in our model, although beautiful and easy to characterize, in several aspects deviate from experimentally obtained maps. All PWC solutions have a large pinwheel density of about 3.5 or even 5.2 pinwheels per $\Lambda^{2}$. Densities obtained from experiments, although broadly scattered, are in the range of 3 pinwheels per $\Lambda^{2}[16]$. In contrast to the large variability of map layouts in experimentally observed maps, the pinwheel crystals show a regular and stereotyped structure. The reason for this might be neglected biological constraints in our model such as realistic boundary conditions or biological noise in the system. Another aspect is the developmental stage which is represented by the maps of our model. PWC solutions represent attractor states and thus correspond to late stages of development potentially only comparable to the late adult pattern. One might therefore expect that during the development a further crystallization of the pattern, along with an increase in the pinwheel density takes place. Pinwheel crystals have been previously reported in several abstract [17,19] as well as feature based models [100]. Remarkably, when modeling receptive field development with synaptic long-range connections the resulting OP map shows a striking similarity to the hexagonal PWC presented in this chapter [101.

We showed that the presented framework can be generalized to include any additional columnar system and thus is not restricted to interactions among OD and OP maps. A reason to consider other visual cortical maps originates from the finding that the removal of the OD map in experiments does not completely destabilize pinwheels [33. Moreover, in tree shrews, animals which lack OD columns, the OP maps contain pinwheels [39]. This might reflect the influence of additional columnar systems like spatial frequency columns that are expected to interact with the OP map in a similar fashion as OD columns 68]. To better account for the non-crystalline layout of experimentally obtained maps we also extended the map interaction model to higher dimensions. In numerical simulations we illustrated inter-map coupling with three and four columnar systems. Although in this case pinwheel stabilization is possible even without an OD bias, the resulting stationary OP patterns are either stripes or PWC solutions. Higher feature dimensions are often treated with dimension reduction models [24,102]. A dependence of the number of feature maps on the stabilization of pinwheels was observed for instance in a Kohonen model [100]. We did not consider an interaction with the retinotopic map. Compared to other feature maps, the retinotopic map seems to be exceptional 
concerning the geometric relationships. It has been observed that the correlations between OP and retinotopic map are such that high gradient regions do not avoid each other [103]. Such correlations cannot be easily treated with dimension reduction models, see [104]. Remarkably, in our model we identified coupling terms that could account for an attraction of high gradient regions. Such terms contain the gradient of only one field and can thus be considered as a mixture of the gradient and the product-type energy.

Our analysis conclusively demonstrates that OD segregation can stabilize pinwheel crystals even if they are intrinsically unstable in the uncoupled dynamics of the OP map, raising the possibility that inter-map coupling is the mechanism of pinwheel stabilization in the visual cortex. In the next chapter, we study an alternative hypothesis for the origin of pinwheel stabilization. Neurons in the visual cortex form extensive connections horizontal to the cortical surface linking different orientation columns [39 48]. It is possible to include these long-range connections in our dynamics by adding non-local interaction terms. It has been shown [49,50] that with increasing spatial extend of these interactions the layout of maps becomes more irregular. 


\section{Shaping the Layout of OD Maps by Inter-Map Coupling}

\subsection{Introduction}

We have seen in the previous chapter that map interactions can lead to a stabilization of pinwheel rich OP patterns. The resulting OP maps, however, are pinwheel crystals and thus spatially periodic. In this chapter we add a source of irregularity by studying a second hypothesis of pinwheel stabilization. It has been demonstrated that the inclusion of long-range interactions in the dynamics leads to stable pinwheel rich OP patterns [49, 50]. Compared to the pinwheel crystals these solutions have a spatially irregular layout. In this chapter we discuss the consequences of long-range interactions in the coupled model.

In particular, we show that the spatially irregular OP layout can be transferred onto the OD map. Similar to the previous chapter we identify a limit in which the backreaction on the dynamics of the OP map can be neglected. We identify an analytically tractable class of stationary solutions and provide their stability criteria. When using identical wavelengths for both maps we show that the OD pattern assumes a patchy layout which resembles the patterns found in cats. For the stable solutions we quantify the geometric relationships showing, for instance, that these relationships are specific to the used type of coupling energy. We further study the impact of the backreaction on the OP map demonstrating that the pinwheel rich, aperiodic solutions are preserved even for a considerable amount of backreaction. We give a potential explanation of the OD layout differences in cats and macaque monkeys. When introducing a detuning in the average wavelength between OP and OD maps as found in macaque monkeys we show that the resulting OD layout is stripe-like rather than patchy, resembling the OD layout found in monkeys.

The coupled dynamics of OD and OP maps we consider in this chapter is given by

$$
\begin{aligned}
& \partial_{t} z(\mathbf{x}, t)=\hat{L}_{z} z(\mathbf{x}, t)-N_{3}[z, z, \bar{z}]-\frac{\delta U}{\delta \bar{z}} \\
& \partial_{t} o(\mathbf{x}, t)=\hat{L}_{o} o(\mathbf{x}, t)-o(\mathbf{x}, t)^{3}-\frac{\delta U}{\delta o} .
\end{aligned}
$$

The cubic nonlinearity $N_{3}[z, z, \bar{z}]$, introduced in Section [3.1, contains local and non-local contributions given by

$$
\begin{aligned}
N_{3}[z, z, \bar{z}]= & (g-1)|z(x)|^{2} z(x)+ \\
& \frac{2-g}{2 \pi \sigma^{2}} \int d^{2} y e^{-|x-y|^{2} / 2 \sigma^{2}}\left(z(x)|z(y)|^{2}+\frac{1}{2} \bar{z}(x) z(y)^{2}\right) .
\end{aligned}
$$


The dynamics can be derived from the energy functional

$$
\begin{aligned}
E[z, o]= & -\int d^{2} x\left(\bar{z}(\mathbf{x}) \hat{L}_{z} z(\mathbf{x})+\frac{1}{2} o(\mathbf{x}) \hat{L}_{o} o(\mathbf{x})+\frac{1}{2}(1-g)|z(\mathbf{x})|^{4}-\frac{1}{4} o(\mathbf{x})^{4}\right) \\
& +\frac{2-g}{4 \pi \sigma^{2}} \int d^{2} x \int d^{2} y\left(|z(\mathbf{x})|^{2}|z(\mathbf{y})|^{2}+\frac{1}{2} z((y))^{2} \bar{z}^{2}(\mathbf{x})\right) e^{-|\mathbf{x}-\mathbf{y}|^{2} /\left(2 \sigma^{2}\right)} \\
& +\int d^{2} x U
\end{aligned}
$$

Non-local interactions are introduced only for the OP part of the dynamics. Experiments suggest that long-range horizontal connections are sensitive to the preferred orientation of the neurons which are interconnected while their correlations to OD is less clear, see Section 2.3 , Compared to the previous chapter the dynamics is invariant under the inversion symmetry $o(\mathbf{x}) \rightarrow-o(\mathbf{x})$ and the uncoupled OD attractors are thus stripes for $r_{o}>0$. As in the previous chapter we study the four representative examples of coupling energies $U$ given in Eq. (3.23).

\subsection{Permutation symmetry}

When we rewrite the nonlinearity Eq. (5.2) in the following form

$$
\begin{aligned}
N[u, v, w]= & (g-1) u(\mathbf{x}) v(\mathbf{x}) w(\mathbf{x}) \\
& +\frac{2-g}{4 \pi \sigma^{2}} \int d^{2} y e^{-|\mathbf{x}-\mathbf{y}|^{2} /\left(2 \sigma^{2}\right)}(u(\mathbf{x}) v(\mathbf{y}) w(\mathbf{y})+ \\
& v(\mathbf{x}) w(\mathbf{y}) u(\mathbf{y})+w(\mathbf{x}) u(\mathbf{y}) v(\mathbf{y}))
\end{aligned}
$$

we see that the nonlinearity is invariant under a cyclic permutation of the fields i.e.

$$
N[u, v, w]=N[v, w, u] .
$$

This permutation symmetry has far reaching consequences on the attractor states of the dynamics. In the following we give a brief review of the uncoupled OP dynamics with permutation symmetry. Consequences of the presence or absence of this symmetry are also discussed in Chapter 6 and 7 .

\subsection{The uncoupled dynamics}

Without inter-map coupling the dynamics for the OP map is studied in [49,50]. In this section we briefly review the main results of this work. In case of the uncoupled dynamics we can use the low order amplitude equations derived in Section 4.3. With the inclusion of the non-local 
interaction terms Eq. (5.2) the coupling coefficients, see Eq. (4.59), are given by

$$
\begin{aligned}
g_{i j} & =g+(2-g)\left(e^{-\frac{1}{2} \sigma^{2}\left(\vec{k}_{i}-\vec{k}_{j}\right)^{2}}+e^{-\frac{1}{2} \sigma^{2}\left(\vec{k}_{i}+\vec{k}_{j}\right)^{2}}\right) \\
g_{i i} & =1+\frac{1}{2}(2-g) e^{-2 \sigma^{2}} \\
f_{i j} & =g+(2-g)\left(e^{-\frac{1}{2} \sigma^{2}\left(\vec{k}_{i}-\vec{k}_{j}\right)^{2}}+e^{-\frac{1}{2} \sigma^{2}\left(\vec{k}_{i}+\vec{k}_{j}\right)^{2}}\right) \\
f_{i i} & =0 .
\end{aligned}
$$

If the directions of the wave vectors $\vec{k}_{j}=\left(\cos \alpha_{j}, \sin \alpha_{j}\right) k_{c, z}$ are represented by angles $\alpha_{j}$ then the coefficients $g_{i j}$ and $f_{i j}$ are functions only of the angle $\alpha=\left|\alpha_{i}-\alpha_{j}\right|$ between the wavevectors $\vec{k}_{i}$ and $\vec{k}_{j}$. The coefficients can be obtained from the continuous functions

$$
\begin{aligned}
& g(\alpha)=e^{-\imath \vec{k}_{0} \vec{x}}\left(N_{3}\left[e^{\imath \vec{k}_{0} \vec{x}}, e^{\imath h(\alpha) \vec{x}}, e^{-\imath h(\alpha) \vec{x}}\right]+N_{3}\left[e^{\imath h(\alpha) \vec{x}}, e^{\imath \vec{k}_{0} \vec{x}}, e^{-\imath h(\alpha) \vec{x}}\right]\right) \\
& f(\alpha)=e^{-\imath \vec{k}_{0} \vec{x}}\left(N_{3}\left[e^{\imath h(\alpha) \vec{x}}, e^{-\imath h(\alpha) \vec{x}}, e^{\imath \vec{k}_{0} \vec{x}}\right]+N_{3}\left[e^{-\imath h(\alpha) \vec{x}}, e^{\imath h(\alpha) \vec{x}}, e^{\imath \vec{k}_{0} \vec{x}}\right]\right),
\end{aligned}
$$

with $\vec{k}_{0}=(1,0) k_{c, z}$ and $h(\alpha)=(\cos \alpha, \sin \alpha) k_{c, z}$. The coefficients are obtained as $g_{i j}=$ $g\left(\left|\alpha_{i}-\alpha_{j}\right|\right), g_{i i}=g(0) / 2, g_{i j^{-}}=g\left(\left|\alpha_{i}-\alpha_{j}+\pi\right|\right)$ and $f_{i j}=f\left(\left|\alpha_{i}-\alpha_{j}\right|\right)$. In case of the coupling coefficients Eq. (5.6) the coupling function is given by

$$
\begin{aligned}
& g(\alpha)=g+(2-g)\left(e^{-\sigma^{2}(1+\cos \alpha)}+e^{-\sigma^{2}(1-\cos \alpha)}\right) \\
& f(\alpha)=g+(2-g)\left(e^{-\sigma^{2}(1-1 \cos \alpha)}+e^{-\sigma^{2}(1+\cos \alpha)}\right) .
\end{aligned}
$$

Whereas $f(\alpha)$ is $\pi$-periodic, $g(\alpha)$ is $2 \pi$-periodic in general. The permutation symmetry Eq. (5.5), however, implies $g(\alpha+\pi)=g(\alpha)$.

Close to the bifurcation point a large class of attractors to the uncoupled OP dynamics is given by

$$
z(\mathbf{x})=\sum_{j}^{n_{z}} \mathcal{A}_{j} e^{\imath \phi_{j}} e^{\imath l_{j} \vec{k}_{j} \vec{x}}
$$

The binary variable $l_{j}= \pm 1$ determines whether the mode with wavevector $\vec{k}_{j}$ or $-\vec{k}_{j}$ is active. For this class of solutions opposite modes are suppressed i.e. $A_{j^{-}}=0$. Planforms of this type are therefore called Essentially Complex Planforms (ECP). For an ECP the field $z(\mathbf{x})$ cannot become real and thus the OP map is selective to all possible stimulus orientations. The phases of the active modes $\phi_{j}$ are arbitrary and independent of the mode configuration $l_{j}$. Due to permutation symmetry $g_{i j}$ is a circulant matrix $g_{i j}=g_{(j-i) \bmod n}$. Since $\sum_{j} g_{i j}$ is in this case independent of the index $i$ the stationary solutions are uniform and given by

$$
\mathcal{A}_{i}=\mathcal{A}=\sqrt{\frac{r_{z}}{\sum_{j} g_{i j}}} .
$$


(a)

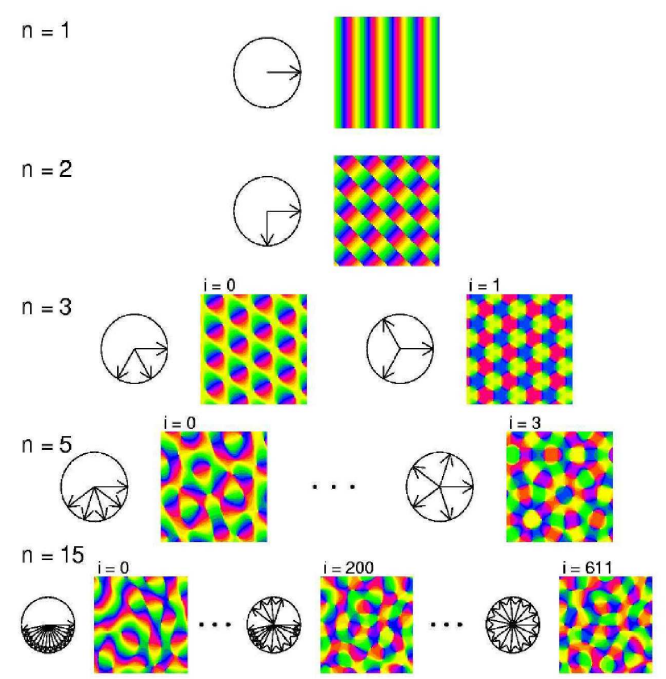

(b)

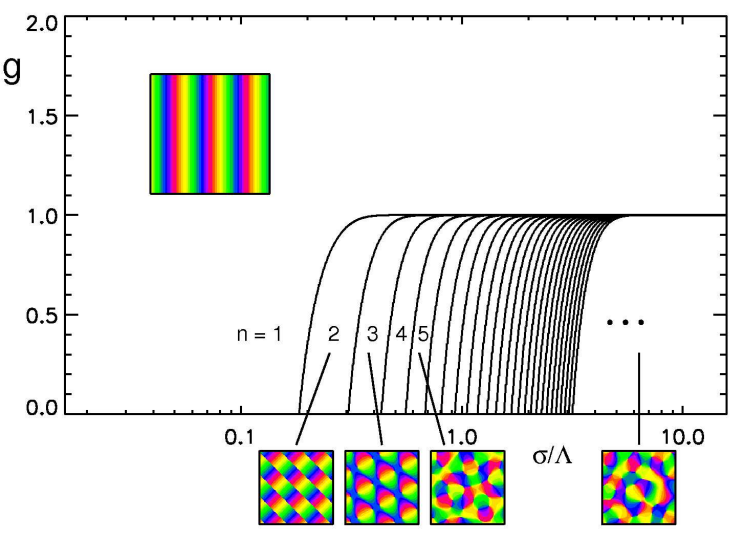

Figure 5.1.: Phase diagram of the uncoupled OP dynamics. (a) Essentially complex planforms with different numbers $n_{z}=n=1,2,3,5,15$ of active modes. The diagrams to the left of each pattern display the position of the wavevectors of active modes on the critical circle. For $n=3$, there are two patterns; for $n=5$, there are four, and for $n=15$, there are 612 different patterns. (b) Phase diagram of the uncoupled case. Shown are the regions where a essential complex planform with $n$ modes has the minimum energy. If non-local interactions are dominant $(g<1)$ and long-ranging ( $\sigma$ large compared to $\Lambda$ ), quasiperiodic planforms are selected. Reproduced from [49].

For a given $n_{z}$ there are $2^{n_{z}}$ possible ECP configurations, however, many of which can be transferred into each other by a rotation or reflection thus defining equivalence classes. The actual number of distinct classes is smaller but nevertheless grows exponentially with $n_{z}$. Their phase diagram is shown for the two model parameters $g$ and $\sigma / \Lambda$ in Fig. 5.1(b). For $g>1$ only $n_{z}=1$ solutions are energetically preferred while for $g<1$ and $\sigma / \Lambda$ large enough solutions with more modes $n_{z}>1$ are energetically preferred. The different ECP solutions substantially vary in their pinwheel density. In the following we denote LDP as the low pinwheel density planform given by $l_{j}=1, \forall j$ and HDP as the high pinwheel density planform given by $l_{j}=1$ for $j$ even and $l_{j}=-1$ for $j$ odd. Note, the HDP configuration may not be the one with the maximum pinwheel density but has a pinwheel density larger than $\rho=\pi$. How the pinwheel density varies with the different planforms is detailed in Chapter 6 . The model has a vast number of multistable solutions. For a given $n_{z}$ there is a multistability of different ECP solutions, see Fig. 5.1(a). As shown in [49,50] permutation symmetry ensures that for a given $n_{z}$ all ECP solutions share the same energy and stability properties. Realistic patterns are obtained for $g<1$ and $\sigma \gg \Lambda$, see Fig. 5.1(b). In the case of $n_{z} \geq 4$ the corresponding OP map is a spatially quasiperiodic pattern becoming more and more irregular with increasing $n_{z}$. For intermediate and large $n_{z}$ these patterns resemble those obtained in experiments. 
Without inter-map coupling the OD maps have a regular stripe layout given by

$$
o(\mathbf{x})=2 \sqrt{\frac{r_{o}}{3}} \cos \left({\overrightarrow{k^{\prime}}}_{1} \vec{x}+\psi\right) .
$$

This layout does not resemble the patchy layout observed in cats. Even the more stripe-like layouts found in monkeys and humans where OD bands meander, occasionally branching and terminating, are much more irregular than OD stripes.

In the following we study how inter-map coupling influences the layout of OD and OP maps. In particular, we are interested in a limit in which we can neglect the backreaction on the OP map. Besides a substantial simplification this limit allows us to use the uncoupled OP attractors which, for large $n_{z}$, resembles the OP layout found in physiological maps. We mainly concentrate on planforms with $n_{z} \geq 4$ because in contrast to the last chapter these solutions have a spatially irregular layout.

\subsection{Low order coupling energies}

In this section we study the impact of inter-map coupling on the layout of OD maps using the low order inter-map coupling energy terms in Eq. (3.23). We show that, similar to the case of PWC solutions, there is a suppression of OD leading to a completely unselective OD map. Close to the bifurcation point $r_{z}=0, r_{o}=0$ stationary solutions to the dynamics Eq. (5.1) are calculated using weakly nonlinear analysis. The general derivation of coupled amplitude equations up to seventh order is given in Section 4.3. In contrast to the previous chapter we want to neglect the backreaction onto the OP map and thus assume $r_{o} \ll r_{z}$. The Fourier components of the emerging pattern are located on the two critical circles with the wavevectors $\vec{k}_{j}=\left(\cos \alpha_{j}, \sin \alpha_{j}\right) k_{c, z}$ and ${\overrightarrow{k^{\prime}}}_{j}=\left(\cos \alpha_{j}+\alpha, \sin \alpha_{j}+\alpha\right) k_{c, o}$. We expand both fields in a sum of these active modes

$$
\begin{aligned}
& z(\mathbf{x}, t)=\sum_{j}^{n_{z}} A_{j}(t) e^{\imath l_{j} \vec{k}_{j} \vec{x}}+\sum_{j}^{n_{z}} A_{j-}(t) e^{-\imath l_{j} \vec{k}_{j} \vec{x}} \\
& o(\mathbf{x}, t)=\sum_{j}^{n_{o}} B_{j}(t) e^{\imath \vec{k}_{j}^{\prime} \vec{x}}+\sum_{j}^{n_{o}} \bar{B}_{j}(t) e^{-\imath \overrightarrow{k_{j}^{\prime}} \vec{x}}
\end{aligned}
$$

with the complex amplitudes $A_{j}=\mathcal{A}_{j} e^{\imath \phi_{j}}$ and $B_{j}=\mathcal{B}_{j} e^{\imath \psi_{j}}$.

To illustrate the suppression of the pattern, observed for the low order inter-map coupling energies already in the last chapter, we consider the coupled amplitude equations in the case of $n_{o}=n_{z}=4$. The dynamics for the OD amplitudes in this case is given by

$$
\partial_{t} B_{i}=r_{o} B_{i}-\sum_{j}^{4} \widetilde{g}_{i j}\left|B_{j}\right|^{2} B_{i}-\sum_{j}^{4} h_{i j}\left|A_{j}\right|^{2} B_{i}-\sum_{j \neq i}^{4} \widetilde{h}_{i j} A_{i} \bar{A}_{j} B_{j},
$$



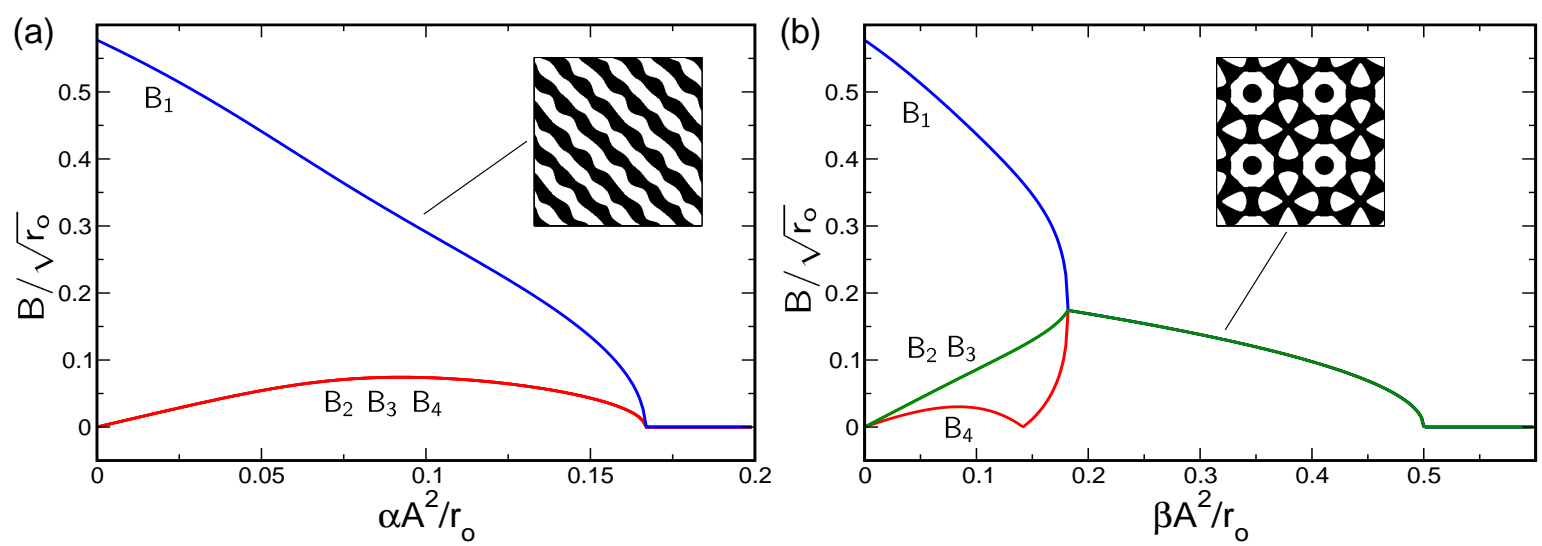

Figure 5.2.: Stationary OD amplitudes for low order coupling energies and $n=4$ planforms, $r_{o}=0.1$. (a) $U=\alpha o^{2}|z|^{2}$, stripe-like solutions, (b) $U=\beta|\nabla o \nabla z|^{2}$, transition towards a uniform solution. Insets: Sign of $o(\mathbf{x})$.

with $\widetilde{g}_{i i}=3, \widetilde{g}_{i j}=6, h_{i j}=2 \alpha+2 \beta \cos ((i-j) \pi / 4)^{2}, \widetilde{h}_{i j}=2 \alpha+\beta\left(1+\cos ((i-j) \pi / 4)^{2}\right)$. We set $A_{j}=\mathcal{A}_{j} e^{\imath \phi_{j}}, B_{j}=\mathcal{B}_{j} e^{\imath \psi_{j}}$ and split these equations into the amplitude and phase parts. In case of uniform amplitudes $\mathcal{A}_{j}=\mathcal{A}$ the dynamics of the OD amplitudes is given by

$$
\partial_{t} \mathcal{B}_{i}=r_{o} \mathcal{B}_{i}-\sum_{j}^{4} \widetilde{g}_{i j} \mathcal{B}_{j}^{2} \mathcal{B}_{i}-\sum_{j}^{4} \mathcal{A}^{2} \mathcal{B}_{j} h_{i j}^{(u)} \cos \left(\phi_{i}-\phi_{j}-\psi_{i}+\psi_{j}\right)
$$

with $h_{i i}^{(u)}=\sum_{j} h_{i j}=8 \alpha+4 \beta, h_{i j}^{(u)}=\widetilde{h}_{i j}$. Stationary amplitudes for the gradient and product-type inter-map couplings are shown in Fig. [5.2. In case of the product-type coupling we observe, for low inter-map coupling, a stripe-like OD pattern with one dominant mode $\left(\mathcal{B}_{1}\right)$, see Fig. [5.2(a). Increasing the inter-map coupling leads to a suppression of the dominant modes while the remaining modes stay small and finally are also suppressed. Lastly at $\alpha \mathcal{A}^{2} / r_{o} \approx 0.17$ all modes are zero and only the trivial solution $B_{j}=0$ is stable. In case of the gradient-type inter-map coupling we observe a transition towards a uniform solution with the phase relations $\psi_{j}=j \pi+\phi_{j}$. The amplitude for this uniform solution is given by

$$
\mathcal{B}=\sqrt{\frac{r_{o}-\mathcal{A}^{2}(6 \alpha+2 \beta)}{21}} .
$$

At $\beta \mathcal{A}^{2} / r_{o} \approx 0.2$ the uniform solution becomes stable. Increasing the inter-map coupling further again leads to a suppression of the amplitude $\mathcal{B}$. Finally, at $\beta \mathcal{A}^{2} / r_{o}=0.5$ the pattern solution losses its stability and only the trivial solution is stable.

This section demonstrates that the low order inter-map coupling energies can lead to a complete suppression of OD, similar to OP selectivity suppression discussed in Section 4.4. There is thus no limit $r_{o} / r_{z} \rightarrow 0$ in which we could neglect the backreaction onto the OP map. 


\subsection{Higher order coupling energies}

As the low order coupling energies not only affect pattern selection but also lead to a suppression of the pattern solution we also discuss the higher order coupling energies. As seen in Section 4.3, this leads to interaction terms which enter at seventh order in the amplitude expansion. Here, we discuss two possible configurations of the OD and OP Fourier modes. We first discuss the simplest case in which the wavevectors of the modes $A_{j}$ are independent of the wavevectors of the modes $B_{j}$. This configuration is illustrated in Fig. [5.3(a) by red lines. The amplitude equations in this case are of the form

$$
\begin{aligned}
\partial_{t} A_{i}= & r_{z} A_{i}-\sum_{j}^{n_{z}} g_{i j}\left|A_{j}\right|^{2} A_{i}-\sum_{j}^{n_{z}} f_{i j} A_{j} A_{j^{-}} \bar{A}_{i^{-}} \\
& -\sum_{u, v}^{n_{o}} \sum_{j}^{n_{z}}\left(h_{i j u v}\left|B_{u}\right|^{2}\left|B_{v}\right|^{2}\left|A_{j}\right|^{2} A_{i}+\eta_{i j u v}\left|B_{u}\right|^{2}\left|B_{v}\right|^{2} A_{j} A_{j^{-}} \bar{A}_{i^{-}}\right) \\
\partial_{t} B_{i}= & r_{o} B_{i}-\sum_{j}^{n_{o}} \widetilde{g}_{i j}\left|B_{j}\right|^{2} B_{i} \\
& -\sum_{u, v}^{n_{z}} \sum_{j}^{n_{o}}\left(h_{i j u v}\left|A_{u}\right|^{2}\left|A_{v}\right|^{2}\left|B_{j}\right|^{2} B_{i}+\eta_{i j u v} A_{u} A_{u^{-}} \bar{A}_{v} \bar{A}_{v^{-}}\left|B_{j}\right|^{2} B_{i}\right) .
\end{aligned}
$$

The coupling coefficients $h_{i j u v}$ are given in Appendix A.3. Note, that the phases of the modes $A_{j}$ are unrelated to the phases of the modes $B_{j}$. In the limit $r_{o} \ll r_{z}$ the backreaction onto the dynamics of $A_{i}$ can be neglected and we get an effective cubic interaction term

$$
\partial_{t} B_{i}=r_{o} B_{i}-\sum_{j}^{n_{o}}\left(\widetilde{g}_{i j}+h_{i j}\right)\left|B_{j}\right|^{2} B_{i}, \quad h_{i j}=\sum_{u, v}^{n_{z}}\left(h_{i j u v}\left|A_{u}\right|^{2}\left|A_{v}\right|^{2}+\eta_{i j u v} A_{u} A_{u^{-}} \bar{A}_{v} \bar{A}_{v^{-}}\right) .
$$

In the following we show that the amplitude equations Eq. (5.17) do not lead to stable and spatially irregular OD patterns when coupled to OP solutions with amplitudes given in Eq. (5.10). In the case of $A_{i}=\mathcal{A}, A_{i^{-}}=0$ and $n_{z} \geq 3$ the coupling coefficients are given by

$$
h_{i j}=\left\{\begin{array}{cc}
\epsilon k_{c, z} k_{c, o} \mathcal{A}^{4} 6\left(n_{z}^{2}-3 / 4 n_{z}\right)+\tau \mathcal{A}^{4} 6\left(2 n_{z}^{2}-n_{z}\right) & i=j \\
\epsilon k_{c, z} k_{c, o} \mathcal{A}^{4} 4\left(n_{z}^{2}-3 / 4 n_{z}\right)\left(2+\cos 2 \alpha_{i j}\right)+\tau \mathcal{A}^{4} 12\left(2 n_{z}^{2}-n_{z}\right) & i \neq j
\end{array}\right.
$$

This leads to the coupling function

$$
h(\alpha)=\epsilon k_{c, z} k_{c, o} \mathcal{A}^{4} 4\left(n_{z}^{2}-3 / 4 n_{z}\right)(2+\cos 2 \alpha)+\tau \mathcal{A}^{4} 12\left(2 n_{z}^{2}-n_{z}\right),
$$

with $h_{i j}=h\left(\alpha_{i}-\alpha_{j}\right), h_{i i}=h(0) / 2$. The shape of this coupling function is shown in Fig. [5.3(b). In case of the gradient-type inter-map coupling the coupling function depends on the size of the critical circles $k_{c, o}$ and $k_{c, z}$. As not stated otherwise we assume in the following $k_{c, o}=k_{c, z}=1$ which corresponds to the situation found in cats. A detuning of wavelength $k_{c, o} \neq k_{c, z}$ will be discussed in Section 5.9.3. 

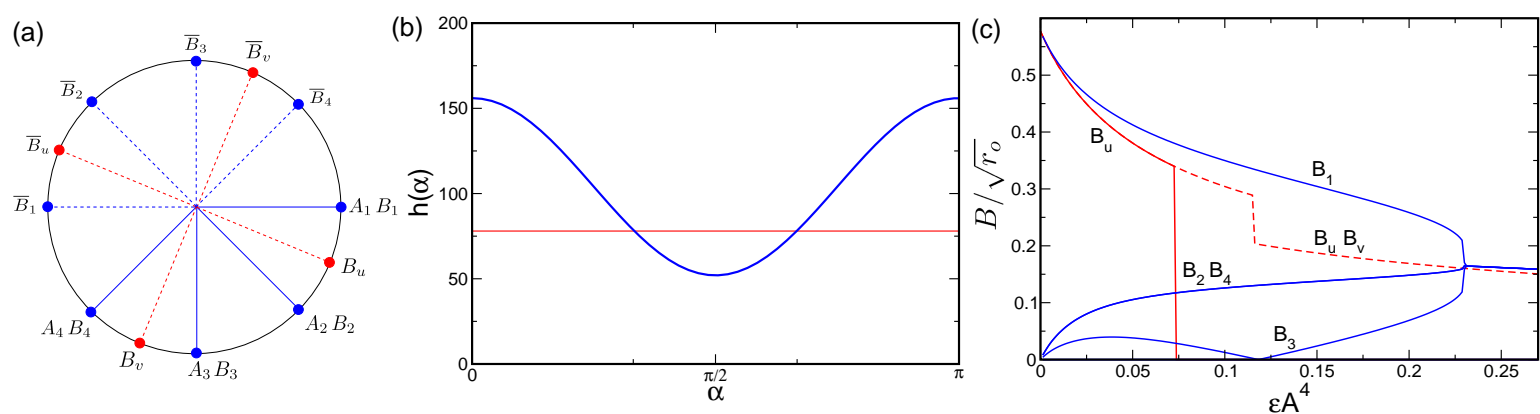

Figure 5.3.: Configuration dependence of OD solutions. (a) Configuration of Fourier modes, Red: $n_{z}=4, n_{o}=2$, non-zero angle between the OP and OD modes. Blue: $n_{z}=n_{o}=4$, superimposed OP and OD Fourier modes. (b) Coupling function, Eq. (5.19). $k_{c, z}=k_{c, o}=1, \epsilon \mathcal{A}^{4}=1, \tau=0, n_{z}=4$. Red line: $h(0) / 2$. (c) Stationary OD amplitudes for both configurations. Red dashed line: unstable solution.

The effective coupling matrix $G_{i j}=\widetilde{g}_{i j}+h_{i j}$ is a circulant matrix of dimension $n_{o}$. As the eigenvalues of circulant matrices are given by $\omega_{l}=\sum_{j} G_{0, j} \cos 2 \pi \frac{j l}{n_{o}}$, (see for instance [105]) the criterion for the stability of $n_{o}>1$ solutions is given by $G_{i j}<G_{i i}$. For the stability of $n_{o}=2$ solutions this leads to $\epsilon \mathcal{A}^{4}>\frac{3}{2\left(n_{z}^{2}-3 / 4 n_{z}\right)}+\frac{3 \tau \mathcal{A}^{4}\left(4-8 n_{z}\right)}{\left(3-4 n_{z}\right)}$ while $n_{o}>2$ solutions are not stable at all. In case of $n_{z}=4, \tau=0$ this leads to $\mathcal{A}^{4} \epsilon \approx 0.12$. When we take a potential growth of modes at the position of the modes $A_{j}$ into account we observe that the OD stripe solution becomes unstable already at $\epsilon \mathcal{A}^{4} \approx 0.07$ where new modes $B_{i}$ grow. This situation is illustrated for $n_{z}=4$ in Fig. [5.3(c). This shows that the configuration depicted in blue in Fig. [5.3(a) is the one which is preferred by inter-map coupling. We will see in the following that this configuration can lead to a spatially irregular OD layout.

\subsubsection{Phase equations}

In the case of modes situated above another mode $k_{i, z}=k_{i, o}$ additional contributions to the amplitude equations appear. These new terms couple the phases $\phi_{j}, \psi_{j}$ of the two patterns. These terms make the $n_{o}<n_{z}$ solutions unstable and we can set $n_{z}=n_{o}=n$. The complete OD dynamics is then given by

$$
\begin{aligned}
\partial_{t} B_{i}= & r_{o} B_{i}-\sum_{j}^{n}\left(\widetilde{g}_{i j}+h_{i j}\right)\left|B_{j}\right|^{2} B_{i}-\sum_{j, l, k}^{n} l_{i j l k} A_{i} A_{j} \bar{A}_{l} \bar{A}_{k} B_{l} B_{k} \bar{B}_{j} \\
& -\sum_{j, l, k}^{n} \lambda_{i j l k} A_{j} \bar{A}_{l}\left|A_{k}\right|^{2} \bar{B}_{j} B_{l} B_{i}-\sum_{j, l, k}^{n} \rho_{i j l k} A_{i} \bar{A}_{l}\left|A_{k}\right|^{2}\left|B_{j}\right|^{2} B_{l},
\end{aligned}
$$

and similar terms for the dynamics of $A_{i}$ and $A_{i^{-}}$, see Chapter 4 . The coupling coefficients $l_{i j l k}, \lambda_{i j l k}$, and $\rho_{i j l k}$ are given in Appendix A.3. Note, that for $n=3 l, l=1,2,3, \ldots$ there are additional contributions to the amplitude equations due to triad resonances. In the following we focus on planforms where $n$ is no multiple of three. We decompose Eq. (5.20) into the 

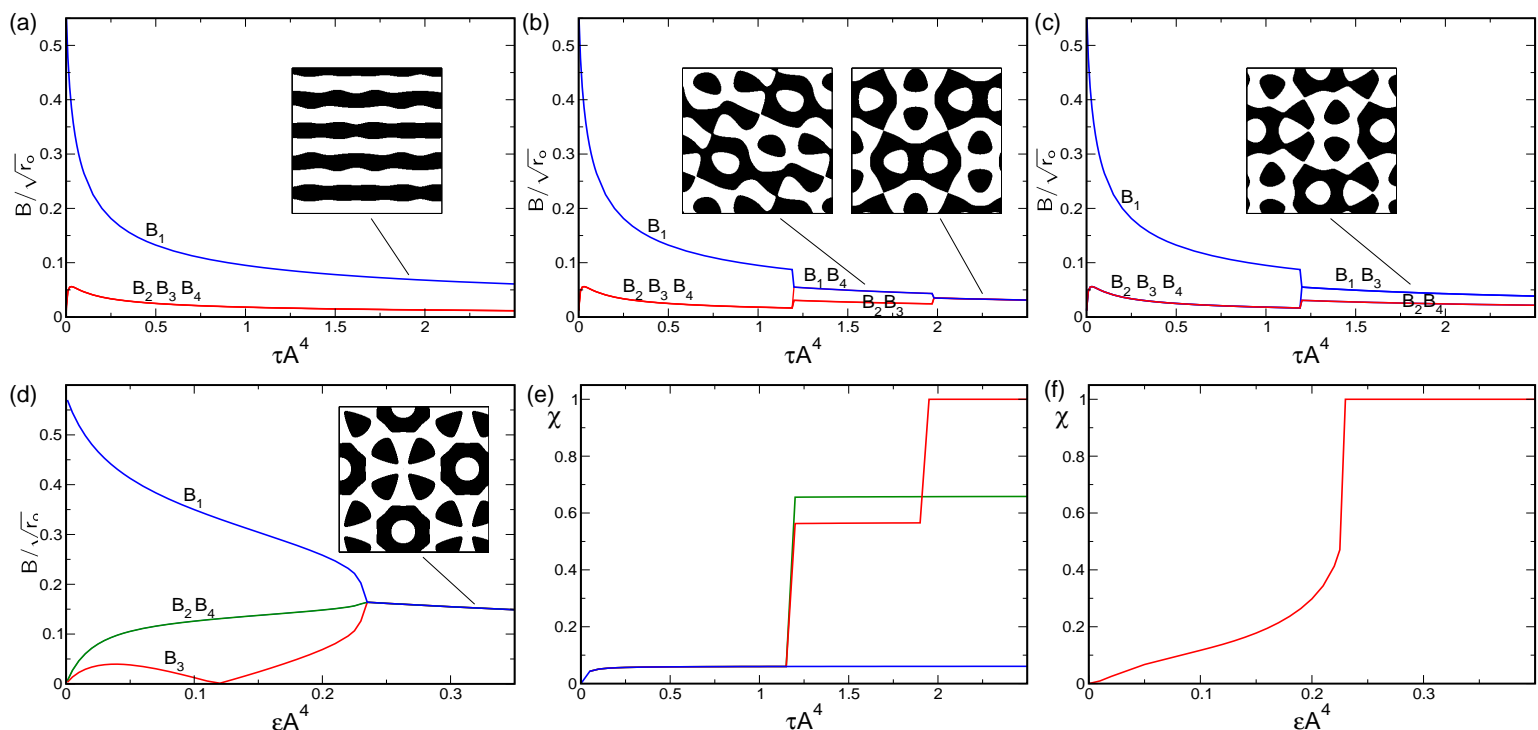

Figure 5.4.: Transition towards uniform solutions. (a)-(c) Stationary solutions of Eq. (5.20) with the coupling energy $U=\tau|z|^{4} o^{4}, n=4$. (d) Stationary solutions with the coupling energy $U=\epsilon|\nabla z \nabla o|^{4}, n=4$. (e,f) Uniformity parameter, see Eq. (5.28).

amplitude

$$
\begin{aligned}
\partial_{t} \mathcal{B}_{i}= & r_{o} \mathcal{B}_{i}-\sum_{j}^{n}\left(\tilde{g}_{i j}+\tilde{h}_{i j}\right) \mathcal{B}_{j}^{2} \mathcal{B}_{i}-\sum_{j, l, k}^{n} l_{i j l k} \mathcal{A}_{i} \mathcal{A}_{j} \mathcal{A}_{l} \mathcal{A}_{k} \mathcal{B}_{l} \mathcal{B}_{k} \mathcal{B}_{j} \cos \Omega_{i j l k} \\
& -\sum_{j, l, k}^{n} \lambda_{i j l k} \mathcal{A}_{j} \mathcal{A}_{l} \mathcal{A}_{k}^{2} \mathcal{B}_{j} \mathcal{B}_{l} \mathcal{B}_{i} \cos \Omega_{j l}-\sum_{j, l, k}^{n} \rho_{i j l k} A_{i} A_{l} A_{k}^{2} B_{j}^{2} B_{l} \cos \Omega_{i l}
\end{aligned}
$$

and phase dynamics

$$
\begin{aligned}
\partial_{t} \psi_{i}= & -\sum_{j, l, k}^{n} l_{i j l k} \frac{\mathcal{A}_{i} \mathcal{A}_{j} \mathcal{A}_{l} \mathcal{A}_{k} \mathcal{B}_{l} \mathcal{B}_{k} \mathcal{B}_{j}}{\mathcal{B}_{i}} \sin \Omega_{i j l k}-\sum_{j, l, k}^{n} \lambda_{i j l k} \mathcal{A}_{j} \mathcal{A}_{l} \mathcal{A}_{k}^{2} \mathcal{B}_{j} \mathcal{B}_{l} \sin \Omega_{j l} \\
& -\sum_{j, l, k}^{n} \rho_{i j l k} \mathcal{A}_{i} \mathcal{A}_{l} \mathcal{A}_{k}^{2} \frac{\mathcal{B}_{j}^{2} \mathcal{B}_{l}}{\mathcal{B}_{i}} \sin \Omega_{i l}
\end{aligned}
$$

with $\Omega_{i j l k}=\left(\phi_{i}+\phi_{j}-\phi_{l}-\phi_{k}\right)-\left(\psi_{i}+\psi_{j}-\psi_{l}-\psi_{k}\right)$ and $\Omega_{i j}=\Omega_{i l j l}$. In the following we identify classes of stationary solutions of the amplitude equations Eq. (5.20) and provide their stability criteria.

\subsection{Uniform solutions}

In this section we identify an analytically tractable class of stationary solutions to the amplitude equations (5.20). We characterize these solutions and study their stability properties. First, we solve Eq. (5.20) numerically. The results in the case of an $n=4$ planform are depicted in Fig. 5.4. We observe that for both types of higher order inter-map coupling energies there is a transition towards a uniform solution $B_{j}=\mathcal{B} e^{\imath \psi_{j}}$. For the gradient-type 
inter-map coupling the uniform solution is the only stable solution above $\epsilon \mathcal{A}^{4} \approx 0.23$. For the product-type inter-map coupling we observe a multistability between the uniform solution, stripe-like solutions and solutions with two dominant modes.

In the following we study uniform solutions for general $n$. An analytically tractable class of solutions to Eq. (5.20) is given by

$$
\begin{aligned}
A_{j} & =\mathcal{A} e^{\imath \phi_{j}}, j=1, \ldots, n \\
A_{j^{-}} & =\mathcal{A}_{-} e^{\imath \phi_{j^{-}}}, \quad \phi_{j-}+\phi_{j}=\phi_{0} \\
B_{j} & =\mathcal{B} e^{\imath \psi_{j}}, \quad \psi_{j}=l_{j}\left(j \frac{2 \pi \Delta}{n}+\phi_{j}\right), \Delta=0, \ldots, n-1 .
\end{aligned}
$$

In the uncoupled case $\phi_{0}$ is an arbitrary phase whereas in the coupled case the phase $\phi_{0}$ depends on the inter-map coupling strength. The phases $\psi_{j}$, however, are independent of the inter-map coupling strength. They depend on the phases $\phi_{j}$ which couples the OP and OD pattern such that they are invariant under a combined translation. The factor $l_{j}$ takes into account the planform dependencies. For a given $n$ there are thus several different uniform solutions. As in the case of pinwheel crystals, Eq. (4.81), we identify a class of solutions which depends on a single parameter $\Delta$. For $n \geq 4$, both the OP and the OD pattern have a spatially irregular layout. As we are interested in the limit $r_{o} \ll r_{z}$ we assume that the modes $A_{j}$ and $A_{j-}$ behave approximately as in the uncoupled case and set $\mathcal{A}_{-}=0$. In this case the amplitude of the OD map is given by

$$
\mathcal{B}_{\Delta}=\sqrt{\frac{r_{o}}{3+6(n-1)+h_{n}+f_{n, \Delta}}} .
$$

The functions $h_{n}$ and $f_{n, \Delta}$ are given by summation over all coupling terms in Eq. (5.20).

$$
h_{n}=\sum_{j}^{n} h_{i j}=\epsilon \mathcal{A}^{4}\left(8 n^{3}-12 n^{2}+\frac{9}{2} n\right)+\tau \mathcal{A}^{4}\left(24 n^{3}-24 n^{2}+6 n\right) \text {, }
$$

and the summation over the coupling coefficients

$f_{n, \Delta}=\mathcal{A}^{4} \sum_{j, l, k}^{n}\left(l_{i j l k} \cos \Omega_{i j l k}+\lambda_{i j l k} \cos \Omega_{j l}+\rho_{i j l k} \cos \Omega_{i l}\right)$ leads to

$f_{n, \Delta}=-(56 \epsilon+96 \tau) n^{2}+(246 \epsilon+240 \tau) n-396 \epsilon-198 \tau+\tilde{f}_{n, \Delta}$ with

$$
\tilde{f}_{n, \Delta}=\left\{\begin{array}{cl}
(55 \epsilon+120 \tau) n^{3}-(220 \epsilon+312 \tau) n^{2}+\left(\frac{1167}{4} \epsilon+246 \tau\right) n & \Delta=0 \\
\frac{17}{2} \epsilon n^{3}-\frac{101}{2} \epsilon n^{2}+\frac{865}{8} \epsilon n & \Delta=1 \\
-\frac{95}{8} \epsilon n & \Delta=\frac{n}{2}-1 \\
\frac{3}{2} n \epsilon & \Delta=2 \\
\frac{27}{4} n \epsilon+6 n \tau & \Delta=n / 2 \\
\frac{5}{2} n \epsilon & \Delta=n-\frac{n+1}{2}
\end{array}\right.
$$

Uniform solutions with $\Delta$ and $(n-\Delta)$ are identical in the sense that they have the same stationary amplitudes $\mathcal{B}_{\Delta}=\mathcal{B}_{n-\Delta}$ and also share the same stability properties. Individual realizations with the phases $\psi_{\Delta}\left(\phi_{j}\right)$ and $\psi_{n-\Delta}\left(\phi_{j}\right)$, however, are not identical. The ECP 
(a)

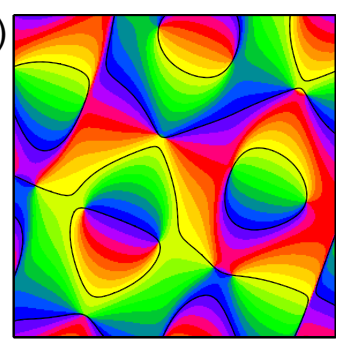

(b)

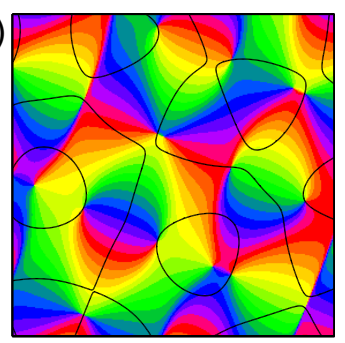

(c)

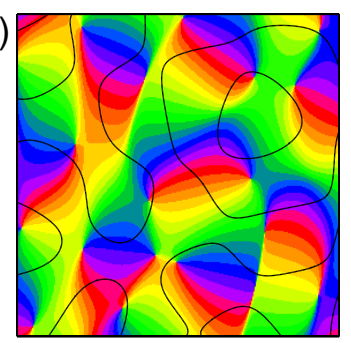

(d)

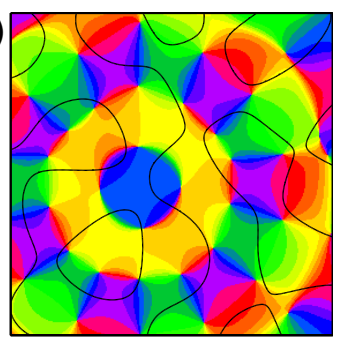

Figure 5.5.: Examples of uniform solutions, Eq. (5.27). OP map with superimposed OD borders. (a) $n=4, \Delta=0$ (LDP), (b) $n=4, \Delta=2$ (LDP), (c) $n=7, \Delta=2$ (LDP), (d) $n=7, \Delta=2(\mathrm{HDP})$.

solutions are degenerate in their phases $\phi_{j}$ thus geometric correlations between OD and OP maps are obtained by averaging over the phases $\phi_{j}$. When averaging over this ensemble the geometric correlations are identical for $\Psi_{\Delta}\left(\phi_{j}\right)$ and $\Psi_{n-\Delta}\left(\phi_{j}\right)$. Examples of uniform solutions are shown in Fig. 5.5. In case of $\Delta=0$ solutions $\phi_{j}=\psi_{j}$ and thus the OD contour lines are identical with the iso-orientation lines, see Fig. 5.5(a). It turns out that the $\Delta=0$ solutions are stable only for $\epsilon<0(\tau<0)$ for which the corresponding coupling energy is negative and potentially destabilizes the dynamics. For solutions with $\Delta>0$, see Fig. 5.5(c-d), the phase relations adjust the contour lines such that the iso-orientation lines tend to intersect the OD border perpendicularly. A quantitative analysis of intersection angles and the distribution of pinwheel positions will be provided in Section 5.8. In the limit $r_{o} \ll r_{z}$ the attractors of $A_{j}$ and $A_{j^{-}}$are those of the uncoupled case i.e.

$$
\begin{aligned}
& A_{j}=\mathcal{A} e^{\imath \phi_{j}}, A_{j^{-}}=0 \\
& B_{j}=\mathcal{B} e^{\imath \psi_{j}}, \psi_{j}=l_{j}\left(j \frac{2 \pi \Delta}{n}+\phi_{j}\right), j=0, \ldots, n-1
\end{aligned}
$$

with $\mathcal{A}=\sqrt{r_{z} / \sum_{j} g_{i j}}$. In the following we study the stability properties of Eq. (5.27) by linear stability analysis. The stability properties of solution Eq. (5.23) are determined numerically.

\subsubsection{Measures of uniformity}

To discriminate uniform solutions from non-uniform ones we introduce the uniformity parameter

$$
\chi\left(B_{1}, \ldots, B_{n}\right)=\left(\frac{\sum_{j=0}^{n-1}\left|B_{j}\right|^{2}}{\max _{j}\left|B_{j}\right|^{2}}-\left|\frac{\sum_{j=0}^{n-1}\left|B_{j}\right|^{2} e^{2 \imath \alpha_{i}}}{\sum_{j=0}^{n-1}\left|B_{j}\right|^{2}}\right|\right) / n .
$$

This parameter ranges from $\chi=0$ for stripe solutions to $\chi=1$ for a uniform solution, see Fig. 5.4 (e,f). When studying amplitude equations $\chi$ is a suitable measure to identify uniform solutions. In case of numerical simulations discrete Fourier modes are not always present and the spectrum can smeared out for instance due to the presence of distinct domains. As a second measure which does not rely on counting distinct Fourier modes we therefore use the bandedness $b$ of the OD pattern which was introduced in [79]. Based on a wavelet 
transformation the bandedness uses the orientation dependence of the wavelet coefficients, see Appendix A.4. The bandedness therefore measures the anisotropy of the pattern. For a stripe pattern bandedness is high while for an isotropic pattern such as uniform solutions or random fields bandedness is low.

\subsubsection{Stability properties}

We study the stability properties of the stationary solutions Eq. (5.27) under perturbations of phases and of the amplitudes

$$
\mathcal{B}_{j} e^{\imath \psi_{j}} \Rightarrow\left(\mathcal{B}+b_{j}\right) e^{\imath\left(\psi_{j}+\varphi_{j}\right)}
$$

We set $\mathcal{A}_{j}=\mathcal{A}$. For small phase perturbations we can expand the sine and cosine in Eq. (5.21), Eq. (5.22) as $\sin \left[\Omega_{i j l k}-\varphi_{i}-\varphi_{j}+\varphi_{l}+\varphi_{k}\right] \approx \sin \Omega_{i j l k}+\cos \Omega_{i j l k}\left(-\varphi_{i}-\varphi_{j}+\varphi_{l}+\varphi_{k}\right)$, $\cos \left[\Omega_{i j l k}-\varphi_{i}-\varphi_{j}+\varphi_{l}+\varphi_{k}\right] \approx \cos \Omega_{i j l k}-\sin \Omega_{i j l k}\left(-\varphi_{i}-\varphi_{j}+\varphi_{l}+\varphi_{k}\right)$. Using the stationarity condition we thus obtain

$$
\begin{aligned}
\partial_{t} b_{i}= & r_{o} b_{i}-\sum_{j}\left(\widetilde{g}_{i j}+\tilde{h}_{i j}\right)\left(2 \mathcal{B}^{2} b_{j}+\mathcal{B}^{2} b_{i}\right) \\
& -\sum_{j l k} l_{i j l k} \mathcal{B}^{2}\left(b_{j}+b_{l}+b_{k}\right) \cos \left(\Omega_{i j l k}\right) \\
& -\sum_{j l k} l_{i j l k} \mathcal{B}^{3} \sin \Omega_{i j l k}\left(\varphi_{i}+\varphi_{j}-\varphi_{l}-\varphi_{k}\right),
\end{aligned}
$$

and

$$
\begin{aligned}
\partial_{t} \varphi_{i}= & \sum_{j l k} l_{i j l k} \mathcal{B}^{2} \cos \Omega_{i j l k}\left(\varphi_{i}+\varphi_{j}-\varphi_{l}-\varphi_{k}\right) \\
& -\sum_{j l k} l_{i j l k} \mathcal{B}\left(b_{j}+b_{l}+b_{k}\right) \sin \Omega_{i j l k} .
\end{aligned}
$$

Not shown are contributions resulting from $\lambda$ and $\rho$ terms. In general phase and amplitude perturbations do not decouple. We therefore combine both linear equations as

$$
\partial_{t} v_{i}=\sum_{j}^{2 n} M_{i j} v_{j}, \quad i=1, \ldots, 2 n, \quad M=\left(\begin{array}{cc}
M_{1} & M_{2} \\
M_{3} & M_{4}
\end{array}\right),
$$

with $v=\left\{b_{1}, \ldots, b_{n}, \varphi_{1}, \ldots \varphi_{n}\right\}$. If amplitude and phase perturbations decouple the eigenvalues of the matrix $M$ can be derived analytically since $M_{1}$ and $M_{4}$ are circulant matrices $M_{i j}=m_{(j-i)} \bmod n$ and thus their eigenvalues are given by $\lambda_{1, l}=\sum_{j} M_{1,1 j} \cos (2 \pi(j-1)(l-1) / n), l=1, \ldots n$. A decoupling of amplitude and phase perturbations occurs only in the cases $\Delta=n / 2$ or $\Delta=0$. In the remaining cases the eigenvalues are calculated numerically. In the following we discuss the stability properties of all uniform solutions for a given number of active modes $n$. The exceptional case of OP 

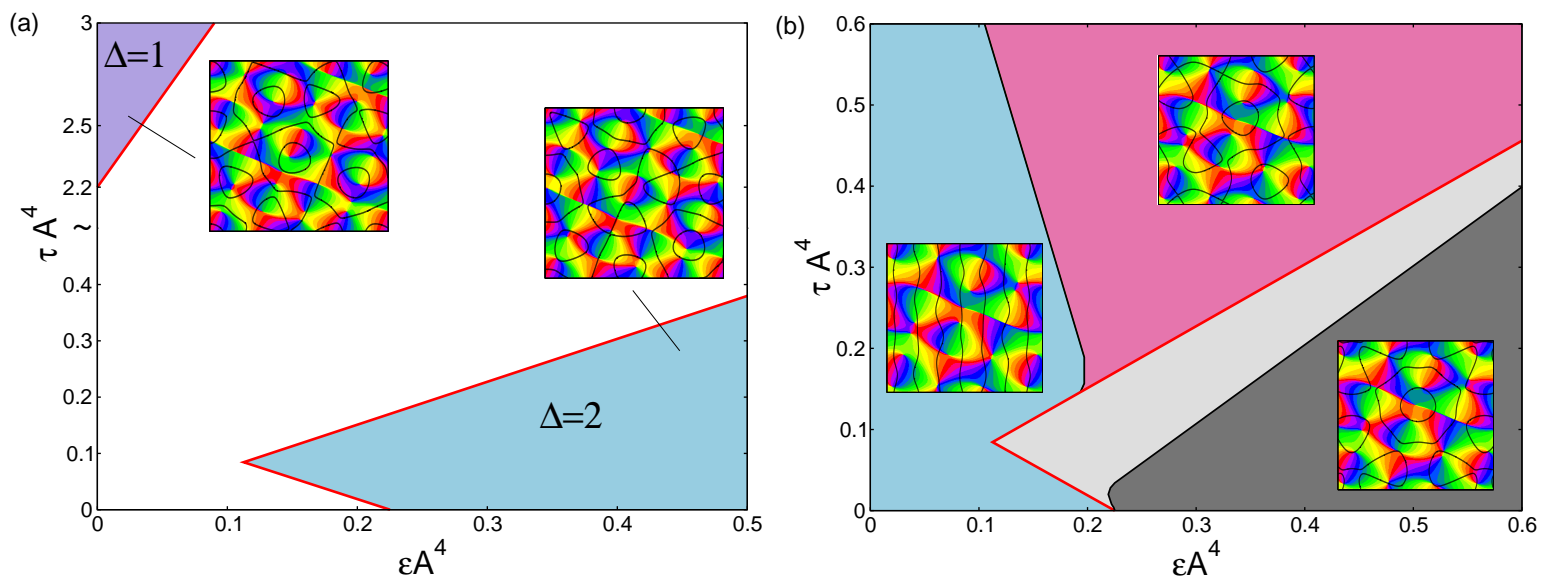

Figure 5.6.: Phase diagram for $\mathbf{n}=4$. (a) Stability range of the $n=4, \Delta=1$ and $\Delta=2$ uniform solutions. Insets: fixed OP map, superimposed OD borders according to Eq. (5.27). (b) Phase diagram for $n=4, r_{o} \ll r_{z}$. The red line: stability border of the $\Delta=2$ solution. The light blue region indicates the stability of stripe-like solutions $\mathcal{B}=\left(\mathcal{B}_{2}, \mathcal{B}_{1}, \mathcal{B}_{2}, \mathcal{B}_{3}\right)$, $\mathcal{B}_{1} \gg \mathcal{B}_{2}, \mathcal{B}_{3}$. The light red region indicates the stability region of the solution $\mathcal{B}=\left(\mathcal{B}_{1}, \mathcal{B}_{2}, \mathcal{B}_{1}, \mathcal{B}_{2}\right)$. The dark gray region indicates the stability of the $\Delta=2$ uniform solution. The light gray region is a region of bistability between the uniform solution and solutions with two dominant modes.

stripes $(n=1)$ leads to OD stripes with independent phase configurations. In case of the gradient-type coupling energy both stripes are perpendicular as already observed in the previous chapter. In case of the product-type coupling energy the OD stripes run in an arbitrary direction as orientation shift symmetry prevents a preference for a parallel configuration. In case of $n=2$ the $\Delta=1$ solution is stable for $\epsilon \mathcal{A}^{4}+\tau \mathcal{A}^{4}>0.5$. For $n=3,6,9, \ldots$ there are additional contributions to the amplitude equations due to triad resonances. In this case the described uniform solutions do not exist. Instead we find stable non-uniform solutions which can become very close to uniformity $(\chi>0.9)$. The more interesting cases are given for $n \geq 4$ as the OP layout in this case is spatially irregular. Figure 5.6 shows the phase diagram for the $n=4$ solutions. The phase diagram is drawn in terms of the effective coupling strength on the OD map $\epsilon \mathcal{A}^{4}$ and $\tau \mathcal{A}^{4}$. For $\epsilon>0$ and $n=4$ a stable uniform solution is the $\Delta=2$ solution. Here the amplitude and phase perturbations do decouple. We therefore get two independent stability borders. The one for the amplitude perturbation is given by $\mathcal{A}^{4} \epsilon=\frac{3-18 \mathcal{A}^{4} \tau}{13}$ while the border for the phase perturbations is given by $\epsilon=\frac{4}{3} \tau$. The stability borders are indicated by red lines. The $\Delta=1$ uniform solution is not stable for $\tau=0$. However, it becomes stable above $\tau \mathcal{A}^{4} \approx 2.2(\epsilon=0)$. In case of $n=4$ we identified two types of non-uniform solutions namely stripe-like solutions $\mathcal{B}=\left(\mathcal{B}_{2}, \mathcal{B}_{1}, \mathcal{B}_{2}, \mathcal{B}_{3}\right), \mathcal{B}_{1} \gg \mathcal{B}_{2}, \mathcal{B}_{3}$ and solutions with two dominant amplitudes $\mathcal{B}=\left(\mathcal{B}_{1}, \mathcal{B}_{2}, \mathcal{B}_{1}, \mathcal{B}_{2}\right)$ with $\chi=\frac{1}{2}+\frac{1}{2} \frac{\mathcal{B}_{2}^{2}}{\mathcal{B}_{1}^{2}}$. In case of the gradient-type inter-map coupling we find stable uniform solutions also for $n=5(\Delta=2)$ and $n=7(\Delta=2,3)$.

Figure 5.7 shows the phase diagram for the $n=8$ solutions. Here, there are two stable uniform solutions, the $\Delta=2$ and $\Delta=3$ solution. The $\Delta=3$ solution has a larger stability range. The stability borders for the non-uniform solutions are obtained by solving 

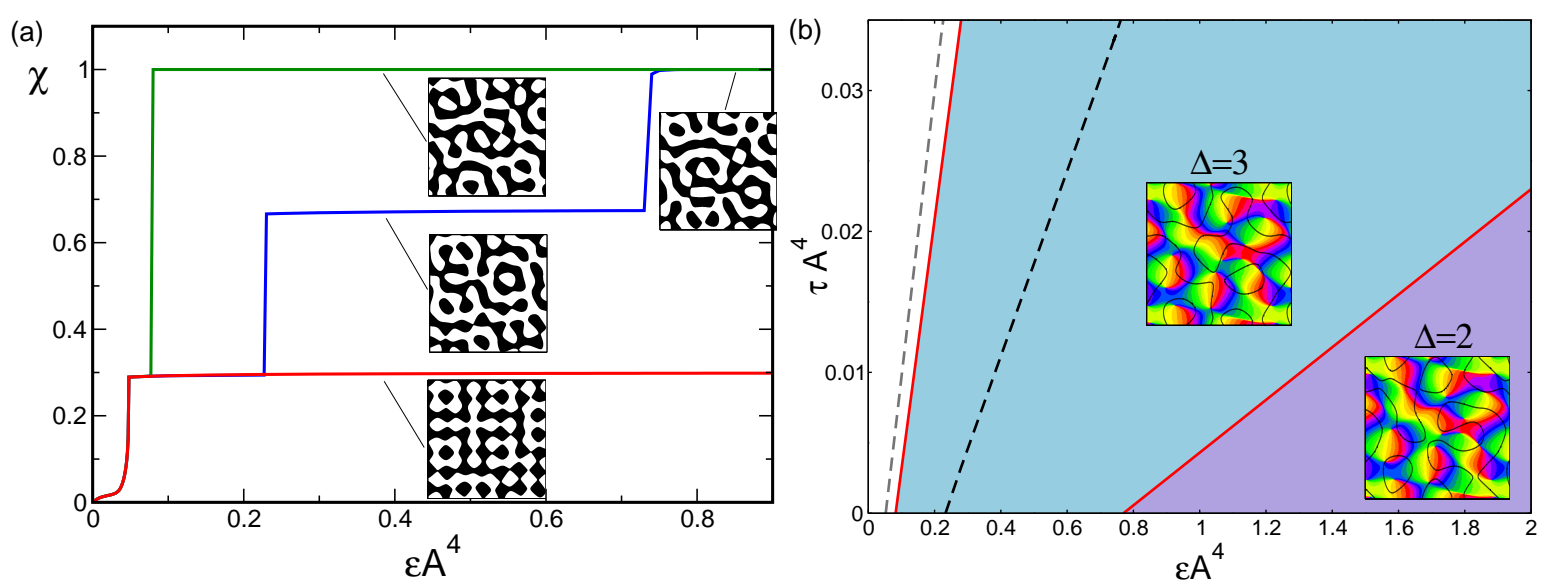

Figure 5.7.: Phase diagram for $\mathbf{n}=\mathbf{8}$. (a) Uniformity parameter Eq. (5.28), $\tau=0$, red: solution with two dominant modes, blue: solution with four dominant modes, green: uniform solution. (b) Phase diagram for $n=8, r_{o} \ll r_{z}$. The red lines indicate the stability line of the $\Delta=2$ and $\Delta=3$ uniform solutions. Gray dashed line: stability border of stripe-like solutions. Black dahsed line: stability border of solution with four dominant modes.

the amplitude equations (5.20) numerically. In case of $n=8$ we identified three classes of non-uniform solutions: Stripe-like solutions with one dominant mode, solutions with two dominant modes $\mathcal{B}=\left(\mathcal{B}_{1}, \mathcal{B}_{2}, \mathcal{B}_{1}, \mathcal{B}_{3}, \mathcal{B}_{1}, \mathcal{B}_{2}, \mathcal{B}_{1}, \mathcal{B}_{3}\right)$ with $\mathcal{B}_{3} \gg \mathcal{B}_{1}, \mathcal{B}_{2}\left(\chi=\frac{1}{4}+\frac{1}{4} \frac{\mathcal{B}_{2}^{2}}{\mathcal{B}_{3}^{2}}+\frac{1}{2} \frac{\mathcal{B}_{1}^{2}}{\mathcal{B}_{3}^{2}} \approx 0.3\right)$, and solutions with four dominant modes $\mathcal{B}=\left(\mathcal{B}_{1}, \mathcal{B}_{2}, \mathcal{B}_{1}, \mathcal{B}_{2}, \mathcal{B}_{1}, \mathcal{B}_{2}, \mathcal{B}_{1}, \mathcal{B}_{2}\right)$ with $\mathcal{B}_{1}>\mathcal{B}_{2}$ $\left(\chi=\frac{1}{2}+\frac{1}{2} \frac{B_{2}^{2}}{B_{1}^{2}} \approx 0.66\right)$. Stripe-like solutions which are stable for small $\epsilon$ loose their stability above a critical inter-map coupling strength (gray dashed line). Above this line solutions with two dominant modes are stable. Between the black dashed line and the red line solutions with four dominant modes are stable. In contrast to $n=4$, in the case of $n=8$ uniform solutions are not stable for $\epsilon=0, \tau \geq 0$. For large inter-map coupling strength there is a multistability of the $\Delta=2$ and $\Delta=3$ uniform solutions as well as of the non-uniform solution with two dominant modes. We therefore check which of these solutions corresponds to the ground state of energy. To this end we calculate the potential

$$
\begin{aligned}
V[A, B]= & -r_{o} \sum_{i}\left|B_{i}\right|^{2}-r_{z} \sum_{i}\left|A_{i}\right|^{2}+\frac{1}{2} \sum_{i, j} \widetilde{g}_{i j}\left|B_{i}\right|^{2}\left|B_{j}\right|^{2}+\frac{1}{2} \sum_{i, j} g_{i j}\left|A_{i}\right|^{2}\left|A_{j}\right|^{2} \\
& +\frac{1}{2} \sum_{i, j, l, k} h_{i j l k}\left|A_{j}\right|^{2}\left|A_{l}\right|^{2}\left|B_{k}\right|^{2}\left|B_{i}\right|^{2}+\frac{1}{2} \sum_{i, j, l, k} l_{i j l k} A_{i} A_{j} \bar{A}_{k} \bar{A}_{l} \bar{B}_{i} \bar{B}_{j} B_{k} B_{l} \\
& +\frac{1}{2} \sum_{i, j, l, k} \rho_{i j l k} A_{i} \bar{A}_{l}\left|A_{k}\right|^{2}\left|B_{j}\right|^{2} B_{l} \bar{B}_{i}+\frac{1}{2} \sum_{i, j, l, k} \lambda_{i j l k} A_{j} \bar{A}_{l}\left|A_{k}\right|^{2} \bar{B}_{j} B_{l}\left|B_{i}\right|^{2}
\end{aligned}
$$

Since $l_{i j l k}=l_{l k i j}, \lambda_{i j l k}=\lambda_{i l j k}$ and $\rho_{i j l k}=\rho_{l j i k}$ the potential is real. It turns out that the $\Delta=3$ uniform solution is for all $\epsilon>0$ energetically preferred compared to the $\Delta=2$ solution and that the energy difference decreases with increasing coupling strength $\epsilon$. The non-uniform solution with two dominant modes, however, is energetically preferred compared to the uniform solutions.

To summarize, we studied the stability properties of uniform solutions for a different number 
of active modes $n$. It turns out that within the class of uniform solutions different inter-map coupling energies select different stable solutions. We discussed in detail the cases $n=4$, because it is the simplest ECP with a quasiperiodic pattern, and $n=8$, because the OP layout already resembles that of realistic OP maps. For $n \geq 7$ there is a multistability between different uniform solutions. In addition for $n \geq 7$ there is a multistability between uniform solutions and non-uniform solutions. In case of the product-type inter-map coupling we found stable uniform solutions only for $n=2$ and $n=4$ whereas for higher $n$ we found stable non-uniform solutions which are in general more stripe-like than those found in case of the gradient-type inter-map coupling.

\subsection{Including the backreaction}

For $r_{o} \ll r_{z}$ and higher order inter-map coupling terms we could neglect the influence of the backreaction onto the OP map. How robust are the previous results against the influence of a finite backreaction? In this section we show that uniform solutions become stable even for a considerable amount of backreaction, although their stability borders are shifted towards higher inter-map coupling strength.

For a finite ratio $r_{o} / r_{z}$ we have to consider the complete coupled dynamics of the modes $B_{j}, A_{j}$ and $A_{j^{-}}$. If we include this backreaction we see that solutions with $A_{j^{-}}=0$ are unstable but the amplitudes of the opposite wavevectors are still small for small enough $r_{o} / r_{z}$. We solve the coupled system of modes Eq. (5.16) numerically. The course of the amplitudes in case of a $n=4 \mathrm{LDP}$ is shown in Fig. 5.8. Note, the inter-map coupling is rescaled as $\epsilon \mathcal{A}^{4}$ where $\mathcal{A}$ denotes the uncoupled amplitude of the OP map. For $r_{o} / r_{z}=0.1$ the backreaction to the OP map is still small and thus the amplitudes $A_{j}$ and $A_{j^{-}}$deviate only slightly from those of the uncoupled case, see Fig. 5.8 (a). At $\epsilon \mathcal{A}^{4} \approx 0.3$ there is a transition towards the $\Delta=2$ uniform solution which is at a slightly higher coupling than in the case without backreaction where $\epsilon \mathcal{A}^{4}=3 / 13 \approx 0.23$. Remarkably, the ratio $r_{o} / r_{z}$ can be increased even to $r_{o} / r_{z}=1$, see Fig. 5.8(b). Here, for small coupling $\epsilon \mathcal{A}^{4}$ the backreaction makes the $n=4$ ECP solution unstable and the OP map consists of stripes. Increasing inter-map coupling at some point, $\epsilon \mathcal{A}^{4} \approx 18$, again leads to a transition towards the uniform solution. Without backreaction the $n=4$ uniform solution is the only stable solution above a critical inter-map coupling strength. When the backreaction is taken into account we observe a bistability between uniform and stripe solutions, even for large inter-map coupling. Moreover, the region of bistability increases with increasing backreaction. Increasing the backreaction even more will at some point make the uniform solution unstable. Figure 5.8(c) shows the gradual change in the stability borders of an $n=4, \Delta=2$ planform with increasing backreaction. Compared to the situation without backreaction (black line) the backreaction shifts the critical map coupling at which the $\Delta=2$ uniform solution becomes stable towards higher values (red line). We compared these results with stability borders obtained by solving the full field dynamics numerically. Here we expect, apart from the influence of the backreaction, two main reasons for deviations from the amplitude equations. First, higher order corrections to the amplitude 

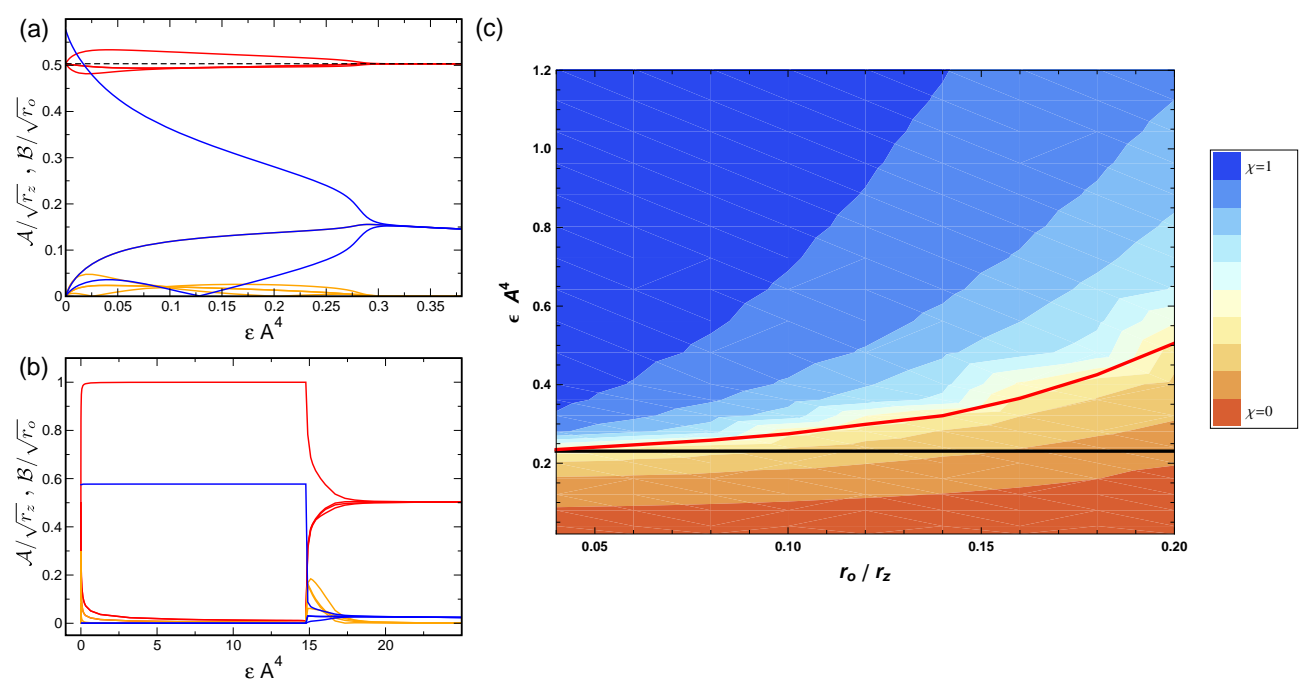

Figure 5.8.: Backreaction on the OP map (a,b) Stationary amplitudes (a) $r_{o}=0.01, r_{z}=0.1$, (b) $r_{o}=0.1, r_{z}=0.1$, dashed lines: $\mathcal{A}(\epsilon=0, g=0.98, \sigma=0.7 \Lambda)$. Red: $\mathcal{A}_{j}$, orange: $\mathcal{A}_{j^{-}}$, blue: $\mathcal{B}_{j}$. (c) Influence of the backreaction on the stability border of the $n=4, \Delta=2$, LDP uniform solution. Black line: critical inter-map coupling when changing the ratio $r_{o} / r_{z}$, keeping $r_{z}=0.25$ fixed, no backreaction. Red line: with backreaction. The results are compared with solutions of the full field dynamics Eq. (5.1). Color codes for the uniformity parameter $\chi$, see Eq. (5.28). Parameters:

$g=0.98, \sigma=0.7 \Lambda, T_{f}=5 \cdot 10^{4}, \Gamma=36, \operatorname{mesh}=256 \times 256$.

equations which have to be considered for larger values of $r_{z}$ and $r_{o}$, and second deviations due to spatial discretization, see Appendix A.1. We observe a similar increase in the stability border when increasing the backreaction. However, the transition towards uniform solution becomes much smoother with larger backreaction. The borders are, as expected, slightly above the borders obtained from amplitude equation but are still comparable. For $r_{o} / r_{z}$ small the transition towards the uniform solution is in good agreement with the predictions from amplitude equations. For larger $r_{o} / r_{z}$ the transition region increases. Starting from uniform solutions as initial conditions we integrated this solutions until the final time $T_{f}=5 \cdot 10^{4} / r_{z}$. In case of the $n=4$ uniform solutions the final pattern experiences only minor changes and the uniformity parameter for the final state is $\chi \approx 0.97$. To summarize, although the limit $r_{o} \ll r_{z}$ simplifies the analysis of the amplitude equations a transition towards spatially irregular OD solutions is observed also for $r_{o} \approx r_{z}$.

\subsection{Geometric relationships}

To what extent do the different inter-map coupling energies impact on the geometric relationships between OD and OP maps? To answer this question we study these geometric relationships for different uniform solutions as well as for solutions obtained from solving the full field dynamics numerically. Experiments show that iso-orientation lines tend to intersect 
(a)
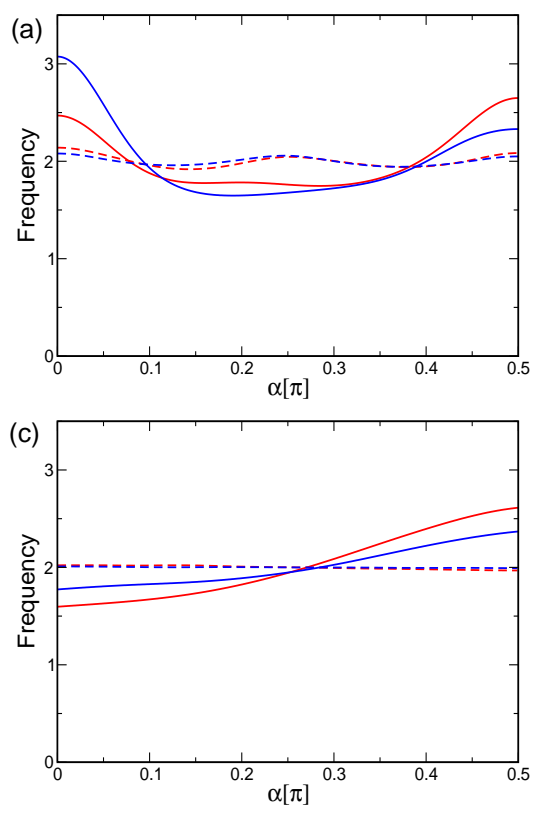

(b)
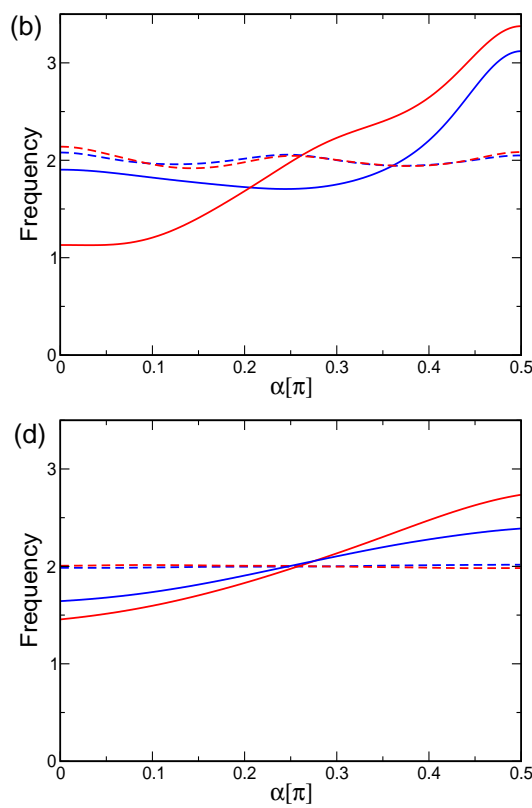

Figure 5.9.: Statistics of intersection angles for uniform solutions, Eq. (5.23). (a) $n=4, \Delta=1$, (b) $n=4, \Delta=2$, (c) $n=8, \Delta=2$, (d) $n=8, \Delta=3$. Red lines: LDP, blue lines: HDP, dashed lines: Intersection angles with random phases $\psi_{j}$.

the borders of OD at right angle. We therefore study the distribution of intersection angles

$$
\alpha(\mathbf{x})=\cos ^{-1}\left(\frac{\nabla o(\mathbf{x}) \cdot \nabla \vartheta(\mathbf{x})}{|\nabla o(\mathbf{x})||\nabla \vartheta(\mathbf{x})|}\right)
$$

where $\mathbf{x}$ denotes the position of the OD zero-contour lines. A continuous expression for the OP gradient is given by $\nabla \vartheta=\operatorname{Im} \nabla z / z$. We emphasize those parts of the maps from which the most significant information about the intersection angles can be obtained [73,75]. These are the regions where the OP gradient is high and thus every intersection angle receives a statistical weight according to $|\nabla \vartheta|$. Examples of intersection angle distributions for uniform solutions are shown in Fig. 5.9, In case of the uniform solution we synthesize $N$ different realizations with random phases $\phi_{j}$. The phases $\psi_{j}$ are calculated according to the formula Eq. (5.23) (solid lines) and for comparison chosen random (dashed lines). The intersection histogram is an average over these realizations. The number of realizations $N$ is chosen such that $S D / \sqrt{N}<0.1$, where $S D$ is the maximal standard deviation of intersection angles in the region $0 \geq \alpha \geq 0.2 \pi$. The afforded precision required between about 100 and 1000 realizations $(\Gamma=24$, mesh $=1024 \times 1024)$.

In case of the $n=4, \Delta=1$ solution, see Fig. 5.9 (a), which is stable in case of the product-type coupling energy we obtain a bimodal distribution of intersection angles with peaks at $\alpha=0$ and $\alpha=\pi / 2$. In contrast, stationary solutions of the gradient-type coupling energy all show a tendency towards perpendicular intersection angles. This bias is in general more pronounced for LDP solutions (red lines) than for HDP solutions (blue lines). A reason for this might be the higher anisotropy in the layout of LDP solutions. Also for small $n$, see Fig. 5.9(b), this 


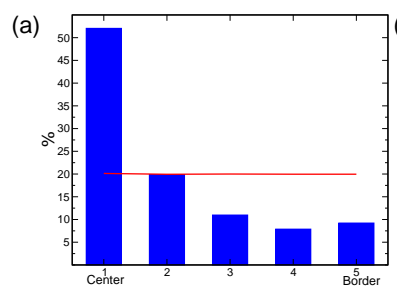

(e)

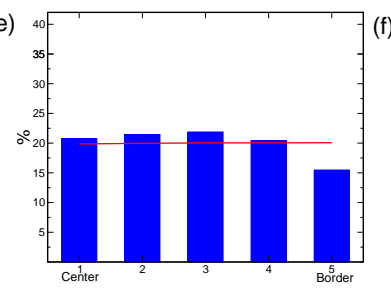

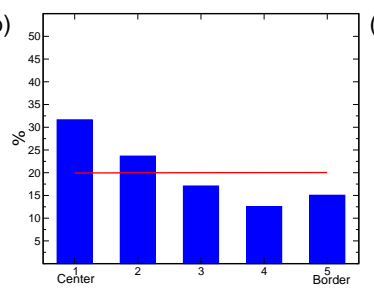

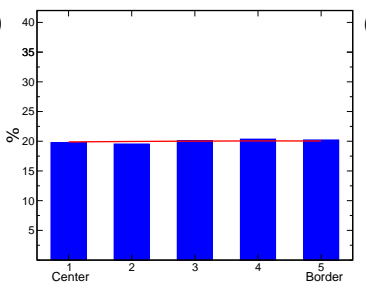

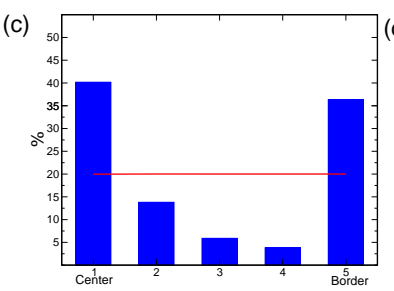

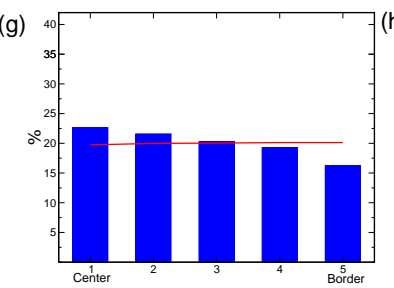

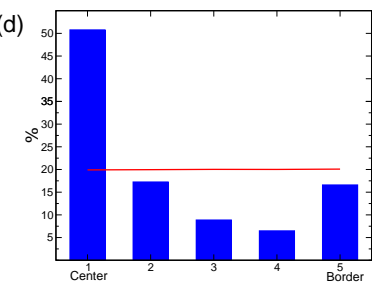

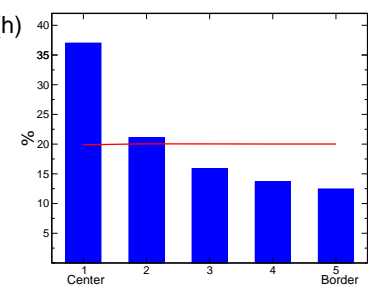

Figure 5.10.: Distribution of pinwheel positions for uniform solutions, Eq. (5.23). (a) $n=4, \Delta=1 \mathrm{LDP}$, (b) $n=4, \Delta=1 \mathrm{HDP}$, (c) $n=4, \Delta=2 \mathrm{LDP},(\mathrm{d}) n=4, \Delta=2 \mathrm{HDP}$, (e) $n=8, \Delta=2 \mathrm{LDP}$, (f) $n=8, \Delta=2 \mathrm{HDP}$, (g) $n=8, \Delta=3 \mathrm{LDP}$, (h) $n=8, \Delta=3$ HDP. Red line: Distribution with random phases $\psi_{j}$.

bias is more pronounced than for larger $n$, see Fig. $5.9(\mathrm{c}, \mathrm{d})$. Note, the employed statistical weight $|\nabla \vartheta|$ has only a small effect on the distribution of intersection angles which becomes negligible for large $n$.

A second experimental observation is the tendency for pinwheels to be located in the center regions of OD columns (extrema of OD). To quantify this property we divide the OD maps into five regions. Region 1 corresponds to the 0-10 and 90-100 percentile (peak of the ipsilateral and contralateral eye domains). Region 5 corresponds to the 40-50 and 50-60 percentile (border regions of the OD map) and so on. In case of the uniform solutions we synthesize $N$ different realizations with random phases $\phi_{j}$. The phases $\psi_{j}$ are again calculated according to the formula Eq. (5.23) and for comparison chosen random (red line). The histogram of pinwheel positions is an average over these realizations. The number of realizations $N$ is chosen such that $S D / \sqrt{N}<0.5$ where SD is the maximal standard deviation of the five regions. The afforded precision required between about 300 and 2200 realizations or between 150000 and 630000 counted pinwheels, $(\Gamma=22$, mesh $=1024 \times 1024)$. The distribution of pinwheel positions for some uniform solutions are shown in Fig. 5.10. The red lines indicate the values obtained if the pinwheel centers were distributed randomly. We observe that all stable $n=4$ uniform solution show the tendency for pinwheels to be located at OD extrema. This tendency is most pronounced in case of the $n=4, \Delta=1$ uniform solution which is stable in case of the product-type coupling energy, see Fig. $5.10(a, b)$ We expect that the density of pinwheels has an influence on the distribution of pinwheel positions. To check this we calculated the pinwheel positions for planforms with different pinwheel densities (LDP,HDP). In case of $n=4, \Delta=2$ the pinwheels are preferentially located at the extrema and this preference is higher for the HDP. For $n=8$ the situation is similar although there are planforms where the pinwheels positions are not preferentially at the extrema, see Fig. 5.10(f).

In case of the gradient-type inter-map coupling we systematically study the geometric rela- 

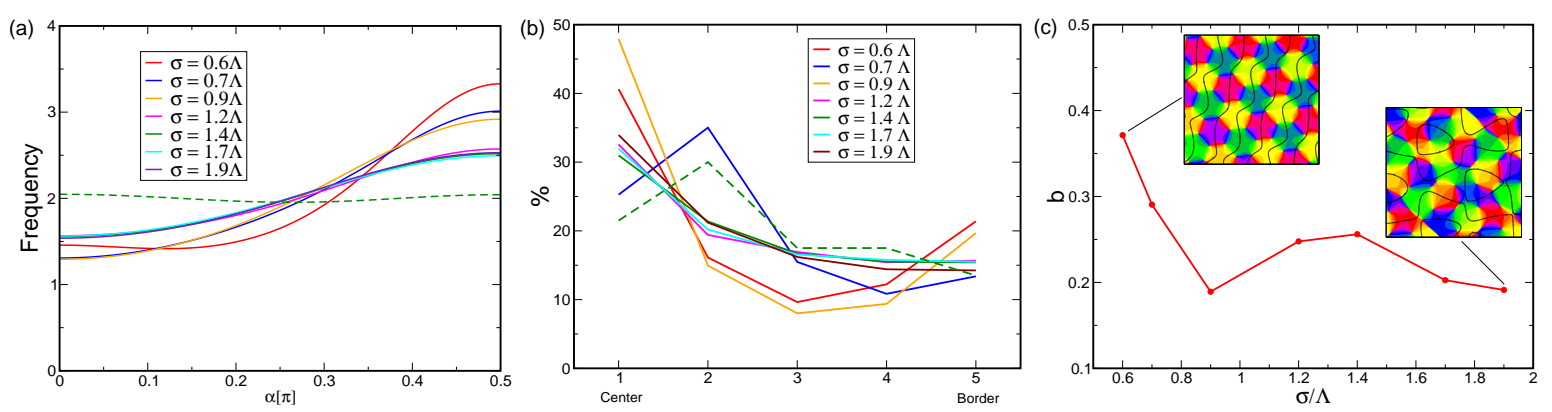

Figure 5.11.: Geometric relationships from final states of the full field dynamics. (a) Intersection angles, solid lines: $\epsilon=2000, \tau=0$, dashed line: $\epsilon=0, \tau=2000$. (b) Distribution of pinwheel positions (c) Mean bandedness of the OD patterns. Parameters: $g=0.98, r_{z}=0.25, r_{o}=0.02, T_{f}=10^{5}, \Gamma=22,128 \times 128$ mesh.

tionships for final states of the full field dynamics, see Fig. 5.11. We vary the length of the long-range interactions between $\sigma=0.6 \Lambda$ and $\sigma=1.9 \Lambda$ which corresponds to planforms with between $n=2$ and $n=9$ active modes. For each value $\sigma$ we averaged over $n=50$ simulations using band-pass filtered Gaussian random fields as initial conditions for both maps. We observe that in all cases there is a bias towards orthogonal intersection angles, see Fig. 5.11(a). This bias is less pronounced for larger $\sigma$ values. This observation is in line with the results from uniform solutions where with increasing $n$ the bias is less pronounced. Moreover, in all cases there is a bias for pinwheels to be located near the center of OD, see Fig. 5.11(b). Except for $\sigma=0.7 \Lambda$ the distributions of pinwheel positions show a peak at the center region of OD. In all cases the bandedness is rather low, see Fig. 5.11(c), and the corresponding OD layout is patchy. We compare these results with simulations using the product-type inter-map coupling, dashed lines in Fig. $\mathbf{5 . 1 1}(\mathrm{a}, \mathrm{b})$. Here, the distribution of intersection angles is almost flat. However, more pinwheels are located in the center than in the border region.

\subsection{Numerical analysis of the coupled dynamics}

In this section we numerically analyze the coupled field dynamics. As in numerical simulations there is a finite backreaction on the OP map we first study how the dynamics of the OP map is influenced by inter-map coupling. We show that pinwheel annihilation is accelerated in a regime where pinwheels are intrinsically unstable. We further show that even when considering a finite backreaction the properties of the uncoupled OP solutions are robust against inter-map coupling. Next, we study the time evolution of the OD layout and demonstrate that inter-map coupling indeed leads to patchy OD maps with a low bandedness. Finally, we study a detuning of the OP and OD typical wavelength. We show that this detuning has an impact on the OD layout and leads to a potential explanation for the species differences in the OD layout. 


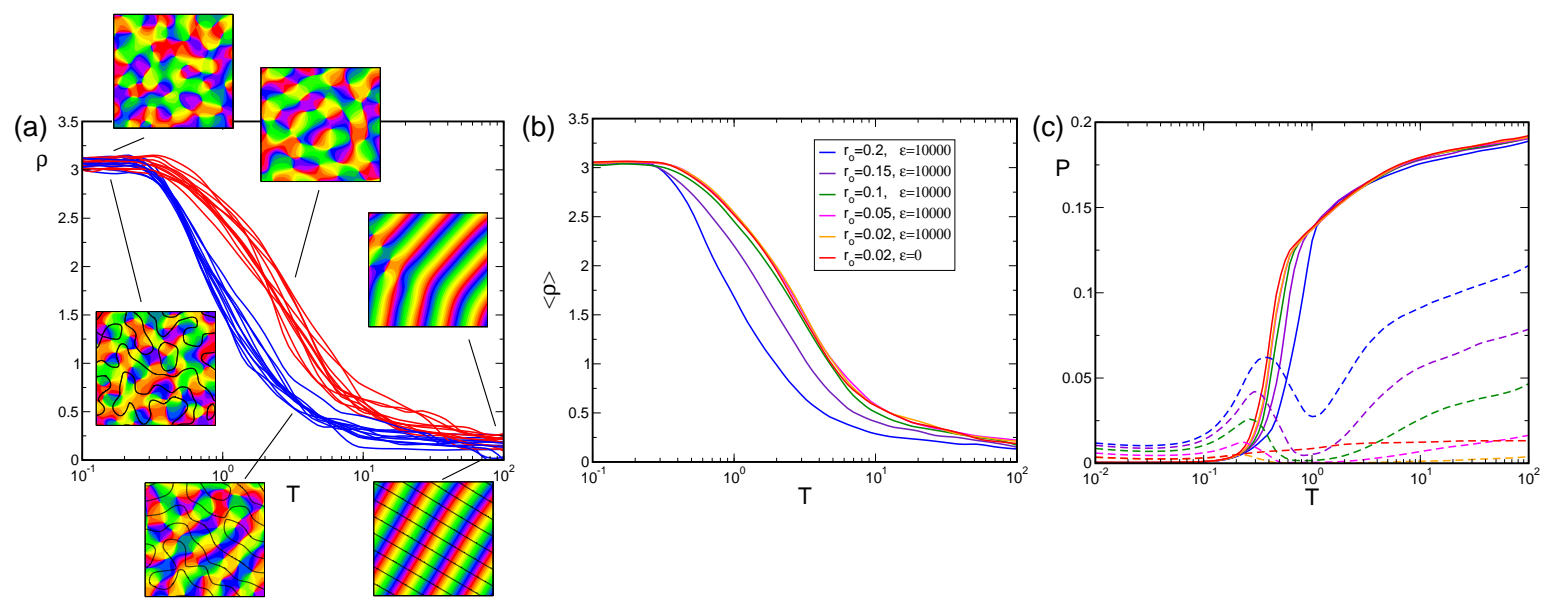

Figure 5.12.: Pinwheel annihilation without long-range interactions $(g=2)$. (a)

Pinwheel density with $(\epsilon=10000$, blue) and without $(\epsilon=0$, red) inter-map coupling for ten initial conditions. (b) Pinwheel density for different bifurcation parameters $r_{o}$. Each line represents an average over the ten simulations. (c) Power of OP maps (solid lines) and OD maps (dashed lines). Each lines represents the average over the ten simulations. Parameters in all simulations: $r_{z}=0.2, g=2, \Gamma=22,128 \times 128$ mesh.

\subsubsection{Pinwheel kinetics}

Here, we test the influence of inter-map coupling on various statistics of pinwheels. Tracking of pinwheels is detailed in Section 3.4. In a regime where pinwheels are intrinsically unstable the influence of the OD map can impede pinwheel annihilation when the OD map is spatially irregular [18,99]. As discussed in the last chapter OD segregation with a patchy OD layout can even stabilize pinwheel rich solutions. Here, we study how regular OD stripes influence the pinwheel annihilation process. In the case of $g=2$ the attractor for the OP map are stripes and a pinwheel rich pattern decays since pinwheels are annihilated. Figure 5.12 shows the time evolution of the pinwheel density in this situation. For each value of the bifurcation parameter $r_{o}$ we started with an identical set of ten random initial conditions. Figure 5.12(b) shows the average pinwheel density of these simulations. We observe that compared to the uncoupled case the inter-map coupling accelerates the pinwheel annihilation process. Figure 5.12(c) shows the time evolution of the power of OP and OD maps. Pinwheel annihilation starts when the power of the OP map starts to rise which is later in case of inter-map coupling compared to the uncoupled case. Intuitively, a stripe pattern of one map enforces the coevolving map to arrange in stripes such that both run perpendicular to each other. This additional force can accelerate pinwheel annihilation. In these simulations we further identify the limit in which the backreaction onto the OP map can be neglected. With decreasing ratio $r_{o} / r_{z}$ the time evolution of the pinwheel density more and more resembles that of the uncoupled case (red lines in Fig. 5.12).

In the uncoupled case OP maps are pinwheel rich and show a universal pinwheel statistics [16]. In the following we check whether this pinwheel statistics is robust against a small influence of the backreaction from the OD map. We therefore study the time evolution of the pinwheel 
densities as well as the distribution of nearest neighbor pinwheel distances. Figure 5.13 shows the pinwheel statistics in the pinwheel preserving case. In the chosen parameter regime pinwheels are preserved in the uncoupled case (red lines). We use $N=20$ band-pass filtered Gaussian random fields as initial conditions. Inter-map coupling is chosen such that the $n=8$ uniform solutions are stable in the amplitude equations. In Figure 5.13 (a-d) parameters are chosen such that the backreaction onto the OP map is small. We observe hat the final OP maps in case of inter-map coupling are very similar to those in the coupled case. Moreover the pinwheel densities in both cases are very similar. In particular, the type of planform is unaffected by inter-map coupling and also the absolute value and phase of the active modes receive only small corrections. The mean pinwheel density of final states with and without inter-map coupling is given by $\langle\rho\rangle \approx 2.64$. The distribution of nearest neighbor pinwheel distances in the coupled and uncoupled case are almost identical at intermediate times as well as at final times. In Fig. $5.13(\mathrm{e}-\mathrm{h})$ we increased the backreaction onto the OP map. Here, inter-map coupling leads to substantial changes in the final states compared to the uncoupled dynamics. Inter-map coupling in some cases leads to a flip of active modes such that different ECP solutions are realized. In one case inter-map coupling even leads to complete pinwheel annihilation. The average pinwheel density at the final time drops from $\langle\rho\rangle \approx 2.64$ to $\langle\rho\rangle \approx 2.25$. These changes are also reflected by distribution of nearest neighbor pinwheel distances, see Fig. $5.13(\mathrm{~g}, \mathrm{~h})$. Whereas at intermediate times the distance distributions are almost identical for coupled and uncoupled situations, at final times there are small deviations. For instance in case of inter-map coupling there are slightly more long $(d>0.75 \Lambda)$ inter-pinwheel distances.

To summarize, for small backreaction OP patterns with and without inter-map coupling are very similar at all stages of development. Moreover, we observe no deviation from the universal pinwheel statistics of the uncoupled dynamics [16]. For larger backreaction we observe small deviations at final times such as flips of active modes which result in a reduction of the pinwheel density. 
(a)

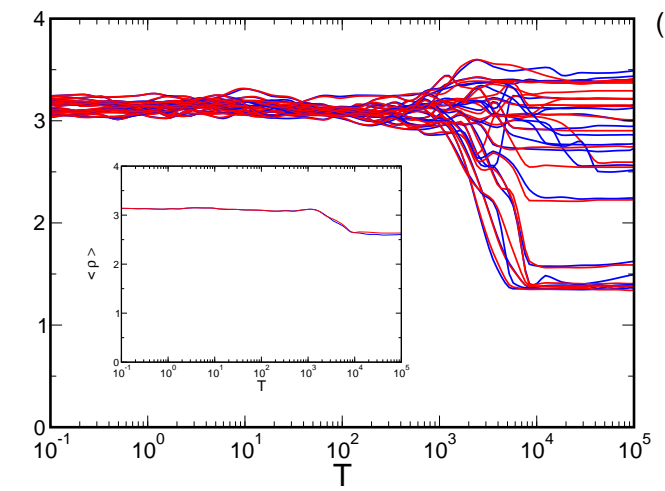

(c)

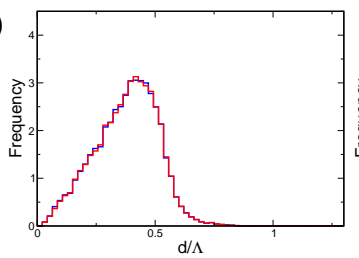

(d)
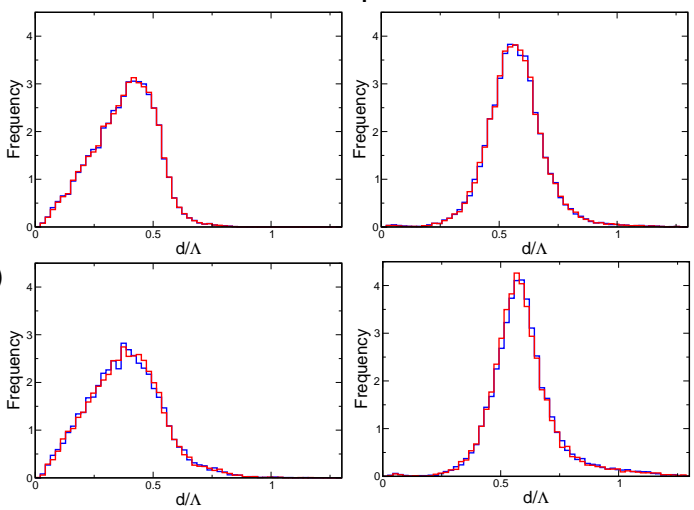

${ }^{(e)} \rho$

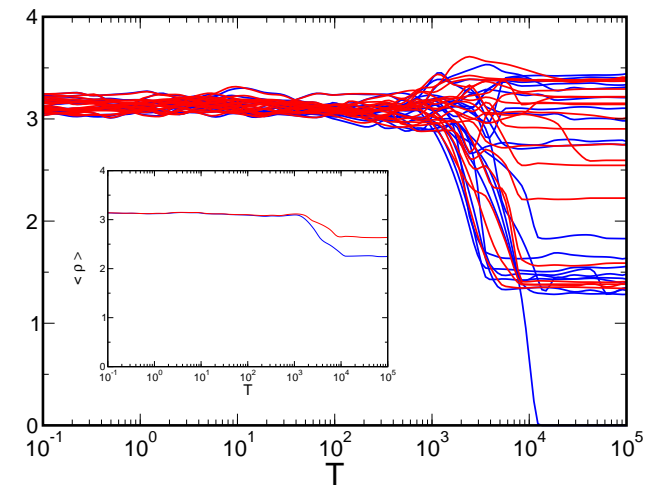

(g)

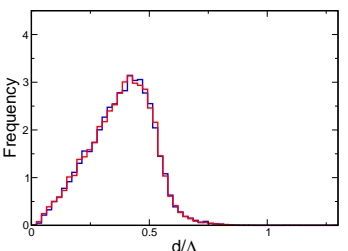

(h)

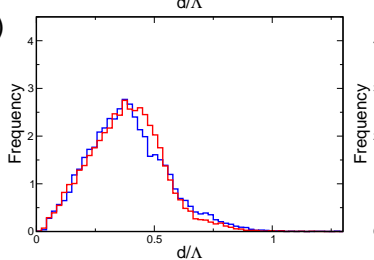

(b)
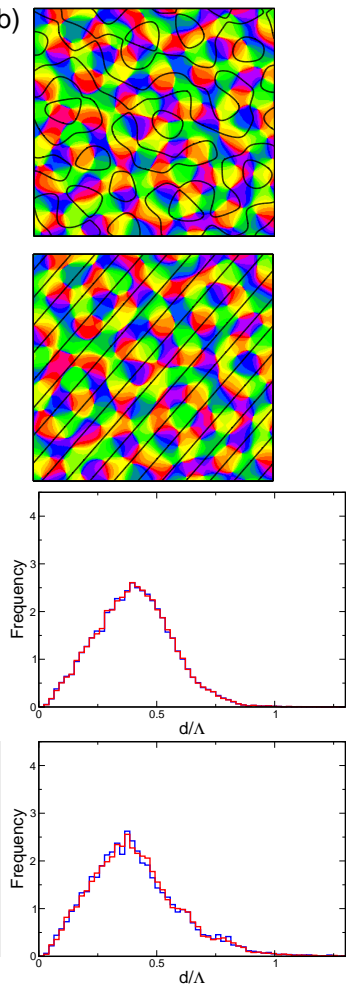

(f)
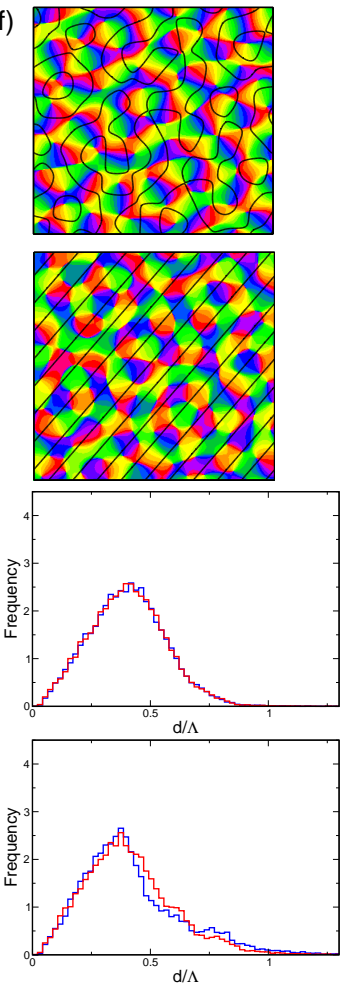

Figure 5.13.: Pinwheel preservation. (a,e) Pinwheel densities in the presence (blue) and absence (red, $\epsilon=0$ ) of inter-map coupling. Inset: mean pinwheel density. (a-d) Small backreaction: $r_{z}=0.1, r_{o}=0.01, \epsilon=5000,(\mathbf{e}-\mathbf{h})$ Larger backreaction:

$r_{z}=0.1, r_{o}=0.05, \epsilon=22000$. (b,f) Examples of final states with and without inter-map coupling. (c,g) Nearest neighbor pinwheel distance of opposite (left), equal (middle), or arbitrary charge (right), $T=300,(\mathbf{d}, \mathbf{h}) T=10^{5}$. Parameters for all simulations: $g=0.98, \Gamma=22, \sigma=1.43 \Lambda, 128 \times 128$ mesh. 


\subsubsection{Time evolution of the OD layout}

We numerically study the time evolution of the OD layout under the influence of intermap coupling. During evolution we track the uniformity parameter $\chi$, Eq. (5.28), and the bandedness $b$ of the OD pattern. Figure [5.14(a,b) shows the time evolution of $N=20$ realizations of OD stripe patterns interacting with $n=4$ (blue) or $n=8$ (red) LDP patterns. The backreaction onto the OP map is small, $r_{o} / r_{z}=0.08$. In case of $n=4$ there is a rapid increase of $\chi$ at $T \approx 1$ which saturates at $\chi$ close to 1 . At about the same time the bandedness decreases to $b \approx 0.05$. The stationary solutions all belong to the $n=4, \Delta=2$ uniform solution class and we can identify the phase relations predicted in Eq. (5.23). In case of $n=8$ the uniformity parameter increases later, at about $T \approx 10$, and the distribution of final $\chi$ values is much broader ranging between $\chi \approx 0.5$ and $\chi \approx 0.9$. Note, that for some simulations $\chi>1$ at $T \approx 100$ which indicates that there are more than eight Fourier modes present in the spectrum but which will afterwards decay. Stationary pattern consist of $n=8, \Delta=2$ and $n=8, \Delta=3$ uniform solutions as well as non-uniform solutions which can explain the broader distribution of the uniformity parameter. Remarkably, the bandedness is as in the case of $n=4$ very small and close to $b \approx 0.05$. In Fig. $5.14(\mathrm{c}, \mathrm{d})$ the simulations are initialized with random fields for the OD and OP map. Here, the bandedness is low already at the initial stage of the time evolution. Without inter-map coupling (green lines) the bandedness rapidly increases at $T \approx 10$ and saturates at $b \approx 0.8$ which corresponds to OD stripe solutions. With inter-map coupling the final OP maps mainly consist of $n=8$ Fig. 5.14(c) and $n=4$ Fig. 5.14(d) ECP solutions. In case of $n=8$ the distribution of the OD bandedness is much more diverse compared to simulations with an OP planform as initial condition. In case of $n=4$ the bandedness stays small and reaches its final value $b \approx 0.05$.

\subsubsection{The OD layout of macaques: From patches to stripes}

So far, we restricted ourselves to the case $k_{c, z}=k_{c, o}$, a condition that is fulfilled in cats [55,79]. In general a detuning of the two wavelengths is possible, a scenario we already studied in Section 4.6.2. As the ratio $k_{c, o} / k_{c, z}$ varies for different species, for instance in macaque monkeys this ratio is $k_{c, o} / k_{c, z} \approx 0.8$ [75], we study how this influences the OD layout. We do not use weakly nonlinear analysis, applied in the case of $k_{c, o}=k_{c, z}$. The resonance conditions are very sensitive to the exact ratio of the wavelengths. With different ratios of the wavelengths, the modes of the patterns are coupled in a different manner. Therefore we restrict ourselves to numerical simulations of the full field dynamics Eq. (5.1).

To reveal the structural changes the OD layout experiences we use a single OP map as initial condition which corresponds to an attractor state in the uncoupled dynamics. The width $\sigma$ is chosen such that planforms with $n=8$ active modes are the dominant solution. As initial conditions for the OD map we use $N=10$ random fields. The bifurcation parameters are such that the backreaction onto the OP map is small. In case of the gradient-type inter-map coupling the coupling strength depends on the wave number $k_{c}$. A change in the critical wavelength therefore also changes the effective coupling between the maps $\tilde{\epsilon}=\epsilon k_{c, z} k_{c, o}$, 
(a)

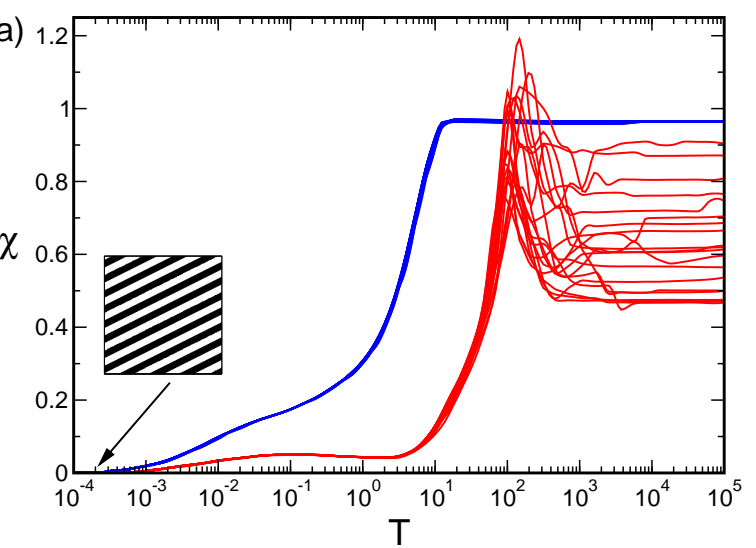

(c)

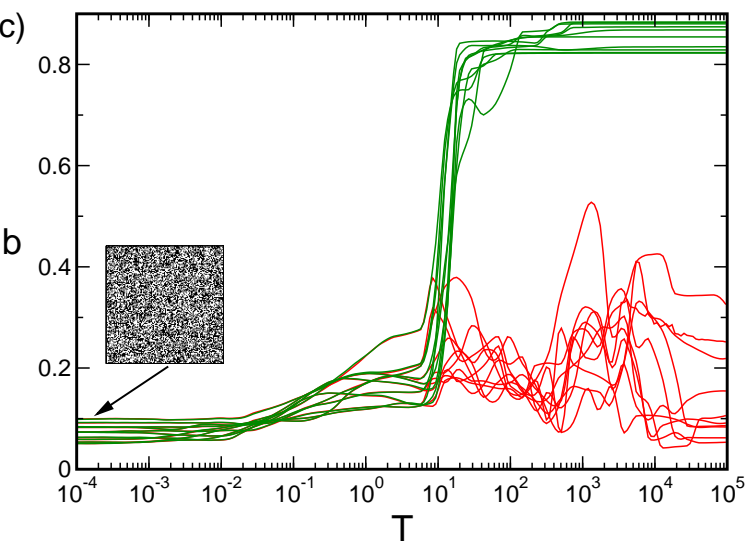

(b)

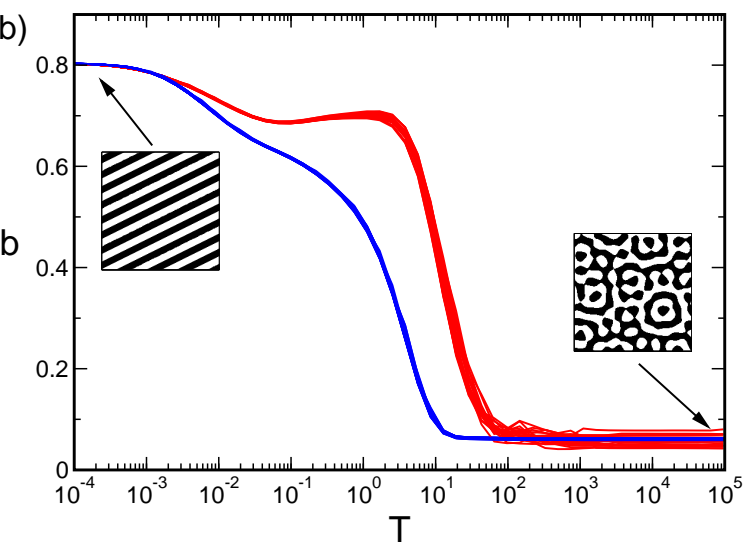

(d)

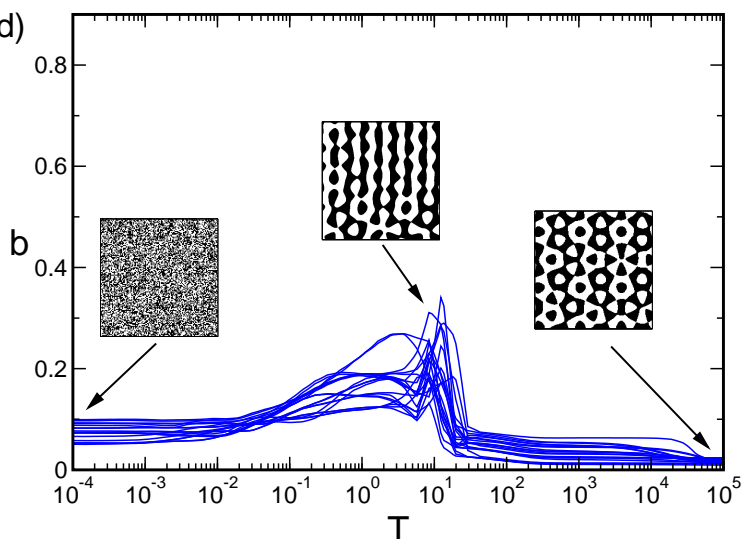

Figure 5.14.: Transition towards uniform solutions. (a,b) OD stripes as initial conditions. (a) Time evolution of the uniformity parameter $\chi$. Blue (red) $\sigma=0.71 \Lambda$ $(\sigma=1.43 \Lambda)$ for which $n=4(n=8)$ planforms dominate. (b) Time evolution of the bandedness $b$. (c,d) Random initial conditions. (c) Green: $\epsilon=0$, red: $\epsilon=2000, \sigma=1.43 \Lambda$ (d) $\epsilon=2000, \sigma=0.71 \Lambda$. Parameters in all simulations: $g=0.98, r_{o}=0.02, r_{z}=0.25, \epsilon=2000, \Gamma=44,256 \times 256$ mesh. Insets: Sign of $o(\mathbf{x})$.

see Eq. (5.18). When changing the wavelength ratio we keep this effective coupling fixed. Figure 5.15 shows the bandedness of the stationary OD map as a function of the ratio $k_{c, o} / k_{c, z}$. We observe that the transfer of spatial irregularity onto the OD pattern is robust against a detuning of the typical wavelengths. There is no ratio for which $b=1$, i.e. for there are regular OD stripes. Instead $b<0.65$ in all examined ratios and the stationary OD maps are spatially irregular. For the ratio $k_{c, z} / k_{c, o}=1$ the OD pattern has a low bandedness and the pattern has a patchy layout similar to those of cats, see Fig. 2.3(a). The standard deviation of the bandedness is rather low. Detuning this ratio in general increases the bandedness. Moreover, the standard deviation of the bandedness increases. For the ratio $k_{c, z} / k_{c, o} \approx 0.82$ the layout is much more stripe-like and comparable to the OD patterns of macaques, see Fig. 2.3(b). Phase relations are important for the stability of patchy OD solutions. As the two critical circles for $k_{c, o} \neq k_{c, z}$ do not overlap we expect different or even absent phase relations and therefore a more stripe-like layout. Increasing the inter-map coupling does not change this result, see inset in Fig. 5.15(b). However, if we increase the bifurcation parameter $r_{o}$, see Fig. 5.15(b), it becomes more likely to obtain resonant combinations of active modes 


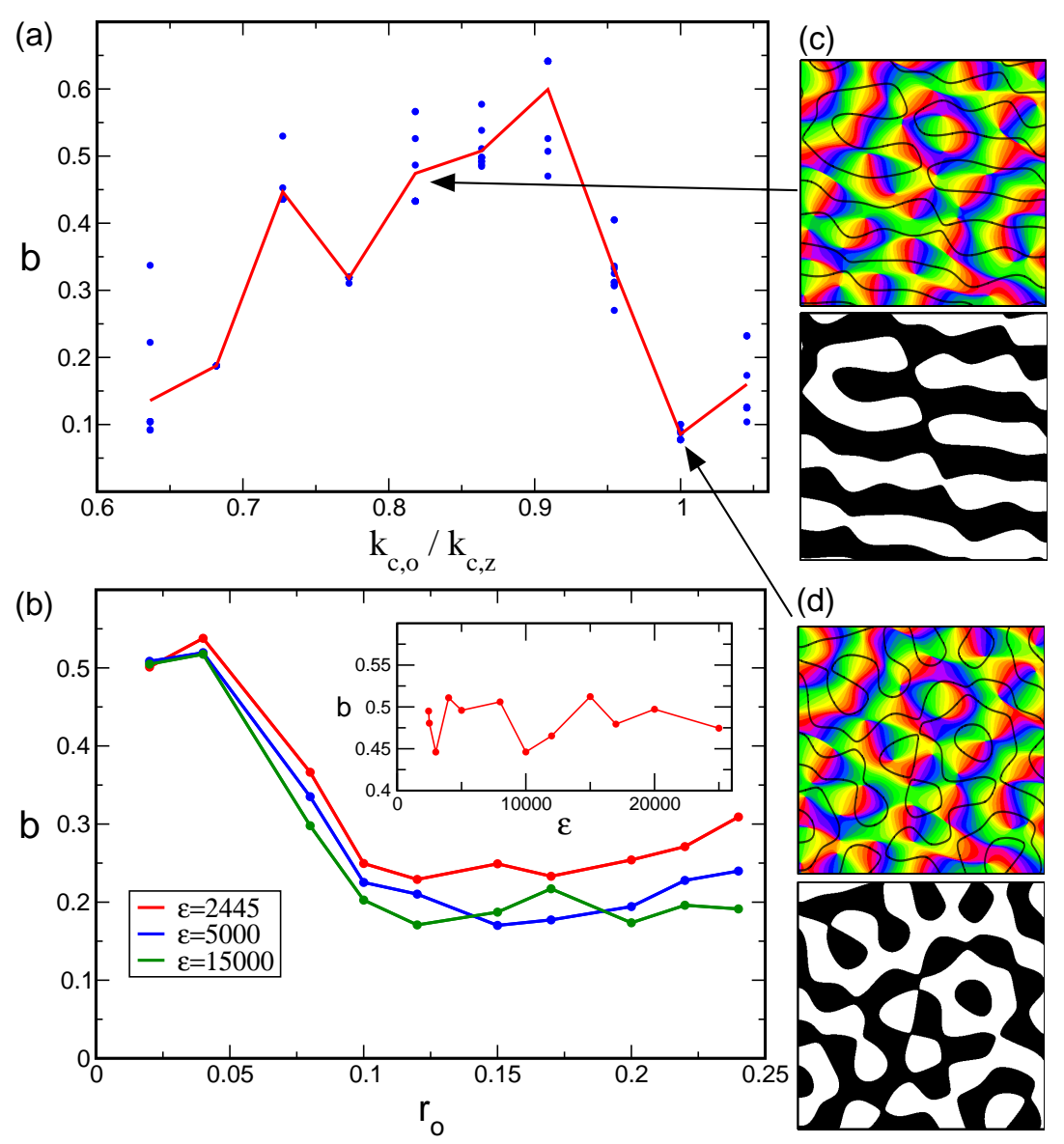

Figure 5.15.: Layout differences when detuning OD and OP wavelengths. (a) Bandedness $b$ for different ratios $k_{c, o} / k_{c, z}$. Red line: mean bandedness. Parameters for all simulations: $g=0.98, \sigma=1.43 / \Lambda, r_{z}=0.25, r_{o}=0.02, \epsilon=2000 / k_{c, o}, T_{f}=5 \cdot 10^{5}$, mesh $=128 \times 128$. (b) Bandedness for the fixed ratio $k_{c, o} / k_{c, z}=18 / 22 \approx 0.82$ and varying $r_{o}$ and $\epsilon$ (inset, $r_{o}=0.02$ ). Parameters: $g=0.98, \sigma=1.43 \Lambda, r_{z}=0.25$. (c,d) Typical pattern of OD and OP map for (c) $k_{c, o} / k_{c, z} \approx 0.82$ or (d) $k_{c, o} / k_{c, z}=1$.

which are located in a region with a positive growth rate. 


\subsection{Discussion}

In this chapter we studied a complementary realization of the general model Eq. (3.1). In particular, we demonstrated that the layout of OD maps can be influenced by inter-map coupling. We showed that inter-map coupling leads to a transition from regular OD stripes towards a spatially aperiodic OD pattern which resembles the patchy OD layout found in cat visual cortex. Similar to the previous chapter we studied the coupled dynamics close to the pattern formation threshold, using weakly nonlinear analysis to derive amplitude equations. As in the previous chapter we identified a limit in which inter-map coupling becomes unidirectional and further demonstrated that, when using the higher order inter-map coupling energies, we could neglect the backreaction onto the OP map. Notably, this limit $r_{o} \ll r_{z}$ is opposite to the limit applied in the previous chapter where $r_{z} \ll r_{o}$. We have shown in this chapter that the condition $r_{o} \ll r_{z}$ is not necessary for the stability of uniform solutions which can become stable even for $r_{o} \approx r_{z}$. However, this limit enables us to neglect the backreaction on the OP map which substantially simplifies the analysis of map interactions. Moreover, we demonstrated that the quantitative properties of the uncoupled OP solutions [16] are preserved for small backreaction.

In this limit we analytically studied the existence and stability properties of uniform solutions, spatially irregular solutions which resemble experimentally obtained OD and OP maps and which can be considered as generalizations of the PWC solutions discussed in the previous chapter. We further studied the changes in the stability properties due to the influence of the backreaction on the OP map. We showed that a finite backreaction shifts the stability borders but leaves the qualitative properties of the phase diagrams unaffected.

We gave a potential explanation for the OD layout differences observed in cat and monkey visual cortex. These species show a striking dissimilarity in their OD layout which is patchy in the case of cats, while more banded in the case of macaque monkeys. We showed that such layout differences can be explained by differences in the average wavelength of the two patterns. In contrast to cat visual cortex where both wavelengths are equal [55, 79], in macaque monkeys the OD map has a larger average wavelength than the OP map [56, 75]. We thus detuned these wavelengths in the coupled Swift-Hohenberg equations. We showed that if the wavelength of the OD map equals that of the OP map, the OD layout obtained by inter-map coupling is patchy and resembles that found in cats. However, when the wavelength of the OD map is larger than that of the OP map, as it was observed in macaque monkeys, the resulting OD layout is more banded, resembling that of macaques. A similar connection between the wavelength differences of both patterns and the OD layout has been drawn in 99. Pattern formation of such a form has been previously studied for instance in Faraday waves [6,, 106 , using a single order parameter field but two different critical wavelengths. The presented scenario can be generalized to other species such as ferrets, in which the wavelength of the OD map is smaller than that of the OP map [63, 107].

In this chapter we showed that the analyzed inter-map coupling energies can lead to realistic geometric relationships between OD and OP maps. We quantified the geometric relationships 
of the stable solutions obtained by amplitude equations as well as from solving the full field dynamics numerically. The gradient-type coupling energy is sensitive to intersection angles between iso-orientation lines and OD borders. This is also reflected by the resulting solutions of the dynamics. In all cases we observe a tendency towards perpendicular intersection angles. This tendency is more pronounced for LDP solutions, where the OP pattern is more anisotropic, than for HDP solutions. With an increasing number of active modes the pattern become more irregular and the bias for perpendicular angles is less pronounced. The observed continuous distributions resemble those found in experiments [30,68, 73, 175, 108, [110]. In case of the product-type coupling energy, however, the distribution of intersection angles clearly differs from that of the gradient-type energy. For a low number of active modes we obtained a second peak at a parallel angle whereas for higher numbers of active modes the distribution becomes flat. In contrast to the previous chapter, for spatially irregular OP maps the used coupling energies indeed have an impact on the distribution of intersection angles.

Next, we quantified the distribution of pinwheel positions with respect to OD. Experimentally, the tendency for pinwheel centers to be located at OD centers has been reported and quantified in a variety of experiments $[36,68,73,74,108,109,111]$. For most stable uniform solutions we found a clear tendency for pinwheels to be located at OD extrema. However, we also found solutions in which the distribution is flat. In the case of solutions of the full field dynamics we observe in all cases a tendency for pinwheels to be at OD extrema. Differences to results from amplitude equations might results from stable non-uniform solutions and from modulations in the stationary amplitudes which appear far from threshold.

In some previous models the timing of map development is an important factor in determining the resulting map layout [27, 112, 113. If the OD map is coupled to an already established system of OP columns the resulting OD layout is patchy. The distribution of intersection angles has also been related to the developmental order of the maps where the distribution is more biased if OP maps develop before OD maps [28]. However, as the precise developmental order is still unknown and these models furthermore lead to pinwheel annihilation [18, we did not include such an order in our model. In the previous chapter we demonstrated that inter-map coupling can solve the problem of pinwheel stability. It has been found in several studies [18, 20] that inter-map coupling slows down pinwheel annihilation. In contrast, we found that in a regime where pinwheels are unstable, the process of pinwheel annihilation is slightly accelerated by inter-map coupling.

In this chapter we presented a model leading to spatially irregular OP and OD maps. This has been achieved by introducing non-local interactions to the coupled dynamics. These interaction terms were motivated by the observed long-range horizontal connections which have been found in a variety of species [39 48]. We introduced long-range interactions for the OP part of the dynamics only. Whereas long-range connections are sensitive to orientation selectivity, their relationship to OD is less clear. It has been shown that the inclusion of such terms can lead to spatially aperiodic OP maps [49,50]. An important property of the uncoupled OP dynamics is the existence of a huge number of multistable solutions. Inter-map coupling can transfer this multistability leading to numerous patchy OD patterns. 


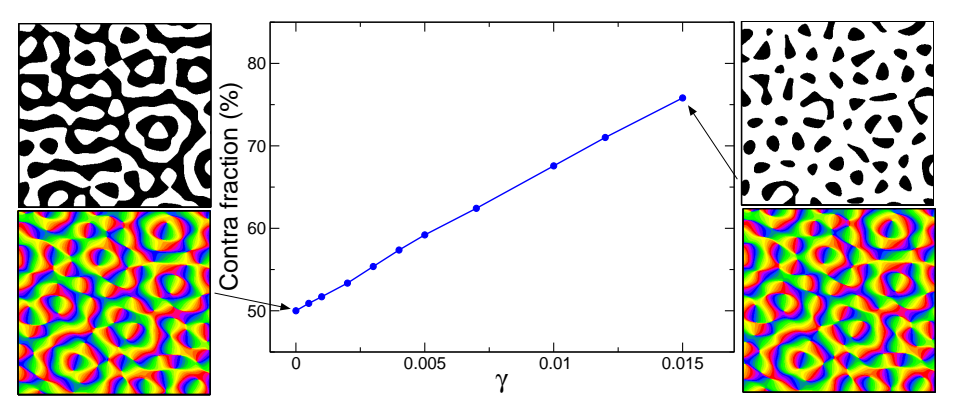

Figure 5.16: Monocular deprivation. Contra fraction, see Section 4.2.5, of final states with OD bias and long-range interactions. Parameters: $r_{z}=0.25, r_{o}=0.04, g=0.98$ $\sigma=1.43 \Lambda, \epsilon=2000, T_{f}=10^{5}$, $\Gamma=22,128 \times 128$ mesh.

In species that lack OP columns our model predicts regular OD stripes. It is hard to examine such a scenario, as many species such as rodents that lack OP columns also show no OD segregation [76]. One possibility to investigate OD columns without the influence of OP is to artificially induce an OD map. This has been done experimentally in tadpoles [86, 114, implanting a third eye, as well as in theoretical studies [115]. The resulting OD map in the optic tectum of the tadpole is indeed banded, supporting our notion that without inter-map coupling the OD map is a stripe pattern.

In this chapter we have not included an eye-inversion symmetry breaking term as in the previous chapter. With the inclusion of such a bias we can study for instance the effect of monocular deprivation on the patchy layout of OD maps. Final states of such simulations are shown in Fig. 5.16. Shown is also the percentage of contralateral eye dominated territory, see also Section 4.2.5. In the case of OD stripes and hexagons there is a gap in the realized fraction of contralateral eye dominance, see Fig. 4.2(b). With the inclusion of long-range interactions, however, we obtained a continuous distribution such that all possible fractions of eye dominance can be realized.

To conclude, the approach presented in this chapter demonstrates that a realistic map layout of diverse species such as cats and macaques can be obtained by inter-map coupling. In the following chapters we investigate the consequences of the degeneracy of OP solutions and discuss pattern selection when this degeneracy is lifted. 


\section{Pattern Selection Far from Threshold}

\subsection{A preliminary remark}

The inclusion of non-local interactions into the OP dynamics leads to stable, spatially aperiodic patterns which resemble physiological maps as demonstrated in the last chapter. This dynamics, however, has a vast number of stationary solutions. The enormous complexity of this model becomes apparent by the fact that the number of stationary solutions grows exponentially with the number of active modes $n$. Nevertheless, this model is relatively easy to handle due to the existence of permutation symmetry and the assumption that pattern formation takes place close to threshold. Here, all solutions for a given $n$ share the same stability and energy properties and thus all solutions can be treated in a common fashion. Multistability reflects the situation found in the visual cortex where different observed patterns are never identical but only qualitatively similar. In this and the following chapter we study mechanisms that can lift the energetic degeneracy. In this case every single solution has to be treated individually. We thus present a comprehensive characterization of the large class of stationary solutions. What do we expect if the described degeneracy is lifted? Every single solution will receive its own stability borders and eventually the stationary amplitudes and phases of the solutions itself become non-trivial and planform dependent. We further expect an energetic selection of particular solutions. The energy landscape might change such that the basin of attraction of particular solutions is increased. Even new classes of stationary solutions can become relevant which were unimportant before. In the following chapters we describe all of these phenomenons and in particular how they relate to the resulting pinwheel densities. Moreover, we give a thorough characterization of the full richness of solution classes of the OP dynamics.

If the presented model is very sensitive to the distance from threshold or to deviations from permutation symmetry its tractability would be of limited value. In the following we show that this is not the case and that the OP dynamics introduced in the previous chapter to a large extend is insensitive to such model details.

\subsection{Introduction}

The previous chapters demonstrated that weakly nonlinear analysis is a powerful tool to analytically examine the selection of patterns due to inter-map coupling. The amplitude equations of the uncoupled OP dynamics, when truncated at leading order, show multistability and energetic degeneracy of different planform solutions. The pinwheel density averaged over 
the different multistable solutions approaches $\rho=\pi$ for large interaction width $\sigma$. However, numerical simulations of the full field dynamics indicate that the energetic degeneracy is lifted far from threshold. With increasing distance from threshold the average pinwheel density of the stationary patterns decreases substantially below $\rho=\pi$. To characterize this distance dependence we consider higher order corrections to the uncoupled OP amplitude equations. We show that fifth order corrections lead to modulations in the stationary amplitudes which in general decrease the pinwheel density of the corresponding planform solution. Moreover, higher order corrections energetically select those planform solutions which have a relatively low pinwheel density. These results thus can explain the observed decrease in the pinwheel density. Higher order corrections not only affect the amplitudes of the Fourier modes. We show that for certain planform configurations also the degeneracy of their phases is partly lifted. Lifting the degeneracy of phases additionally lowers the corresponding potential leading to an over-representation of these solutions in the phase diagram. We begin this chapter with the derivation of amplitude equations beyond the leading order.

\subsection{Amplitude equations - Higher order corrections}

In this section we derive amplitude equations up to fifth order of the uncoupled OP dynamics and demonstrate that these corrections lift the degeneracy of ECP solutions. We first consider an OP dynamics with general cubic and quintic nonlinearities $N_{3}, N_{5}$ given by

$$
\partial_{t} z(\mathbf{x}, t)=\hat{L} z(\mathbf{x}, t)-N_{3}[z, z, \bar{z}]-N_{5}[z, z, z, \bar{z}, \bar{z}]
$$

with $\hat{L}=r-\left(k_{c}^{2}+\Delta\right)^{2}=r-\hat{L}^{0}$. This dynamics is then specified where $N_{3}$ is given in Eq. (3.4) and $N_{5}=0$. The expansion up to third order is detailed in Section 4.3. We have seen that due to orientation shift symmetry all even terms in the expansion vanish. Therefore we expand the field in powers of $\mu=\sqrt{r}$ as

$$
z=\mu z_{1}+\mu^{3} z_{3}+\mu^{5} z_{5}+\ldots
$$

In addition, we introduce a slow timescale $T=r t$. We insert the expansion in the dynamics Eq. (6.1) and get

$$
\begin{aligned}
0 & =\mu \hat{L}^{0} z_{1} \\
& +\mu^{3}\left(-\partial_{T} z_{1}+z_{1}-\hat{L}^{0} z_{3}-N_{3}\left[z_{1}, z_{1}, \bar{z}_{1}\right]\right) \\
& +\quad \mu^{5}\left(-\partial_{T} z_{3}+z_{3}-\hat{L}^{0} z_{5}-N_{3}\left[z_{1}, z_{1}, \bar{z}_{3}\right]-N_{3}\left[z_{1}, z_{3}, \bar{z}_{1}\right]-N_{3}\left[z_{3}, z_{1}, \bar{z}_{1}\right]\right. \\
& \left.\quad-N_{5}\left[z_{1}, z_{1}, z_{1}, \bar{z}_{1}, \bar{z}_{1}\right]\right)
\end{aligned}
$$


Besides the leading order homogeneous equation $\hat{L}^{0} z_{1}=0$ with the solution

$$
z_{1}=\sum_{j=0}^{n-1} A_{j}(T) e^{i \vec{k}_{j} \vec{x}}+\sum_{j=0}^{n-1} A_{j^{-}}(T) e^{-i \vec{k}_{j} \vec{x}}
$$

we get, at subsequent orders of $\mu$, inhomogeneous equations of the form

$$
\hat{L}^{0} z_{m}=F_{m}=z_{m-1}-\partial_{T} z_{m-1}-N_{3}\left[z_{1}, \ldots, z_{m-1}\right]-\ldots
$$

arise. At every order $\mu$ the inhomogeneous equation is solved in two steps. The nonlinear terms involve combinations of the original Fourier modes Eq. (6.4). These combinations are divided into two classes. For those modes that have their wavevector on the critical circle we can apply the solvability condition. By satisfying the solvability condition all modes on the critical circle are removed. In the second step the modes with wavevectors off the critical circle $\vec{k}_{s},\left|\overrightarrow{k_{s}}\right| \neq k_{c}$ are considered. In this case we can invert the linear operator

$$
\left(\hat{L}^{0}\right)^{-1}=\frac{-1}{\left(k_{c}^{2}+\Delta\right)^{2}}
$$

If we apply the operator $\left(\hat{L}^{0}\right)^{-1}$ to modes off the critical circle we obtain

$$
\left(\hat{L}^{0}\right)^{-1} e^{\imath \vec{k}_{s} \vec{x}}=\frac{-1}{\left(k_{c}^{2}-k_{s}^{2}\right)^{2}} e^{\imath \vec{k}_{s} \vec{x}}
$$

and therefore equation Eq. (6.5) can be inverted leading to

$$
z_{m}=\left(\hat{L}^{0}\right)^{-1} F_{m}=\hat{L}^{-1}\left(-N_{3}\left[z_{1}, \ldots, z_{m-1}\right]-N_{5}\left[z_{1}, \ldots, z_{m-1}\right]\right)
$$

Note, that the inverse operator $\left(\hat{L}^{0}\right)^{-1}$ is acting on nonresonant terms only. The third order equation is given by

$$
\hat{L}^{0} z_{3}=-z_{1}+\partial_{T} z_{1}+N_{3}\left(z_{1}, z_{1}, \bar{z}_{1}\right) .
$$

The solvability condition leads to the following equation at order $\epsilon^{3}$

$$
\left\langle\tilde{z} \mid-z_{1}+\partial_{T} z_{1}+N_{3}\left(z_{1}, z_{1}, \bar{z}_{1}\right)\right\rangle=0 ; \hat{L}^{0} \tilde{z}=0 .
$$

We introduce the operator $\hat{P}_{c}$ which projects onto the kernel of $\hat{L}^{0}$. This leads to

$$
\partial_{T} z_{1}=z_{1}-\hat{P}_{c} N_{3}\left[z_{1}, z_{1}, \bar{z}_{1}\right]
$$

We insert the leading order solution Eq. (6.4) and get

$$
\partial_{T} A_{i}=A_{i}+\hat{P}_{i} \sum_{j, k, l} A_{j} A_{k} \bar{A}_{l} e^{-\imath \vec{k}_{i} \vec{x}} N_{3}\left[e^{\imath \vec{k}_{j} \vec{x}}, e^{\imath \vec{k}_{k} \vec{x}}, e^{-\imath \vec{k}_{l} \vec{x}}\right]
$$


where $\hat{P}_{i}$ is the projection operator onto the subspace $\left\{e^{2 \vec{k}_{i} \vec{x}}\right\}$ of the kernel. This leads to the amplitude equations at leading order

$$
\partial_{t} A_{i}=r A_{i}-\sum_{j}^{n} g_{i j}\left|A_{j}\right|^{2} A_{i}-\sum_{j}^{n} f_{i j} A_{j} A_{j^{-}} \bar{A}_{i^{-}},
$$

where we scaled back to the original time variable $t$.

Following the expansion Eq. (6.3) and using Eq. (6.8) we get the third order field field as

$$
z_{3}=\left(\hat{L}^{0}\right)^{-1} N_{3}\left(z_{1}, z_{1}, \bar{z}_{1}\right)+\sum_{j}^{n} B_{j} e^{\imath \vec{k}_{j} \vec{x}}+\sum_{j}^{n} B_{j}-e^{-\imath \vec{k}_{j} \vec{x}} .
$$

For the general solution we also added a solution of the homogeneous equation i.e. modes $B_{j}$ and $B_{j^{-}}$on the critical circle. The equation to be solved at fifth order is given by

$$
\begin{gathered}
\hat{L}^{0} z_{5}=-z_{3}+\partial_{T} z_{3}+N_{3}\left(z_{1}, z_{1}, \bar{z}_{3}\right)+N_{3}\left(z_{3}, z_{1}, \bar{z}_{1}\right)+N_{3}\left(z_{1}, z_{3}, \bar{z}_{1}\right) \\
+N_{5}\left(z_{1}, z_{1}, z_{1}, \bar{z}_{1}, \bar{z}_{1}\right) .
\end{gathered}
$$

Inserting Eq. (6.14) and Eq. (6.4) and applying the solvability condition leads to the following amplitude equations

$$
\begin{aligned}
\partial_{T} B_{i}= & B_{i}-\sum_{j}^{n} g_{i j}\left(\left|A_{j}\right|^{2} B_{i}+A_{i} \bar{A}_{j} B_{j}+A_{i} A_{j} \bar{B}_{j}\right) \\
& -\sum_{j}^{n} f_{i j}\left(\bar{A}_{i^{-}} A_{j^{-}} B_{j}+\bar{A}_{i^{-}} A_{j} B_{j^{-}}+A_{j} A_{j^{-}} \bar{B}_{i^{-}}\right) \\
& -\sum_{j, k}^{n} g_{i j k}\left|A_{j}\right|^{2}\left|A_{k}\right|^{2} A_{i} \\
& -\sum_{j, k}^{n} \tilde{f}_{i j k} \bar{A}_{k} \bar{A}_{k^{-}} A_{j} A_{j^{-}} A_{i}-\sum_{j, k}^{n} f_{i j k}\left|A_{k}\right|^{2} A_{j} A_{j^{-}} \bar{A}_{i^{-}},
\end{aligned}
$$

and corresponding equations for the modes $B_{i^{-}}$. The coupling coefficients of the third order terms are given in Eq. (4.59) while the fifth order coupling terms terms are given in Appendix A.5. We can insert the stationary solution for the leading order equation Eq. (6.13) into Eq. (6.16). This leads to a linear equation for the fifth order correction $B_{i}$ and $B_{i^{-}}$. From the leading order we already know that the amplitudes of the ECP solutions are uniform and degenerate in their phases. Furthermore their opposite modes are suppressed and we can set $A_{i}=\mathcal{A}, A_{i^{-}}=0$. This leads to

$$
\begin{aligned}
\partial_{T} B_{i} & =B_{i}-\sum_{j} g_{i j} \mathcal{A}^{2}\left(B_{i}+B_{j}+\bar{B}_{j}\right)-\sum_{j, k} g_{i j k} \mathcal{A}^{5} \\
\partial_{T} B_{i^{-}} & =B_{i^{-}}-\sum_{j} g_{i j^{-}}|A|^{2} B_{i^{-}}-\mathcal{A}^{2} \sum_{j} f_{i j} B_{j^{-}} .
\end{aligned}
$$


Remarkably, the dynamics of the modes $B_{i^{-}}$does not contain quintic interaction terms, as is the case for the dynamics of the modes $B_{i}$. Using the third order stationarity condition $\left(1-\sum_{j} g_{i j} A^{2}\right)=0$ we obtain

$$
\begin{aligned}
\partial_{t} B_{i} & =-2 \sum_{j} g_{i j} \mathcal{A}^{2} B_{j}-\sum_{j, k} g_{i j k} A^{5} \\
\partial_{t} B_{i^{-}} & =-\mathcal{A}^{2} \sum_{j} f_{i j} B_{j^{-}} .
\end{aligned}
$$

The stationarity condition results in an inhomogeneous and a homogeneous linear equation. The homogeneous equation has the trivial solution $B_{i^{-}}=0$ which is the only unique solution. Moreover, if the eigenvalues of $f_{i j}$ are nonzero this is even the only solution. As $f_{i j}$ is a circulant matrix its eigenvalues can be calculated easily. For $\sigma$ large the eigenvalues are given by $(n-1) g / 2$ and $-g / 2$. Therefore the modes $B_{i^{-}}$receive no fifth order corrections. This situation changes if we consider configurations of active modes that allow for triad resonances, see Section 6.4.

We can combine the third and fifth order contributions by introducing $\tilde{A}_{j}=\mu A_{j}+\mu^{3} B_{j}$, $\tilde{A}_{j^{-}}=\mu A_{j^{-}}+\mu^{3} B_{j^{-}}$and collect all contributions up to $\mu^{5}$. After rescaling back to the fast time variable and rewriting $\tilde{A}_{i} \rightarrow A_{i}$ we get the fifth order amplitude equations

$$
\begin{aligned}
\partial_{t} A_{i}= & r A_{i}-\sum_{j}^{n} g_{i j}\left|A_{j}\right|^{2} A_{i}-\sum_{j}^{n} f_{i j} A_{j} A_{j} \bar{A}_{i^{-}}-\sum_{j, k}^{n} g_{i j k}\left|A_{j}\right|^{2}\left|A_{k}\right|^{2} A_{i} \\
& -\sum_{j, k}^{n} f_{i j k}\left|A_{k}\right|^{2} A_{j} A_{j-} \bar{A}_{i-}-\sum_{j, k}^{n} \tilde{f}_{i j k} \bar{A}_{k} \bar{A}_{k-} A_{j} A_{j-} A_{i} .
\end{aligned}
$$

The amplitude equations (6.19) equals Eq. (6.17) together with Eq. (6.13) up to a correction of the order $\mu^{7}$. As for the third order amplitude equations, if opposite modes are suppressed $A_{j^{-}}=0$ the amplitudes are degenerate in their phases. Again the coupling coefficients can be expressed as an angle dependent function. At fifth order there are interactions between three active modes and we thus define two angles $\alpha=\left|\alpha_{i}-\alpha_{j}\right|$ and $\beta=\left|\alpha_{i}-\alpha_{k}\right|$. The coupling 

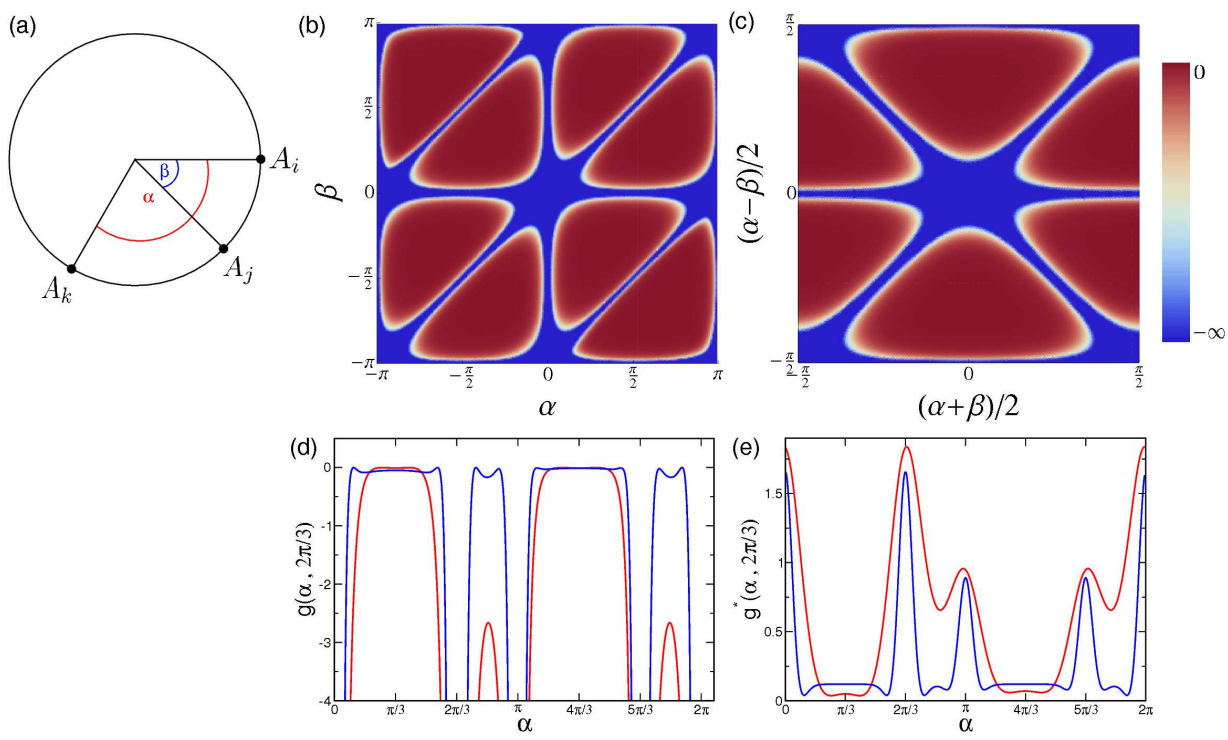

Figure 6.1.: Fifth order coupling function. (a) Illustration of interactions between three modes that contribute to $g_{i j k}$. (b,c) Coupling function $g(\alpha, \beta)$, see Eq. (6.20), with $g=0.9, \sigma=0.4 \Lambda$. (d) Section through the coupling function $g(\alpha, \beta=2 \pi / 3)$, red:

$g=0.9, \sigma=0.4 \Lambda$, blue: $g=0.9, \sigma=0.9 \Lambda$. The coupling function diverges at $\alpha, \beta=j \pi$ and $\alpha=\beta+j \pi, j=0,1, \ldots$ (e) Coupling function $g^{*}(\alpha, \beta=2 \pi / 3)$ with the linear operator set to the identity, $\hat{L}^{0}=\mathbf{1}$.

function therefore reads

$$
\begin{aligned}
& g(\alpha, \beta)=e^{-\imath \vec{k}_{0} \vec{x}}\left(N_{3}\left[\left(\hat{L}^{0}\right)^{-1} N_{3}\left[e^{\imath \vec{k}_{0} \vec{x}}, e^{\imath h(\alpha) \vec{x}}, e^{-\imath h(\beta) \vec{x}}\right], e^{\imath h(\beta) \vec{x}}, e^{-\imath h(\alpha) \vec{x}}\right]+\right. \\
& N_{3}\left[\left(\hat{L}^{0}\right)^{-1} N_{3}\left[e^{\imath h(\alpha) \vec{x}}, e^{\imath k_{0} \vec{x}}, e^{-\imath h(\beta) \vec{x}}\right], e^{\imath h(\beta) \vec{x}}, e^{-\imath h(\alpha) \vec{x}}\right]+ \\
& N_{3}\left[\left(\hat{L}^{0}\right)^{-1} N_{3}\left[e^{\imath k_{0} \vec{x}}, e^{\imath h(\beta) \vec{x}}, e^{-\imath h(\alpha) \vec{x}}\right], e^{\imath h(\alpha) \vec{x}}, e^{-\imath h(\beta) \vec{x}}\right]+ \\
& N_{3}\left[\left(\hat{L}^{0}\right)^{-1} N_{3}\left[e^{\imath h(\beta) \vec{x}}, e^{\imath k_{0} \vec{x}}, e^{-\imath h(\alpha) \vec{x}}\right], e^{\imath h(\alpha) \vec{x}}, e^{-\imath h(\beta) \vec{x}}\right]+ \\
& N_{3}\left[e^{\imath h(\beta) \vec{x}},\left(\hat{L}^{0}\right)^{-1} N_{3}\left[e^{\imath k_{0} \vec{x}}, e^{\imath h(\alpha) \vec{x}}, e^{-\imath h(\beta) \vec{x}}\right], e^{-\imath h(\alpha) \vec{x}}\right]+ \\
& N_{3}\left[e^{\imath h(\alpha) \vec{x}},\left(\hat{L}^{0}\right)^{-1} N_{3}\left[e^{\imath k_{0} \vec{x}}, e^{\imath h(\beta) \vec{x}}, e^{-\imath h(\alpha) \vec{x}}\right], e^{-\imath h(\beta) \vec{x}}\right]+ \\
& N_{3}\left[e^{\imath h(\beta) \vec{x}},\left(\hat{L}^{0}\right)^{-1} N_{3}\left[e^{\imath h(\beta) \vec{x}}, e^{\imath \vec{k}_{i} \vec{x}}, e^{-\imath h(\beta) \vec{x}}\right], e^{-\imath h(\alpha) \vec{x}}\right]+ \\
& N_{3}\left[e^{\imath h(\alpha) \vec{x}},\left(\hat{L}^{0}\right)^{-1} N_{3}\left[e^{\imath h(\beta) \vec{x}}, e^{\imath k_{0} \vec{x}}, e^{-\imath h(\alpha) \vec{x}}\right], e^{-\imath h(\beta) \vec{x}}\right]+ \\
& N_{3}\left[e^{\imath h(\alpha) \vec{x}}, e^{\imath h(\beta) \vec{x}},\left(\hat{L}^{0}\right)^{-1} N_{3}\left[e^{-\imath h(\alpha) \vec{x}}, e^{-\imath h(\beta) \vec{x}}, e^{\imath k_{0} \vec{x}}\right]\right]+ \\
& \left.N_{3}\left[e^{\imath h(\beta) \vec{x}}, e^{\imath h(\alpha) \vec{x}},\left(\hat{L}^{0}\right)^{-1} N_{3}\left[e^{-\imath h(\beta) \vec{x}}, e^{-\imath h(\alpha) \vec{x}}, e^{\imath k_{0} \vec{x}}\right]\right]\right)+ \\
& N_{3}\left[e^{\imath h(\alpha) \vec{x}}, e^{\imath h(\beta) \vec{x}},\left(\hat{L}^{0}\right)^{-1} N_{3}\left[e^{-\imath h(\beta) \vec{x}}, e^{-\imath h(\alpha) \vec{x}}, e^{\imath k_{0} \vec{x}}\right]\right]+ \\
& \left.N_{3}\left[e^{\imath h(\beta) \vec{x}}, e^{\imath h(\alpha) \vec{x}},\left(\hat{L}^{0}\right)^{-1} N_{3}\left[e^{-\imath h(\alpha) \vec{x}}, e^{-\imath h(\beta) \vec{x}}, e^{\imath k_{0} \vec{x}}\right]\right]\right) \text {, }
\end{aligned}
$$

with $k_{0}=(1,0) k_{c}$ and $h(\alpha)=(\cos \alpha, \sin \alpha) k_{c}$. Note, that $g(\alpha, \beta)=g(\beta, \alpha)$. The coupling coefficients are then obtained by $g_{i j k}=g\left(\left|\alpha_{i}-\alpha_{j}\right|,\left|\alpha_{i}-\alpha_{k}\right|\right), g_{i j j}=\frac{1}{4} g\left(\left|\alpha_{i}-\alpha_{j}\right|,\left|\alpha_{i}-\alpha_{j}\right|\right)$ and $g_{i i j}=\frac{1}{2} g\left(\left|\alpha_{i}-\alpha_{j}\right|, 0\right)$. The coupling function $g(\alpha, \beta)$ is plotted in Fig. 6.1. The function $g(\alpha, \beta)$ is not bounded from below since singularities appear at $\alpha, \beta=j \pi$ and $\alpha=\beta+j \pi, j=$ $0,1, \ldots$ How is the multistability of ECP solutions affected by higher order corrections? The third order coupling function $g(\alpha)$ is $\pi$-periodic due to permutation symmetry which leads to 
multistability. This property is not preserved at fifth order where $g(\alpha+\pi, \beta) \neq g(\alpha, \beta)$ even for permutation symmetric nonlinearities. From this property we can expect that multistability of different planform solutions is lifted at fifth order. The reason for this is twofold. First, contributions from $\left(\hat{L}^{0}\right)^{-1}$ turn out to be planform dependent. This will be detailed in Section 6.8. Second, even if we omit those non-resonant terms, the resulting coupling function is also not $\pi$-periodic. This is illustrated in Fig. 6.1(e) where we set the linear operator to the identity $\hat{L}^{0}=\mathbf{1}$ leading to the coupling function $g^{*}(\alpha, \beta)$ with $g^{*}(\alpha+\pi, \beta) \neq g^{*}(\alpha, \beta)$. Thus the ECP degeneracy is lifted by the coupling function at fifth order.

In the following, we discuss the stationary solutions of Eq. (6.19) and their stability and energy properties. Note, for some planform configurations there are additional contributions to the amplitude equations which will be discussed in Section 6.4

\subsubsection{Examples: Stripes and squares}

In case of stripes $(n=1)$ higher order corrections are absent $\left(g_{i i i}=0\right)$ due to orientation shift symmetry. Indeed, the third order solution $z_{1}=\mathcal{A} e^{\imath\left(k_{c} x+\phi\right)}$ with

$$
\mathcal{A}^{2}=\frac{r}{g_{11}}=\frac{r}{1+\frac{1}{2}(2-g) e^{-2 k_{c}^{2} \sigma^{2}}},
$$

is already an exact solution of the full field dynamics Eq. (6.1).

In case of squares $1(n=2)$, the amplitude equations up to fifth order are given by

$$
\partial_{t} A_{1}=r A_{1}-\left(g_{11}\left|A_{1}\right|^{2}+g_{12}\left|A_{2}\right|^{2}\right) A_{1}+g_{211}\left|A_{1}\right|^{2}\left|A_{2}\right|^{2} A_{1}+g_{221}\left|A_{2}\right|^{4} A_{1},
$$

with

$$
g_{211}=\frac{1}{8}\left(g-1+(2-g) \frac{1}{2} e^{-2 \sigma^{2}}+(2-g) e^{-\sigma^{2}}\right)^{2},
$$

and $g_{221}=\frac{1}{2} g_{211}$. The third order amplitudes receive a correction Eq. (6.17) given by

$$
B_{1}=B_{2}=\frac{\left(g_{211}+g_{221}\right) A^{5}}{r-3 A^{2}\left(g_{11}+g_{12}\right)}=\frac{g_{5} r \sqrt{r}}{2 g_{3}^{2} \sqrt{g_{3}}}
$$

with $g_{3}=g_{11}+g_{12}$ and $g_{5}=g_{211}+g_{221}$. The stationary solutions of the amplitude equations Eq. (6.22) are

$$
\mathcal{A}_{1}=\mathcal{A}_{2}=\mathcal{A}=\sqrt{\frac{g_{3}}{2 g_{5}}-\frac{\sqrt{g_{5}^{2}-4 g_{5} r}}{2 g_{5}}} .
$$

A series expansion of this stationary solution leads to

$$
\mathcal{A}=\sqrt{r / g_{3}}+r^{3 / 2} \frac{g_{5}}{2 g_{3}^{2} \sqrt{g_{3}}}+r^{5 / 2} \frac{7 g_{5}^{2}}{8 g_{3}^{4} \sqrt{g_{3}}}+\ldots
$$

\footnotetext{
${ }^{1}$ The real part $\operatorname{Re}(z)$ of the $n=2$ ECP solution has a square layout.
} 
which agrees with Eq. (6.24) up to corrections of the order $r^{5 / 2}$. As $g_{5}$ is positive for all $g$ and $\sigma$ the fifth order corrections increase the amplitudes of the $n=2$ planforms.

\subsection{Phase relations}

In case of ECP solutions, for which $A_{i^{-}}=0$, the leading order amplitude equations are degenerate in the phases of the active modes. The fifth order amplitude equations Eq. (6.19) we derived for a general number of active modes are also degenerate in the phases in case of ECP solutions. In the following, we discuss how this degeneracy can be lifted at fifth order when special planform configurations are considered. We first show that triad resonances can lead to a partial lifting of the phase degeneracy. Moreover, we show that due to these resonances, solutions of the form $A_{i^{-}}=0$ no longer exist and thus fifth order corrections lead to small but non-zero opposite modes.

\subsubsection{Phase relations in essentially complex planforms}

First, consider phase relations in planforms for which $A_{i^{-}}=0$. As it turns out that the occurrence of phase equations is planform dependent we first consider the LDP solutions. If the number of active modes is a multiple of three $(n=3 l)$ triad resonances exist between the active modes i.e.

$$
\vec{k}_{i}-\vec{k}_{i+l}+\vec{k}_{i+2 l}=0, \quad l=1,2, \ldots \quad i=1, \ldots, l
$$

where the index $i$ is assumed to be cyclic $A_{i+n}=A_{i}$. These triad resonances can lead to additional contributions to the amplitude equations Eq. (6.19) which are then no more degenerate in their phases. The number of occurring phase equations can be reduced by symmetry. For instance, in systems with orientation shift symmetry the number of this terms is reduced. For the simplest case $l=1$ we get one triad resonance condition but no additional contribution to the amplitude equations. All three phases are free due to translational symmetry and orientation shift symmetry. Without orientation shift symmetry there is the possibility to have a phase equation already for $l=1$. For $l \geq 2$ we get the following additional contribution to the amplitude equations at fifth order

$$
\partial_{t} A_{i}=\cdots+\sum_{j \neq i}^{l} h_{i j} A_{j} \bar{A}_{j+l} A_{j+2 l} A_{i+l} \bar{A}_{i+2 l},
$$

where the dots stand for contributions already given in Eq. (6.19). Therefore we get $l-1$ additional terms in the amplitude equations. For instance, in the case of $n=6(l=2)$ the dynamics of the phase $\phi_{1}$ reads

$$
\mathcal{A}_{1} \partial_{t} \phi_{1}=h \mathcal{A}_{2} \mathcal{A}_{3} \mathcal{A}_{4} \mathcal{A}_{5} \mathcal{A}_{6} \sin \left(\phi_{2}+\phi_{3}+\phi_{6}-\phi_{5}-\phi_{4}-\phi_{1}\right)
$$


with $h>0$ and $h=2(18+\sqrt{3})(1-g)^{2}$ for $\sigma$ large. We can define a combined phase as

$$
\Phi=\phi_{1}+\phi_{4}+\phi_{5}-\phi_{2}-\phi_{3}-\phi_{6}
$$

Summing up all phase equations leads to

$$
\partial_{t} \Phi=-\alpha \sin (\Phi)
$$

The prefactor $\alpha$ depends on the amplitudes and the factor $h$. This phase equation can be derived from the potential $V[\Phi]=-\alpha \cos (\Phi)$. Stationary solutions are given by $\Phi^{*}=0$ and $\Phi^{*}=\pi$ and the sign of $\alpha$ determines which of these solutions are stable. As $h$ is positive for $0<g<1, \sigma>0$ the stationary phases are given by $\Phi^{*}=0$.

In the case of the $n=9(l=3)$ LDP solution the dynamics of the phase $\phi_{1}$ is given by

$$
\mathcal{A}_{1} \partial_{t} \phi_{1}=h \mathcal{A}_{2} \mathcal{A}_{4} \mathcal{A}_{8} \overline{\mathcal{A}}_{7} \overline{\mathcal{A}}_{2} \sin \Phi_{1}+\tilde{h} \mathcal{A}_{3} \mathcal{A}_{4} \mathcal{A}_{9} \overline{\mathcal{A}}_{7} \overline{\mathcal{A}}_{6} \sin \Phi_{2}
$$

with

$$
\begin{aligned}
& \Phi_{1}=\phi_{1}+\phi_{5}+\phi_{7}-\phi_{2}-\phi_{4}-\phi_{8} \\
& \Phi_{2}=\phi_{1}+\phi_{6}+\phi_{7}-\phi_{3}-\phi_{4}-\phi_{9} .
\end{aligned}
$$

For $\sigma$ large the prefactors are given by $h \approx 75.52(1-g)^{2}$ and $\tilde{h} \approx 27.38(1-g)^{2}$. Stationary solutions which are independent of the model parameters are given by $\Phi_{1}^{*}=0, \pi$ and $\Phi_{2}^{*}=0, \pi$ with $\Phi_{1}^{*}=\Phi_{2}^{*}=0$ the stable solutions. Note, if some of the wavevectors are flipped $\left(l_{j}=-1\right)$ the number of resonance relations can be reduced. For instance, in the case of $n=6$ the planform with $l=(1,1,1,-1,1,1)$ has no phase equations. In the case of $n=9$ the planform with $l=(1,1,1,1,1,-1,1,1,1)$ has only one phase equation for the modes $j=2,3,5,6,8,9$ while there are no phase equations for the modes $j=1,4,7$.

Besides these triad resonances there can be higher resonances. Additional relations between the wavevectors appear for $n=5 l, n=7 l, \ldots, l=1,2, \ldots$ These resonances, however, lead to phase equations in the amplitude equations beyond the fifth order. Again, for $l=1$ there are no phase equations due to orientation shift symmetry. But for $l \geq 2$ there are phase equations at ninth order in the amplitude expansion for $n=5 l$ or at thirteenth order for $n=7 l$. For instance, an $n=15$ planform at maximum has 4 phase equations at fifth order and additional two phase equations at ninth order. Finally, for $n=2^{l}, l=1,2, \ldots$ there are no triad or higher resonances and thus no phase equations at any order in the amplitude equations.

\subsubsection{The growth of opposite modes}

In Section 6.3 we have seen that if opposite modes are suppressed at leading order they receive no correction at fifth order for a general planform configuration. We will see in the following that in case of triad resonances the ECP solutions are modified because the opposite modes 

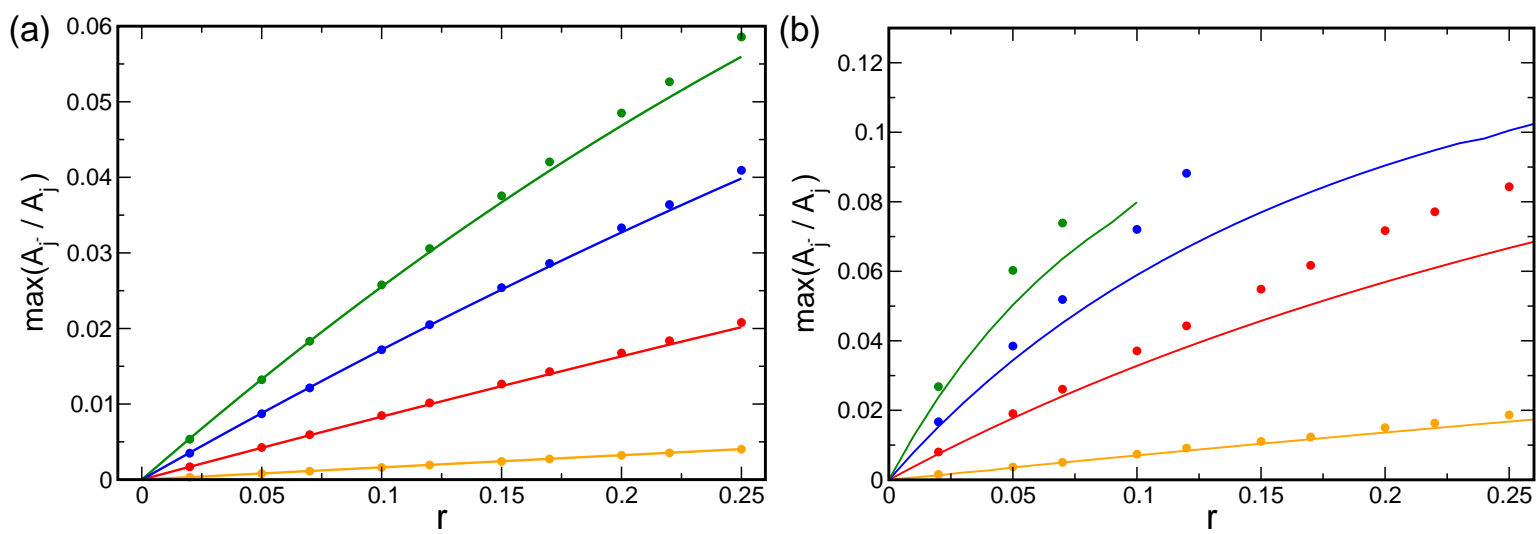

Figure 6.2.: Growth of opposite modes due to triad resonances. Ratio between modes $\mathcal{A}_{i}$ and opposite modes $\mathcal{A}_{i^{-}}$. (a) $n=3$, LDP planform. Solid lines: ratio obtained from solving amplitude equations, Eq. (6.19). Green: $g=0.7, \sigma=0.41 \Lambda$, blue: $g=0.8, \sigma=0.43 \Lambda$, red $g=0.9, \sigma=0.46 \Lambda$, orange: $g=0.98, \sigma=0.53 \Lambda$. (b) $n=6, \mathrm{LDP}$ planform. Green $g=0.7, \sigma=0.75 \Lambda$, blue: $g=0.8, \sigma=0.78 \Lambda$, red: $g=0.9, \sigma=0.85 \Lambda$, orange: $g=0.98, \sigma=1.0 \Lambda$. Dots: ratio obtained from solving the full field equations Eq. (6.1) numerically. Parameters: $\Gamma=22, T_{f}=10^{4}, 128 \times 128$ mesh.

are no longer suppressed in this case. At fifth order additional triad resonances appear for $n=3 l$. Compared to the case $A_{j^{-}}=0$ the phases are restricted already for $l=1$ as there are now $6 l$ phase degrees of freedom. These additional terms to Eq. (6.19) are given by

$$
\partial_{t} A_{i^{-}}=\cdots+h\left(A_{(i+1)^{-}}^{2} \bar{A}_{(i+2)^{-}}^{2} A_{i}+A_{(i+2)}^{2} \bar{A}_{(i+1)}^{2} A_{i}\right)+\tilde{h} A_{(i+1)^{-}} \bar{A}_{(i+1)} A_{(i+2)} \bar{A}_{(i+2)^{-}} A_{i}
$$

with $A_{i+n}=A_{i}$. Due to the second term solutions with $A_{i^{-}}=0$ do not exist. The inclusion of opposite modes restricts the phases dynamics already at leading order, see Eq. (6.13). Again the occurrence of such terms depends on the planform configuration. The $n=3$ HDP solution has no such terms and thus the opposite modes are still suppressed at fifth order. The ratio between modes $A_{j}$ and opposite modes $A_{j^{-}}$for an $n=3$ and $n=6$ LDP solution is shown in Fig. 6.2. With varying bifurcation parameter the ratio changes as $A_{j^{-}} / A_{j} \propto \frac{r^{3 / 2}}{r^{1 / 2}+r^{3 / 2}}=r /(1+r)$. Both, the modes $A_{j}$ and the opposite modes $A_{j^{-}}$are modulated in their amplitudes. We therefore calculate the ratio $A_{j^{-}} / A_{j}$ for each pair $j$ of mode-opposite mode configuration and plotted their maximum. Modulations within each set $\left\{A_{j}\right\}$ and $\left\{A_{j^{-}}\right\}$occur due to higher order corrections and the spatial discretization but are weak for the chosen parameters. The ratios obtained from solving the full field dynamics are in good agreement with those obtained from amplitude equations. For large $r$ or small $g$ the ratios of the full field dynamics are slightly higher.

\subsubsection{Phase relations in numerical simulations}

To confirm the existence of phase relations found in the higher order amplitude equations and to check whether there are additional phase relations not predicted by the amplitude equations we solved the full field dynamics Eq. (6.1) numerically. The dynamics of phases is 
determined in late stages of development and we therefore have to integrate over long times. For such simulations the time evolution of phases of an $n=6$ planform is shown in Fig. 6.3(a). Using a LDP solution with a random phase configuration as initial condition we observe that all the phases adjust at the same time, $T \approx 10^{2}$. At the final time $T_{f}=T^{*}=10^{5}$ we shift one phase by a constant amount $\phi_{i}=\phi_{i}+1.4$. The dynamics then evolves again until $T=T^{*}+10^{5}$. The perturbation at $T=T^{*}$ leads to a readjustment of the phases indicating that their stationary values are indeed not arbitrary. The stationary phases are $\Phi^{*}=0$, see Fig. 6.3(b), as predicted by amplitude equations. The time evolution of the phases for an $n=9 \mathrm{LDP}$ solution is shown in Fig. 6.3(c). In this case there are two terms in the phase dynamics leading to the two stationary phases $\Phi_{1}=\Phi_{2}=0$, see Eq. (6.33). The phases all adjust at $T \approx 10^{4}$. Note, that similar results are obtained for different shifts of the phase at $T=T^{*}$.

As mentioned before, not all planform configurations lead to phase equations. For instance, the $n=6, l=(1,1,-1,1,1,1)$ planform is expected not to lead to phase restrictions. Indeed, in numerical simulations the initially random phases are not altered during time evolution, i.e. there is a degeneracy of the phases, see Fig. 6.3(e). We next studied the time evolution of the phases for an $n=8$ planform. Here, we do not expect any phase relations at any order in the amplitude equations. The time evolution of their phases is shown in Fig. 6.3(f). When studying phase equations we have to ensure a high spatial resolution of the critical circle. A reason for this can be additional phase relations that appear due to the spatial discretization of the pattern. Indeed the phase dynamics changes if we increase the spatial resolution of the system by increasing the aspect ratio and system size. In Fig. 6.3(f) the initial phases remain unchanged and a perturbation of one phase does not lead to a later adjustment of the phases. To summarize, even considering higher order corrections to the amplitude equations, the phase degeneracy is only partly lifted. Due to translation and orientation shift symmetry three modes are left undetermined in all cases. From the remaining phases some of them are fixed by amplitude equations at fifth or even higher order. Phase restrictions due to the triad resonance are thus rare and most of the phases remain undetermined. The occurrence of phase relations is furthermore planform dependent where their number is maximal for LDP solutions. 
(a)

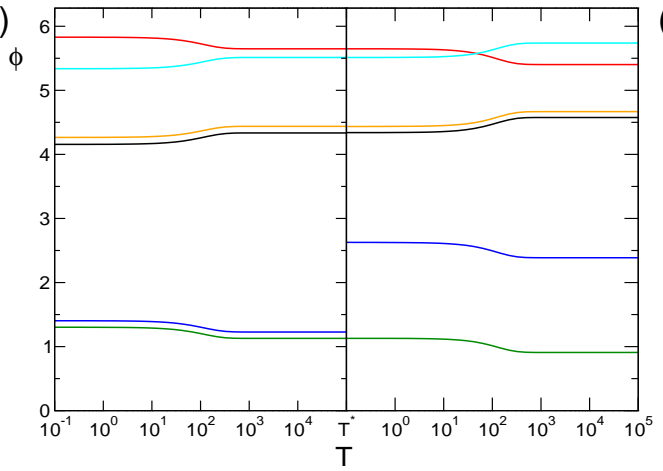

(c)

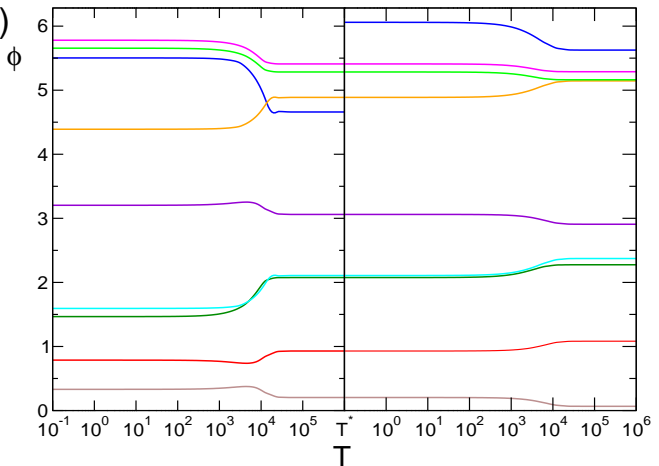

(e)

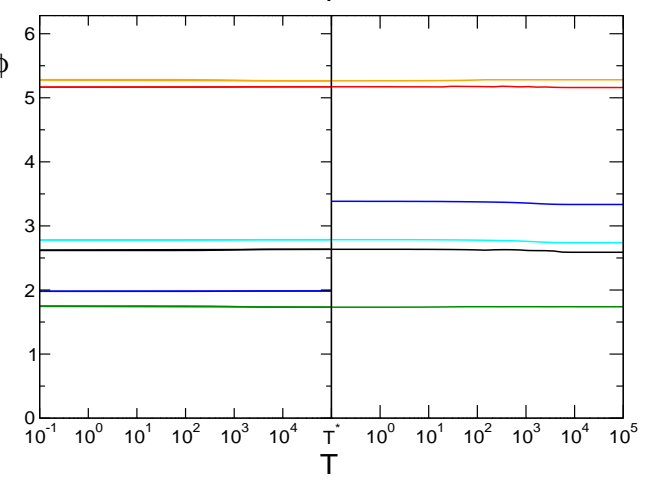

(b)

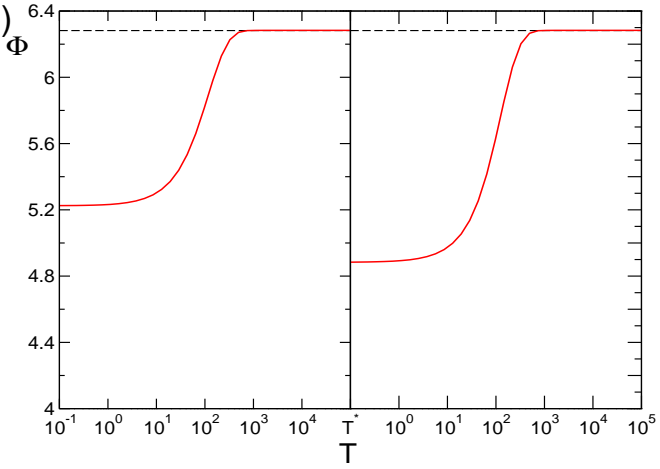

(d)

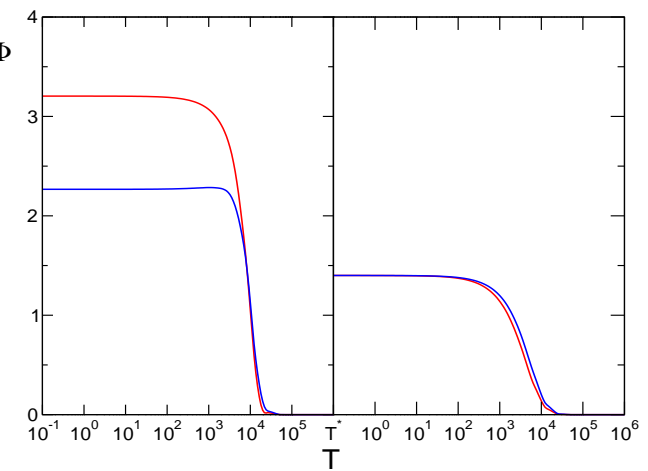

(f)

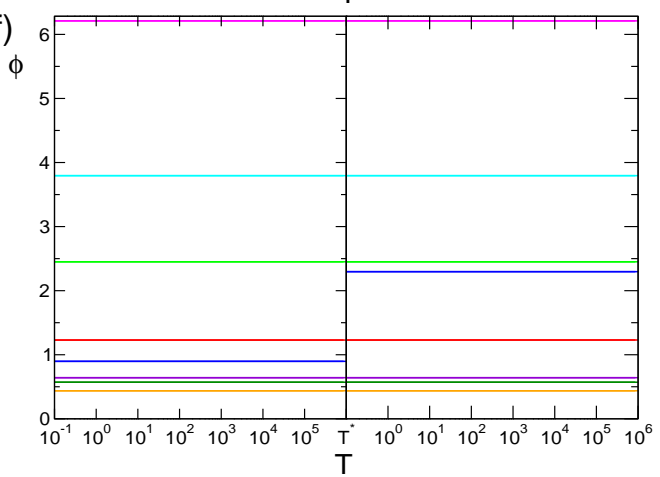

Figure 6.3.: Time evolution of the phases. The patterns evolves until stationary phases are reached. At this time $\left(T=T^{*}\right)$ one phase (blue line) is shifted by a constant amount and the dynamics evolves again. (a) $n=6$, LDP solution. Parameters: $T^{*}=10^{5}, g=0.9, \sigma=0.92 \Lambda, r=0.1, \Gamma=22,128 \times 128$ mesh. (b) $\Phi=\phi_{1}+\phi_{4}+\phi_{5}-\phi_{2}-\phi_{3}-\phi_{6}$. (c) $n=9$, LDP solution. Parameters: $T^{*}=10^{6}, r=0.1, g=0.98, \sigma=1.61 \Lambda, \Gamma=100,512 \times 512$ mesh. (d) $\Phi_{1}, \Phi_{2}$, see Eq. (6.33). (e) $n=6, l=(1,1,-1,1,1,1)$ planform. Parameters: $T^{*}=10^{5}, g=0.9, \sigma=0.92 \Lambda$, $r=0.1, \Gamma=22,128 \times 128$ mesh. (f) $n=8$, LDP solution. Parameters:

$T^{*}=10^{6}, r=0.1, g=0.98, \sigma=1.43 \Lambda, \Gamma=80,512 \times 512$ mesh. 


\subsection{Stationary amplitudes}

How do fifth order corrections affect the stationary amplitudes of ECP solutions? In leading order amplitude equations permutation symmetry guarantees uniform amplitudes which can be calculated analytically. Beyond the simple case $n=2$ the stationary amplitudes of ECP solutions at fifth order are in general no longer uniform. We thus calculate numerically the stationary solutions of the higher order amplitude equations Eq. (6.19) with increasing distance from threshold. Figure 6.4 shows the ratio of the fifth and third order amplitudes for different numbers of active modes $n$. As the third order amplitudes $A^{(3)} \propto \sqrt{r}$ and fifth order corrections scale as $r^{3 / 2}$ the ratio increases linearly with $r$. In general, higher order corrections shift most of the amplitudes of LDP solutions towards higher values. We observe that the fifth order corrections are smaller in case of the HDP compared to the LDP solutions. In symmetric HDP ( $n$ odd) configurations the stationary amplitudes are still uniform due to symmetry, see black dashed lines in Fig. 6.4(a,c). In the remaining cases there are modulations of the amplitudes. For instance, in case of $n=3$ the mode at $\alpha=\pi / 3$ is larger compared to the remaining two modes. This convex shape of amplitude modulations is observed also for larger $n$. But note, although the convex shape is robust, the exact form of amplitude modulations also depends on the parameter $g$. With an increasing number of active modes the modulations in the amplitudes increase. The observed modulations of the stationary amplitudes result in a slightly more anisotropic OP layout. We discuss in the following section how this impacts the resulting pinwheel densities. 

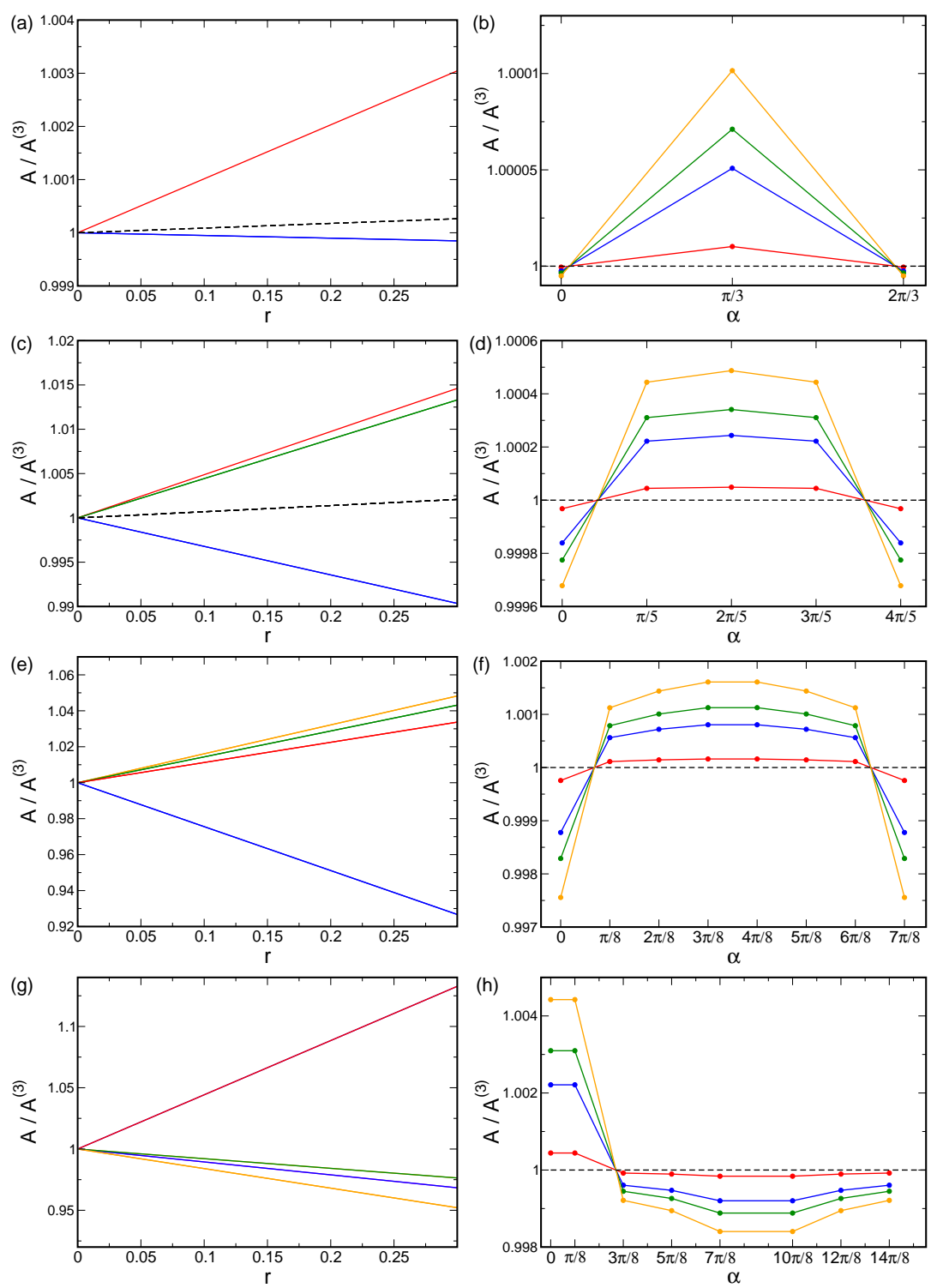

Figure 6.4.: Stationary amplitudes at fifth order. Ratio of fifth order amplitudes $A$ Eq. (6.19) and third order amplitudes $A^{(3)}$ Eq. (6.13). (a,b) $n=3, \sigma=0.5 \Lambda$, colored lines: LDP, black dashed line: HDP. (c,d) $n=5, \sigma=0.8 \Lambda$, colored lines: LDP, black dashed line: HDP. (e,f) $n=8 \mathrm{LDP}, \sigma=1.23 \Lambda$. (g,h) $n=8 \mathrm{HDP}, \sigma=1.23 \Lambda$. Right panel:

Modulations of the amplitudes as a function of the angle $\alpha$ on the critical circle. Red: $r=0.001$, blue: $r=0.01$, green: $r=0.05$, orange: $r=0.1$. In all conditions $g=0.9$. 


\subsection{Pinwheel densities - Near and far from threshold}

In this section we discuss the pinwheel densities of ECP solutions near and far from threshold. First, we discuss pinwheel densities close to threshold obtained from solutions of the leading order amplitude equations. Next, we compare these densities with those obtained from solving the full field dynamics numerically. We observe that, close to threshold, the obtained densities agree with those of the amplitude equations whereas they deviate far from threshold. Finally, we want to answer the question of whether higher order corrections which influence the pinwheel densities of ECP solutions can explain this deviation far from threshold.

The stationary solutions to the amplitude equations derived from Eq. (6.1) are degenerate in their phases. To calculate the pinwheel density for each planform solution we therefore need to average an ensemble of realizations with random phase configurations. Different realizations were collected $(\Gamma=22$, mesh $=1024 \times 1024)$ until sufficient precision of average pinwheel densities was reached, measured by the standard error $S D / \sqrt{N}$ where $S D$ is the standard deviation and $N$ the number of realizations. For planforms with $n \leq 14$ the required precision is $S D / \sqrt{N}<0.03$ while for $n \geq 15$ the precision is $S D / \sqrt{N}<0.01$. Different ECP solutions vary substantially in their pinwheel density. This variation is illustrated in Fig. 6.5(a). For intermediate and large $n$, pinwheel densities fall into a band which for large $n$ is in the range between $\rho=1.36$ and $\rho=3.5$. Whereas individual realizations of planforms show a large variety in the pinwheel density, the ensemble average over all planforms with a given number of active modes shows a robust selection of distinct pinwheel densities. The average over the ensemble of planforms with a given $n$ is obtained by randomly choosing $\phi_{j}$ and $l_{j}= \pm 1$. Fig. 6.5(b) shows the average density for $3 \leq n \leq 17$. With increasing $n$ the average density approaches $\rho=\pi$ from below. It has been shown that in the limit $n \rightarrow \infty$ the average pinwheel density is $\rho=\pi$, and the variation in the density decreases such that almost every single realization has a pinwheel density of $\rho=\pi$ [16]. Taking a closer look at the distribution of pinwheel densities we observe that the pinwheel density for a given planform can be classified to some extent. The lowest pinwheel density is always given by the LDP. Flipping Fourier modes in general increases the pinwheel density. To classify the different planforms for a given number of active modes $n$ we define the planform anisotropy

$$
\vec{\xi}=\frac{\pi}{2 k_{c} n} \sum_{j}^{n} l_{j} \vec{k}_{j}
$$

For large $n$ the modulus $\xi=|\vec{\xi}|$ is bounded by $0 \leq \xi \leq 1$. How the pinwheel density varies with planform anisotropy is shown in Fig. 6.5(c). With increasing anisotropy the pinwheel density decreases. In the large $n$ limit the pinwheel density as a function of the anisotropy can be calculated analytically [49] and is given by

$$
\rho(\xi)=\sqrt{\pi^{2}-8 \xi^{2}}
$$



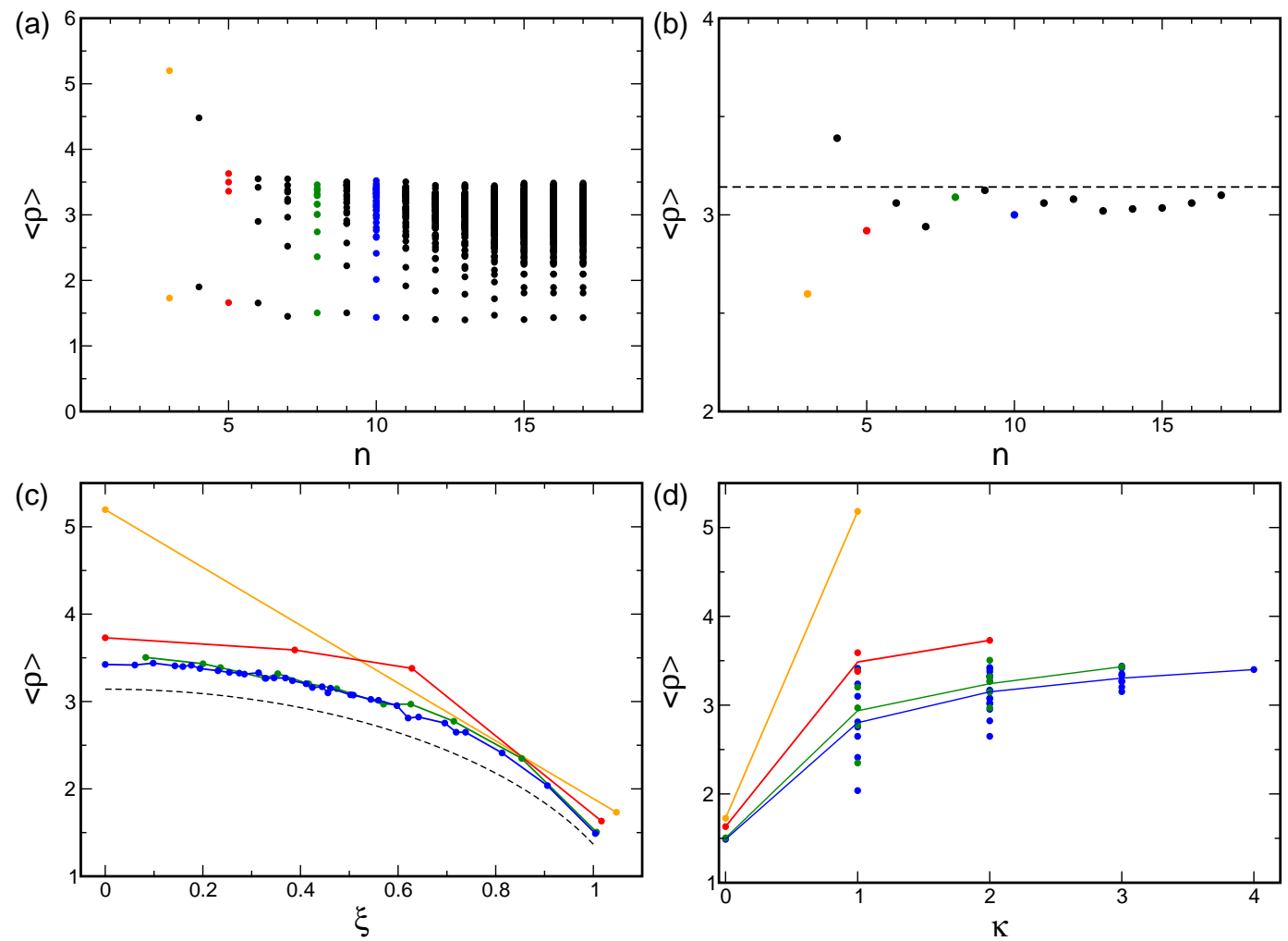

Figure 6.5.: Pinwheel densities near threshold. (a) Pinwheel density for ECP solutions. The points mark the numerically determined densities. Each value represents an average over the ensemble of phases $\phi_{j}$. (b) Pinwheel densities averaged over different realizations with random $l_{j}$, dashed line: $\rho=\pi$. (c) Pinwheel density as a function of the planform anisotropy $\xi$, dashed line: pinwheel density in the large $n$ limit, see Eq. (6.36). Orange: $n=3$, red: $n=5$, green: $n=8$, blue: $n=10$. (d) Pinwheel density as a function of the number of flipped mode clusters $\kappa$. Solid line: average pinwheel density for fixed $\kappa$.

We further define a cluster of flipped modes as those modes $\vec{k}_{u}, \vec{k}_{u+1}, \ldots, \vec{k}_{v}, v \geq u$ for which $l_{u}=l_{u+1}=\cdots=l_{v}=-1$ and $l_{u-1}=l_{v+1}=1$. The number of such flipped mode clusters is denoted by $\kappa$. The pinwheel densities as a function of $\kappa$ are shown in Fig. 6.5(d). For a given number of flipped mode clusters the corresponding planforms still vary in their pinwheel density, but the mean pinwheel density of those planforms increases with $\kappa$, see Fig. 6.5(d). How are the pinwheel densities modified for substantial distance from threshold? To answer this question we calculated the pinwheel densities for transient as well as for final states of the full field dynamics Eq. (6.1) and increasing distance from threshold $r$, see Fig. 6.6. We observe that for intermediate times $(T \approx 300)$ the average pinwheel density is largely insensitive to the model parameters $r$ and $\sigma$. Even far from threshold the average pinwheel density is larger than $\langle\rho\rangle=\pi$ and thus similar to densities obtained in the visual cortex. In contrast, at the final time $\left(T=10^{5}\right)$ average pinwheel densities are very sensitive to the model parameters. For small $r$, the average pinwheel density is close to $\rho=3$. With increasing $r$, however, the average pinwheel density is decreasing. Note, for intermediate $n$ the average pinwheel density of the ECP solutions at leading order is smaller than $\pi$, see Fig. 6.5(b). For $r \approx 0.05$ 

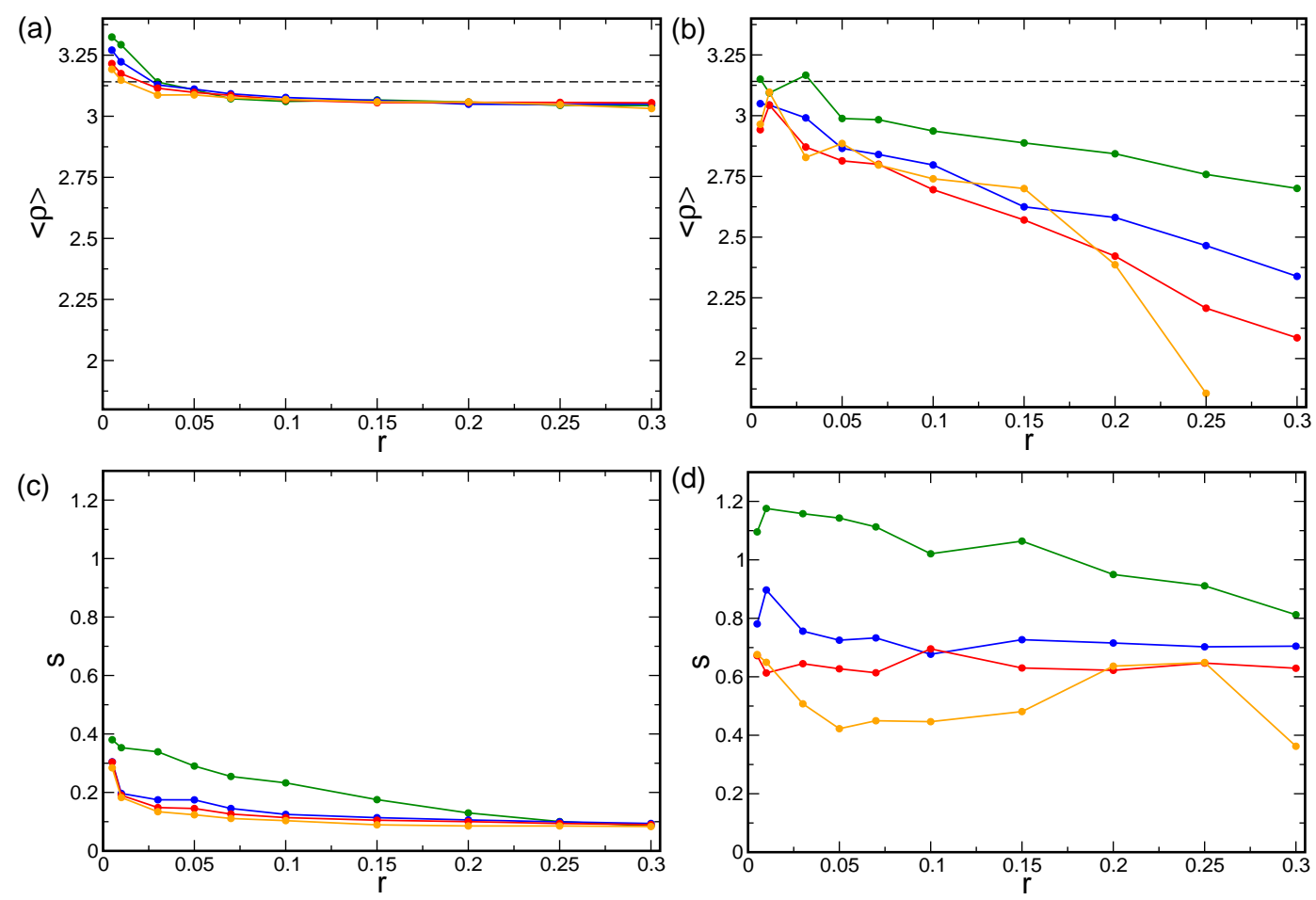

Figure 6.6.: Pinwheel densities for the full field dynamics Eq. (6.1). (a,b) Average pinwheel densities. The numerical solutions are obtained at (a) $T=300$, (b) $T=10^{5}$, starting from an identical set of $N=50$ initial conditions. Green: $\sigma=0.9 \Lambda$, blue: $\sigma=1.43 \Lambda$, red: $\sigma=1.7 \Lambda$, orange: $\sigma=2.0 \Lambda$. Dashed line: predicted value $\langle\rho\rangle=\pi$ from the leading order amplitude equations and large $n$. $(\mathbf{c}, \mathbf{d})$ Standard deviation of the pinwheel densities. Parameters: $g=0.98, \Gamma=22,128 \times 128$ mesh.

all observed pinwheel densities are lower than $\rho=3$. For larger values of the interaction range $\sigma$ i.e. for more active modes this deviation becomes stronger. We further observe that the number of realized LDP solutions increases with increasing $r$. In the following we want to reveal the influence of fifth order corrections on the pinwheel density of ECP solutions. Fifth order corrections can change their pinwheel densities for several reasons. For each planform, potential modulations of the corresponding stationary amplitudes will lead to a change in the pinwheel density. In addition, contributions from modes off the critical circle can alter the pinwheel density. Moreover, as we discuss in Section 6.4 phase restrictions which can occur at higher order could also alter the pinwheel densities. Pinwheel densities at higher order are calculated from the field $z=\sqrt{r} z_{1}+r^{3 / 2} z_{3}$, see Eq. (6.2), where the third and fifth order fields are given by Eq. (6.4) and Eq. (6.14), respectively. The pinwheel densities for stationary patterns at fifth order is shown in Fig. 6.7. The third order pinwheel densities where the stationary amplitudes are uniform correspond to $r=0$. We plot the pinwheel densities for two parameter values, $g=0.98$ with a weak influence of the fifth order corrections and a lower value of $g$ at which fifth order corrections are more pronounced. The amount of pinwheel density reduction is planform dependent. For the symmetric planform configurations (the HDP in case of $n=5$ and the configuration $l=(1,1,-1,-1,1,1)$ in case 

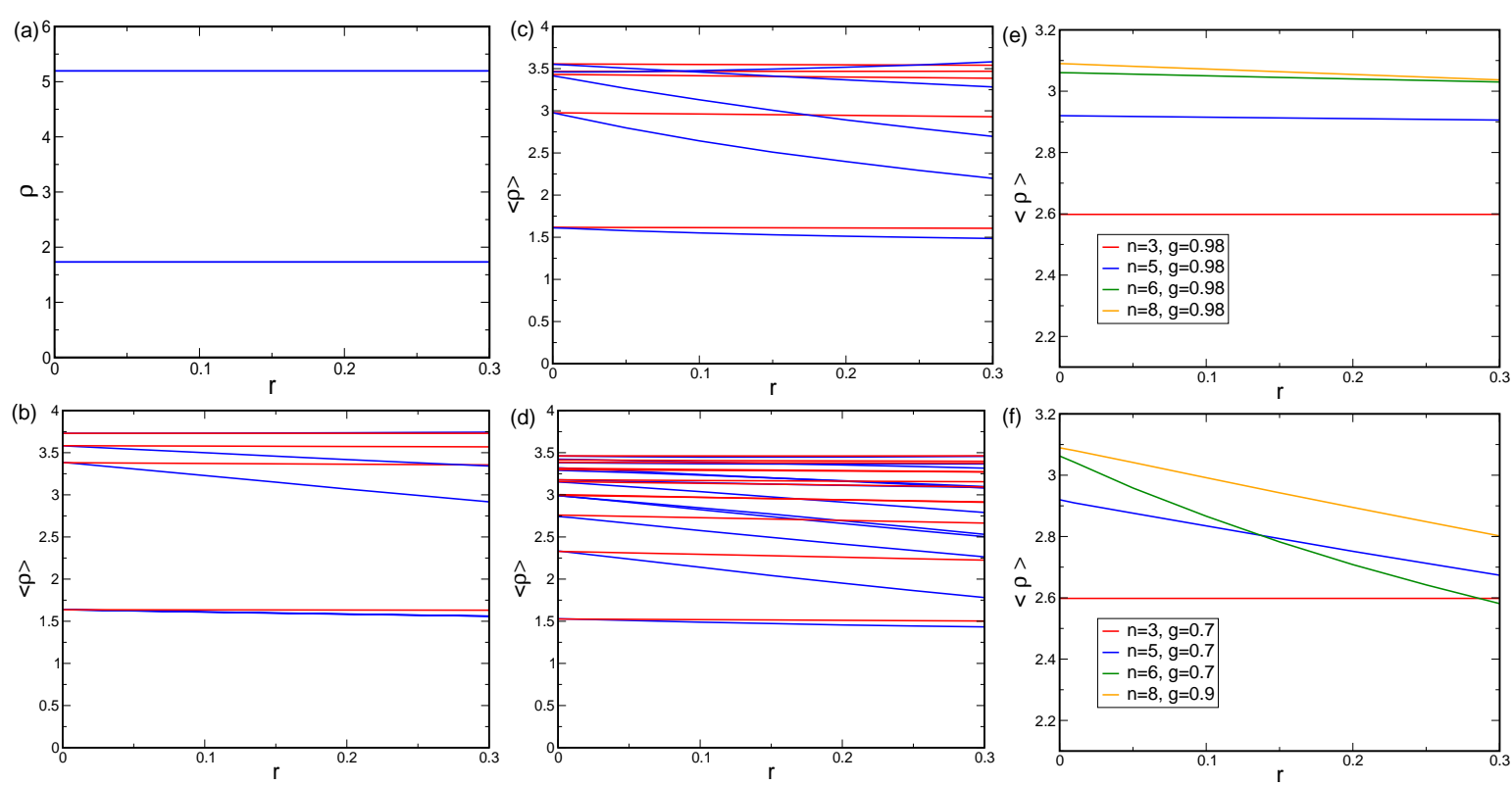

Figure 6.7.: Pinwheel densities at fifth order. (a) $n=3$ planforms $g=0.7, \sigma=0.5 \Lambda$, (b) $n=5$ planforms, $g=0.7, \sigma=0.7 \Lambda$ (blue), $g=0.98, \sigma=0.9 \Lambda$ (red), (c) $n=6$ planforms, $g=0.7, \sigma=0.75 \Lambda$ (blue), $g=0.98, \sigma=\Lambda$ (red), (d) $n=8$ planforms, $g=0.9, \sigma=1.23 \Lambda$ (blue), $g=0.98, \sigma=1.4 \Lambda$ (red). (e,f) Average over random planform realizations. Pinwheel densities at $r=0$ correspond to the third order result.

of $n=6$ ) pinwheel densities are almost unchanged. Stationary amplitudes are still uniform and pinwheel densities in such planforms can be changed only by contributions from modes off the critical circle. In all remaining planforms the modulations in the amplitudes are such that the pinwheel densities are decreased with increasing $r$. We calculate the average over random planform realizations i.e. realizations with randomly chosen $l_{j}$, see Fig. 6.7(e,f). The average pinwheel density of the $n=5$ planforms decreases from $\rho \approx 2.92$ to $\rho \approx 2.91$ for $g=0.98$ and to $\rho \approx 2.67$ for $g=0.7$. The average pinwheel density of the $n=6$ planforms decreases from $\rho \approx 3.06$ to $\rho \approx 3.03$ for $g=0.98$ and to $\rho \approx 2.58$ for $g=0.7$. Finally, the average pinwheel density of the $n=8$ planforms decreases from $\rho \approx 3.09$ to $\rho \approx 3.04$ for $g=0.98$ and to $\rho \approx 2.8$ for $g=0.9$. If we compare the reduction in the average pinwheel densities for $g=0.98$ with those obtained from the full field dynamics, see Fig. 6.6, we observe that higher order corrections to the ECP solutions alone cannot fully explain the observed reduction in the pinwheel density. As we will see in the following, higher order corrections lift the energetic degeneracy of planform solutions which exists at leading order. Thus at fifth order there is a selection of certain planform solutions which can also change the pinwheel density. We will discuss this pattern selection in the next section.

\subsection{Lifting the planform degeneracy}

We have seen in the previous section that higher order corrections to ECP solutions are not sufficient to explain the amount of decrease in the pinwheel densities. We therefore study how 
higher order corrections lift the degeneracy of the ECP solutions and whether an energetic selection of certain planforms can lead to a more pronounced decrease in the pinwheel density. The amplitude equations Eq. (6.19) can be derived from a potential which to fifth order takes the form

$$
\begin{aligned}
V[A]= & -r \sum_{i}^{n}\left|A_{i}\right|^{2}+\frac{1}{2} \sum_{i, j}^{n} g_{i j}\left|A_{i}\right|^{2}\left|A_{j}\right|^{2}+\frac{1}{2} \sum_{i, j}^{n} f_{i j} A_{j} A_{j}-\bar{A}_{i} \bar{A}_{i^{-}} \\
& +\frac{1}{3} \sum_{i, j, k}^{n} g_{i j k}\left|A_{i}\right|^{2}\left|A_{j}\right|^{2}\left|A_{k}\right|^{2} \\
& +\frac{1}{3} \sum_{i, j, k}^{n} f_{i j k}\left|A_{k}\right|^{2} A_{j} A_{j^{-}} \bar{A}_{i^{-}} \bar{A}_{i}+\frac{1}{3} \sum_{i, j, k}^{n} \widetilde{f}_{i j k} \bar{A}_{k} \bar{A}_{k^{-}} A_{j} A_{j^{-}} A_{i} \bar{A}_{i} .
\end{aligned}
$$

Note, that additional contributions to the potential can occur in case of triad resonances, see Section 6.4. The potential is real valued because of the symmetries $f_{i j}=f_{j i}$ and $f_{i j k}=f_{j i k}$. At third order the potential $V_{3}$ is degenerate in the different ECP solutions for a given number of active modes [49,50], see also Fig. 5.1(b). Corrections beyond the third order lead to a planform dependence of the potential. We already revealed one origin for the planform dependence. Stationary amplitudes receive a planform dependent correction at fifth order and are in general no longer uniform. Moreover, the coupling coefficients become planform dependent at fifth order. Since fifth order corrections to the potential occur with a negative sign, these corrections lead in general to a decrease of the potential for all planforms. We calculate the potential of all ECP solutions for a given number of active modes $n$. The ratio of the fifth $V$ and third $V_{3}$ order potential is shown in Fig. 6.8 with increasing distance from threshold. As $V_{3} \propto r^{2}$ and $V \propto r^{2}+r^{3}$ the ratio increases linearly with $r$. We observe that for all $n$ the LDP solution ( $\kappa=0$ planforms, red lines) is energetically selected while the HDP solution (maximal $\kappa$, blue lines) has the largest energy. If the parameter $g$ is not too small (here $g=0.9$ ), the potential at fifth order can be clustered according to the number of flipped modes clusters $\kappa$. The origin of this clustering is discussed in Section 6.8. The potential difference increases with an increasing number of active modes $n$ but is still small at $g=0.9$. This indicates that amplitude modulations and energetic selection of LDP solutions cannot be the only reason for pinwheel density suppression far from threshold. We expect that the selection of LDP solutions also substantially changes the basins of attraction.

Fifth order corrections also change the phase diagram of the leading order ECP solutions, see Fig. 5.1(b). Figure 6.9 shows the influence of fifth order corrections on the phase diagram. Shown are the regions where an $n$ mode ECP has minimal energy. Whereas in the leading order there is a degeneracy of planform solutions at fifth order we have to consider all possible planforms for a given $n$. We thus calculate the potential Eq. (6.37) for all 93 ECP solutions which exist up to $n=10$. We observe that with increasing $r, \sigma$ (or $n$ ) and decreasing $g$ the influence of the fifth order corrections increases. In general, we observe a shift of all borders towards smaller $\sigma$ values. Moreover, this shift depends on $g$ and increases with decreasing $g$. Thus for $g$ near 1 the phase diagram resembles the phase diagram obtained at leading 

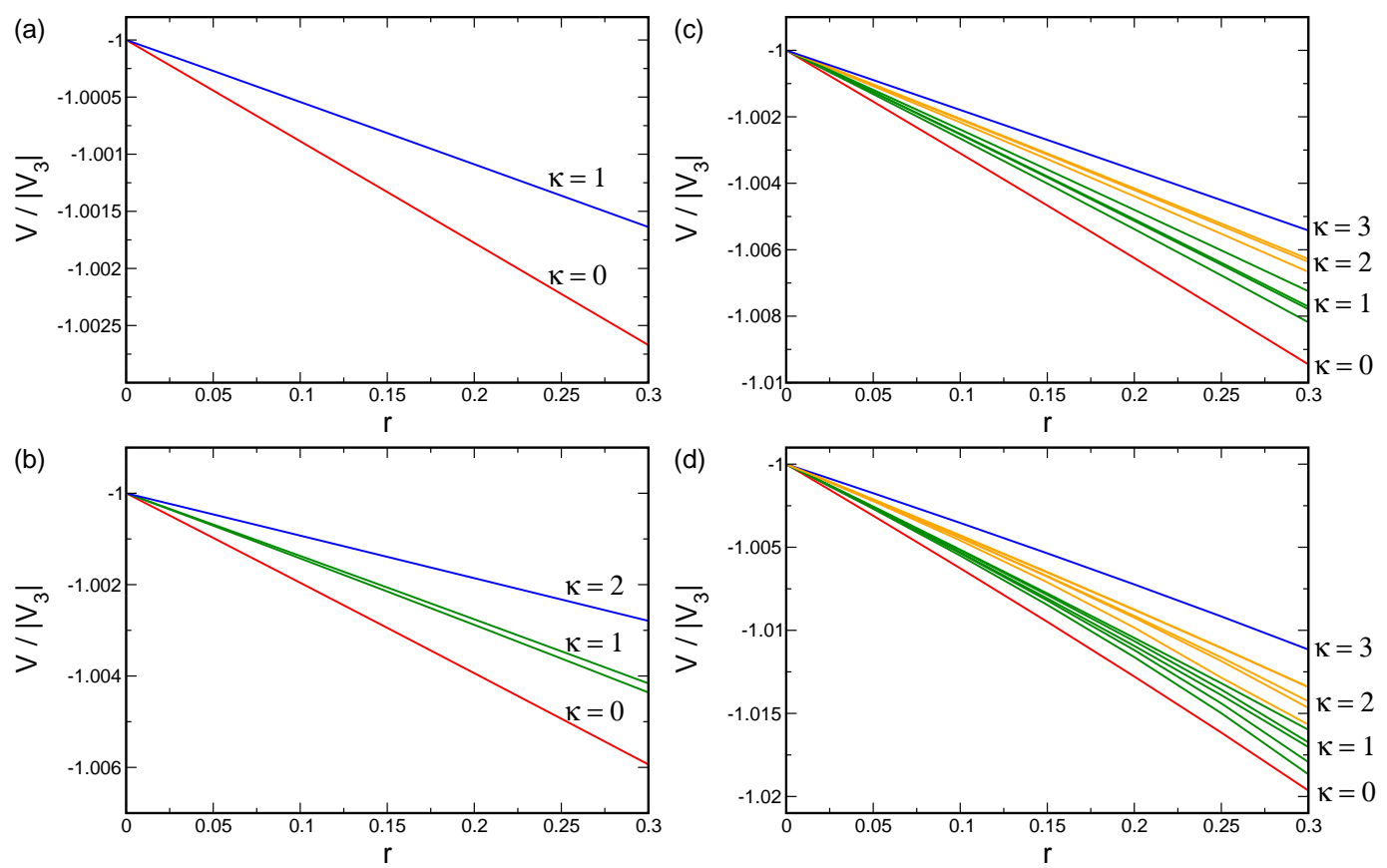

Figure 6.8.: Potential Eq. (6.37) at fifth order. (a) $n=4, \sigma=0.6 \Lambda$, (b) $n=5, \sigma=0.8 \Lambda$, (c) $n=7, \sigma=0.95 \Lambda$, (d) $n=8, \sigma=1.3 \Lambda$. In all conditions: $g=0.9$.

order. Compared to other planform solutions, the $n=3, n=6$, and $n=9$ ECP solutions (red) occupy a larger regime in which they are energetically preferred. We can explain this by the occurrence of phase relations at fifth order for these planforms which reduce the energy additionally, see Section 6.4. As already indicated by Fig. 6.8 we observe that in the whole parameter region the LDP solution has always the lowest energy. Remarkably, there are regions in the parameter space in which the potential for certain planform solutions is not bounded from below (gray regions). Typically this happens for small $g$, large $\sigma$, and large $r$. When the potential is unbounded the corresponding dynamics is unstable. We will discuss this instability of the dynamics in Section 6.9.3. Due to this instability the phase diagram is only valid either in a restricted $(g, \sigma / \Lambda)$ parameter space or close to threshold.

\subsection{The influence of non-resonant modes}

The aim of this section is twofold. First, we want to disentangle the different contributions to the fifth order potential and study how they lead to the observed planform dependence of the potential. Second, we want to discuss how fifth order corrections depend on the model parameters $g$ and $\sigma$. This will lead to a proposed rescaled model in which the distance to threshold becomes parameter dependent such that fifth order corrections can be neglected throughout the phase diagram.

We start with investigating the influence of the model parameter $g$ on fifth order corrections of the potential which are of the form $\sum_{i, j, k}\left|A_{i}\right|^{2}\left|A_{j}\right|^{2}\left|A_{k}\right|^{2} g_{i j k}$. Stationary amplitudes at third order scale as $\mathcal{A} \approx \sqrt{r /(1+g(n-1))}$ and fifth order corrections in general increase these 

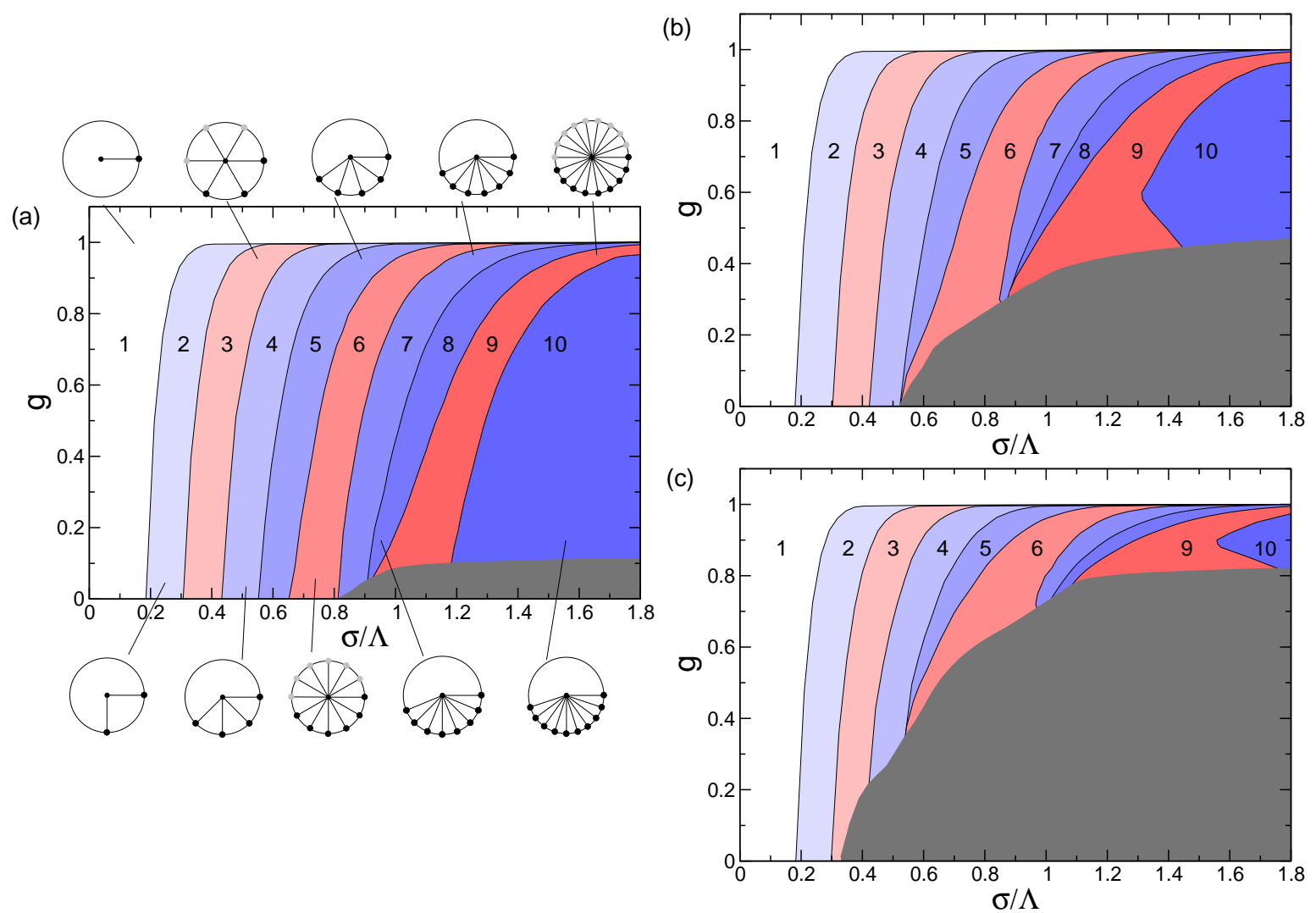

Figure 6.9.: Phase diagram with the inclusion of higher order corrections. Shown are the regions where a certain planform has the minimal energy Eq. (6.37). (a) $r=0.001$, (b) $r=0.01$ (c) $r=0.1$. Colored regions: LDP has minimal energy, white region: stripes have minimal energy, gray region: potential is not bounded from below.

amplitudes. In the following we assume $A_{j^{-}} \approx 0$ and small amplitude modulations $A_{j} \approx \mathcal{A}$. The sum over all fifth order coupling coefficients scales for large $\sigma$ as $S=\sum_{i, j, k} g_{i j k} \propto(g-1)^{2}$. Therefore fifth order contributions increase at least quadratically with $g$, and in the limit $g \rightarrow 1$ these corrections vanish. The relative energy change in the potential due to fifth order corrections is shown in Fig. 6.10(a) for different values of $g$. We observe that for $g$ close to one, the potential is clustered according to the number of flipped modes clusters $\kappa$. This clustering results from the clustering of the quantity $S$, see Fig. 6.10(c). For smaller values of $g$, however, we observe deviations of the clustering which result from modulations in the stationary amplitudes.

With an increasing number of active modes or with an increasing $\sigma$ the influence of fifth order corrections increase. How can an increase in $n$ be captured by moving closer to threshold i.e. reducing $r$ ? To answer this question we consider the distribution of modes off the critical circle which contribute to the fifth order coupling coefficients. The coupling terms in Eq. (6.19), see also Appendix A.5, are composed of contributions from the nonlinearity and from the inverse linear operator $\left(\hat{L}^{0}\right)^{-1}$ acting on the modes off the critical circle. We want to reveal the influence of these modes on the energy levels for different ECP solutions. The configuration non-resonant modes for three $n=5$ ECP solutions is illustrated in Fig. 6.11, 
(a)

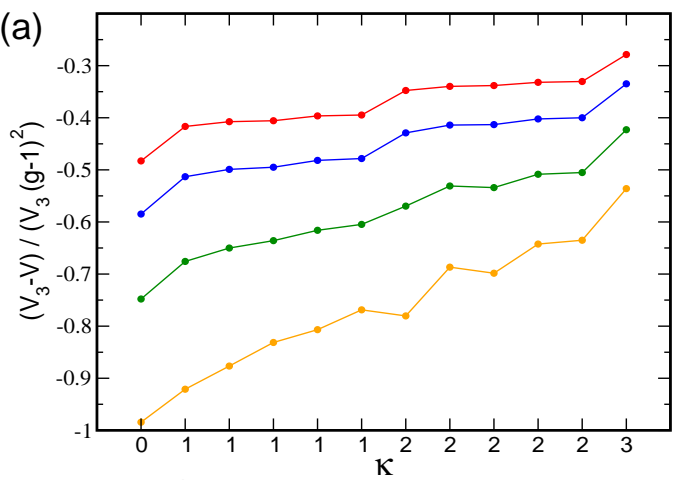

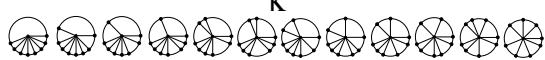

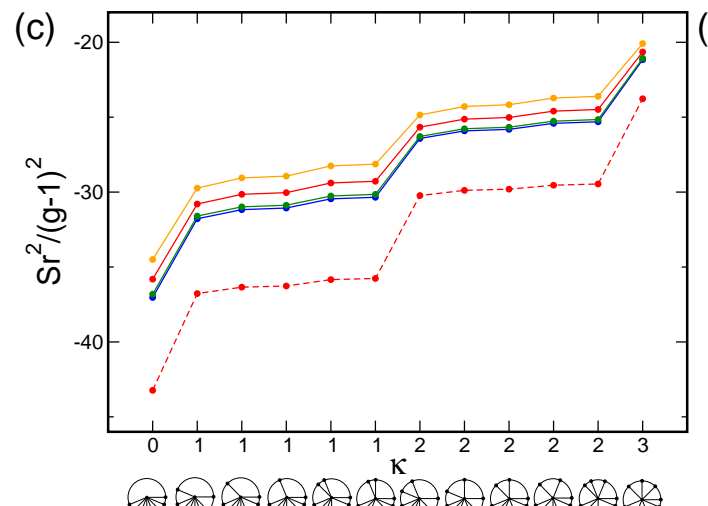

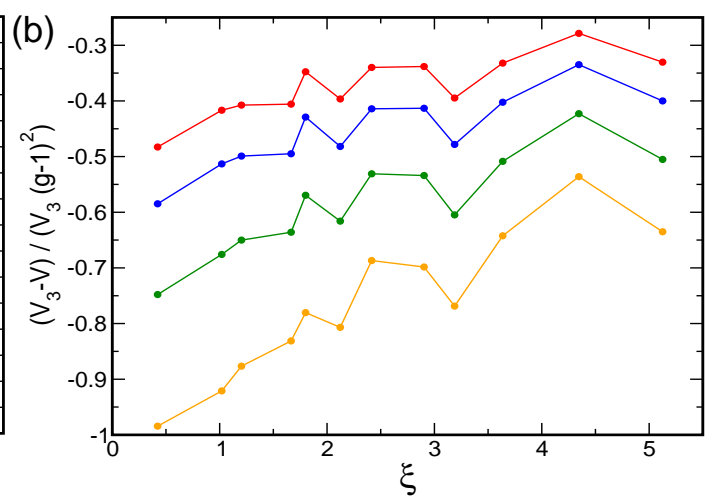

(d)

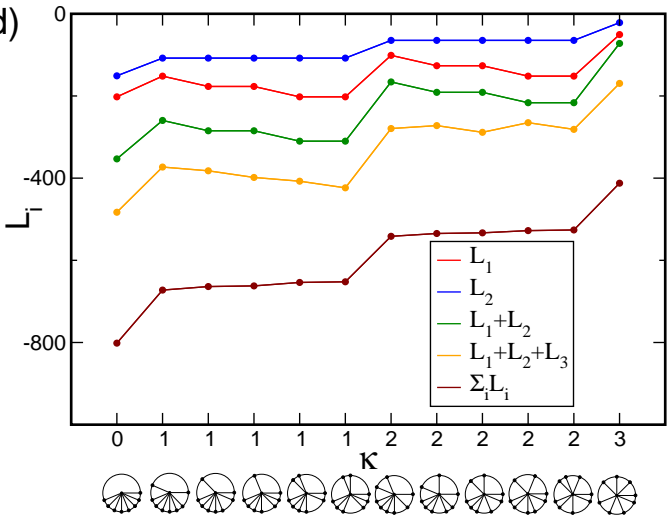

Figure 6.10.: Lifting of planform degeneracy for all $\mathbf{n}=\mathbf{8}$ planforms. (a) Relative energy change due to fifth order corrections as a function of the number of flipped modes, (b) as a function of the planform anisotropy. Red: $g=0.98, \sigma=1.4 \Lambda$, blue:

$g=0.9, \sigma=1.23 \Lambda$, green: $g=0.8, \sigma=1.14 \Lambda$, orange: $g=0.7, \sigma=1.04 \Lambda$, in all cases

$r=0.1$. (c) Sum over fifth order coupling coefficients. Red: $g=0.98, \sigma=1.4 \Lambda$, red dashed:

$g=0.98, \sigma=2.4 \Lambda$ blue: $g=0.9, \sigma=1.23 \Lambda$, green: $g=0.8, \sigma=1.14 \Lambda$, orange:

$g=0.7, \sigma=1.04 \Lambda$, in all conditions $r=0.1$. (d) Contribution to $\left(\hat{L}^{0}\right)^{-1}$ from modes off the critical circle, Eq. (6.38).

Their configuration and thus their contribution to the potential is planform dependent. For instance, in the case of the LDP solution the modes come closer to the critical circle, as for the fully symmetric HDP solution. At fifth order there are $n^{3}-2 n^{2}+n$ modes off the critical circle but not all contribute equally. Due to the shape of $\left(\hat{L}^{0}\right)^{-1}$, the closer the modes off the critical circle come to the critical circle, the more they influence the couplings in the amplitude equations. To study the influence of these modes on the coupling function $g_{i j k}$, we mainly concentrate on those modes close to the critical circle which contribute most. We sort the modes according to their distance to the critical circle $\vec{k}_{s}^{(i)}, i=1,2, \ldots$, where the modes $\vec{k}_{s}^{(1)}$ are closest. Note, that the largest contributions to the amplitude equations result not 
(a)

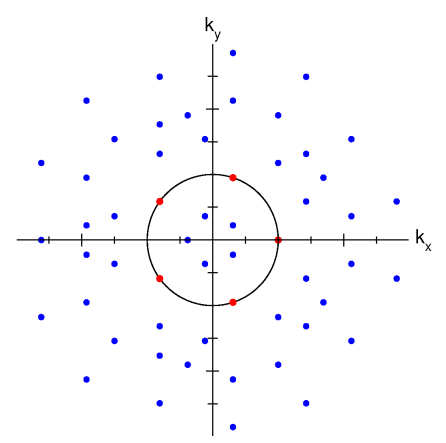

(b)

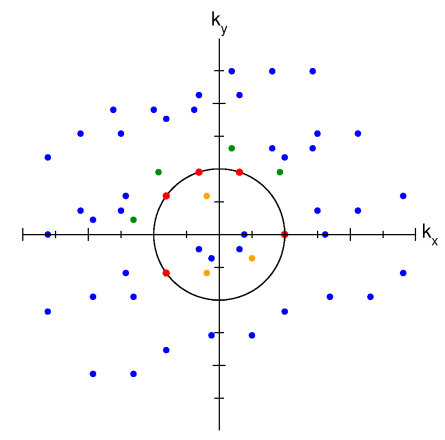

(c)

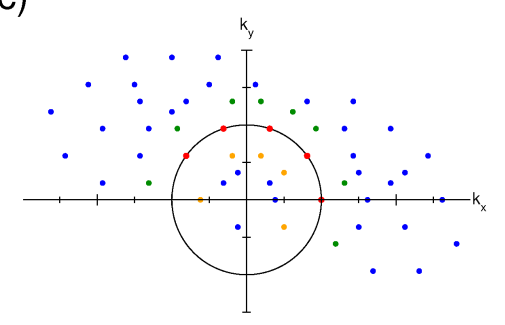

Figure 6.11.: Distribution of modes off the critical circle contributing to the $n=5$ amplitude equations at fifth order, $k_{c}=1$. (a) $l=(1,-1,1,-1,1)$ (b) $l=(1,1,1,-1,1)$ (c) $l=(1,1,1,1,1)$. Active modes are marked in red. Near resonant modes $k_{s}^{(1)}$ and $k_{s}^{(2)}$ are marked in green and orange, respectively.

from the modes closest to the critical circle, but from modes with $\left|k_{s}\right|<k_{c}$. We further define

$$
\begin{aligned}
L_{i} & =\sum_{j} e^{-\imath \vec{k}_{s, j}^{(i)} \vec{x}}\left(\hat{L}^{0}\right)^{-1} e^{\imath \vec{k}_{s, j}^{(i)} \vec{x}}=\sum_{j} \frac{-1}{\left(k_{c}^{2}-\left(\vec{k}_{s, j}^{(i)}\right)^{2}\right)^{2}} \\
& =\sum_{j} \mathcal{L}_{j},
\end{aligned}
$$

where the sum is performed over all modes $k_{s}^{(i)}$ with a fixed distance to the critical circle. We calculate the contribution to the coupling function and thus to the potential that results from these near resonant modes. For all $n=8$ planform solutions we plot $L_{i}$ where we successively included more and more modes off the critical circle, starting with the closest ones. This is shown in Fig. 6.10(d). We observe that the clustering according to $\kappa$ observed in the fifth order potential is already present in the contributions from the near resonant modes.

Modes that have the largest coefficient from $\left(\hat{L}^{0}\right)^{-1}$ are given by $\mathbf{k}_{s}^{(1)}=\mathbf{k}_{i}+\mathbf{k}_{i-2}-\mathbf{k}_{i-1}$ with

$$
\left|\mathbf{k}_{s}^{(1)}\right|=\left|1-2 \cos \left(\frac{\pi}{n}\right)\right|<k_{c}, \quad \mathcal{L}_{1}=\frac{-1}{64} \csc \left(\frac{\pi}{2 n}\right)^{4} \sec \left(\frac{\pi}{n}\right)^{2} .
$$

These modes are marked in orange in Fig. 6.11, $\mathcal{L}_{1}$ grows with $n$ as $n^{4}$ and their number is given by $n$ for a LDP. Therefore $L_{1}$ grows with $n$ as $n^{5}$, see orange line in Fig. 6.12(b). The second largest contribution to $\left(\hat{L}^{0}\right)^{-1}$ results from modes with the minimal distance to the critical circle $\mathbf{k}_{s}^{(2)}=2 \mathbf{k}_{i}-\mathbf{k}_{i-1}$ with

$$
\left|\mathbf{k}_{s}^{(2)}\right|=\sqrt{5-4 \cos \left(\frac{\pi}{n}\right)}>k_{c}, \quad \mathcal{L}_{2}=\frac{-1}{64} \csc \left(\frac{\pi}{2 n}\right)^{4} .
$$

These modes are marked in green in Fig. 6.11. $\mathcal{L}_{2}$ also grows with $n$ as $n^{4}$ and their number is given by $2(n-1)-4 \kappa$. Thus $L_{2}$ grows as $n^{5}$, see green line in Fig. 6.12(b). The length of the modes $k_{s}^{(1)}$ and $k_{s}^{(2)}$ with increasing number of active modes $n$ is shown in Fig. 6.12(a). Their contribution to the amplitude equations in terms of $L_{i}$, see Eq. (6.38), is shown in 

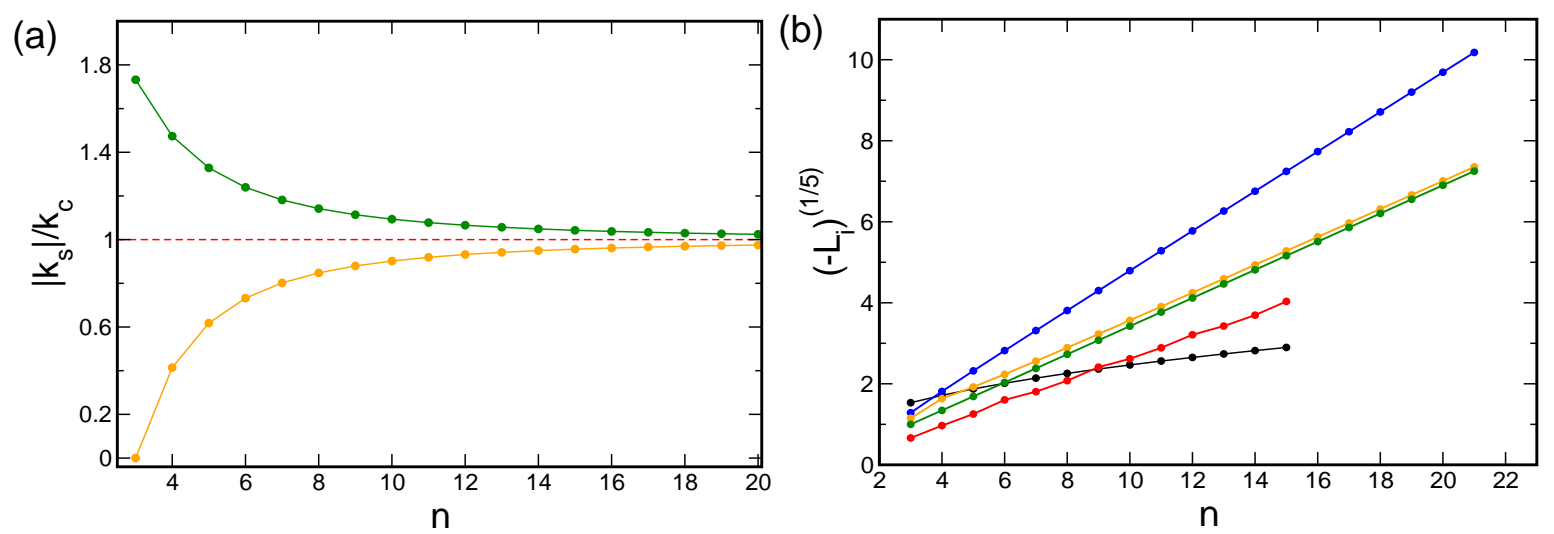

Figure 6.12.: Near resonant modes. (a) Length of modes $\left|\vec{k}_{s}^{(1)}\right|$ (orange) and $\left|\vec{k}_{s}^{(2)}\right|$ (green). (b) $L_{1}$ (orange) and $L_{2}$ (green), see Eq. (6.38). Contribution of all off circle modes $\sum_{i} L_{i}$ (blue). Potential at third $\left(\left(-V_{3}\right)^{(1 / 5)}\right.$, black $)$ and fifth $\left((-V)^{(1 / 5)}\right.$, red) order, parameters: $g=0.9, n=2 \pi \sigma / \Lambda$.

Fig. 6.12(b). Finally, the most distant modes are given by $k_{s}^{(d)}=2 k_{1}-k_{n-1}$ with $\left|k_{s}^{(d)}\right|=3$ and $\mathcal{L}_{d}=-\frac{1}{64}$. We confirmed our analysis by calculating the third (black line) and fifth order (red line) potential with an increasing number of active modes, see Fig. 6.12(b). Indeed the potential at fifth order increases with $n$ as $n^{5}$. Note, when $n$ is a multiple of three triad resonance occur and the potential receives additional contributions. To compensate the fifth order corrections we propose a rescaled model where the bifurcation parameter $r$ has to be model dependent i.e. $r=r(\sigma / \Lambda, g)$. The dependence of the amplitudes $\mathcal{A}$ on the parameter $\sigma$ is negligible if the solution is not close to its stability borders. As $\mathcal{A} \propto \sqrt{r}+r^{3 / 2}$ and the stability borders scale for large $n$ as $n \approx 2 \pi \sigma / \Lambda$, the bifurcation parameter for a given $g$ should scale as $\left(\sqrt{r}+r^{3 / 2}\right)^{6}(\sigma / \Lambda)^{5}=$ const. Furthermore, we revealed the dependence of the potential on the number of flipped mode clusters $\kappa$. Contributions from the inverse linear operator acting on modes off the critical circle are clustered according to $\kappa$, see Fig. 6.10(d). If we take the contribution of the nonlinearity into account, see Fig. 6.10(c), this clustering is preserved. The potential finally is clustered according to $\kappa$ only for $g$ close to 1 . For smaller $g$, modulations in the stationary amplitudes lift the approximate degeneracy in $\kappa$, see Fig. 6.10(a).

\subsection{Stability borders}

In this section we want to answer the question of how much of the multistability of ECP solutions is preserved far from threshold. We show that there are large regions in parameter space in which multistability is preserved even far from threshold. Furthermore, we want to answer the question of how the stability borders of the energetically preferred solutions relate to those which have higher energies. In the following we thus study the stability properties for ECP solutions of the amplitude equations (6.19). To do this we have to consider two types of instabilities namely intrinsic and extrinsic stability. 


\subsubsection{Intrinsic stability}

A planform is intrinsically stable, if it does not become unstable because of the interaction between active modes. Therefore, we check if an $n$ mode planform solution disintegrates into a solution with a lower number of active modes. Let $\mathcal{A}_{i}$ be the stationary solutions. For $A_{i^{-}}=0$ there is a degeneracy in the phases and we can restrict the analysis to amplitude perturbations. For a small perturbation $a_{i}$ around the stationary solution we write

$$
A_{i}=\mathcal{A}_{i}+a_{i}
$$

Inserting this ansatz into the amplitude equations Eq. (6.19) leads to the linear equation

$$
\partial_{t} a_{i}=\sum_{j} M_{i j} a_{j}
$$

with the matrix entries

$$
\begin{aligned}
M_{i j, i \neq j}= & -2 g_{i j} A_{i} A_{j}-2 \sum_{k} g_{i k j} A_{k}^{2} A_{j} A_{i}-2 \sum_{k} g_{i j k} A_{k}^{2} A_{j} A_{i} \\
M_{i i}= & r-2 g_{i i} A_{i}^{2}-\sum_{j} g_{i j} A_{j}^{2} \\
& -2 \sum_{j} g_{i j i} A_{i}^{2} A_{j}^{2}-2 \sum_{j} g_{i i j} A_{i}^{2} A_{j}^{2}-\sum_{j, k} g_{i j k} A_{j}^{2} A_{k}^{2} .
\end{aligned}
$$

Intrinsic stability is given if all eigenvalues of the matrix $M$ are negative. At third order the calculation of eigenvalues simplifies a lot due to permutation symmetry. In this case the stability borders can be derived analytically [50]. At fifth order the stability borders are in general planform dependent. In this case we calculate the eigenvalues of $M$ numerically.

\subsubsection{Extrinsic stability}

Solutions are extrinsically stable if the growth of additional modes is suppressed. To test whether the planform solution could decay into solutions with wavevectors other than the $\vec{k}_{j}$ we introduce a test mode $T$. Near the bifurcation point only test modes on the critical circle have to be considered. For higher order amplitude equations, additional sources for instability can result from test modes off the critical circle which we neglect. We look in the vicinity of a stationary solution $A_{j}$ and assume a small amplitude $T \approx 0$. The third order field is thus composed of the test and active modes

$$
z_{1}=T e^{\imath \vec{k}_{\alpha} \vec{x}}+\sum_{j}^{n} A_{j} e^{\imath \vec{k}_{j} \vec{x}}
$$


with $k_{\alpha}=(\cos \alpha, \sin \alpha) k_{c}$. We insert this ansatz into the third order equation Eq. (6.11). The dynamics of the test mode is then given by

$$
\partial_{t} T=\left(r-\sum_{j}^{n} g\left(\alpha-\alpha_{j}\right)\left|A_{j}\right|^{2}\right) T,
$$

where nonlinear contributions in $T$ are neglected. For the solution $T=0$ to be stable we therefore obtain the condition

$$
\left(r-\sum_{j}^{n} g\left(\alpha-\alpha_{j}\right)\left|A_{j}\right|^{2}\right)<0, \quad \forall \alpha \in[0,2 \pi]
$$

Here we assumed that $\vec{k}_{\alpha} \neq \vec{k}_{j},-\vec{k}_{j}$. In case of the uniform solution $A_{j}=\mathcal{A}$ we get the condition

$$
-g(0) / 2+\sum_{j}^{n} g\left(\alpha_{j}\right)-\sum_{j}^{n} g\left(\alpha-\alpha_{j}\right)<0 .
$$

As shown in [50] for the coupling function Eq. (5.8) the expression Eq. (6.47) takes its maximum value at the intermediate angles $\alpha=\alpha_{i}^{*}=\left|\alpha_{i}-\alpha_{i+1}\right| / 2$. Therefore the extrinsic instability first occurs for modes between two active modes. It is thus sufficient to consider the intrinsic stability of the particular planform with $2 n$ modes. Moreover, at third order all $\alpha_{i}^{*}$ are equivalent. The stability criterion for test modes at $\alpha=\alpha^{*}$ receives corrections at higher orders. To determine extrinsic stability up to fifth order we thus make the following ansatz

$$
\begin{aligned}
& z_{1}=\sum_{j}^{n} A_{j} e^{\imath \vec{k}_{j} \vec{x}}+T e^{\imath \vec{k}_{\alpha} \vec{x}} \\
& z_{3}=\left(\hat{L}^{0}\right)^{-1} N_{3}\left[z_{1}, z_{1}, \bar{z}_{1}\right]+\sum_{j}^{n} B_{j} e^{\imath \vec{k}_{j} \vec{x}}+T^{\prime} e^{\imath \vec{k}_{\alpha} \vec{x}}+T^{\prime \prime} e^{\imath \vec{k}_{\beta} \vec{x}} .
\end{aligned}
$$

In this ansatz we also included a new test mode at a different angle $\beta \neq \alpha$. We insert this ansatz into the equation Eq. (6.15) . The dynamics for the test mode $T^{\prime \prime}$ only reproduces the third order condition Eq. (6.45). The dynamics for $T^{\prime}$ is given by

$$
\begin{aligned}
\partial_{t} T^{\prime}= & r T^{\prime}-\sum_{j}^{n} g\left(\alpha-\alpha_{j}\right)\left|A_{j}\right|^{2} \mathcal{T}^{\prime}-\sum_{j}^{n} g\left(\alpha-\alpha_{j}\right) B_{j} \bar{A}_{j} T \\
& -\sum_{j}^{n} g\left(\alpha-\alpha_{j}\right) A_{j} \bar{B}_{j} T-\sum_{i, j}^{n} g\left(\alpha-\alpha_{i}, \alpha-\alpha_{j}\right)\left|A_{i}\right|^{2}\left|A_{j}\right|^{2} T .
\end{aligned}
$$

We combine equation Eq. (6.49) and Eq. (6.45) by defining the new modes

$$
\widetilde{A}_{j}=\mu A_{j}+\mu^{3} B_{j}, \quad \widetilde{T}=\mu T+\mu^{3} T^{\prime} .
$$



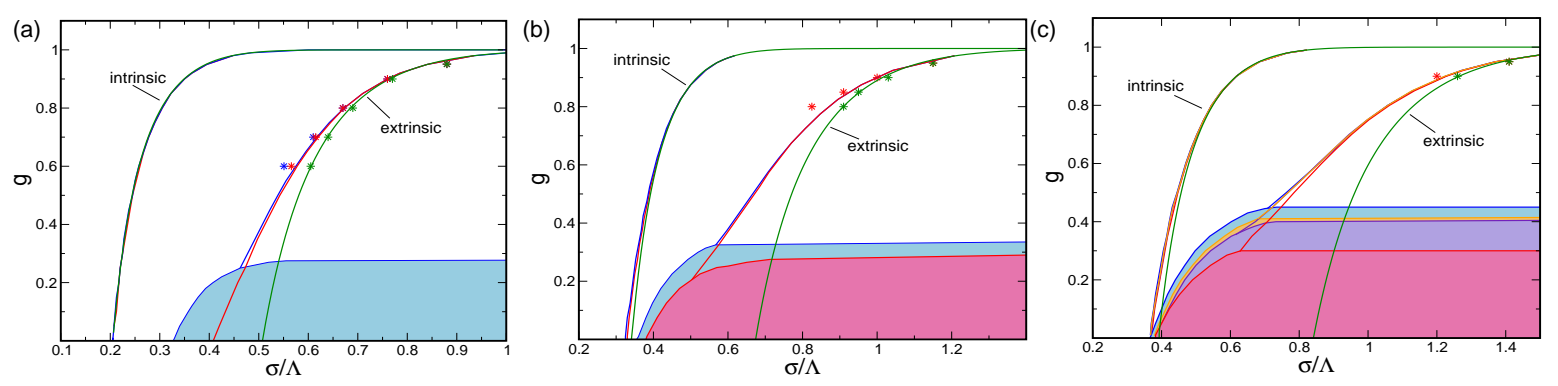

Figure 6.13.: Stability borders at third and fifth order, $r=0.1$ (a) Borders of intrinsic and extrinsic stability for both $n=3$ planforms. Green lines: Third order stability borders, blue lines: $l=(1,1,1)$, red lines: $l=(1-1,1)$. (b) Stability borders for $n=4$ planforms. Blue lines: $l=(1,1,1,1)$, red lines: $l=(1,-1,1,1)$. (c) Stability borders for $n=5$ planforms. Red lines: $l=(1,-1,1,-1,1)$, blue lines: $l=(1,1,1,1,1)$, indigo lines: $l=(1,1,-1,1,1)$ orange lines: $l=(1,-1,1,-1,1)$. Colored regions: amplitude equations of the corresponding planform diverge. Star symbols: Extrinsic stability borders obtained by solving the full field dynamics numerically. Parameters: $T_{f}=5 \cdot 10^{5}, \Gamma=40,256 \times 256$ mesh.

This leads to the stability condition at fifth order

$$
\left(r-\sum_{j}^{n} g\left(\alpha-\alpha_{j}\right)\left|\widetilde{A}_{j}\right|^{2}-\sum_{i, j}^{n} g\left(\alpha-\alpha_{i}, \alpha-\alpha_{j}\right)\left|\widetilde{A}_{i}\right|^{2}\left|\widetilde{A}_{j}\right|^{2}\right)<0 .
$$

As for the leading order it is sufficient to test the intrinsic stability of a planform solution with additional test modes placed at intermediate angles $\alpha^{*}$. However, as the modes at $\alpha^{*}$ and $\alpha^{*}+\pi$ are not equivalent at fifth order we have to consider the system of $3 n$ modes $A_{j}, T_{\alpha^{*}}, T_{\alpha^{*}+\pi}$.

Figure 6.13 shows the intrinsic and extrinsic stability borders of all $n=3, n=4$, and $n=5 \mathrm{ECP}$ solutions. When calculating the fifth order stability borders for the $n=3$ LDP solution, see Fig. 6.13(a), we included the corresponding opposite modes $A_{j^{-}}$, as they are not suppressed at higher order. We observe that the intrinsic stability borders receive only small modifications compared to the third order result. In contrast, near extrinsic stability borders the influence of fifth order corrections is large, and thus the stability borders receive a planform dependent shift towards smaller $\sigma$ values. This shift increases with decreasing $g$ or increasing the number of active modes $n$. In general, planforms which are energetically favored, such as the LDP solutions, receive a larger shift of their extrinsic stability border. We confirmed the shift of the extrinsic stability borders by solving the full field dynamics numerically. As a reference to the third order result we used simulations in which $r=0.01$. The resulting stability borders are marked with star symbols in Fig. 6.13. In line with the results from amplitude equations, the extrinsic stability borders are shifted towards smaller $\sigma$ values. The deviations from the third order results are slightly larger in the full field dynamics than expected from amplitude equations. 


\subsubsection{The question of convergence}

From the stability analysis of the amplitude equations at leading order Eq. (6.13) follows that the model is convergent for all $g>0$ and $\sigma>0$. Fifth order corrections, however, reveal a new type of instability. These corrections enter with a positive sign to the amplitude equations (6.19) due to the shape of the linear operator, see Eq. (6.6). As a result, the corresponding potential can become unbounded and thus the amplitudes diverge. We already observed this type of instability when calculating the phase diagram at fifth order, see Fig. 6.9. When calculating intrinsic and extrinsic stability borders we observe that for small $g$ and for $\sigma$ close to the extrinsic stability borders the amplitude equations diverge. Regions in which the amplitude equations do not converge are colored in Fig. 6.13. The region where the amplitude equations are not convergent is planform dependent. For instance, the $n=3$ LDP solution becomes divergent for small $g$ values whereas the HDP solution, which is energetically not preferred, is stable throughout the whole stability region of this solution. In general the LDP solutions, which receive a larger correction at fifth order, have a larger regime of instability. Remarkably, also for the full field dynamics Eq. (6.1) the convergence is not guaranteed for all parameter values. For $g<1$ the local and non-local parts of the nonlinearity enter with a different sign into the dynamics. With decreasing $g$ the importance of the non-local interaction increases. Thus the field dynamics can diverge for small $g$ and $\sigma$ large. In Fig. 6.13 numerical simulations (star symbols) are thus convergent only if $g$ is not too small. With increasing distance from threshold the region of instability increases. Moreover, as in the case of amplitude equations, the region of instability is planform dependent. HDP patterns as initial conditions are in general more stable than LDP solutions. Although there is a striking similarity between the divergence properties of fifth order amplitude equations and the full field dynamics a detailed comparison is not possible as for large amplitudes higher and higher order corrections to the amplitude equations need to be considered. Attempts to impede this divergence have been done for instance by adding new damping terms such as a non-zero quintic nonlinearity [116].

\subsection{Discussion}

In this chapter we discussed pattern selection by fifth order corrections to the amplitude equations for the uncoupled OP dynamics. Although the leading order amplitude equations give a very good description of the full field dynamics near threshold, higher order corrections have to be considered when studying pattern formation far from threshold. Such corrections are discussed in the literature, see for instance [117, 118, often in the case of a real Swift-Hohenberg equation without long-range interactions where, in contrast to our model, multistability is absent. Numerical simulations indicate that the energetic degeneracy of ECP solutions is lifted far from threshold. Whereas the average pinwheel densities for intermediate times is largely insensitive to an increase in the distance from threshold, pinwheel densities at asymptotic times substantially decrease. In this chapter we could explain these observations by incorporating fifth order corrections to the amplitude equations. To do this we disen- 
tangled the different contributions that can lead to a pinwheel density reduction far from threshold.

First, higher order corrections can change the planform solutions and thus their pinwheel densities for several reasons. Higher order corrections lead to modulations in the stationary amplitudes which are thus in general not uniform anymore. In addition, higher order corrections lead to contributions of modes outside of the critical circle. We showed that both corrections can change the patterns for a given planform solution such that their pinwheel density is slightly reduced. We demonstrated that this effect alone cannot fully explain the reduction in the pinwheel density observed in the full field dynamics. Second, we showed that higher order corrections energetically favor LDP solutions which could additionally reduce the average pinwheel density. However, the energy difference between the LDP and the remaining solutions is rather small. We thus expect additional changes in the energy landscape such that the basin of attraction of LDP solutions is increased.

A feature of the leading order amplitude equations is that all phases of the Fourier modes are left undetermined in the case of ECP solutions. We observe in numerical simulations that for some planform configurations this degeneracy seems to be lifted. Indeed we showed that triad resonances in $n=6,9,12, \ldots$ planforms can lead to additional contributions to the amplitude equations which partially lift the degeneracy of the phases. In addition, we showed that due to these triad resonances opposite modes are no longer suppressed in these planforms. Finally, triad resonances lower the potential of the corresponding ECP solution, which leads to their over-representation in the phase diagram. Higher order corrections moreover change the stability borders of the planform solutions which receive a planform dependent shift. Notably, even for a substantial distance from threshold there are large regions in the phase diagram in which multistability is preserved.

In addition, we identified a secondary instability. From the stability analysis of the amplitude equations at leading order Eq. (6.13) follows that the model is in general convergent. However, the stability of the system with higher order terms Eq. (6.19) is not guaranteed. As it turns out, for finite $r$, there is a large region in the $(\sigma / \Lambda, g)$-plane where the system is not stable. Indeed also the full field dynamics is not convergent for large $\sigma / \Lambda$ values and shows the same planform dependence of this instability [116].

What does this chapter tells us about the practicability of the OP model used? Important theoretical conclusions of the model are drawn in the large $n$ (i.e. large $\sigma$ ) limit, for instance that the average pinwheel density approaches $\rho=\pi$. By inspection of the distribution of near resonant terms we showed that there is a strong $\sigma$ dependence on the strength of higher order corrections and thus on the instability of the dynamics. We thus propose a rescaled model where the bifurcation parameter is itself model parameter dependent. Such a model is assumed to have a uniform stability range and a well defined large $\sigma$ limit.

It is hard to estimate the value of the bifurcation parameter in physiological maps. The power spectrum of such maps is not composed of distinct Fourier modes but is rather smeared out around the critical circle [55, 75, 79]. However, there are indirect estimations. If the formation of cortical maps takes place close to threshold, the predicted multistability should be 
preserved to a large extent. Indeed experimental findings suggest the existence of multistability. Perturbing the system by intracortical microstimulation leads to a substantial and long-lasting reorganization of the OP map [88. These experiments can be interpreted as a switching between several different equilibrium states [16]. 


\section{Pattern Selection with Broken Permutation Symmetry}

In this chapter we investigate a second mechanism for pattern selection. The proposed permutation symmetry Eq. (5.5) of the OP dynamics guarantees the instability of unrealistic 'orientation scotoma' solutions and leads to the massive multistability of ECP solutions. However, the biological basis of this symmetry is not clear. To provide a general theory of OP map development with translation, rotation, and orientation-shift symmetry we break permutation symmetry. We show that the way we break this symmetry still guarantees the instability of orientation scotoma solutions. We further demonstrate that breaking permutation symmetry can lead to realistic pinwheel densities even when considering pattern formation far from threshold. Breaking permutation symmetry leads to modulations in the stationary amplitudes already at leading order. We show that these modulations in general can increase the pinwheel density of the corresponding planform. Moreover, breaking permutation symmetry partially lifts the multistability of ECP solutions and energetically selects certain solutions. Because permutation symmetry leads to a strong mode-antimode competition, the ECP solutions form the dominant class of solutions in this case. Other solutions, however, can exist that may supersede the ECP solutions in case of broken permutation symmetry. In addition to ECP solutions we identify and characterize two additional classes of stationary solutions. One of these classes actually becomes relevant in case of broken permutation symmetry. In particular, we demonstrate that solutions of this class can even become the energetic ground state.

We start this chapter with a derivation of the permutation symmetric model Eq. (3.4) from a coupled model for the OP map and a field describing the cortical connectivity. Next, we illustrate the different ways to break the permutation symmetry.

\subsection{Breaking the permutation symmetry}

In Chapter 5 we introduced non-local interaction terms for the dynamics of the OP map. In this section we show that these non-local terms are obtained by a coupled model for the development of the OP map and long-range horizontal connections. This derivation follows [119]. Next, we show how to break the permutation symmetry by introducing a disbalance between the two non-local interaction terms. Consider the coupled dynamics of the OP map and a field $W(\mathbf{x}, \mathbf{y})$ which describes the strength of long-range horizontal 
(a)

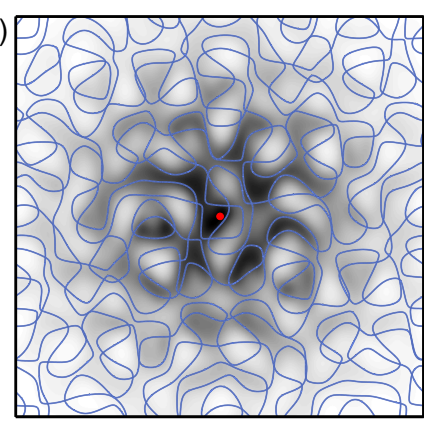

(b)

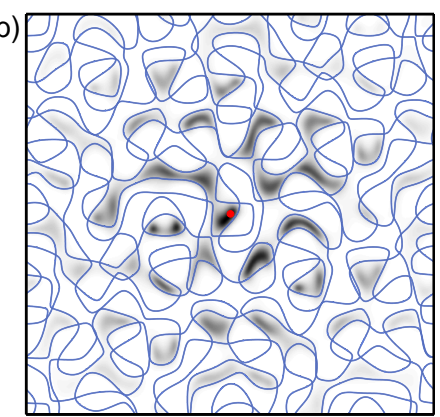

Figure 7.1: Long-range

connections. Blue lines: zero contours of $\operatorname{Re}(z)$ and $\operatorname{Im}(z)$, darker regions correspond to stronger long-range connectivity, i.e. larger $W(\mathbf{x}, \mathbf{y})$. Red dot: position $y$. (a) $\sigma_{x}=2 \Lambda, \sigma_{z}=1.5$ (b) $\sigma_{x}=2 \Lambda, \sigma_{z}=0.5$.

connections. The dynamics is assumed to be of the form

$$
\begin{aligned}
\partial_{t} z(\mathbf{x}) & =F_{z}[z]+F_{z W}[z, W] \\
\tau \partial_{t} W(\mathbf{x}, \mathbf{y}) & =F_{W z}[z, W]
\end{aligned}
$$

where $\mathbf{x}$ and $\mathbf{y}$ correspond to the position of two neurons in V1 and $\tau$ is the time scale on which the connections develop. We assume that long-range connections have a typical width $\sigma_{x}$. The simplest uncoupled dynamics for $W$ is then given by

$$
\tau \partial_{t} W(\mathbf{x}, \mathbf{y})=-W(\mathbf{x}, \mathbf{y})+\frac{1}{2 \pi \sigma_{x}^{2}} e^{-|\mathbf{x}-\mathbf{y}|^{2} /\left(2 \sigma_{x}^{2}\right)} e^{-|z(x)-z(y)|^{2} /\left(2 \sigma_{z}^{2}\right)},
$$

with the stationary state

$$
W(\mathbf{x}, \mathbf{y})=\frac{1}{2 \pi \sigma_{x}^{2}} e^{-|\mathbf{x}-\mathbf{y}|^{2} /\left(2 \sigma_{x}^{2}\right)} e^{-|z(x)-z(y)|^{2} /\left(2 \sigma_{z}^{2}\right)} .
$$

With decreasing $\sigma_{z}$ the long-range connections become more and more selective to the preferred orientation, see Fig. 7.1. The uncoupled dynamics of the OP map is given by

$$
F_{z}[z]=\hat{L} z-g_{z z}|z(\mathbf{x})|^{2} z(\mathbf{x})
$$

As we have seen in the previous chapters the stationary patterns of the uncoupled dynamics are pinwheel free stripes. The coupling term is assumed to be of the form

$$
F_{z W}[z, W]=g_{z W} \int d^{2} y(z(\mathbf{x})+\beta z(\mathbf{y})) W(\mathbf{x}, \mathbf{y}) .
$$

Eq. (7.5) is a general linear coupling term between neurons at cortical position $\mathbf{x}$ and $\mathbf{y}$. The parameter $\beta$ thus weights changes at position $\mathbf{x}$ according to the OP at position $\mathbf{y}$. We will see in the following that the special choice of $\beta=-1$ corresponds to the permutation symmetric dynamics presented in Chapter 5. Experiments show that during the evolution of long-range connections changes in the OP map are small. Therefore we can assume that the dynamics of $W(\mathbf{x}, \mathbf{y})$ evolves for a static field $z$. We therefore can adiabatically eliminate $W(\mathbf{x}, \mathbf{y})$, leading to an effective dynamics for the OP map. Near threshold $z \approx 0$ and we can perform a series 
expansion of Eq. (7.5). The resulting effective linear part is given by

$$
\hat{L}_{\mathrm{eff}}=\hat{L} z(\mathbf{x})+g_{z W} z(\mathbf{x})-\frac{\beta g_{z W}}{2 \pi \sigma_{x}^{2}} \int d^{2} y e^{-|\mathbf{x}-\mathbf{y}|^{2} /\left(2 \sigma_{x}^{2}\right)} z(\mathbf{y}) .
$$

The second term in $\hat{L}_{\text {eff }}$ just rescales the bifurcation parameter $r$. The third term leads to a modulation of the spectrum. This spectrum still has a maximum at a (shifted) critical wavelength. For large $\sigma_{x}$ this modulation is small and we can use this linear operator in the same way we use the Swift-Hohenberg operator. The effective cubic part is given by

$$
\begin{aligned}
N_{\mathrm{eff}}[z, z, \bar{z}]= & -\left(\frac{g_{z W}}{2 \sigma_{z}^{2}}+g_{z z}\right)|z(\mathbf{x})|^{2} z(\mathbf{x}) \\
& \frac{g_{z W}}{4 \pi \sigma_{x}^{2} \sigma_{z}^{2}} \int d^{2} y e^{-|\mathbf{x}-\mathbf{y}|^{2} /\left(2 \sigma_{x}^{2}\right)}\left((\beta-1) z(\mathbf{x})|z(\mathbf{y})|^{2}+\beta \bar{z}(\mathbf{x}) z(\mathbf{y})^{2}\right. \\
& \left.(1-\beta)|z(\mathbf{x})|^{2} z(\mathbf{y})-\beta|z(\mathbf{y})|^{2} z(\mathbf{y})+z(\mathbf{x})^{2} \bar{z}(\mathbf{y})\right) .
\end{aligned}
$$

The coupling function resulting from this nonlinearity is given by

$$
\begin{aligned}
g(\alpha)= & 2 g_{z z}+\frac{3 g_{z W}}{2 \sigma_{z}^{2}}-\frac{\beta g_{z W}}{2 \sigma_{z}^{2}}-\frac{2(1-\beta) g_{z W}}{\sigma_{z}^{2}} e^{-(1 / 2) \sigma_{x}^{2} k_{c}^{2}} \\
& +\frac{g_{z W}}{2 \sigma_{z}^{2}}\left((1-\beta) e^{-\sigma_{x}^{2} k_{c}^{2}(1-\cos \alpha)}-2 \beta e^{-\sigma_{x}^{2} k_{c}^{2}(1+\cos \alpha)}\right) .
\end{aligned}
$$

Thus only two of the five non-local terms lead to an angle dependent coupling function. In case of $\beta=-1$ and $\sigma_{x}$ large we obtain the permutation symmetric model introduced in Chapter 5. For $\beta \neq-1$ permutation symmetry is broken and $g(\alpha) \neq g(\alpha+\pi)$. We adapt this model by introducing a disbalance $\epsilon$ between the two non-local interaction terms. We further introduce different widths for the two non-local terms $\sigma_{1}$ and $\sigma_{2}$. The cubic nonlinearity we consider in the following therefore reads

$$
\begin{aligned}
N_{3}[z, z, \bar{z}]= & (g-1)|z(x)|^{2} z(x)+ \\
& \frac{2-g}{2 \pi \sigma_{1}^{2}} \int d^{2} y e^{-|x-y|^{2} / 2 \sigma_{1}^{2}} z(x)|z(y)|^{2}+ \\
& \frac{\epsilon}{2} \frac{2-g}{2 \pi \sigma_{2}^{2}} \int d^{2} y e^{-|x-y|^{2} / 2 \sigma_{2}^{2}} \bar{z}(x) z(y)^{2} .
\end{aligned}
$$



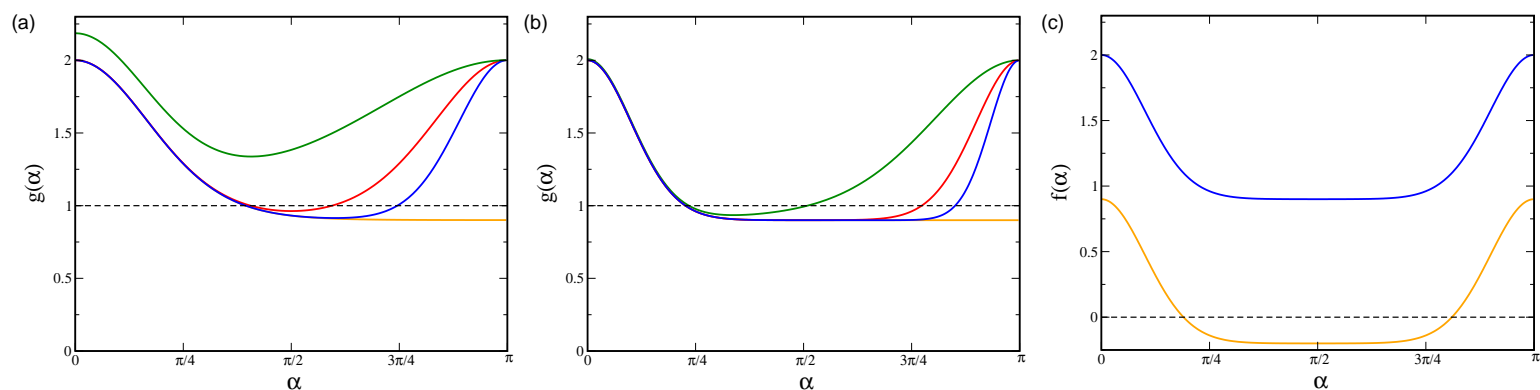

Figure 7.2.: Coupling functions, Eq. (7.11) obtained from the nonlinearity Eq. (7.9)). (a,b) $g(\alpha)$, red: $\pi$-periodic coupling function for $\sigma_{1}=\sigma_{2}, \epsilon=1$, green: $\sigma_{2}=0.5 \sigma_{1}, \epsilon=1$, blue: $\sigma_{2}=1.5 \sigma_{1}, \epsilon=1$, orange: $\epsilon=0$. (a) $g=0.9, \sigma_{1}=0.3 \Lambda$, (b) $g=0.9, \sigma_{1}=0.5 \Lambda$. (c) $f(\alpha)$, blue: $\epsilon=1, \sigma_{1}=0.5 \Lambda$, orange: $\epsilon=0, g=0.9, \sigma_{1}=\sigma_{2}=0.5 \Lambda$.

For $\sigma_{1} \neq \sigma_{2}$ or $\epsilon \neq 1$ permutation symmetry is broken. The corresponding coupling coefficients are given by

$$
\begin{aligned}
g_{i j} & =g+(2-g)\left(e^{-\frac{1}{2} \sigma_{1}^{2}\left(\vec{k}_{i}-\vec{k}_{j}\right)^{2}}+\epsilon e^{-\frac{1}{2} \sigma_{2}^{2}\left(\vec{k}_{i}+\vec{k}_{j}\right)^{2}}\right) \\
g_{i i} & =1+\frac{\epsilon}{2}(2-g) e^{-2 \sigma_{2}^{2}} \\
g_{i j^{-}} & =g+(2-g)\left(e^{-\frac{1}{2} \sigma_{1}^{2}\left(\vec{k}_{i}+\vec{k}_{j}\right)^{2}}+\epsilon e^{-\frac{1}{2} \sigma_{2}^{2}\left(\vec{k}_{i}-\vec{k}_{j}\right)^{2}}\right) \\
g_{i i^{-}} & =2+(2-g) e^{-2 \sigma_{1}^{2}} \\
f_{i j} & =g(2-\epsilon)+2(\epsilon-1)+(2-g)\left(e^{-\frac{1}{2} \sigma_{1}^{2}\left(\vec{k}_{i}-\vec{k}_{j}\right)^{2}}+e^{-\frac{1}{2} \sigma_{1}^{2}\left(\vec{k}_{i}+\vec{k}_{j}\right)^{2}}\right) .
\end{aligned}
$$

Note, that in the permutation symmetric case $g_{i j^{-}}=g_{i j}$ and $2 g_{i i}=g_{i i^{-}}$.

As in the permutation symmetric case we define coupling functions which are given by

$$
\begin{aligned}
& g(\alpha)=g+(2-g)\left(e^{-\sigma_{1}^{2}(1-1 \cos \alpha)}+\epsilon e^{-\sigma_{2}^{2}(1+\cos \alpha)}\right) \\
& f(\alpha)=g(2-\epsilon)+2(\epsilon-1)+(2-g)\left(e^{-\sigma_{1}^{2}(1-1 \cos \alpha)}+e^{-\sigma_{1}^{2}(1+\cos \alpha)}\right) .
\end{aligned}
$$

The coupling function $f(\alpha)$ is in general $\pi$-periodic. In case of $\epsilon=1$ it is further independent of the permutation symmetry breaking. The coupling functions in case of $\epsilon=0$ are shown in Fig. 7.2 (orange lines). The typical shape of $g(\alpha)$ and $f(\alpha)$ in the symmetric and symmetry broken case is shown in Fig. 7.2. We first consider the extreme case of permutation symmetry breaking which is given for $\epsilon=0$. Compared to the case $\epsilon=1$ there is only a single peak of $g(\alpha)$ at $\alpha=0$ while near $\alpha=\pi$ the function is flat. Moreover, the function $f(\alpha)$ can become negative for $\epsilon=0$, see Fig. [7.2(c). The loss of the second peak at $\alpha=\pi$ and a negative $f(\alpha)$ have dramatic consequences for the stability of ECP solutions. We will show in Section 7.2 that this leads to stable orientation scotoma solutions. To avoid such unrealistic solutions we therefore set $\epsilon=1$ in the following. For $\epsilon=1$ the coupling function $g(\alpha)$ has two peaks, at $\alpha=0$ and $\alpha=\pi$ where $f(\alpha)>0$ for $g>0$. 


\subsection{The extended solution set}

In the permutation symmetric case the ECP solution family is the only relevant class of solutions as a strong mode-antimode competition leads to a strong energetic preference for ECP solutions, see Fig. 5.1] If this mode-antimode competition is relaxed by breaking permutation symmetry, other classes of solutions can become relevant. In this section we study two additional solution classes and show that one class indeed can become relevant in case of broken permutation symmetry. Some of its solutions can even become the energetic ground state. In the following we consider the leading order amplitude equations

$$
\partial_{t} A_{i}=r A_{i}-\sum_{j} g_{i j}\left|A_{j}\right|^{2} A_{i}-\sum_{j} f_{i j} A_{j} A_{j^{-}} \bar{A}_{i^{-}}
$$

We split the amplitudes into their absolute values and their phases $A_{i}=\mathcal{A}_{i} e^{\imath \phi_{i}}$ which leads to

$$
\begin{aligned}
\partial_{t} \mathcal{A}_{i} & =r \mathcal{A}_{i}-\sum_{j} g_{i j} \mathcal{A}_{j}^{2} \mathcal{A}_{i}-\sum_{j} f_{i j} \mathcal{A}_{j} \mathcal{A}_{j^{-}} \mathcal{A}_{i^{-}} \cos \left(\Phi_{j}-\Phi_{i}\right) \\
\partial_{t} \phi_{i} & =-\sum_{j} f_{i j} \frac{\mathcal{A}_{j} \mathcal{A}_{j^{-}} \mathcal{A}_{i^{-}}}{\mathcal{A}_{i}} \sin \left(\Phi_{j}-\Phi_{i}\right)
\end{aligned}
$$

with $\Phi_{j}=\phi_{j}+\phi_{j^{-}}$. In the following we discuss three classes of stationary solutions to Eq. (7.12). First, for the ECP solutions we study the impact of permutation symmetry breaking on these solutions. Next, we consider two solution classes in which the opposite modes are non-zero.

\subsubsection{Essentially complex planforms}

In case of ECP solutions opposite modes vanish, $A_{j^{-}}=0$, and stationary solutions to the amplitude equations Eq. (17.12) are given by

$$
\mathcal{A}_{i}=\sqrt{r \sum_{j}\left(g^{-1}\right)_{i j}},
$$

where $g^{-1}$ is the inverse of the coupling matrix $g_{i j}$. In the permutation symmetric case the matrix $g$ is a circulant matrix, the stationary amplitudes are uniform, and Eq. (7.14) simplifies to $\mathcal{A}=\sqrt{r / \sum_{j} g_{i j}}$. In the permutation symmetry broken case the matrix $g_{i j}$ is planform dependent, as $g_{i j^{-}} \neq g_{i j}$. Moreover, $g$ is in general no longer a circulant matrix. Thus except for symmetric planform configurations the stationary amplitudes are no longer uniform when permutation symmetry is broken. The stationary amplitudes for certain ECP solutions are displayed in Fig. 7.3, where we use the natural logarithm to describe the degree of permutation symmetry breaking. Permutation symmetry is broken in both directions i.e. $\sigma_{2}<\sigma_{1}$ and $\sigma_{2}>\sigma_{1}$. For symmetric planform configurations such as the $n=3$ and $n=5 \mathrm{HDP}$ solutions the stationary solutions stay uniform (black dashed lines in Fig. $7.3(\mathrm{a}, \mathrm{c})$ ). For $n=8$ there is 
no such symmetric planform configuration and thus no solution with uniform amplitudes. In general, amplitude modulations are larger for LDP than for HDP solutions. With increasing ratio $\sigma_{2} / \sigma_{1}$ the amplitudes become more and more modulated until a saturation occurs. The shape of the amplitude modulations is shown in Fig. 7.3 (b,d,f,h). When we compare these modulations to those obtained from permutation symmetric amplitude equations at fifth order, see Fig. 6.4, we observe that the shape of the modulations is reversed. 

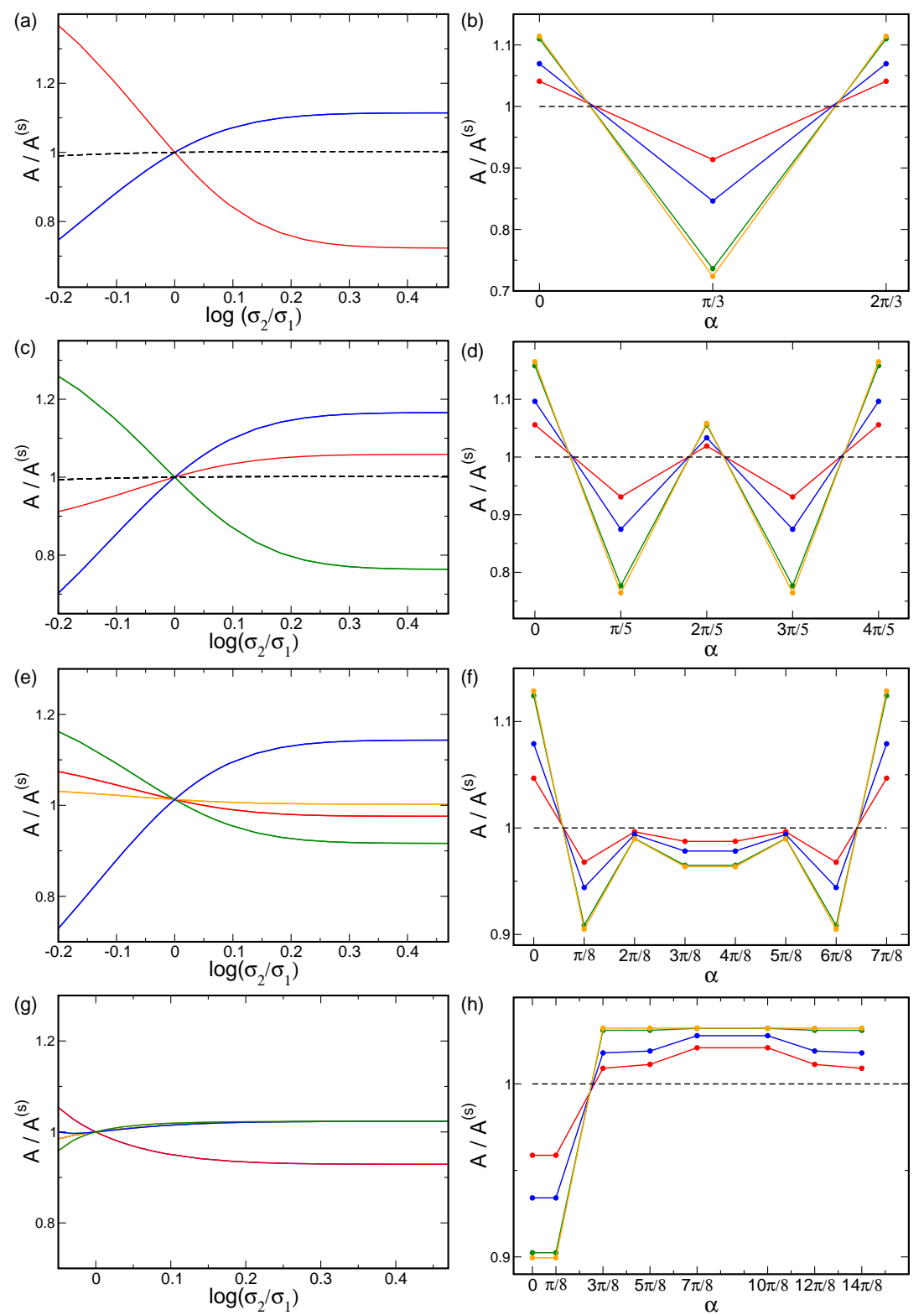

Figure 7.3.: Stationary amplitudes. $A$ amplitudes in the symmetry broken case Eq. (7.14), $A^{(s)}$ amplitudes in the symmetric case. (a,b) $n=3, \sigma_{1}=0.5 \Lambda$, colored lines: LDP, black dashed lines: HDP, $(\mathbf{c}, \mathbf{d}) n=5, \sigma_{1}=0.8 \Lambda$, colored lines: LDP, black dashed lines: $\operatorname{HDP},(\mathbf{e}, \mathbf{f}) n=8, \sigma_{1}=1.3 \Lambda$, LDP $(\mathbf{g}, \mathbf{h}) n=8, \sigma_{1}=1.3 \Lambda$, HDP. Right panel: Modulation of amplitudes as a function of the angle $\alpha$ on the critical circle, $\sigma_{2}=1.05 \sigma_{1}$ (red), $\sigma_{2}=1.1 \sigma_{1}$ (blue), $\sigma_{2}=1.3 \sigma_{1}$ (green), $\sigma_{2}=1.5 \sigma_{1}$ (orange). In all conditions $g=0.98$. 


\subsubsection{Circular phase progression solutions}

Next, we consider uniform solutions with $\mathcal{A}_{j}=\mathcal{A}_{j^{-}}=\mathcal{A}$. We will see in the following that some uniform solutions can become stable in the permutation symmetric as well as in the broken case. Moreover, in the symmetry broken case, one of these solutions can even become the energetic ground state. In case of uniform amplitudes we can combine the equations for the phases $\phi_{j}$ and $\phi_{j^{-}}$in Eq. (7.13) and get

$$
\partial_{t} \Phi_{i}=-2 \mathcal{A}^{2} \sum_{j} f_{i j} \sin \left(\Phi_{j}-\Phi_{i}\right)
$$

One set of stationary solutions to Eq. (7.15) is obtained by

$$
\Phi_{j}^{(\Delta)}=\frac{2 \pi j}{n} \Delta+2 \phi_{0}
$$

with $\Delta$ an integer and $\phi_{0}$ an arbitrary phase. Due to the constant increment in the phase we refer to these solutions as Circular Phase Progression (CPP) solutions. CPP states can be considered as a generalization of the rhombic and hexagonal pinwheel crystals which we encountered in Chapter 4 . Their stationary amplitudes are given by

$$
\mathcal{A}^{(\Delta)}=\sqrt{\frac{r}{\sum_{j}\left(g_{i j}+f_{i j} \cos \left(\Phi_{i}-\Phi_{j}\right)\right)}} .
$$

Figure 7.4 illustrates the different CPP solutions. Note, as in the case of uniform solutions discussed in Chapter 5 , the solutions $\Phi^{(\Delta)}$ and $\Phi^{(n-\Delta)}$ are equivalent in the sense that they have identical stationary amplitudes $\mathcal{A}^{(\Delta)}=\mathcal{A}^{(n-\Delta)}$ and stability properties. Identical pinwheel densities are obtained when averaging the remaining free phases. The special case of $\Delta=0$ corresponds to orientation scotoma solutions, as their OP map is selective only for the two orthogonal orientations $\vartheta=\phi_{0}$ and $\vartheta=\phi_{0}+\pi / 2$. These solutions are higher mode generalizations of the orientation scotoma stripe solution discussed in Chapter 4. In particular, for $\phi_{0}=0$ the solution is real. For $\Delta>0$ all solutions are selective to all preferred orientations. Their stability properties are discussed in Section 7.5. For $n=1,2,3$ the OP map is spatially periodic whereas for $n>3$ the patterns become spatially irregular. All $\Delta>0$ solutions are highly isotropic.

If the CPP solution class should be relevant in models of visual cortical development the stability of the $\Delta=0$ solution should be excluded. To check this we linearize Eq. (7.15) around $\Phi_{j}=2 \phi_{0}+\varphi_{j}$. This leads to

$$
\begin{aligned}
\partial_{t} \varphi_{i} & =-2 \mathcal{A}^{2} \sum_{j} f_{i j}\left(\varphi_{j}-\varphi_{i}\right) \\
& =2 \mathcal{A}^{2} \sum_{j} M_{i j} \varphi_{j}
\end{aligned}
$$


(a)
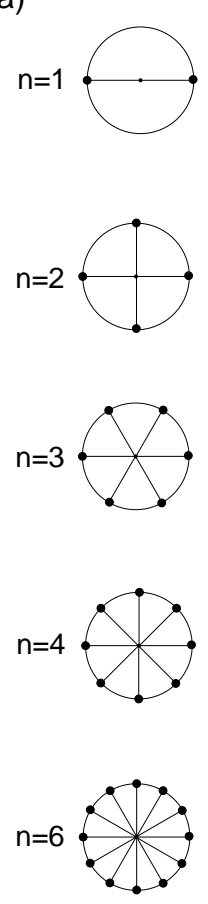

$\Delta=0$
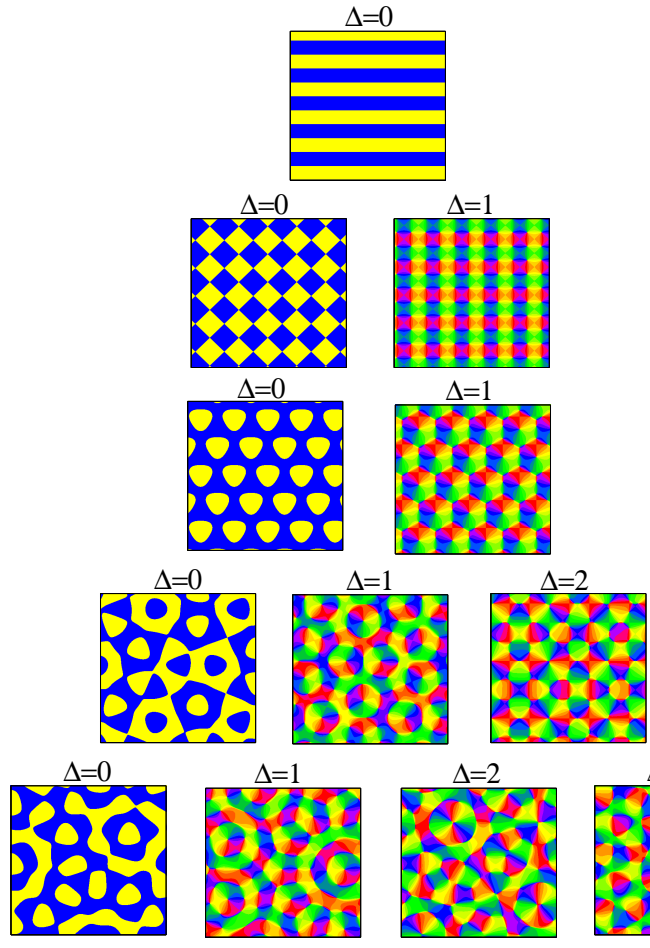

(b)

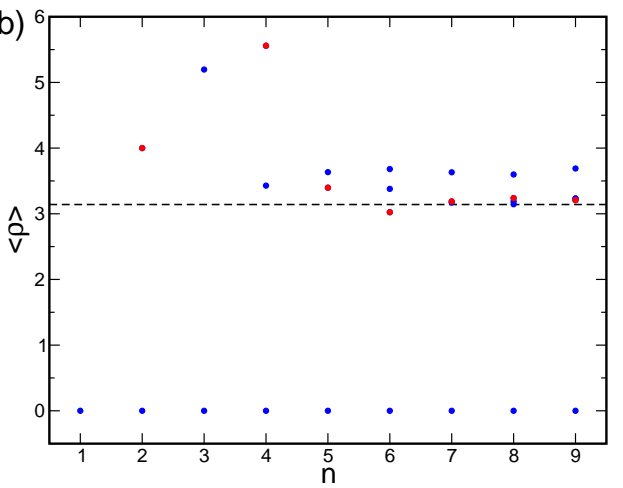

(c)

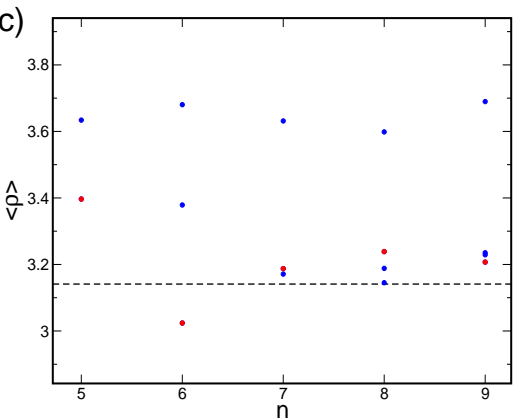

Figure 7.4.: CPP solutions. (a) All CPP solutions, Eq. (7.16), for a given $n$. The diagrams to the left of each pattern display the position of the wavevectors of active modes on the critical circle. $(\mathbf{b}, \mathbf{c})$ Average pinwheel densities for all CPP solutions with a given $n$. Solutions that can become stable in the model Eq. (7.9) are marked in red.

with $M_{i j}=-f_{i j}+\delta_{i j} \sum_{k} f_{i k}$. The matrix $f_{i j}$ is a circulant matrix, independent of the existence of permutation symmetry. The eigenvalues are thus given by

$$
\begin{aligned}
\lambda_{l} & =\sum_{j} M_{0 j} \cos (2 \pi j l / n) \\
& =\sum_{j} f_{0 j}-\sum_{j} \cos (2 \pi j l / n) f_{0 j} .
\end{aligned}
$$

One eigenvalue is zero $\left(\lambda_{0}=0\right)$. If $f_{0 j}>0, \forall j$ the remaining eigenvalues are positive and thus the $\Delta=0$ solution unstable. However, if some entries of $f_{0 j}$ become negative this solution can become stable. Another criterion for the stability of the orientation scotoma solutions is obtained from the dynamics of the amplitudes which in case of Eq. (7.16) is given by

$$
\partial_{t} \mathcal{A}_{i}=r \mathcal{A}_{i}-\sum_{j} g_{i j} \mathcal{A}_{j}^{2} \mathcal{A}_{i}-\sum_{j} f_{i j} \mathcal{A}_{j} \mathcal{A}_{j^{-}} \mathcal{A}_{i^{-}}
$$


We study amplitude perturbations $\mathcal{A}_{i}=\mathcal{A}+a_{i}$ with $\mathcal{A}^{2}=r / \sum_{j}\left(g_{i j}+f_{i j}\right)$. Their linearized dynamics is given by

$$
\begin{aligned}
\partial_{t} a_{i} & =r a_{i}-\mathcal{A}^{2} \sum_{j} g_{i j}\left(2 a_{j}+a_{i}\right)-\mathcal{A}^{2} \sum_{j} f_{i j}\left(a_{j}+a_{j^{-}}+a_{i^{-}}\right) \\
& =-2 \mathcal{A}^{2} \sum_{j} g_{i j} a_{j}-\mathcal{A}^{2} \sum_{j} f_{i j}\left(a_{j}+a_{j^{-}}+a_{i^{-}}\right)+\mathcal{A}^{2} \sum_{j} f_{i j} a_{i} \\
& =-2 \mathcal{A}^{2} \sum_{j} g_{i j} a_{j}-\mathcal{A}^{2} \sum_{j}\left(f_{i j}+f_{i j^{-}}\right) a_{j}-\mathcal{A}^{2}\left(a_{i^{-}}-a_{i}\right) \sum_{j} f_{i j} \\
& =-\mathcal{A}^{2} \sum_{j} M_{i j} a_{j}
\end{aligned}
$$

with the stability matrix $M_{i j}=2 g_{i j}+f_{i j}+f_{i j^{-}}+\left(\delta_{i j^{-}}-\delta_{i j}\right) \sum_{k} f_{i k}$. The stability matrix is a circulant matrix. The solutions are unstable if there is an index $j$ with $M_{i j}>M_{i i}$. This can be shown with the use of the quadratic form $Q=\sum_{i, j} a_{i} M_{i j} a_{j}$. If all eigenvalues of the symmetric matrix $M$ are positive, then $Q>0$ for arbitrary $a_{i}$. However, if $M_{i j}>M_{i i}$ for one pair $(i, j)$ of modes, then choosing $a_{i}=1, a_{j}=-1$, and $a_{k}=0$ for $k \neq i, j$ leads to $Q=M_{i i}-M_{i j}<0$. Thus if $M_{i j}>M_{i i}$ the $\Delta=0$ solution cannot be stable. In particular, for the mode configuration $\left(i, i^{-}\right)$this leads to

$$
\begin{aligned}
M_{i i^{-}} & >M_{i i} \\
g_{i i^{-}}+\sum_{k} f_{i k} & >g_{i i} .
\end{aligned}
$$

The solution is thus already unstable if $g_{i i-}>g_{i i}$, i.e. $g(\pi)>g(0) / 2$. In case of permutation symmetry this condition is always fulfilled, as in this case $g(\alpha)=g(\alpha+\pi)$. If we break permutation symmetry the $\Delta=0$ solutions in general can become stable. A simple model showing this behavior is given by the extreme case of permutation symmetry breaking i.e. where the second non-local interaction term in Eq. (7.9) is set to zero, $\epsilon=0$. In contrast, if $\epsilon=1$ the criterion $g_{i i-}>g_{i i}$ is fulfilled even if $\sigma_{1} \neq \sigma_{2}$ and thus the $\Delta=0$ solutions are in general unstable.

\subsubsection{Binary phase planforms}

Another set of solution families to Eq. (7.13) is obtained by requiring $\sin \left(\Phi_{i}-\Phi_{j}\right)=0, \forall(i, j)$. These stationary solutions thus have

$$
\Phi_{j}^{*}=2 \phi_{0}+\frac{1}{2}\left(l_{j}+1\right) \pi
$$

with $l_{j}= \pm 1$. So there are $2^{n}$ of these solutions. However, many of them can be transferred into each other by rotations or reflections as it is the case for ECP solutions. We refer to these solutions as the Binary Phase Planforms BPP. This family of solutions contains some of the uniform solutions discussed before. For instance $l_{j}=(-1,-1,-1, \ldots,-1)$ and $l_{j}=$ $(1,-1,1,-1,1,-1, \ldots)$ are uniform solutions that are identical to the $\Delta=0$ and $\Delta=n / 2 \mathrm{CPP}$ 
solutions. For the remaining solutions the stationary amplitudes are not uniform. However, due to symmetry, the amplitude equations for $A_{j}$ and $A_{j}$ - are identical. The BPP solutions are model dependent in the presence and absence of permutation symmetry and thus the pinwheel density depends on the model parameters $g, \sigma_{1}, \sigma_{2}$. We solve Eq. (7.12) numerically. The resulting stationary amplitudes and corresponding OP maps for BPP solutions are displayed in Fig. 7.5. Stationary amplitudes are normalized by the amplitude of the $\Delta=0$ uniform solution $A_{\Delta=0}$. To illustrate amplitude modulations consider the phase configuration $l_{i}=$ $-1, i \neq u, i=1, \ldots, n$ and $l_{u}=1$ which is shown in blue in Fig. 7.5. The corresponding amplitude equations read

$$
\begin{aligned}
\partial_{t} \mathcal{A}_{i} & =r \mathcal{A}_{i}-\sum_{j} g_{i j} \mathcal{A}_{j}^{2} \mathcal{A}_{i}-\sum_{j \neq u} f_{i j} \mathcal{A}_{j} \mathcal{A}_{j^{-}} \mathcal{A}_{i^{-}}+f_{i u} \mathcal{A}_{u} \mathcal{A}_{u^{-}} \mathcal{A}_{i^{-}} \\
\partial_{t} \mathcal{A}_{u} & =r \mathcal{A}_{u}-\sum_{j} g_{u j} \mathcal{A}_{j}^{2} \mathcal{A}_{u}+\sum_{j \neq u} f_{u j} \mathcal{A}_{j} \mathcal{A}_{j^{-}} \mathcal{A}_{i^{-}}
\end{aligned}
$$

From these equations it becomes clear that the stationary amplitude $\mathcal{A}_{u}$ is substantially larger than the remaining amplitudes $\mathcal{A}_{j}$ if $f_{i j}>0, \forall i, j$. This leads to a more stripe-like pattern with a relatively low pinwheel density. The other BPP solutions, however, become more and more isotropic the more phase relations with $l_{j}=1$ are included. 

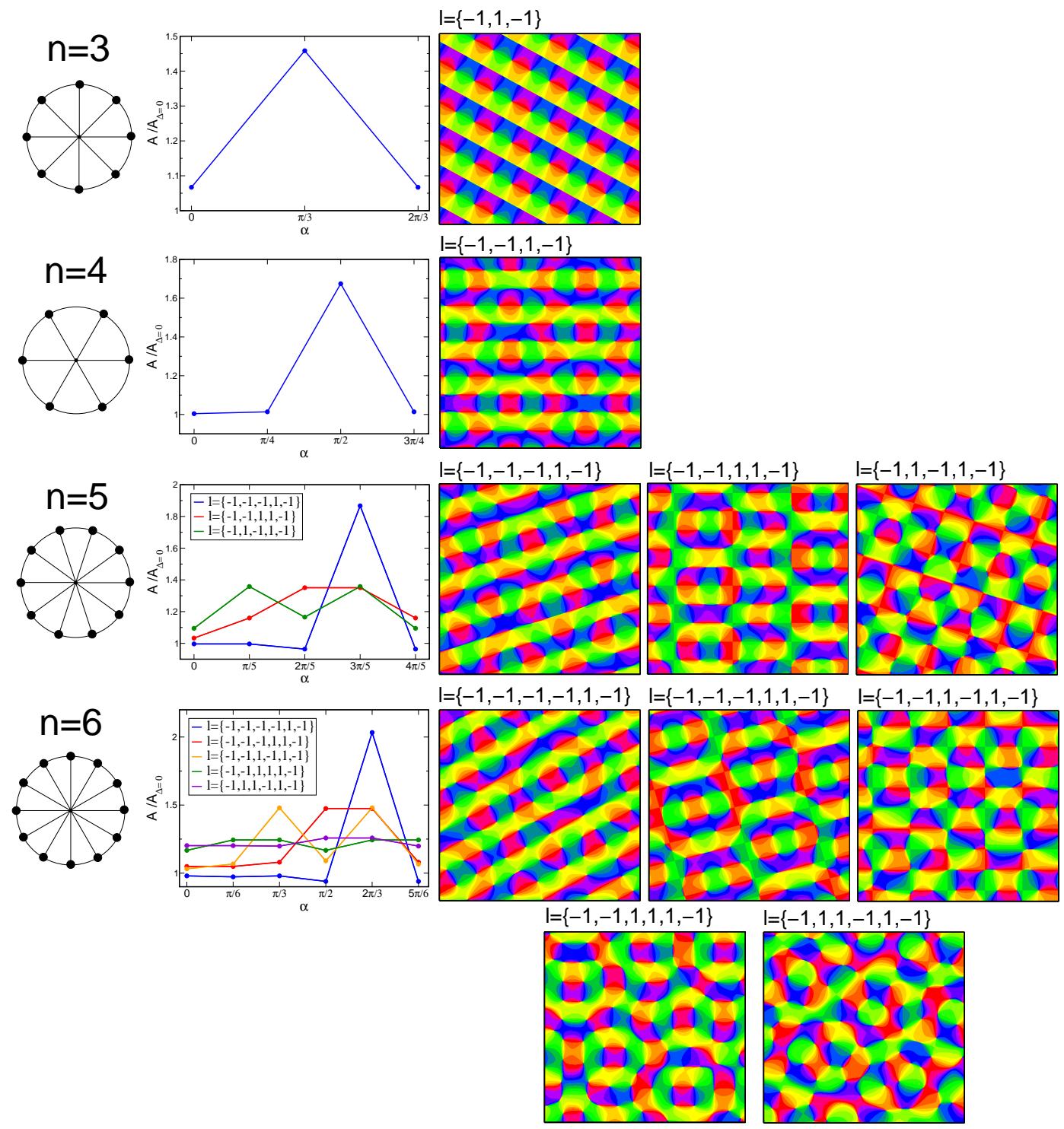

Figure 7.5.: BPP solutions. Left panel: Configuration of active modes. Middle panel: Stationary amplitudes, normalized by the corresponding real $(l=\{-1, \ldots,-1\})$ solution. Right panel: OP map for all non-uniform solutions. Parameters: $g=0.9, \sigma_{2}=\sigma_{1}=0.5 \Lambda(n=3), 0.6 \Lambda(n=4), 0.7 \Lambda(n=5), 0.9 \Lambda(n=6)$. 

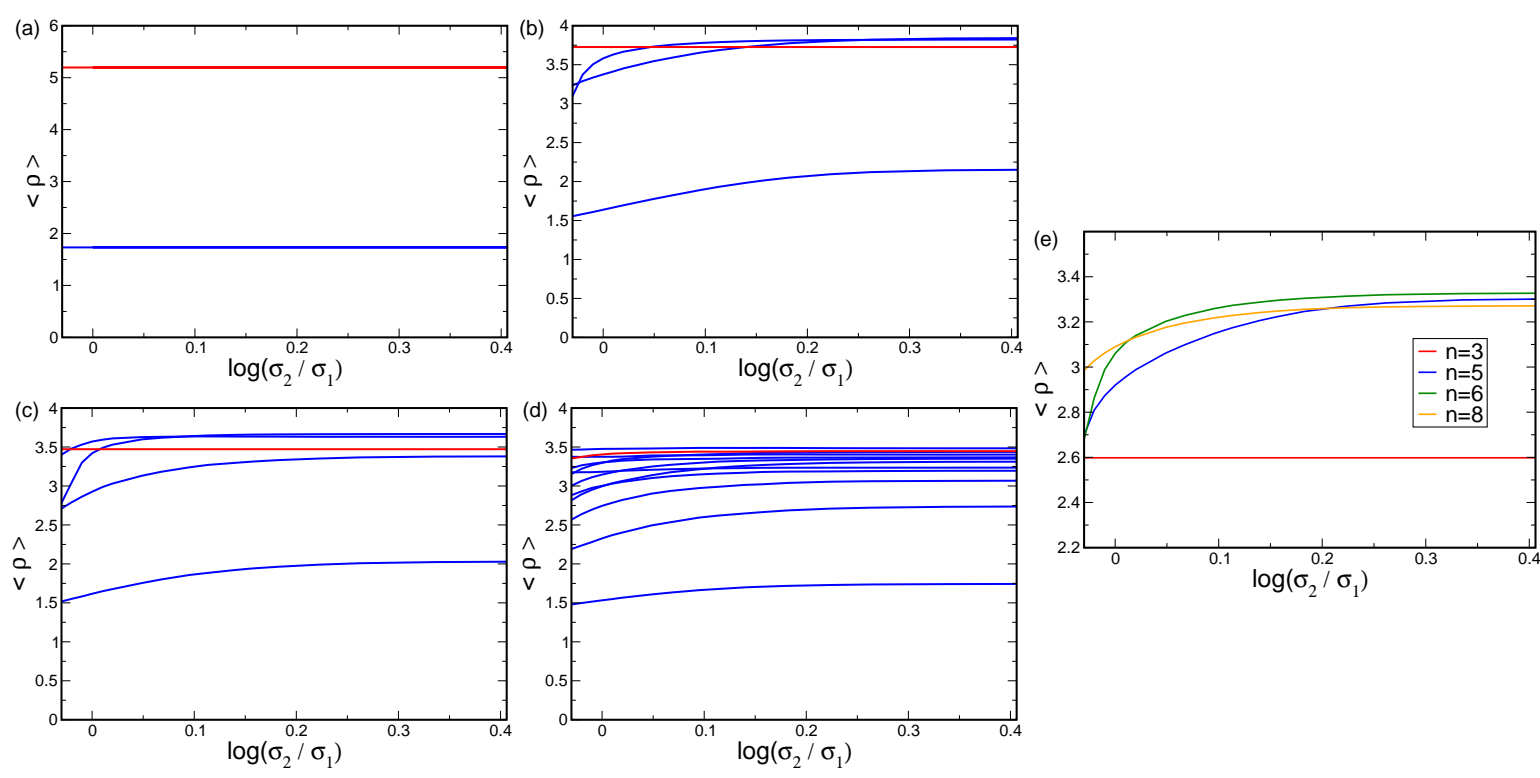

Figure 7.6.: ECP pinwheel densities with broken permutation symmetry. (a) $n=3, \sigma_{1}=0.6 \Lambda$, (b) $n=5, \sigma_{1}=0.8 \Lambda$, (c) $n=6, \sigma_{1}=0.95 \Lambda$, (d) $n=8, \sigma_{1}=1.3 \Lambda$. Red lines: HDP solutions. In all conditions $g=0.98$. (e) Average over random planform realizations, $n=3$ (red), $n=5$ (blue), $n=6$ (green), $n=8$ (orange).

\subsection{Pinwheel densities with broken symmetry}

In this section we study how breaking of permutation symmetry affects the pinwheel density for the different ECP solutions. Furthermore, we study the pinwheel densities of the CPP solutions which are independent of permutation symmetry. Modulations in the stationary amplitudes can lead to a change in the pinwheel densities for the corresponding ECP planforms. Figure [7.6] shows the pinwheel densities for all $n=3,5,6$, and $n=8$ ECP solutions with varying strength of permutation symmetry breaking. HDP solutions are drawn in red. For a fixed $\sigma_{1}$ the range of $\sigma_{2}$ is constrained by stability borders. Parameters $g$ and $\sigma_{1}$ are chosen such that the corresponding $n$ mode solution is intrinsically and extrinsically stable, see Eq. (6.42) and Eq. (6.46). In case of $n=3$ the OP map is a pinwheel crystal and the pinwheel density thus cannot vary continuously. For the considered amplitude modulations the $n=3 \mathrm{LDP}$ solutions receives the pinwheel density stays constant at $\rho=2 \cos \pi / 6$. For $n>3$ the pinwheel density in general depends on permutation symmetry breaking, except for planforms with uniform amplitudes. For $\sigma_{2}<\sigma_{1}$ amplitude modulations in general lead to a decrease in the pinwheel density. For small $\sigma_{2}>\sigma_{1}$ there is a rapid increase in the pinwheel density until at about $\sigma_{2} / \sigma_{1}=1.3$ the pinwheel density saturates. We calculate the average random planform realizations i.e. realizations with randomly chosen $l_{j}$, see Fig. 7.6(e). Compared to the permutation symmetric case, the average pinwheel density increases from $\rho \approx 2.92$ to $\rho \approx 3.3$ for $n=5$, from $\rho \approx 3.06$ to $\rho \approx 3.33$ for $n=6$, and from $\rho \approx 3.09$ to $\rho \approx 3.27$ for $n=8$.

In case of the CPP solution class the pinwheel densities are insensitive to permutation sym- 
metry breaking as their amplitudes are uniform in general. All CPP solutions with $\Delta>0$ have a relatively large pinwheel density, see Fig. 7.4(b). For large $n$ the $\Delta=1$ solution has the largest pinwheel density of $\rho \approx 3.7$ whereas the $\Delta>1$ solutions have a pinwheel density close to $\rho=\pi$.

To summarize, if multistability and the degeneracy of planforms is still preserved with broken permutation symmetry the average pinwheel density substantially increases. But we will see in the next section that the degeneracy is lifted with broken permutation symmetry and that pattern selection can influence the pinwheel density.

\subsection{Lifting the planform degeneracy at leading order}

Does the energetic preference for ECP solutions observed in case of permutation symmetry hold also in case of broken symmetry? What kind of ECP solutions are selected by this symmetry breaking? To answer these questions we calculate the potential Eq. (6.37) at leading order for the ECP and CPP solutions under the influence of permutation symmetry breaking. The potential for the different ECP solutions is shown in Fig. 7.7 (blue lines). Parameters $g$ and $\sigma_{1}$ are chosen such that the corresponding $n$ mode solution is intrinsically and extrinsically stable, see Eq. (6.42) and Eq. (6.46). In the permutation symmetric case the potential is degenerate in the different ECP solutions. With broken symmetry this degeneracy is lifted and planforms are clustered according to their number of flipped mode clusters $\kappa$. For large $\sigma_{1}$ there is even a complete degeneracy of all planforms with a fixed $\kappa$. For $\sigma_{2}>\sigma_{1}$ the HDP solutions, where $\kappa$ is maximal, are the ground state. The energy difference increases with increasing $\sigma_{2}$. In all cases there is a saturation of the energy difference for a large amount of symmetry breaking. In case of $\sigma_{2}<\sigma_{1}$ the LDP solutions $(\kappa=0)$ are energetically selected. Next, we include the potential for the CPP solutions, see Fig. 7.7 (red lines). For every number of active modes $n$ we calculate the potential for all CPP solutions characterized by the integer $\Delta$. Not shown is the potential for the unstable $\Delta=0$ solution which in all cases has a substantially larger potential than that of the remaining CPP and ECP solutions. We observe that the different CPP solutions are energetically not degenerate even in the permutation symmetric case. In case of permutation symmetry all CPP solutions have a substantially larger potential than all the ECP solutions. When permutation symmetry is broken some CPP solutions can have a lower potential than some of the ECP solutions, see Fig. 7.7(d,e). This potential crossing occurs when $\sigma_{1}$ is relatively small and thus the solutions are close to the intrinsic stability border. We identify a single case in which a CPP solution actually becomes the energetic ground state, see Fig. 7.7(a). For broken permutation symmetry the $n=2, \Delta=1 \mathrm{CPP}$ solution is energetically preferred to the $n=1$ and $n=2 \mathrm{ECP}$ solutions. However, for $n>1$ the HDP solutions always correspond to the energetic ground state.

Next, we calculate the phase diagram in case of permutation symmetry. Figure 7.8 shows the regions in the $\left(\sigma_{1} / \Lambda, g\right)$-plane in which certain planforms have minimal energy. We consider all $448 \mathrm{ECP}$ solutions which exist up to $n=13$. In addition, we considered the stable CPP solutions. Figure 7.8(a) corresponds to the permutation symmetric case which is also 
depicted in Fig. [5.1(b). We first study breaking permutation symmetry with $\sigma_{2}>\sigma_{1}$. With increasing $\sigma_{2} / \sigma_{1}$ the effective width of the non-local interactions becomes larger. Therefore, with increasing permutation symmetry breaking there is a shift of all borders in the phase diagram into the left direction. With an increasing amount of permutation symmetry breaking the planforms with an odd number of active modes occupy more and more area in the phase diagram. Whereas in Fig. 7.8(a) all planforms for a given $n$ are degenerate in their energy, the breaking of permutation symmetry selects certain planforms. For $n$ odd the selected planforms are the fully symmetric planforms $l=(1,-1,1,-1, \ldots)$. For $n=4$ the planform $l=(1,-1,1,1)$, for $n=6$ the planform $l=(1,1,-1,1,-1,1)$ and for $n=8$ the planform $l=(1,1,-1,1,-1,1,-1,1)$ is selected. Thus for a given number of active modes the planform with the maximal number of flipped mode clusters $\kappa$ is energetically selected. In the permutation symmetric case CPP solutions never correspond to the energetic ground state. This changes with broken permutation symmetry. With increasing $\sigma_{2} / \sigma_{1}$ the $n=2, \Delta=1 \mathrm{CPP}$ solution has a region (gray) in which it is the energetic ground state. Higher $n$ CPP solutions are nowhere the energetic ground state. Next, we study the change in the phase diagram for $\sigma_{2}<\sigma_{1}$, see Fig. 7.9. Here, with increasing symmetry breaking the effective width of the nonlocal interaction decreases and we thus observe a shift of all stability borders towards higher $\sigma_{1}$ values. In contrast to the case $\sigma_{2}>\sigma_{1}$ the even or odd solutions are not preferred over one another. One exception is the $n=2 \mathrm{ECP}$ solution, which for large symmetry breaking is nowhere the energetic ground state. For the ECP solutions the LDP solutions are always the energetic ground state. Further there is no parameter regime in which the CPP solutions become the energetic ground state.

To summarize, when breaking permutation symmetry such that $\sigma_{2}>\sigma_{1}$, the phase diagram contains only solutions with isotropic mode configurations. When breaking permutation symmetry such that $\sigma_{1}>\sigma_{2}$, only fully anisotropic mode configurations are present in the phase diagram.

\subsection{Stability borders}

We calculate the change in stability properties due to permutation symmetry breaking for all three solution classes using leading order amplitude equations. Intrinsic and extrinsic stability borders are calculated with the use of Eq. (6.42) and Eq. (6.46). The change of the stability borders for all $n=3, n=4$, and $n=5$ ECP solutions is shown in Fig. 7.10, As we use the third order amplitude equations, the stability borders are independent of the bifurcation parameter $r$. In general we observe a shift of all stability borders towards smaller $\sigma_{1}$ values. This change of stability properties is in the symmetry broken case planform dependent. Planforms that are energetically preferred also occupy the largest stability region. Thus in all cases the HDP has the largest stability region. In case of strong symmetry breaking $\left(\sigma_{2}=1.5 \sigma_{1}\right.$, see Fig. 17.10 (c,f,i)) there are values for $g$ in which some planform solution are not stable for any $\sigma_{1}$. In case of $n=3(n=4)$, the HDP solution is the only stable solution below $g \approx 0.55$ $(g \approx 0.63)$. In case of $n=5$ the LDP solution is not stable below $g \approx 0.51$ while the 
$l=(1,-1,1,1,1)$ planform is not stable below $g \approx 0.45$.

We confirmed the intrinsic and extrinsic stability borders by solving the full field dynamics numerically using the stationary amplitudes as initial conditions. To reduce the influence of higher order corrections which are present in the full field dynamics we use a small bifurcation parameter $r=0.01$ and strong permutation symmetry breaking $\sigma_{2} / \sigma_{1}=1.5$. The stability borders obtained by solving the full field dynamics (star symbols in Fig. 7.10 (c,f,i)) are in good agreement with those obtained from amplitude equations. In particular, the LDP solutions become unstable for small $g$. 

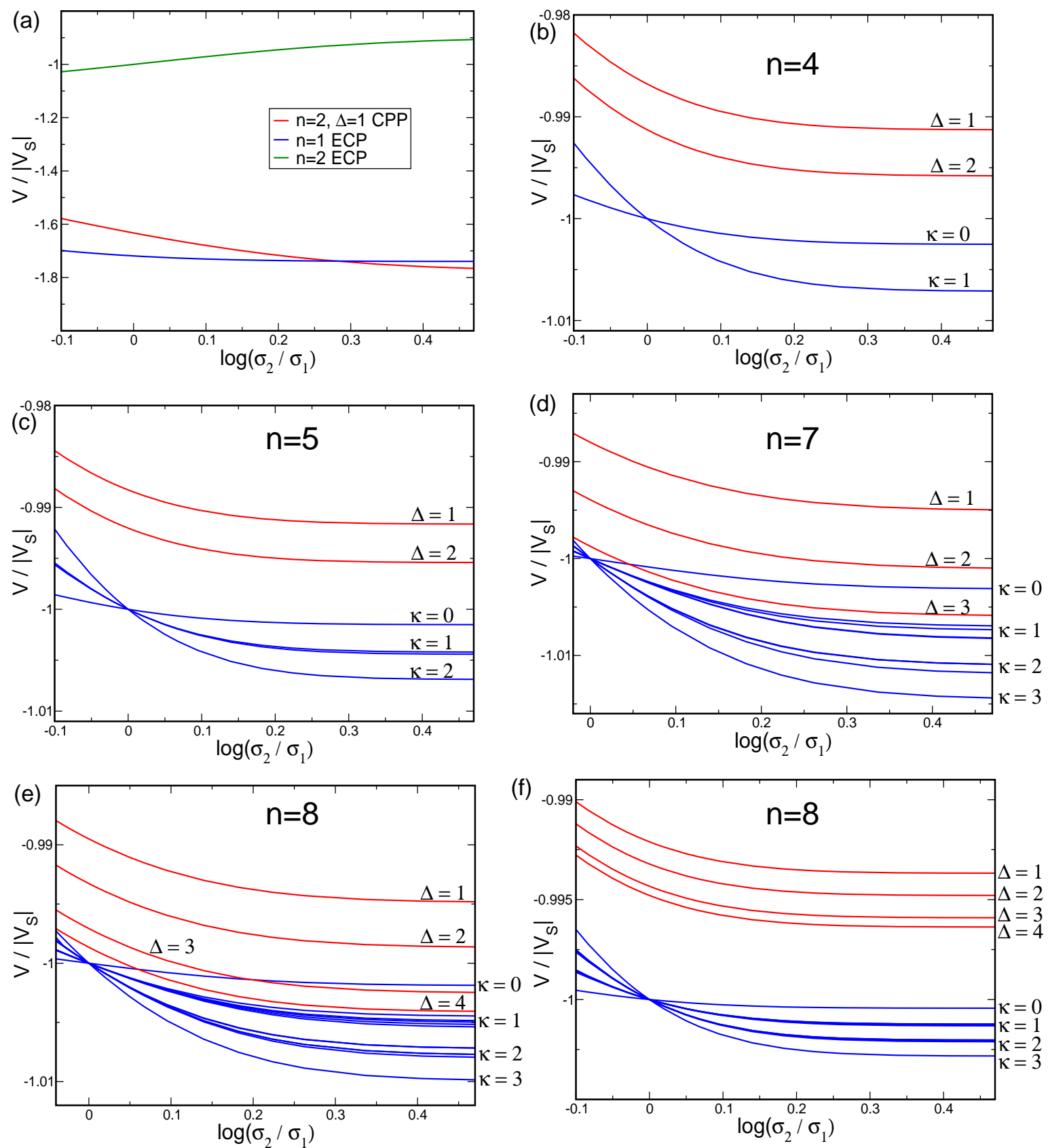

Figure 7.7.: Potential with broken permutation symmetry. Drawn is the potential $V$, normalized by the absolute value of permutation symmetric $\left(\sigma_{2}=\sigma_{1}\right)$ potential $\left|V_{s}\right|$. (a) $\sigma_{1}=0.22 \Lambda$. (b)-(f) ECP solutions (blue), CPP solutions (red). (b) $n=4, \sigma_{1}=0.6 \Lambda$, (c) $n=5, \sigma_{1}=0.75 \Lambda$, (d) $n=7, \sigma_{1}=0.9 \Lambda$, (e) $n=8, \sigma_{1}=1.05 \Lambda$, (f) $n=8, \sigma_{1}=1.23 \Lambda$. In all conditions $g=0.9$. 

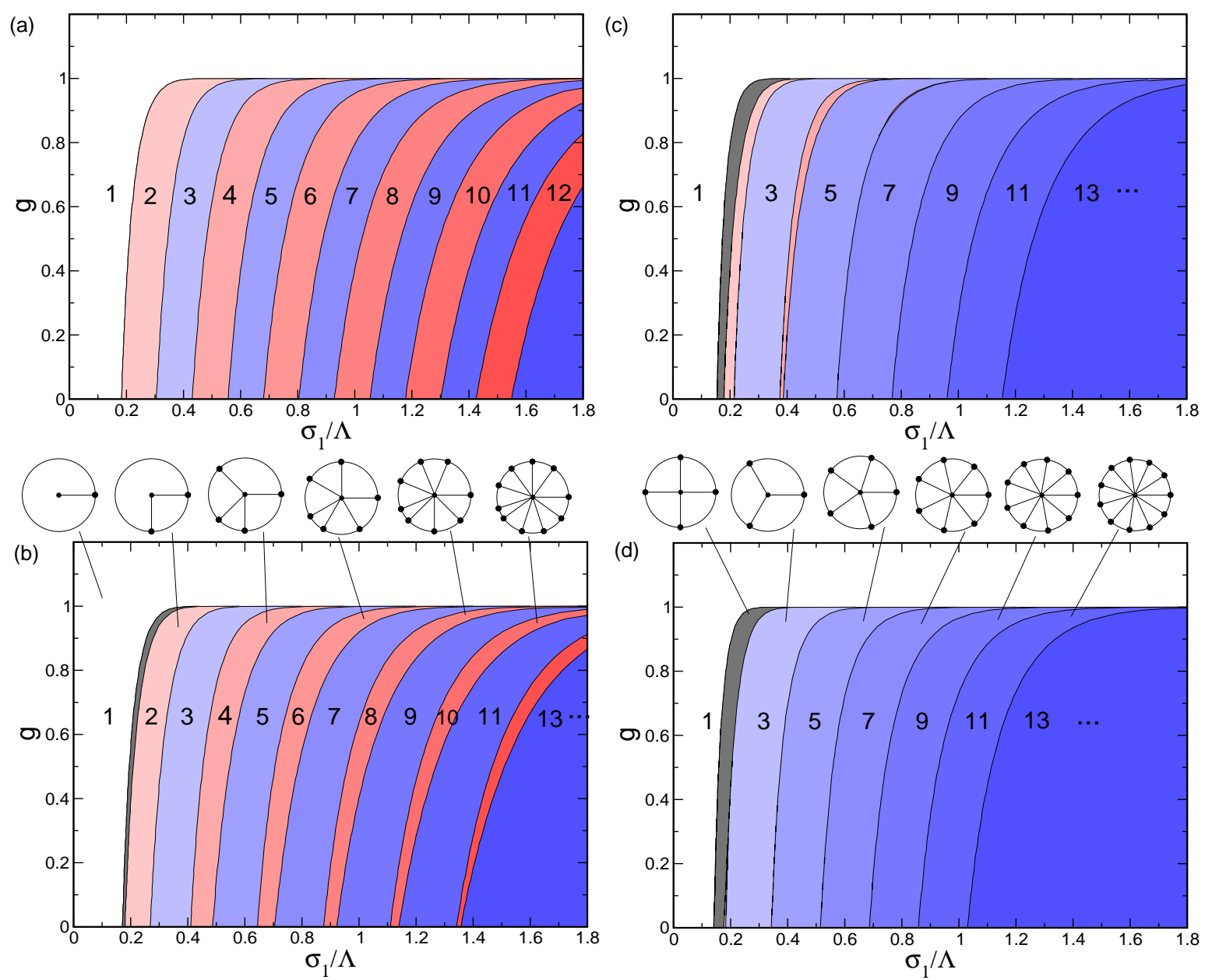

Figure 7.8.: Phase diagram with broken permutation symmetry, $\sigma_{2} \geq \sigma_{1}$. Shown are the regions where a certain planform has the minimal energy, Eq. (6.37). Colored regions: ECP solutions, gray region: $n=2, \Delta=1$ CPP solution. (a) $\sigma_{2} / \sigma_{1}=1$, see also Fig. [5.1(b), (b) $\sigma_{2} / \sigma_{1}=1.1$, (c) $\sigma_{2} / \sigma_{1}=1.3$, (d) $\sigma_{2} / \sigma_{1}=1.5$. 

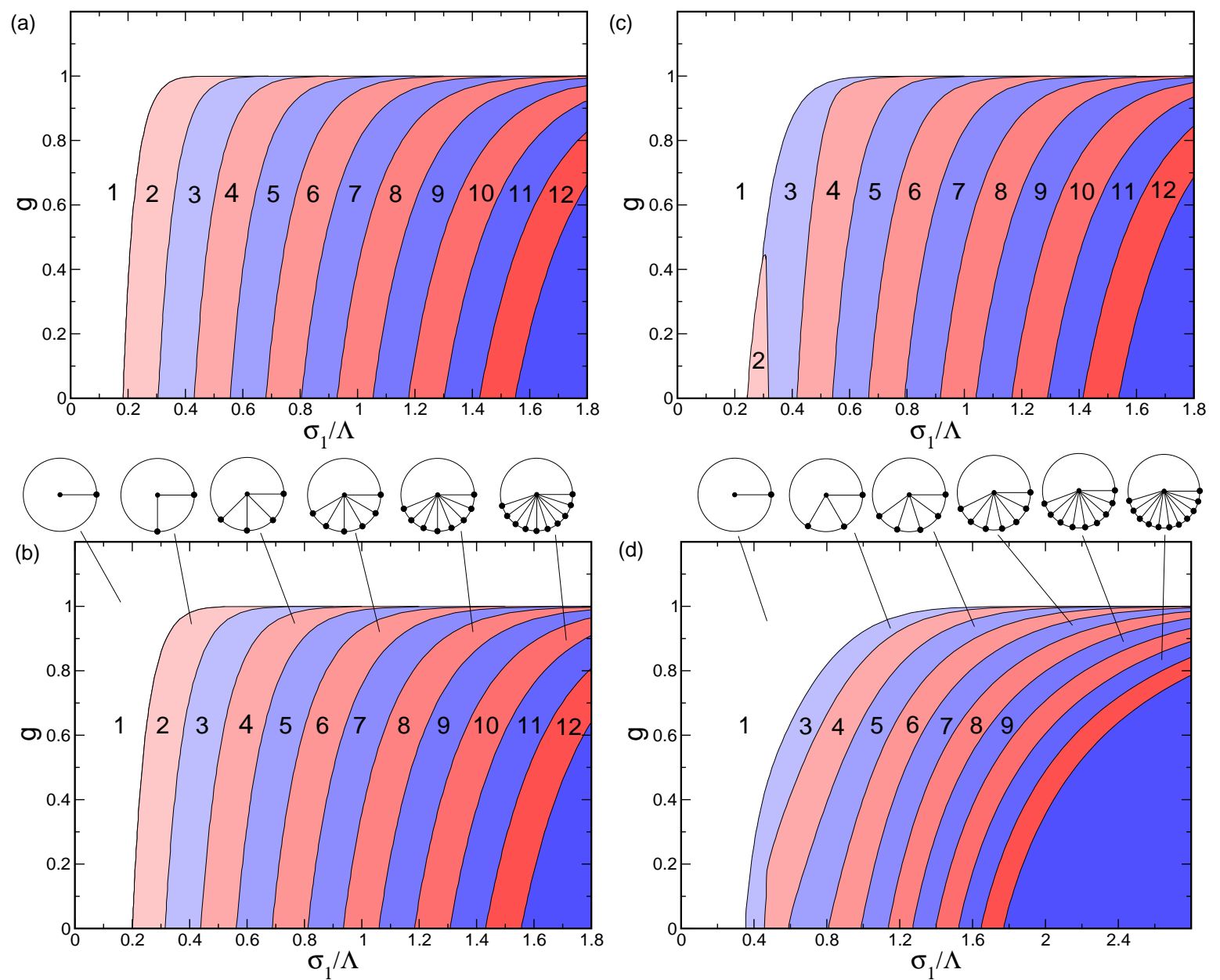

Figure 7.9.: Phase diagram with broken permutation symmetry, $\sigma_{2} \leq \sigma_{1}$. Shown are the regions where a certain planform has the minimal energy, Eq. (6.37). (a) $\sigma_{2} / \sigma_{1}=1$, see also Fig. 5.1(b), (b) $\sigma_{2} / \sigma_{1}=0.8$, (c) $\sigma_{2} / \sigma_{1}=0.5$, (d) $\sigma_{2} / \sigma_{1}=0.2$. 

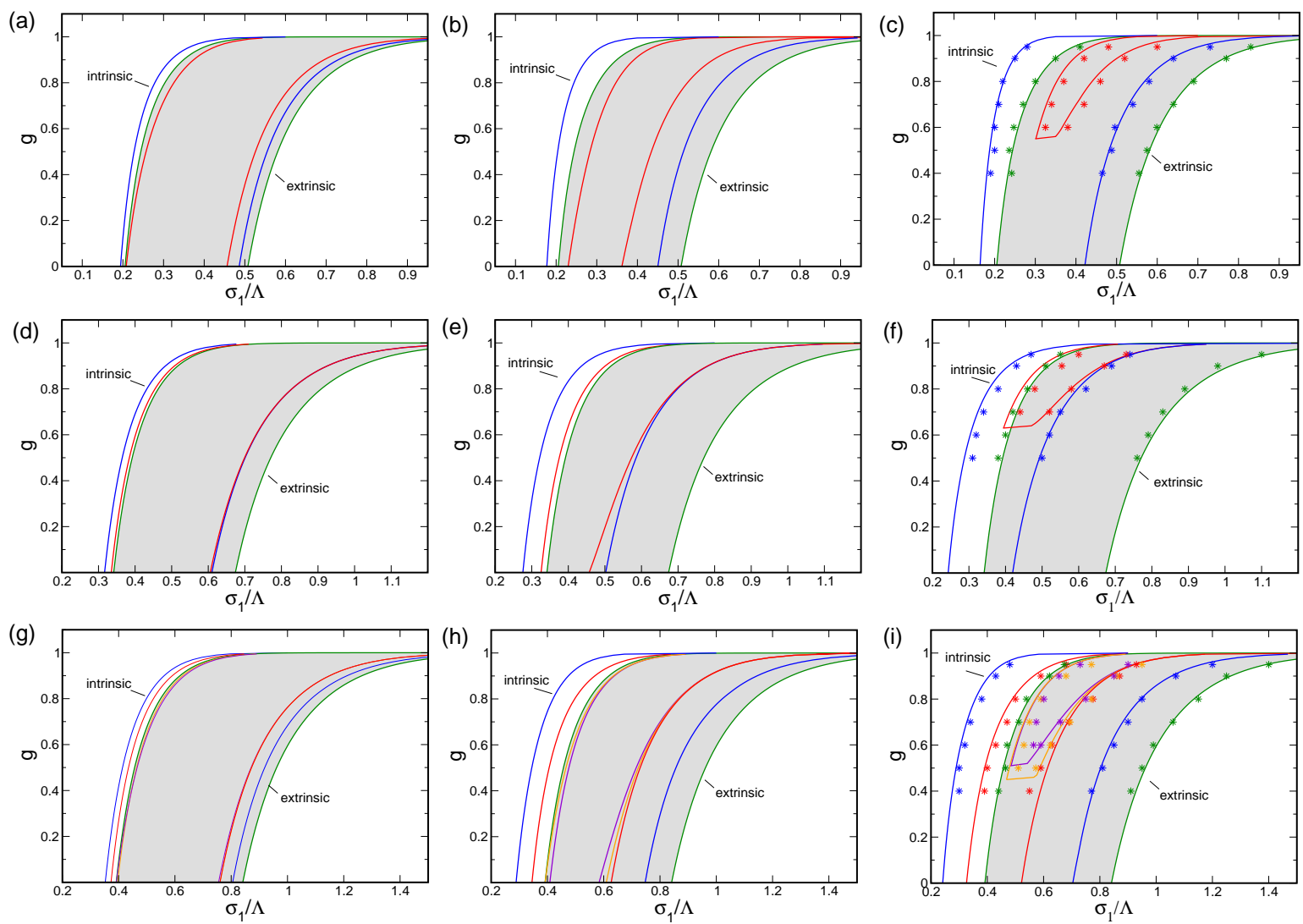

Figure 7.10.: Stability borders of ECP solutions. Borders of intrinsic and extrinsic stability, see Eq. (6.42) and Eq. (6.46). (a)-(c): $n=3$ with $l=(1,1,1)$ (red) and $l=(1,-1,1)$ (blue). Green: permutation symmetric model. (d)-(f): $n=4$ with $l=(1,1,1,1)$ (red), $l=(1,0,1,1)$ (blue). (g)-(i): $n=5$ with $l=(1,1,1,1,1)$ (red), $l=(1,-1,1,1,1)$ (violet), $l=(1,1,-1,1,1)$ (orange), and $l=(1,-1,1,1,-1,1)$ (blue). $(\mathbf{a}, \mathbf{d}, \mathbf{g}) \sigma_{2} / \sigma_{1}=1.1,(\mathbf{b}, \mathbf{e}, \mathbf{h}) \sigma_{2} / \sigma_{1}=1.3,(\mathbf{c}, \mathbf{f}, \mathbf{i}) \sigma_{2} / \sigma_{1}=1.5$. Star symbols: Stability borders from the full field dynamics. Parameters: $r=0.01, \Gamma=40, T_{f}=5 \cdot 10^{4}, 256 \times 256$ mesh. 

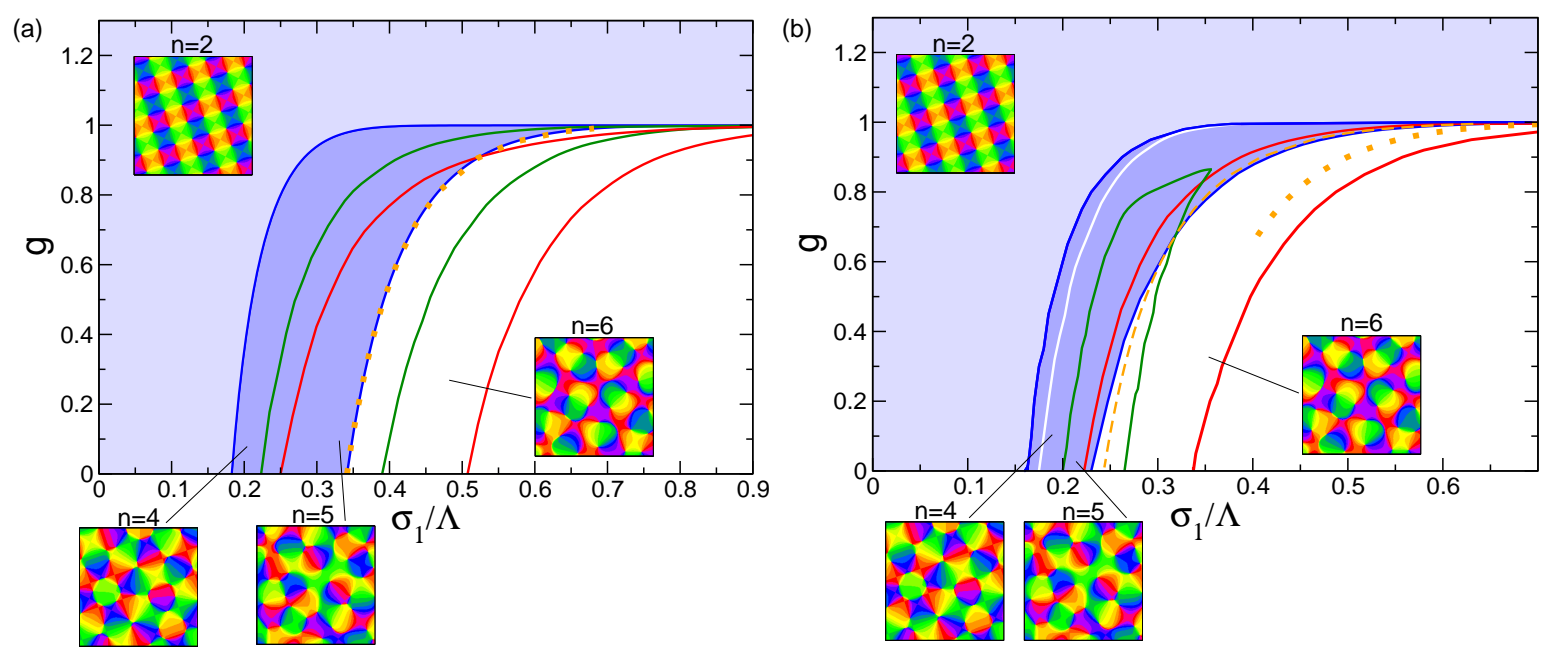

Figure 7.11.: Stability borders of CPP solutions. (a) $\sigma_{2}=\sigma_{1}$, (b) $\sigma_{2}=1.5 \sigma_{1}$. Light blue region: $n=2, \Delta=1$ solution stable, dark blue region: $n=4, \Delta=2$ solution stable, green lines: stability range of $n=5, \Delta=2$ solution, red lines: stability range of $n=6, \Delta=3$ solution, white line: stability line of $n=2, \Delta=1$ solution. Dashed (dotted) orange line: Intrinsic stability border of $n=4 \mathrm{HDP}$ (LDP) solution.

Next, we consider the stability properties of the CPP solutions for which $\Delta>0$. In this case we have to consider amplitude $\mathcal{A}_{j}=\mathcal{A}+a_{j}$ and phase $\Phi_{j}=\Phi_{j}^{*}+\varphi_{j}$ perturbations which do not necessarily decouple. We analyze the stability properties for all uniform solutions which exist for a given number of active modes. The stability regions of the $n=2, \ldots, 6 \mathrm{CPP}$ solutions is shown in Fig. 7.11. The $n=2, \Delta=1$ solution is stable for $g>1$ or $\sigma_{1}$ small. For $n=3$ it turns out that none of the uniform solutions are stable. For $n \geq 4$ the shape of the stability regions resemble those of the stability regions for the ECP solutions. For $g>1$ and with increasing $\sigma_{1} / \Lambda$ higher $n$ solutions become stable. Compared to the permutation symmetric case the stability borders in case of broken symmetry $\left(\sigma_{2}=1.5 \sigma_{1}\right)$ are shifted towards smaller $\sigma_{1}$ values. In contrast to the ECP solutions there is no multistability of the CPP solutions for a fixed $n$, even in the permutation symmetric case. Thus for a given $n$ there is at maximum one solution which can become stable. However, there can be a multistability of CPP solutions with different $n$. For instance, the $n=4, \Delta=2$ and the $n=5, \Delta=2$ solutions have partly overlapping stability regions.

How do the stability borders of ECP and CPP solutions relate to each other? When we compare the stability borders of the ECP and CPP solutions we observe that in the permutation symmetric case the stability borders of the $n$ mode CPP solutions coincide with the intrinsic stability borders of the $n$ mode ECP solution. This is illustrated in case of $n=4$ in Fig. 7.11(a). Therefore in the permutation symmetric case $n$ mode ECP and CPP solutions have no overlapping region of stability. In case of broken permutation symmetry the stability borders of ECP and CPP do not coincide but the $n$ modes CPP stability border is close to the intrinsic border of the $n$ modes HDP solutions, see Fig. 7.11(b).

As for the ECP and CPP solutions we study the stability properties of the BPP solutions in 

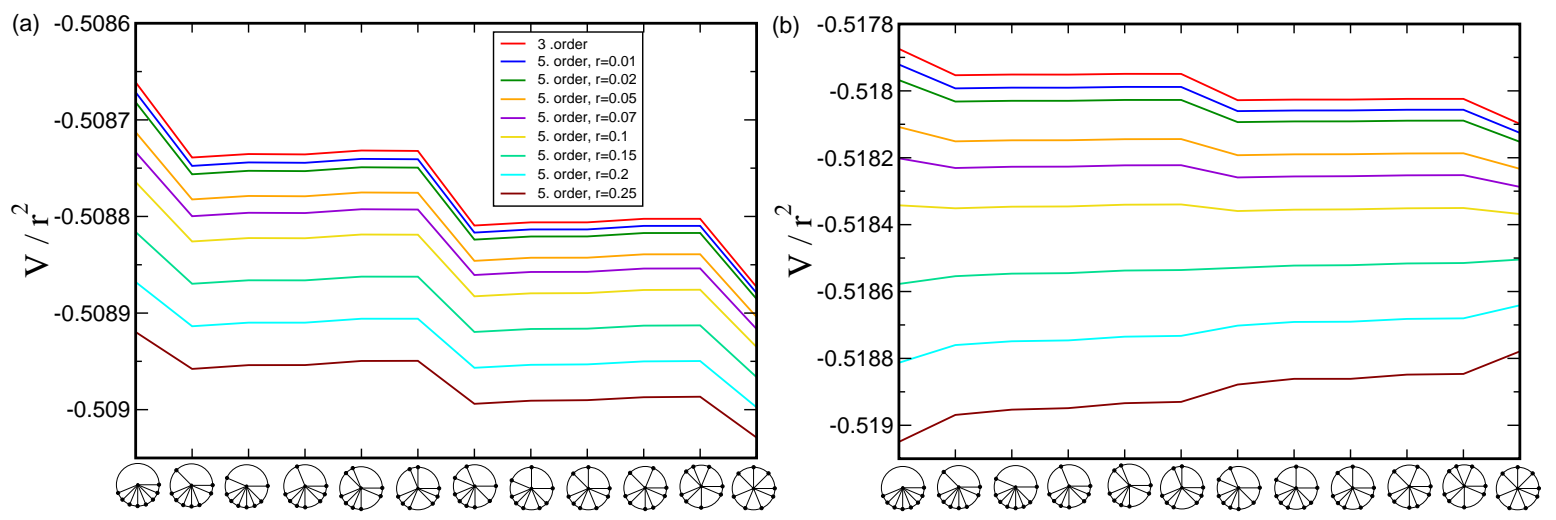

Figure 7.12.: Interplay between third and fifth order planform selection. Potential $V$, Eq. (6.37), in the permutation symmetry broken case with increasing distance from threshold for all $n=8$ ECP solutions. (a) $g=0.98$, (b) $g=0.96$. In all conditions: $\sigma_{1}=1.43 \Lambda, \sigma_{2}=1.5 \sigma_{1}$.

the presence and absence of permutation symmetry. We calculate stationary amplitudes of Eq. (7.12) and corresponding stability matrices numerically in the same parameter region as we did for the ECP and CPP solutions. It turns out, however, that none of the non-uniform solutions are stable for $g>0, \sigma_{1}, \sigma_{2}>0$.

\subsection{Including higher order corrections}

To reveal the interplay between permutation symmetry breaking, affecting pattern selection at third order, and fifth order corrections, discussed in Chapter [6, we compared the potential Eq. (6.37) of the planforms with increasing influence of the fifth order corrections measured in $r$, see Fig. 7.12, We observe that depending on the influence of fifth order corrections high pinwheel density planforms or low pinwheel density planforms are selected. The influence of fifth order corrections increases as $(1-g)^{2}$, thus HDP solutions are selected only for $g$ close to 1 . For the chosen parameter values $g=0.98$ and $g=0.96$ the planforms can be clustered according to their number of flipped modes clusters $\kappa$. For $g=0.98$ the order of planforms obtained at third order is preserved also far from threshold i.e. HDP solutions are energetically preferred. The strong dependence on the parameter $g$ becomes apparent we calculate the potential for $g=0.96$. Here, with increasing $r$, the ordering of the planforms is reversed and for $r>0.1$ the LDP solution is the energetic ground state. These results show that even when considering higher order corrections there is a parameter regime close to threshold in which the HDP solutions are energetically preferred.

\subsection{Numerical analysis of pattern selection}

We confirm the results obtained from weakly nonlinear analysis by solving the full field dynamics Eq. (6.1) with the nonlinearities Eq. (7.9) numerically. To reveal the interplay between the influence of permutation symmetry breaking and of higher order corrections to the am- 
plitude equations we simulate the field dynamics close to threshold $(r=0.01)$ and further away from threshold $(r=0.2)$. For a fixed set of $N=20$ band-pass filtered Gaussian random fields as initial conditions we calculated the time evolution of the pinwheel density in the presence and absence of permutation symmetry. We break permutation symmetry in both directions. When choosing $\sigma_{2}=1.5 \sigma_{1}$ previous results from amplitude equations suggest that HDP are energetically selected and the average pinwheel density should increase compared to the permutation symmetric case. When choosing $\sigma_{2}=0.5 \sigma_{1}$, LDP solutions are energetically selected and thus the average pinwheel density should decrease. The results for simulations close to threshold are shown in Fig. 7.13. The pinwheel density evolves in all three cases ( $\sigma_{2}=\sigma_{1}$ red, $\sigma_{2}=1.5 \sigma_{1}$ blue, $\sigma_{2}=0.5 \sigma_{1}$ green) similar up to times $T \approx 1$ and the average pinwheel density is almost identical. At about $T=1$ there is a rapid increase in the power, see Fig. 17.13(d) until a saturation takes place at $T \approx 10$ with $P\left(T_{f}\right)=n r / \sum_{j} g_{i j}$. From thereon the pinwheel densities in the symmetry broken case are slightly higher $\left(\sigma_{2}>\sigma_{1}\right)$ or lower $\left(\sigma_{2}<\sigma_{1}\right)$ than in the symmetric case. Between $T \approx 10^{2}$ and $T \approx 10^{4}$ the final patterns are selected. Whereas in the permutation symmetric case there is a rather broad distribution of pinwheel densities between $\rho \approx 1.5$ and $\rho \approx 3.5$, permutation symmetry breaking selects certain planform solutions and the distribution of pinwheel densities is more narrow, see Fig. 7.13 (c). The average pinwheel densities in the final states are $\langle\rho\rangle \approx 3$ in the permutation symmetric case, $\langle\rho\rangle \approx 3.35$ for $\sigma_{2}=1.5 \sigma_{1}$, and $\langle\rho\rangle \approx 1.38$ for $\sigma_{2}=0.5 \sigma_{1}$, see Fig. 7.13(b). Thus there is a small increase of the average pinwheel density for $\sigma_{2}=1.5 \sigma_{1}$ and a larger decrease for $\sigma_{2}=0.5 \sigma_{1}$. In the permutation symmetric case the final pattern mainly consist of $n=7$ and $n=8 \mathrm{ECP}$ solutions $\left(\sigma_{1}=1.4 \Lambda\right)$. In contrast, in the case of $\sigma_{2}=1.5 \sigma_{1}$, the final patterns mainly consist of $n=9$ and $n=10 \mathrm{HDP}$ solutions. LDP solutions were completely absent in this case. In case of $\sigma_{2}=0.5 \sigma_{1}$, except a single simulation resulting in a $n=5$ planform with a very high pinwheel density of $\rho \approx 3.8$, the final patterns mainly consist of $n=5 \mathrm{LDP}$ solutions. We further calculated the time dependent cross correlation between the fields in the permutation symmetric and the symmetry broken case, i.e.

$$
C(t)=\operatorname{Re} \int z_{1}(\mathbf{x}, t) \bar{z}_{2}(\mathbf{x}, t) d^{2} x /\left(\int\left|z_{1}(\mathbf{x}, t)\right|^{2} d^{2} x \int\left|z_{2}(\mathbf{x}, t)\right|^{2} d^{2} x\right)
$$

where $z_{1}$ and $z_{2}$ are the OP maps in the permutation symmetric and symmetry broken case, respectively. Correlations decrease as the power $P$ is saturated, see Fig. 7.13)(d). In case of $\sigma_{2}=0.5 \sigma_{1}$ the patterns are less correlated than in the case of $\sigma_{2}=1.5 \sigma_{1}$. A reason for this might be the fact that in case of $\sigma_{2}=0.5 \sigma_{1}$ more active modes are flipped when permutation symmetry is broken.

Next, we study pattern selection far from threshold, see Fig. 7.14. Here, the average pinwheel density in the permutation symmetric case is smaller $\langle\rho\rangle \approx 2.38$ than it is close to threshold. As discussed in the previous chapter we can explain this by higher order corrections to the amplitude equations which select LDP solutions. The pinwheel density evolves similar in all three cases until $T \approx 1$. Between $T \approx 1$ and $T \approx 10$ the modes saturate and the power $P(t)$ is approximately at its final value. From thereon the pinwheel density in the symmetry broken 
case is slightly higher/lower than that in the symmetric case. Between $T \approx 10^{3}$ and $T \approx 10^{4}$ the different planform solutions are selected. Again, breaking permutation symmetry leads to a narrow distribution of final pinwheel densities, see Fig. 7.14(c) The final pinwheel densities are $\langle\rho\rangle=3.28$ for $\sigma_{2}=1.5 \sigma_{1},\langle\rho\rangle=2.38$ for $\sigma_{2}=\sigma_{1}$, and $\langle\rho\rangle=1.12$ for $\sigma_{2}=0.5 \sigma_{1}$, see Fig. 7.14(b). In these simulations low density planforms are absent for $\sigma_{2}>\sigma_{1}$.

(a)

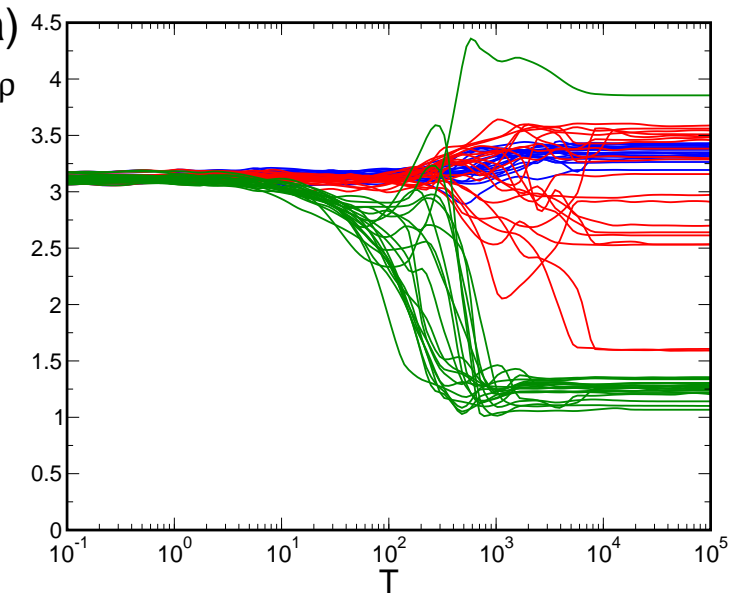

(b)

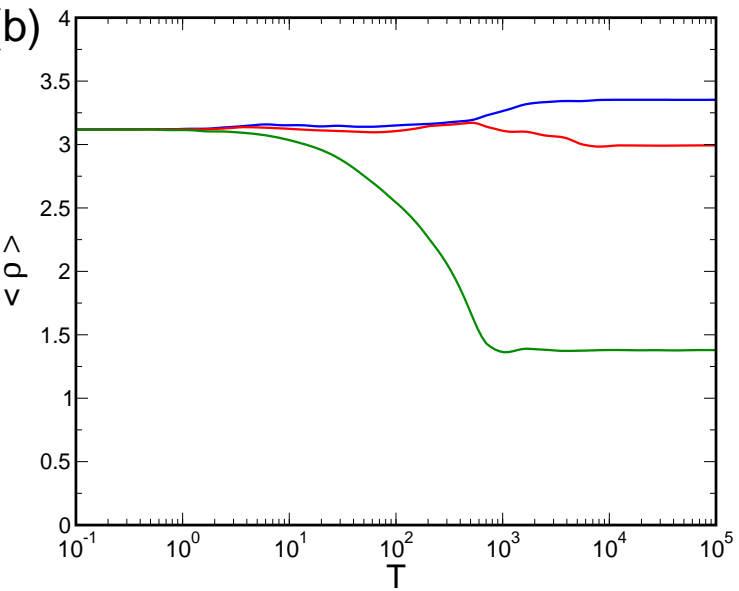

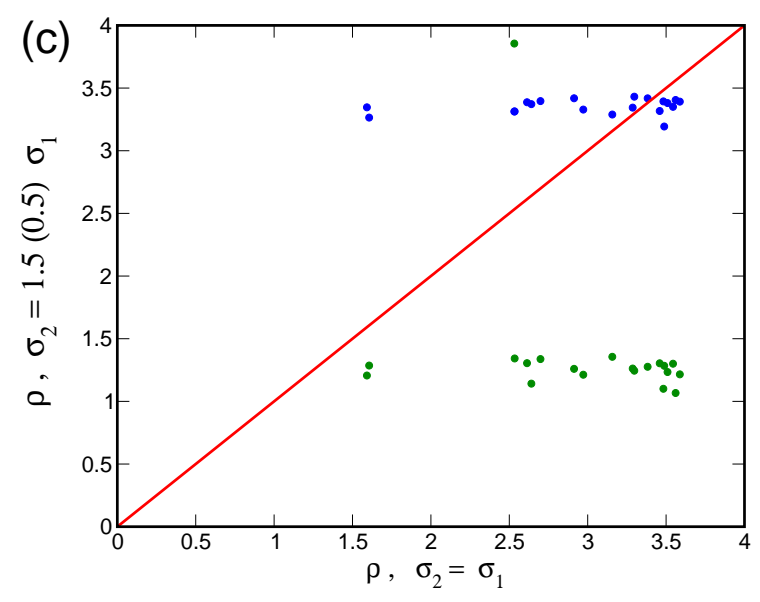

(d)

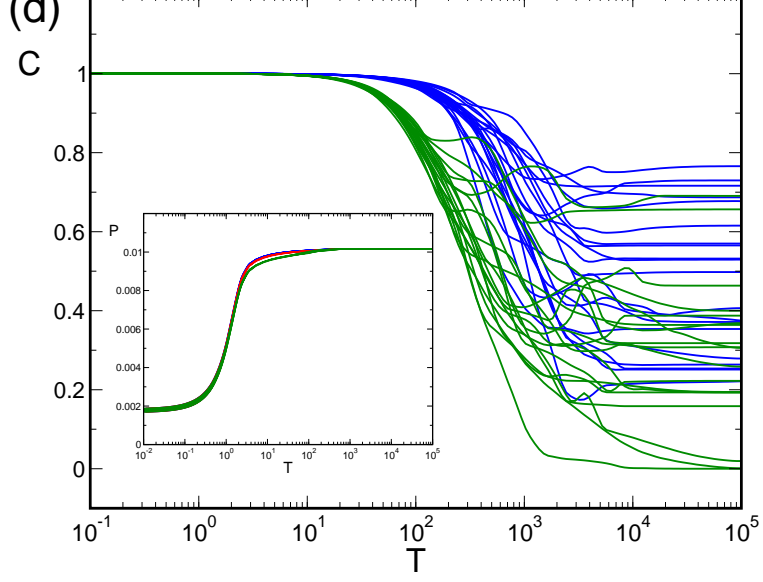

Figure 7.13.: Time evolution of the pinwheel density near threshold. (a) Pinwheel densities for 20 initial conditions. Red lines: Permutation symmetric model, blue lines: the broken symmetry model Eq. (7.9) with $\sigma_{2}=1.5 \sigma_{1}$, green lines: $\sigma_{2}=0.5 \sigma_{1}$. (b) Mean pinwheel densities in the symmetry broken and unbroken case. (c) Comparison of pinwheel densities for the final states, $T_{f}=10^{5}$. (d) Cross correlation between permutation symmetric and symmetry broken $\left(\sigma_{2}=1.5 \sigma_{1}\right.$ blue, $\sigma_{2}=0.5 \sigma_{1}$ green $)$ maps. Inset: Time evolution of the Power $P(t)$. For all simulations: $r=0.01, g=0.98, \sigma_{1}=1.43 \Lambda, \Gamma=40$, $256 \times 256$ mesh. 
(a)

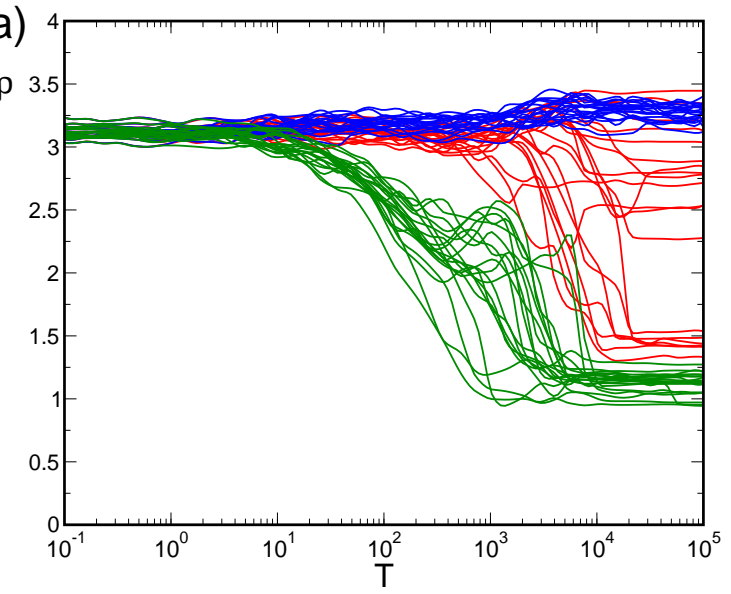

(b)

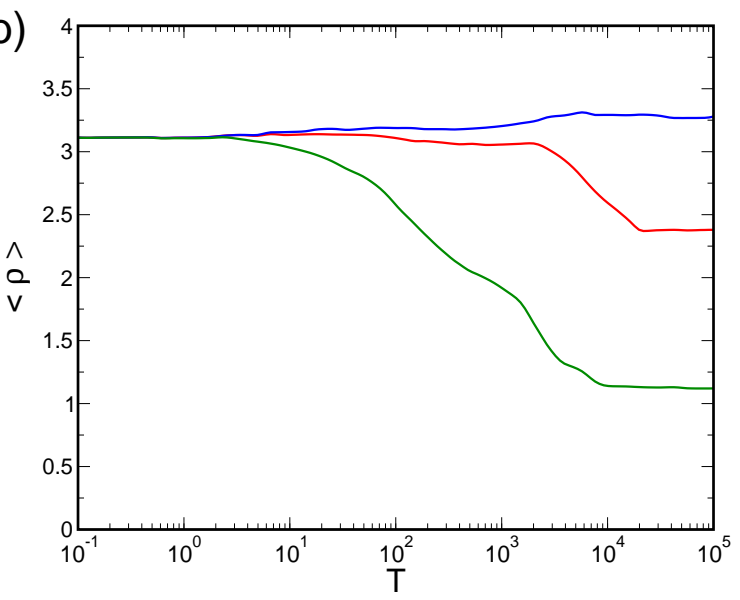

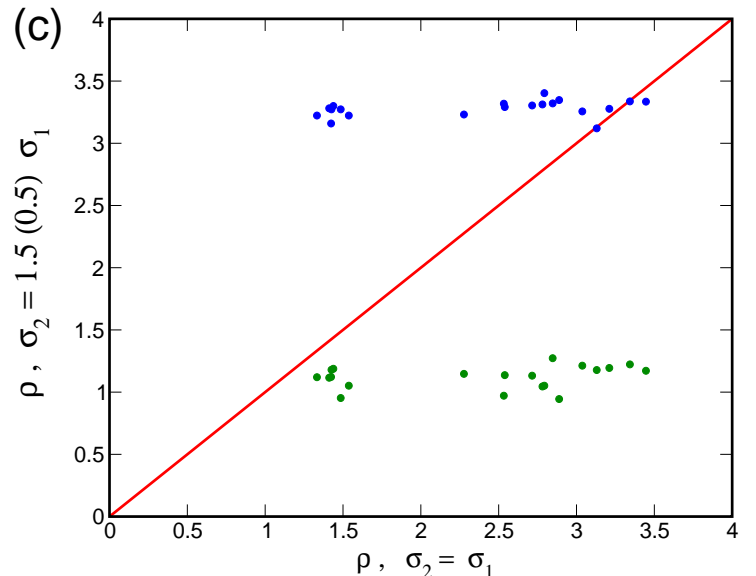

(d)

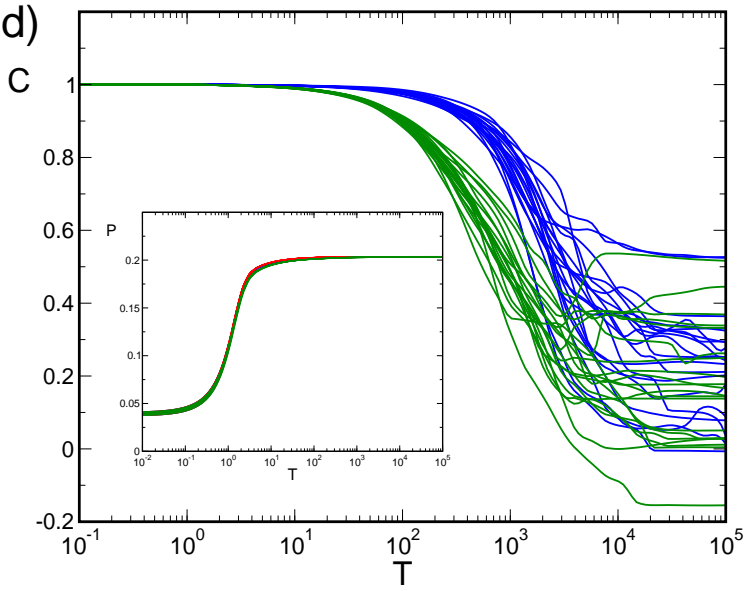

Figure 7.14.: Time evolution of the pinwheel density far from threshold. (a) Pinwheel densities for 20 initial conditions. Red lines: Permutation symmetric model, blue lines: the broken symmetry model Eq. (17.9) with $\sigma_{2}=1.5 \sigma_{1}$, green lines: $\sigma_{2}=0.5 \sigma_{1}$. (b) Mean pinwheel densities in the symmetry broken and unbroken case. (c) Comparison of pinwheel densities for the final states, $T_{f}=10^{5}$. (d) Cross correlation between permutation symmetric and symmetry broken $\left(\sigma_{2}=1.5 \sigma_{1}\right.$ blue, $\sigma_{2}=0.5 \sigma_{1}$ green $)$ maps. Inset: Time evolution of the Power $P(t)$. For all simulations: $r=0.2, g=0.98, \sigma_{1}=1.43 \Lambda, \Gamma=40$, $256 \times 256$ mesh. 

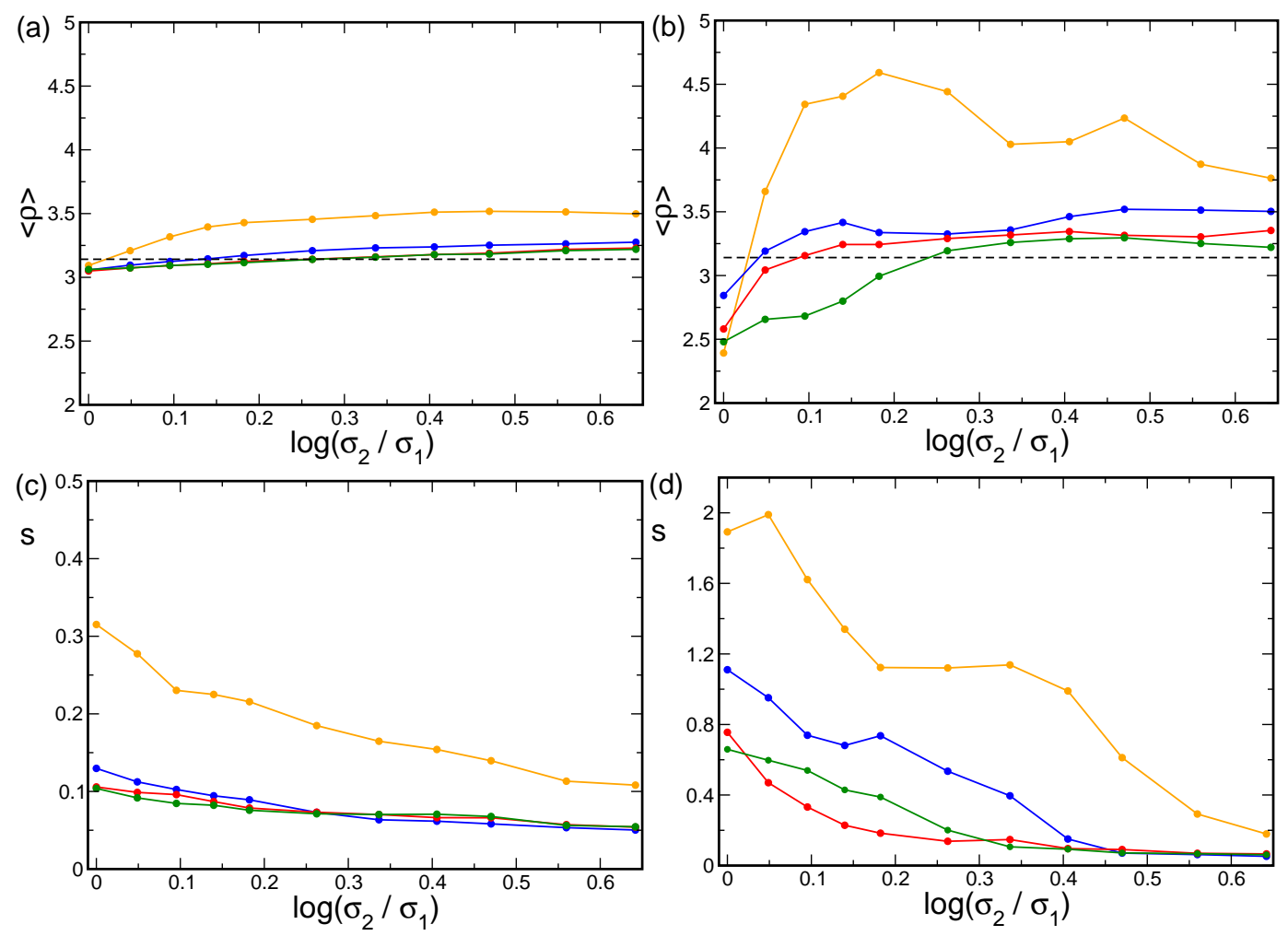

Figure 7.15.: Raising pinwheel densities by pattern selection. (a,b) Change in average pinwheel density due to permutation symmetry breaking, orange: $\sigma_{1}=0.54 \Lambda$, blue: $\sigma_{1}=0.89 \Lambda$, red: $\sigma_{1}=1.43 \Lambda$, green: $\sigma_{1}=1.79 \Lambda$. Dashed line: $\rho=\pi$. (c,d) Standard deviation of pinwheel densities. (a,c) $T_{f}=300$, (b,d) $T_{f}=10^{5}$. In all simulations: $g=0.98, r=0.2, \Gamma=40,256 \times 256$ mesh.

Next, we study the gradual change in the pinwheel density with increasing amount of symmetry breaking. For a fixed set of 50 initial conditions we varied the amount of symmetry breaking $\sigma_{2} / \sigma_{1}$, see Fig. 7.15, In the symmetric case the theoretical value for the pinwheel density near threshold is $\rho=\pi$. Deviations from this value can be explained by higher order corrections which increase with increasing $\sigma$, see also Fig. 6.6. For $\sigma_{1}=0.54 \Lambda$ (orange line) stationary solutions in the permutation symmetric case are $n=2$ and $n=3$ ECP solutions. The resulting average pinwheel density is thus rather low $(\langle\rho\rangle \approx 2)$. With increasing permutation symmetry breaking $n=3 \mathrm{HDP}$ solutions show up more frequently and the pinwheel density increases up to $\langle\rho\rangle \approx 4.7$. Increasing permutation symmetry breaking even more $n=4$ and $n=5$ HDP solutions occur and the average pinwheel density decreases but is still large. Note, that the set of stationary solutions contains some $n=4, \Delta=2 \mathrm{CPP}$ solutions. For very large symmetry breaking the $n=5 \mathrm{HDP}$ solutions are most dominant and the average pinwheel density is about $\langle\rho\rangle \approx 3.75$. For small symmetry breaking the average pinwheel density rapidly increases with increasing $\sigma_{2} / \sigma_{1}$ until a saturation of the pinwheel density is achieved. A further increase of $\sigma_{2} / \sigma_{1}$ leads at some point to an extrinsic instability of the current solution and to stable higher mode solutions. Figure 7.15(b) shows the standard deviation $s$ of the pinwheel densities. As the symmetry breaking selects certain planform 
solutions the standard deviation decreases with increasing $\sigma_{2} / \sigma_{1}$.

To summarize, the numerical simulations confirmed the predictions obtained from amplitude equations that breaking permutation symmetry selects either low or high pinwheel density planforms. The average pinwheel density, depending on the direction of symmetry breaking, increases or decreases and the distribution of pinwheel densities becomes narrow. The results demonstrate that even far from threshold solutions with a relatively large pinwheel density can be selected.

\subsection{Discussion}

In this chapter we studied pattern selection by breaking the permutation symmetry of the OP dynamics. Compared to rotation, translation, or orientation shift symmetry permutation symmetry has no clear biological interpretation. In previous models for the development of OP maps, like the elastic net model, the resulting cubic nonlinearities are in general not permutation symmetric [119]. To compare with such models and to provide a general theory of OP map development with translation, rotation and orientation shift symmetry we considered permutation symmetry breaking. There are several ways to break permutation symmetry in our model but we have shown that not all of them lead to biological meaningful OP maps. We broke this symmetry introducing a disbalance between the two non-local interaction terms, allowing for two typical length-scales for these interactions. Compared to a Swift-Hohenberg equation with a real order parameter field, see for instance [4, 97, 120], its complex pendant shows an enormous variety of stationary solutions. In the permutation symmetric case the ECP class is the dominant solution class with a large number of multistable solutions. Remarkably, the number of potential solutions becomes even larger in case of broken permutation symmetry as additional solution classes can become important.

We first studied the impact of permutation symmetry breaking on ECP solutions. These solutions are model independent in the case of permutation symmetry but become model dependent in the symmetry broken case as their stationary amplitudes are modulated. We demonstrated how such modulations can change the pinwheel density of the corresponding ECP solution. Moreover, permutation symmetry breaking energetically selects certain ECP solutions. We showed that, in contrast to the previous chapter, HDP solutions can be energetically selected. Breaking permutation symmetry thus can lead to realistic pinwheel densities even far from threshold. Moreover, breaking permutation symmetry affects the stability borders of ECP solutions. We showed that ECP solutions which are energetically preferred also have the largest region of stability. However, even for strong permutation symmetry breaking multistability is not completely lifted. A potential switching between different multistable solutions, as indicated by experiments [16, 88, is therefore not in contradiction with broken permutation symmetry.

Next, we considered CPP solutions for which each Fourier mode is accompanied by a mode in the opposite direction. This solution class contains solutions which are selective to only two preferred orientations, a type of solution that occurs in the real Swift-Hohenberg equations. 
We therefore showed that, although these orientation scotoma solutions can become stable for a general permutation symmetry broken model, they are unstable in our model. CPP solutions do not play a role in the case of permutation symmetry. In contrast, when permutation symmetry is broken, solutions within this class can become the energetic ground state of the model. The CPP solutions have uniform stationary amplitudes both in the presence and absence of permutation symmetry, and this solution class is thus model independent in general. Finally, we considered BPP solutions for which stationary amplitudes are not uniform and therefore model dependent in general. We showed, however, that this class of solutions is in general unstable in our model. To summarize, we identified three main causes for a change in the average pinwheel density compared to the permutation symmetric model: Modulations in the ECP amplitudes, energetic selection of a particular ECP solution, and the occurrence of the CPP solution class with comparable energies to the ECP solutions.

For a general theory of visual cortical development, all three classes of stationary solutions can become important. As the CPP solution class is in general model independent and contains solutions with a pinwheel density close to $\rho=\pi$ it would be instructive to construct a model in which these solutions are dominant. As we have seen in this chapter breaking permutation symmetry can be a first step in this direction. 


\section{Conclusion}

In this thesis we studied pattern formation in models of visual cortical development. The development of cortical maps has been conventionally treated with dimension reduction models which, as a numerical approach, often fail to produce realistic maps. Instead of proposing a specific optimization principle we presented a model independent approach to analyze the simultaneous development of different visual cortical maps. In addition we could treat map interactions analytically. The presented approach is largely based on symmetry principles. Symmetries are an important aspect of numerous physical disciplines, they range from the fundamental equations in particle physics which are governed by gauge symmetries up to the large scales in astronomy governed by the space time symmetries of general relativity. We demonstrated that also a complex system like the brain can be tackled with symmetry principles and that the presence or absence of certain symmetries is crucial for the emergence of realistic patterns.

In Chapter 4 we studied how OD segregation influences the layout of OP maps. This study is the first to clearly demonstrate that inter-map coupling can lead to stabilization and even generation of pinwheels in OP maps [121]. With the presented methods we were able to identify the genuine ground states of each analyzed model and our analytical approach allowed for a thorough characterization of these states. The presented results question the widely held view that OD stripes are able to stabilize pinwheels. We demonstrated that the impact of OD stripes leads to pinwheel annihilation whereas a patchy OD layout can stabilize pinwheels, underlining the importance of the over-representation of one eye over the other. In Chapter 5 we integrated long-range connections among cortical neurons into the dynamics of OP maps. We showed that with the inclusion of such non-local interactions inter-map coupling leads to a patchy and spatially irregular OD layout resembling the layout observed in cat visual cortex. Moreover, we gave a potential explanation of inter-species differences observed in the OD layout. A detuning of OD and OP average wavelengths, as observed for instance in macaque monkeys, leads to a spatially irregular, although more stripe-like, OD layout which resembles that found in the macaque visual cortex.

Our study suggests a hierarchy of cortical maps where one map dominates the others. The most plausible models were identified as those in which the OP map dominates. We showed that there is a limit in which we can neglect a backreaction of the co-evolving map onto the OP map. We therefore studied in detail the uncoupled OP dynamics which has a remarkable property: A huge variety of stationary solutions which sets this dynamics apart from the dynamics of a real order parameter field such as that of the OD map. The inclusion of non-local interactions into the OP dynamics leads to a solution class with a vast number of multistable 
solutions which are energetically degenerate. Chapter 6 and Chapter 7 were devoted to the question of how this degeneracy is lifted. We characterized the numerous solutions, studied how the degeneracy can be lifted and how this impacts the stability borders and quantitative properties of each solution. So far, analytical results were obtained by amplitude equations in the leading order of a perturbative expansion near the bifurcation point. Here, the assumed permutation symmetry of the nonlinear interaction terms guarantees multistability and energetic degeneracy. How far the emergence of cortical structures takes place from criticality, however, is not clear from the experimental point of view. For this reason it is important to study pattern formation also substantially far from threshold. We therefore derived higher order corrections to amplitude equations of the uncoupled OP dynamics in Chapter 6. To our knowledge our study is the first one to calculate such higher order corrections in a highly multistable Swift-Hohenberg equation. We studied how these corrections alter the stationary patterns and in particular their pinwheel densities. We showed that these corrections energetically select solutions with a relatively low pinwheel density. In Chapter 7 we generalized the OP dynamics by breaking the permutation symmetry of the nonlinear terms. This lifts the degeneracy already at leading order. Moreover, with broken permutation symmetry the dominant class of stationary solutions, the ECP solutions, is accompanied by additional solution classes. From these new classes we identified stable solutions which have realistic pinwheel densities and are relatively insensitive to the model details. Thus breaking permutation symmetry can lead by energetically selecting ECP solutions with a high pinwheel density and the occurrence of additional solution classes to realistic patterns even far from threshold.

Routes for future investigations.

We demonstrated that inter-map coupling can lead to a crystallization of pinwheels into a regular lattice. We expect to overcome this spatial regularity for instance by imposing realistic boundary conditions [116] or by introducing inhomogeneities such as spatial variations in the typical wavelength [122]. To study such spatial variations in a perturbative approach, so called envelope equations have to be considered [120]. Another interesting possibility to study spatial inhomogeneities in the OP map results from the presented map interaction models. In this scenario the co-evolving field does not represent a feature map but describes an artificial border or disruption of OP, which can be induced also experimentally [123]. The presented analytical approach enables us to analyze map interactions even in high feature dimensions. We demonstrated that interactions among multiple maps can lead to frustration of different optimization constraints which can result in pinwheel crystallization. In dimension reduction models spatially regular patterns seem to be a common feature [100. In contrast, the introduced analytical approach enables us to identify biological constraints such as a bias towards certain stimuli that can lead to spatially irregular patterns even in high feature dimensions. In the presented models OP maps at intermediate stages of development are relatively insensitive to model details such as the distance to threshold. Maps at late stages, however, are very susceptible to model details. From the experimental point of view the intrinsic timescale in such models cannot precisely matched with the developmental stage 
of an animal. However, our results and predictions for pattern selection at late stages might guide future experiments. In particular, they might guide chronic long-term recordings in developing and adult animals which are experimentally very challenging. The description of OP map development with the use of a complex Swift-Hohenberg equation turned out to be very promising as it has numerous biologically plausible solutions. In particular, we demonstrated that breaking permutation symmetry is a first step to study new solution classes and introduced the CPP solutions which might in some models supersede the ECP solutions. The complex Swift-Hohenberg equation applies to many physical systems such as traveling waves in lasers [14] or the occurrence of defects in Rayleigh-Bénard convection [124]. The extended solution set to this equation we presented in this thesis is thus not only interesting for models of cortical map development.

The introduced models cover the basic design principles found in the visual cortex. Symmetries, the geometry of neuronal connectivity, and geometric inter-map relations framed a general model of visual cortical development. By varying a few phenomenological parameters we are able to study a wide range of phenomena found in visual cortical maps. In the end, there is the hope that these fundamental principles found in the visual system will to some degree trigger a better understanding of other parts of the brain. 


\section{Notation guide}

The following table is a quick-reference guide to the notation used in each chapter.

\begin{tabular}{|c|c|}
\hline Nomenclature & Meaning \\
\hline \multicolumn{2}{|l|}{ Chapter 2} \\
\hline OD & Ocular dominance \\
\hline OP & Orientation preference \\
\hline$o(\mathbf{x}, t)$ & Real order parameter field for OD map, see 2.2.1 \\
\hline$z(\mathbf{x}, t)$ & Complex order parameter field, see 2.2 .2 \\
\hline$\rho$ & $\begin{array}{c}\text { with } \vartheta=1 / 2 \arg z \text { the preferred orientation, }|z| \text { the selectivity } \\
\text { Pinwheel density (pinwheels per } \Lambda^{2} \text { ), see } 2.2 .2\end{array}$ \\
\hline \multicolumn{2}{|l|}{ Chapter 3} \\
\hline$\Lambda=2 \pi / k_{c}$ & Typical wavelength, see 3.1 \\
\hline$r_{z}, r_{o}$ & Bifurcation parameter for OP, OD map, see 3.1 \\
\hline$g$ & Measures local vs. non-local interactions, see 3.1 \\
\hline$\sigma$ & Width of non-local interactions, see 3.1 \\
\hline$U$ & Inter-map coupling energy, see 3.2 \\
\hline$\Gamma$ & Aspect ratio, $\Gamma=$ system size $/ \Lambda$, see 3.3 \\
\hline$\gamma$ & OD bias, see 4.1 \\
\hline$d$ & Nearest neighbor pinwheel distance, see 3.4 \\
\hline$c(t)$ & Pinwheel creation rate, see 3.4 \\
\hline$a(t)$ & Pinwheel annihilation rate, see 3.4 \\
\hline$s(t)$ & Fraction of surviving pinwheels, see 3.4 \\
\hline$p(t)$ & Fraction of final pinwheels present in the pattern, see 3.4 \\
\hline \multicolumn{2}{|l|}{ Chapter 4} \\
\hline$B=\mathcal{B} e^{\imath \psi}$ & Active modes and phases of OD map, see 4.2 .2 \\
\hline$A=\mathcal{A} e^{\imath \phi}$ & Active modes and phases of OP map, see 4.3 \\
\hline PWC & Pinwheel crystal \\
\hline \multicolumn{2}{|l|}{ Chapter 5} \\
\hline $\mathrm{ECP}$ & Essentially complex planform, see 5.3 \\
\hline$l_{j}$ & $= \pm 1$, Selects active mode with $\vec{k}_{j}$ or $-\vec{k}_{j}$, see 5.3 \\
\hline
\end{tabular}




\begin{tabular}{|c|c|}
$n$ & Number of active modes, also $n_{o}, n_{z}$, see 5.3 \\
LDP & Low pinwheel density planform, see [5.3 \\
HDP & High pinwheel density planform, see 5.3 \\
$\Delta$ & Classifies uniform solutions, see 4.4, 5.5, 7.2.2 \\
$\chi$ & Uniformity parameter of OD map, see 5.6.1 \\
$b$ & Bandedness of OD map, see 5.6.1 and Appendix \\
$\alpha$ & Intersection angle, see 5.8 \\
Chapter 6 & Planform anisotropy, see 6.6 \\
$\xi$ & Number of flipped mode clusters, see 6.6 \\
$\kappa$ & Circular phase progression planform, see 7.2 .2 \\
Chapter 7 & Binary phase planform, see 7.2 .3 \\
CPP &
\end{tabular}




\section{A. Appendix}

\section{A.1. Numerical integration method}

In this appendix we briefly sketch the numerical integration scheme which was implemented by D. Heide [116] and used in this thesis. As the Swift-Hohenberg equation is a stiff partial differential equation we used a fully implicit integrator [125. Such an integration scheme avoids numerical instabilities and enables the use of increasing stepsizes when approaching an attractor state. The equation

$$
\partial_{t} z(\mathbf{x}, t)=\hat{L} z(\mathbf{x}, t)-N[z(\mathbf{x}, t)], \quad \hat{L}=r-\left(k_{c}^{2}+\Delta\right)^{2},
$$

is discretized in time. Using a Crank-Nicolson scheme, this differential equation is approximated by the nonlinear difference equation

$$
\frac{z_{t+1}-z_{t}}{\Delta t}=\frac{\left(\hat{L} z_{t+1}+N\left[z_{t+1}\right]\right)+\left(\hat{L} z_{t}+N\left[z_{t}\right]\right)}{2} .
$$

This equation is solved iteratively for $z_{t+1}$ with the help of the Newton method which finds the root of the function

$$
G\left[z_{t+1}\right]=\left(-\hat{L}+\frac{2}{\Delta t}\right) z_{t+1}-N\left[z_{t+1}\right]-\left(\left(\hat{L}+\frac{2}{\Delta t}\right) z_{t}+N\left[z_{t}\right]\right) .
$$

The field $z(\mathbf{x})$ is discretized. For a grid with $N$ meshpoints in $x$-direction and $M$ meshpoints in $y$-direction this leads to a $M \times N$ dimensional state vector $\mathbf{u}$. Discretization is performed in Fourier space. The Newton iteration at step $k$ is then given by

$$
D G\left(u^{k}\right) \Delta \mathbf{u}^{k}=-G\left(\mathbf{u}^{k}\right), \quad \mathbf{u}^{k+1}=\mathbf{u}^{k}+\Delta \mathbf{u}^{k},
$$

with $D G$ the Jacobian of $G$. Instead of calculating the matrix $D G$ explicitly a matrix free method is used, where the action of the matrix is approximated using finite differences. To solve the linear system $A \mathbf{x}=\mathbf{b}$ with $A=D G\left(\mathbf{u}^{k}\right), \mathbf{b}=-G\left(\mathbf{u}^{k}\right)$ we used the Krylov subspace method [125]. The Krylov subspace of dimensionality $k$ is defined as

$$
\mathcal{K}_{k}\left(A, \mathbf{v}_{1}\right)=\operatorname{span}\left\{\mathbf{v}_{1}, A \mathbf{v}_{1}, A^{2} \mathbf{v}_{1}, \ldots, A^{k-1} \mathbf{v}_{1}\right\}
$$

In the Generalized Minimum Residual (GMRES) algorithm the Krylov subspace is generated by $\mathbf{v}_{1}=\mathbf{r}_{0} /\left|\mathbf{r}_{0}\right|$ with $\mathbf{r}_{0}=A \mathbf{x}_{0}-\mathbf{b}$, and $\mathbf{x}_{0}$ an initial guess, see [125. After $k$ iterations, the 
refined solution is given by

$$
\mathbf{x}_{k}=\mathbf{x}_{0}+V_{k} \mathbf{y}
$$

where the matrix $V_{k}=\left(\mathbf{v}_{1}, \ldots, \mathbf{v}_{k}\right)$ has the base vectors of the Krylov subspace as its columns. The vector $\mathbf{y}$ is chosen by minimizing the residuum

$$
\left\|b-A \mathbf{x}_{k}\right\|_{2}=\left\|r_{0}-A V_{k} \mathbf{y}\right\|_{2} \stackrel{!}{=} \min
$$

where $\|\cdot\|_{2}$ denotes the Euclidean norm. For this procedure an orthonormal basis of the Krylov subspace is generated with an Arnoldi process. With the use of the similarity transformation

$$
A V_{k}=V_{k+1} \tilde{H}_{k}
$$

where $\tilde{H}_{k}$ is an upper Hessenberg matrix, $\mathbf{v}_{1}=\mathbf{r}_{0} /\left|\mathbf{r}_{0}\right|$, and the orthogonality of $V_{k}$, the optimality condition Eq. (A-7) becomes

$$
\left\|\tilde{H}_{k} \mathbf{y}-|\mathbf{b}| \mathbf{e}_{1}\right\|_{2} \stackrel{!}{=} \min
$$

with $\mathbf{e}_{1}=(1,0, \ldots, 0)$ the first unit vector of dimension $k+1$. For a $\mathbf{y}$ that minimizes this norm the approximate solution is given by $\mathbf{x}_{k}=\mathbf{x}_{0}+V_{k} \mathbf{y}$. To improve the convergence of this iterative method preconditioning was used. A preconditioner $M$ is multiplied to $A \mathbf{x}=\mathbf{b}$ such that $M^{-1} A$ is close to unity. A preconditioner suitable for our model is the inverse of the linear operator in Fourier space with a small shift $0<\epsilon \ll 1$ in order to avoid singularities i.e.

$$
M=\left(\epsilon+\left(k^{2}-k_{c}^{2}\right)^{2}+\frac{2}{\Delta t}\right)^{-1} .
$$

The convergence of Newton's method is only guaranteed from a starting point close enough to a solution. In the integration scheme we use a line search method to ensure also a global convergence [126]. Newton's method Eq. (A-4) is thus modified as

$$
\mathbf{u}^{k+1}=\mathbf{u}^{k}+\lambda \Delta \mathbf{u}^{k}
$$

where the function

$$
f\left(\mathbf{u}^{k}\right)=\frac{1}{2} G\left(\mathbf{u}^{k}\right) G\left(\mathbf{u}^{k}\right),
$$

is iteratively minimized with respect to $\lambda$.

This integrator was implemented using the PetSc library [127]. As the dynamics converges towards an attractor an adaptive stepsize control is very efficient. The employed adaptive stepsize control was implemented as described in [128. The described integration scheme has been generalized for an arbitrary number of real or complex fields. The coupling terms are treated as additional nonlinearities in $N$. As a common intrinsic timescale we choose $T=t r_{z}$ with $r_{z}$ the bifurcation parameter of the OP map. Due to the spatial discretization not all points of the critical circle lie on the grid. Thus, the maximal growth rate on the discretized circle is not exactly equal to $r$, the theoretical growth rate. In particular, some 
modes may be suppressed or even become unstable. Due to this we expect deviations from analytical solutions we discussed in this thesis. To minimize such deviations the size of the critical circle was chosen such that this disbalance between the active modes was minimized. Periodic boundary conditions were applied to account for the translation invariance of the spatial pattern.

\section{A.2. Coupling coefficients I}

Here we list the explicit form of the coupling coefficients $l_{i j l k}^{(u)}$ of the coupled amplitude equa-

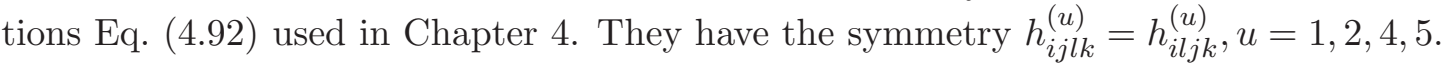

$$
\begin{array}{rc}
h_{i i i i}^{(1)}=40.5 \epsilon+90 \tau, & h_{i i i j}^{(1)}=(16.125 \epsilon+48 \tau) e^{\imath\left(\psi_{j}-\psi_{i}\right)}, \\
h_{i j j j}^{(1)}=(16.125 \epsilon+48 \tau) e^{\imath\left(\psi_{i}-\psi_{j}\right)}, & h_{i i j j}^{(1)}=40.5 \epsilon+180 \tau, \\
h_{i i j i}^{(1)}=(32.25 \epsilon+96 \tau) e^{\imath\left(\psi_{i}-\psi_{j}\right)}, & h_{i j j i}^{(1)}=(4.125 \epsilon+6 \tau) e^{\imath 2\left(\psi_{i}-\psi_{j}\right)}, \\
h_{i i j l}^{(1)}=-(16.5 \epsilon+96 \tau) e^{\imath\left(\psi_{l}-\psi_{j}\right)}, & h_{i j l i}^{(1)}=(7.5 \epsilon+24 \tau) e^{\imath\left(2 \psi_{i}-\psi_{j}-\psi_{l}\right)}, \\
h_{i j l l}^{(1)}=(16.5 \epsilon+96 \tau) e^{\imath\left(\psi_{i}-\psi_{j}\right)}, & h_{i j j l}^{(1)}=(3.75 \epsilon+12 \tau) e^{\imath\left(\psi_{l}+\psi_{i}-2 \psi_{j}\right)}
\end{array}
$$

In terms containing also the opposite wavevectors $A_{j-}$ triad resonances $\vec{k}_{1}-\vec{k}_{2}+\vec{k}_{3}=0$ appear. They lead to the phase relation $\psi_{1}+\psi_{2}+\psi_{3}=\pi$ for the modes $B_{j}$ and therefore the coupling coefficients depend only on two of the three phases.

$$
\begin{array}{rc}
h_{i i i i}^{(2)}=(19.25 \epsilon+34 \tau) e^{2 \imath \psi_{i}}, & h_{i j j j}^{(2)}=(15.75 \epsilon+60 \tau) e^{\imath\left(\psi_{i}+\psi_{j}\right)}, \\
h_{i i i j}^{(2)}=(3.5 \epsilon+4 \tau) e^{\imath\left(3 \psi_{i}-\psi_{j}\right)}, & h_{i i j i}^{(2)}=(31.5 \epsilon+120 \tau) e^{\imath\left(\psi_{i}+\psi_{j}\right)}, \\
h_{i j j i}^{(2)}=(9.875 \epsilon+34 \tau) e^{\imath \psi_{j}}, & h_{i i j j}^{(2)}=(19.75 \epsilon+68 \tau) e^{2 \imath \psi_{i}}, \\
h_{i i j l}^{(2)}=-(7.25 \epsilon+32 \tau) e^{\imath\left(3 \psi_{i}+2 \psi_{j}\right)}, & h_{i j l i}^{(2)}=(9 \epsilon+120 \tau) e^{\imath \psi_{i}}, \\
h_{i j l l}^{(2)}=(9 \epsilon+120 \tau) e^{\imath\left(\psi_{i}+\psi_{j}\right)}, & h_{i j j l}^{(2)}=-(3.625 \epsilon+16 \tau) e^{\imath\left(2 \psi_{i}+3 \psi_{j}\right)} \\
h_{i i i i}^{(3)}=(38.5 \epsilon+68 \tau) e^{2 \imath \psi_{i}}, & h_{i j j j}^{(3)}=h_{i i i j}^{(3)}=h_{i i j i}^{(3)}=(31.5 \epsilon+120 \tau) e^{\imath\left(\psi_{i}+\psi_{j}\right)} \\
h_{i j i i}^{(3)}=(7 \epsilon+8 \tau) e^{\imath\left(3 \psi_{i}-\psi_{j}\right)}, & h_{i i j j}^{(3)}=(19.75 \epsilon+68 \tau) e^{2 \imath \psi_{j}}, \\
h_{i j j i}^{(3)}=h_{i j i j}^{(3)}=(19.75 \epsilon+68 \tau) e^{2 \imath \psi_{i}}, & h_{i i j l}^{(3)}=-(9 \epsilon+120 \tau) e^{\imath \psi_{i}}, \\
h_{i j i l}^{(3)}=h_{i j l i}^{(3)}=-(7.25 \epsilon+32 \tau) e^{\imath\left(\psi_{i}-2 \psi_{j}\right)}, & h_{i j l l}^{(3)}=(7.25 \epsilon+32 \tau) e^{-\imath\left(\psi_{i}+3 \psi_{j}\right)}, \\
h_{i l j l}^{(3)}=(9 \epsilon+120 \tau) e^{\imath\left(\psi_{i}+\psi_{j}\right)}, & h_{i j j l}^{(3)}=-(9 \epsilon+120 \tau) e^{-\imath \psi_{j}}
\end{array}
$$




$$
\begin{aligned}
& h_{i i i i}^{(4)}=(2 \epsilon+\tau) e^{4 \imath \psi_{i}}, \quad h_{i j j j}^{(4)}=(3.125 \epsilon+16 \tau) e^{\imath\left(\psi_{i}+3 \psi_{j}\right)}, \\
& h_{i i i j}^{(4)}=(3.125 \epsilon+16 \tau) e^{\imath\left(3 \psi_{i}+\psi_{j}\right)}, \quad h_{i i j i}^{(4)}=2 h_{i i i j}^{(4)}, \\
& h_{i j j i}^{(4)}=(5.125 \epsilon+34 \tau) e^{2 \imath\left(\psi_{i}+\psi_{j}\right)}, \quad h_{i i j j}^{(4)}=2 h_{i j j i}^{(4)} \text {, } \\
& h_{i i j l}^{(4)}=h_{i j l i}^{(4)}=(9 \epsilon-120 \tau) e^{\imath \psi_{i}}, \quad h_{i j l l}^{(4)}=(-9 \epsilon+120 \tau) e^{-\imath\left(\psi_{i}+\psi_{j}\right)}, \\
& h_{i j j l}^{(4)}=(4.5 \epsilon-60 \tau) e^{\imath \psi_{j}} \\
& h_{i i i i}^{(5)}=(19.25 \epsilon+34 \tau) e^{-2 \imath \psi_{i}}, \quad h_{i j j j}^{(5)}=(3.5 \epsilon+4 \tau) e^{\imath\left(\psi_{i}-3 \psi_{j}\right)}, \\
& h_{i i i j}^{(5)}=(15.75 \epsilon+60 \tau) e^{-\imath\left(\psi_{i}+\psi_{j}\right)}, \quad h_{i i j i}^{(5)}=2 h_{i i i j}^{(5)}, \\
& h_{i j j i}^{(5)}=(9.875 \epsilon+34 \tau) e^{-2 \imath \psi_{j}}, \quad h_{i i j j}^{(5)}=2 h_{i j j i}^{(5)}, \\
& h_{i j l l}^{(5)}=(7.25 \epsilon+32 \tau) e^{\imath\left(3 \psi_{i}+\psi_{j}\right)}, \quad h_{i j j l}^{(5)}=-(3.625 \epsilon+16 \tau) e^{\imath\left(2 \psi_{i}-\psi_{j}\right)} \text {, } \\
& h_{i i j l}^{(5)}=h_{i j l i}^{(5)}=-(9 \epsilon+120 \tau) e^{\imath \psi_{i}} \\
& h_{i i i i}^{(6)}=(2 \epsilon+180 \tau) e^{\imath \psi_{i}}, \quad h_{i i j i}^{(6)}=(32.25 \epsilon+96 \tau) e^{\imath\left(-\psi_{i}+\psi_{j}\right)}, \\
& h_{i j j j}^{(6)}=h_{i i i j}^{(6)}=h_{i j i i}^{(6)}=(32.25 \epsilon+96 \tau) e^{\imath\left(\psi_{i}-\psi_{j}\right)} \text {, } \\
& h_{i j j i}^{(6)}=h_{i i j j}^{(6)}=(40.5 \epsilon+180 \tau), \quad h_{i j i j}^{(6)}=(8.25 \epsilon+12 \tau) e^{2 \imath\left(\psi_{i}-\psi_{j}\right)}, \\
& h_{i j i l}^{(6)}=-(7.5 \epsilon+24 \tau) e^{\imath 3 \psi_{i}}, \quad h_{i l j l}^{(6)}=(7.5 \epsilon+24 \tau) e^{3 \imath\left(\psi_{i}+\psi_{j}\right)}, \\
& h_{i j l l}^{(6)}=(16.5 \epsilon+96 \tau) e^{\imath\left(\psi_{i}-\psi_{j}\right)}, \quad h_{i j j l}^{(6)}=-(16.5 \epsilon+96 \tau) e^{-\imath\left(2 \psi_{i}+\psi_{j}\right)} \\
& h_{i i j l}^{(6)}=h_{i j l i}^{(6)}=-(16.5 \epsilon+96 \tau) e^{\imath\left(\psi_{i}+2 \psi_{j}\right)}
\end{aligned}
$$

\section{A.3. Coupling coefficients II}

Here we list the coupling coefficients for the amplitude equations Eq. (5.20). We define $p_{i, j}=(i-j) \frac{2 \pi}{n}$. The couplings terms defined in Eq. (5.17) are given by

$$
\begin{aligned}
h_{i j l k}= & \epsilon\left(16+8 \cos p_{i, j}+12 \cos p_{i, k}\right. \\
& +12 \cos p_{j, k}+12 \cos p_{i, l}+8 \cos p_{k, l}+12 \cos p_{j, l} \\
& \left.+12 \cos p_{i+j, l+k}+2 \cos p_{i+k, j+l}+2 \cos p_{i+l, j+k}\right)+48 \tau \\
h_{i j l l}= & \epsilon\left(6+3 \cos p_{i, j}+6 \cos p_{i, l}\right. \\
& \left.+6 \cos p_{j, l}+3 \cos p_{i+j, 2 l}\right)+12 \tau \\
h_{i i j l}= & 2 h_{l j i i} \\
h_{i i l l}= & \epsilon\left(\frac{9}{2}+6 \cos p_{i, l}+\frac{3}{2} \cos p_{2 i, 2 l}\right)+6 \tau
\end{aligned}
$$


The additional coupling terms that appear in Eq. (5.20) are given by

$$
\begin{aligned}
& l_{i j j j}=15 \epsilon+9 \epsilon \cos p_{i, j}+12 \tau, \quad l_{i j i i}=l_{i j j j} \\
& l_{i i j i}=2 l_{i j j j}, \quad l_{i i i l}=l_{i i l i} \\
& l_{\text {iill }}=\epsilon\left(\frac{27}{4}+5 \cos p_{i, l}+\frac{1}{4} \cos p_{2 i, 2 l}\right)+6 \tau \\
& l_{i j l l}=\epsilon\left(10+\frac{7}{2} \cos p_{i, j}+5 \cos p_{i, l}+5 \cos p_{j, l}+\frac{1}{2} \cos p_{i+j, 2 l}\right)+12 \tau \\
& l_{i j l i}=4 l_{i l j j}, \quad l_{i j j l}=l_{i j l j} \\
& l_{i j l i}=l_{j i i l}, \quad l_{i i j l}=\frac{1}{2} l_{i j l i} \\
& l_{i j l k}=\epsilon\left(30+10 \cos p_{i, j}+10 \cos p_{i, k}+10 \cos p_{j, k}\right. \\
& +10 \cos p_{i, l}+10 \cos p_{j, l}+10 \cos p_{k, l} \\
& \left.+2 \cos p_{i+j, l+k}+2 \cos p_{i+k, j+l}+2 \cos p_{i+l, j+k}\right)+48 \tau \\
& \lambda_{i i j j}=\epsilon\left(\frac{45}{2}+24 \cos p_{i, j}+\frac{3}{2} \cos p_{2 i, 2 j}\right)+24 \tau, \quad \lambda_{i j i j}=\frac{1}{2} \lambda_{i i j j} \\
& \lambda_{i j l l}=\epsilon\left(15+\frac{15}{2} \cos p_{i, j}+15 \cos p_{i, l}+9 \cos p_{j, l}+\frac{3}{2} \cos p_{i+j, 2 l}\right)+24 \tau \\
& \lambda_{i l j l}=\lambda_{i j l l}, \lambda_{i j i l}=\lambda_{l j i i}, \lambda_{i i j l}=2 \lambda_{l j i i} \\
& \lambda_{i j l k}=\epsilon\left(20+10 \cos p_{i, j}+10 \cos p_{j, k}\right. \\
& +8 \cos p_{j, l}+20 \cos p_{i, k}+10 \cos p_{i, l} \\
& +10 \cos p_{k, l}+3 \cos p_{i+j, l+k} \\
& \left.+3 \cos p_{i+l, j+k}+2 \cos p_{i+k, j+l}\right)+48 \tau \\
& \rho_{i j j i}=\epsilon\left(\frac{45}{2}+24 \cos p_{i, j}+\frac{3}{2} \cos p_{2 i, 2 j}\right)+24 \tau \\
& \rho_{i j l l}=\epsilon\left(15+\frac{15}{2} \epsilon \cos p_{i, j}+15 \cos p_{j, l}+9 \cos p_{i, l}+\frac{3}{2} \cos p_{i+j, 2 l}\right)+24 \tau \\
& \rho_{l j i l}=\rho_{i j l l} \\
& \rho_{i j l k}=\epsilon\left(20+10 \cos p_{i, j}+8 \cos p_{i, l}+10 p_{i, k}\right. \\
& +10 \cos p_{j, l}+20 \cos p_{j, k} \\
& +10 \cos p_{k, l}+3 \cos p_{i+j, l+k} \\
& \left.+2 \cos p_{i+l, j+k}+3 \cos p_{i+k, j+l}\right)+48 \tau
\end{aligned}
$$

\section{A.4. Calculation of the bandedness}

In this appendix we briefly sketch the derivation of the bandedness used in chapter 5. The calculation of the bandedness is based on a wavelet transformation [79]. The transformation 
of the pattern $I(\mathbf{y})$ is given by

$$
\hat{I}(\mathbf{x}, \theta, l)=\int d^{2} y I(\mathbf{y}) \Psi_{\mathbf{x}, \theta, l}(\mathbf{y}),
$$

where the wavelet at position $\mathbf{x}$, orientation $\theta$, and scale $l$ is given by

$$
\Psi_{\mathbf{x}, \theta, l}(\mathbf{y})=l^{-1} \Psi\left(\Omega^{-1}(\theta) \frac{y-x}{l}\right),
$$

with the anisotropic Gaussian

$$
\Psi(\mathbf{x})=\exp \left(-\frac{1}{2} \mathbf{x}^{T}\left(\begin{array}{cc}
1 & 0 \\
0 & \sigma_{y}^{2}
\end{array}\right) \mathbf{x}\right) e^{\imath k_{\Psi} \mathbf{x}}
$$

and the rotation matrix $\Omega(\theta)=\left(\begin{array}{cc}\cos \theta & -\sin \theta \\ \sin \theta & \cos \theta\end{array}\right)$. With the use of the wavelet coefficients $\hat{I}$ we first calculate the average wavelength $\Lambda$ of the pattern. Here we use an isotropic wavelet $\left(\sigma_{y}=1\right)$ with about seven lobes, $k_{\Psi}=(7,0)$. The orientation averaged modulus is given by

$$
\bar{I}=\int_{0}^{\pi} \frac{d \theta}{\pi}|\hat{I}(\mathbf{x}, \theta, l)|
$$

The scale $\bar{l}$ that maximizes $\bar{I}$ is determined by $\bar{l}=\arg \max (\bar{I})$. The column spacing finally is given by $\Lambda(\mathbf{x})=\bar{l} \Lambda_{\Psi}$ with $\Lambda_{\Psi}=2 \pi /\left|k_{\Psi}\right|$. The mean column spacing is given by $\Lambda=\langle\Lambda(\mathbf{x})\rangle_{x}$. Next we calculate the bandedness of the pattern. For the degree of anisotropy we take $\sigma_{y}=1.5$ and a wavelet with about two lobes, $k_{\Psi}=(2,0)$. The orientation dependence of the wavelet coefficient is given by

$$
s^{\prime}(\mathbf{x})=\int_{0}^{\pi} d \theta|\hat{I}(\mathbf{x}, \theta)|^{2} e^{2 \imath \theta} / \int_{0}^{\pi} d \theta|\tilde{o}(\mathbf{x}, \theta)|^{2} .
$$

The local average of this quantity is given by

$$
s(\mathbf{x})=\int d^{2} x^{\prime} s^{\prime}\left(\mathbf{x}^{\prime}\right) K\left(\mathbf{x}^{\prime}-\mathbf{x}\right) / \int d^{2} x^{\prime} K\left(\mathbf{x}^{\prime}-\mathbf{x}\right)
$$

with the kernel $K(\mathbf{x})=\frac{1}{2 \pi \sigma^{2}} e^{-\mathbf{x}^{2} / 2 \sigma^{2}}$. The width is chosen as $\sigma=1.3\langle\Lambda(\mathbf{x})\rangle_{x}$. The local bandedness is given by the absolute value $|s(\mathbf{x})|$ and the mean bandedness is given by the spatial average $b=\langle|s(\mathbf{x})|\rangle_{\mathbf{x}}$.

\section{A.5. Fifth order coupling coefficients}

Here we list the coupling coefficients of the fifth order amplitude equations Eq. (6.16).

$$
\begin{aligned}
g_{i i i} & =0 \\
g_{i i i^{-}} & =e^{-\imath \vec{k}_{i} \vec{x}}\left(N_{3}\left[\left(\hat{L}^{0}\right)^{-1} N_{3}\left[e^{\imath \vec{k}_{i} \vec{x}}, e^{\imath \vec{k}_{i} \vec{x}}, e^{\imath \vec{k}_{i} \vec{x}}\right], e^{-\imath \vec{k}_{i} \vec{x}}, e^{-\imath \vec{k}_{i} \vec{x}}\right]+\right.
\end{aligned}
$$




$$
\begin{aligned}
& \left.N_{3}\left[e^{-\imath \vec{k}_{i} \vec{x}},\left(\hat{L}^{0}\right)^{-1} N_{3}\left[e^{\imath \vec{k}_{i} \vec{x}}, e^{\imath \vec{k}_{i} \vec{x}}, e^{\imath \vec{k}_{i} \vec{x}}\right], e^{-\imath \vec{k}_{i} \vec{x}}\right]\right) \\
& g_{i i j}=e^{-\imath \vec{k}_{i} \vec{x}}\left(N_{3}\left[\left(\hat{L}^{0}\right)^{-1} N_{3}\left[e^{\imath \vec{k}_{i} \vec{x}}, e^{\imath \vec{k}_{i} \vec{x}}, e^{-\imath \vec{k}_{j} \vec{x}}\right], e^{\imath \vec{k}_{j} \vec{x}}, e^{-\imath \vec{k}_{i} \vec{x}}\right]+\right. \\
& \left.N_{3}\left[e^{\imath \vec{k}_{j} \vec{x}},\left(\hat{L}^{0}\right)^{-1} N_{3}\left[e^{\imath \vec{k}_{i} \vec{x}}, e^{\imath \vec{k}_{i} \vec{x}}, e^{-\imath \vec{k}_{j} \vec{x}}\right], e^{-\imath \vec{k}_{i} \vec{x}}\right]\right) \\
& g_{i i^{-} i^{-}}=e^{-\imath \vec{k}_{i} \vec{x}} N_{3}\left[e^{-\imath \vec{k}_{i} \vec{x}}, e^{-\imath \vec{k}_{i} \vec{x}},\left(\hat{L}^{0}\right)^{-1} N_{3}\left[e^{\imath \vec{k}_{i} \vec{x}}, e^{\imath \vec{k}_{i} \vec{x}}, e^{\imath \vec{k}_{i} \vec{x}}\right]\right] \\
& g_{i j j}=e^{-\imath \vec{k}_{i} \vec{x}} N_{3}\left[e^{\imath \vec{k}_{j} \vec{x}}, e^{\imath \vec{k}_{j} \vec{x}},\left(\hat{L}^{0}\right)^{-1} N_{3}\left[e^{-\imath \vec{k}_{j} \vec{x}}, e^{-\imath \vec{k}_{j} \vec{x}}, e^{\imath \vec{k}_{i} \vec{x}}\right]\right] \\
& g_{i j j^{-}}=e^{-\imath \vec{k}_{i} \vec{x}}\left(N_{3}\left[\left(\hat{L}^{0}\right)^{-1} N_{3}\left[e^{\imath \vec{k}_{i} \vec{x}}, e^{\imath \vec{k}_{j} \vec{x}}, e^{\imath \vec{k}_{j} \vec{x}}\right], e^{-\imath \vec{k}_{j} \vec{x}}, e^{-\imath \vec{k}_{j} \vec{x}}\right]+\right. \\
& N_{3}\left[\left(\hat{L}^{0}\right)^{-1} N_{3}\left[e^{\imath \vec{k}_{j} \vec{x}}, e^{\imath \vec{k}_{i} \vec{x}}, e^{\imath \vec{k}_{j} \vec{x}}\right], e^{-\imath \vec{k}_{j} \vec{x}}, e^{-\imath \vec{k}_{j} \vec{x}}\right]+ \\
& N_{3}\left[\left(\hat{L}^{0}\right)^{-1} N_{3}\left[e^{i \vec{k}_{i} \vec{x}}, e^{-\imath \vec{k}_{j} \vec{x}}, e^{-\imath \vec{k}_{j} \vec{x}}\right], e^{i \vec{k}_{j} \vec{x}}, e^{\imath \vec{k}_{j} \vec{x}}\right]+ \\
& N_{3}\left[\left(\hat{L}^{0}\right)^{-1} N_{3}\left[e^{-\imath \vec{k}_{j} \vec{x}}, e^{\imath \vec{k}_{i} \vec{x}}, e^{-\imath \vec{k}_{j} \vec{x}}\right], e^{\imath \vec{k}_{j} \vec{x}}, e^{\imath \vec{k}_{j} \vec{x}}\right]+ \\
& N_{3}\left[e^{-\imath \vec{k}_{j} \vec{x}},\left(\hat{L}^{0}\right)^{-1} N_{3}\left[e^{\imath \vec{k}_{i} \vec{x}}, e^{\imath \vec{k}_{j} \vec{x}}, e^{\imath \vec{k}_{j} \vec{x}}\right], e^{-\imath \vec{k}_{j} \vec{x}}\right]+ \\
& N_{3}\left[e^{-\imath \vec{k}_{j} \vec{x}},\left(\hat{L}^{0}\right)^{-1} N_{3}\left[e^{\imath \vec{k}_{j} \vec{x}}, e^{\imath \vec{k}_{i} \vec{x}}, e^{\imath \vec{k}_{j} \vec{x}}\right], e^{-\imath \vec{k}_{j} \vec{x}}\right]+ \\
& N_{3}\left[e^{\imath \vec{k}_{j} \vec{x}},\left(\hat{L}^{0}\right)^{-1} N_{3}\left[e^{\imath \vec{k}_{i} \vec{x}}, e^{-\imath \vec{k}_{j} \vec{x}}, e^{-\imath \vec{k}_{j} \vec{x}}\right], e^{\imath \vec{k}_{j} \vec{x}}\right]+ \\
& \left.N_{3}\left[e^{\imath \vec{k}_{j} \vec{x}},\left(\hat{L}^{0}\right)^{-1} N_{3}\left[e^{-\imath \vec{k}_{j} \vec{x}}, e^{\imath \vec{k}_{i} \vec{x}}, e^{-\imath \vec{k}_{j} \vec{x}}\right], e^{\imath \vec{k}_{j} \vec{x}}\right]\right) \\
& g_{i i^{-} j}=e^{-\imath \vec{k}_{i} \vec{x}}\left(N_{3}\left[e^{-\imath \vec{k}_{i} \vec{x}}, e^{\imath \vec{k}_{j} \vec{x}},\left(\hat{L}^{0}\right)^{-1} N_{3}\left[e^{-\imath \vec{k}_{j} \vec{x}}, e^{\imath \vec{k}_{i} \vec{x}}, e^{\imath \vec{k}_{i} \vec{x}}\right]\right]+\right. \\
& N_{3}\left[e^{\imath \vec{k}_{j} \vec{x}}, e^{-\imath \vec{k}_{i} \vec{x}},\left(\hat{L}^{0}\right)^{-1} N_{3}\left[e^{-\imath \vec{k}_{j} \vec{x}}, e^{\imath \vec{k}_{i} \vec{x}}, e^{\imath \vec{k}_{i} \vec{x}}\right]\right]+ \\
& N_{3}\left[e^{-\imath \vec{k}_{i} \vec{x}}, e^{\imath \vec{k}_{j} \vec{x}},\left(\hat{L}^{0}\right)^{-1} N_{3}\left[e^{\imath \vec{k}_{i} \vec{x}}, e^{-\imath \vec{k}_{j} \vec{x}}, e^{\imath \vec{k}_{i} \vec{x}}\right]\right]+ \\
& N_{3}\left[e^{\imath \vec{k}_{j} \vec{x}}, e^{-\imath \vec{k}_{i} \vec{x}},\left(\hat{L}^{0}\right)^{-1} N_{3}\left[e^{\imath \vec{k}_{i} \vec{x}}, e^{-\imath \vec{k}_{j} \vec{x}}, e^{\imath \vec{k}_{i} \vec{x}}\right]\right]+ \\
& N_{3}\left[e^{-\imath \vec{k}_{i} \vec{x}},\left(\hat{L}^{0}\right)^{-1} N_{3}\left[e^{\imath \vec{k}_{i} \vec{x}}, e^{\imath \vec{k}_{j} \vec{x}}, e^{\imath \vec{k}_{i} \vec{x}}\right], e^{-\imath \vec{k}_{j} \vec{x}}\right]+ \\
& N_{3}\left[e^{-\imath \vec{k}_{i} \vec{x}},\left(\hat{L}^{0}\right)^{-1} N_{3}\left[e^{\imath \vec{k}_{j} \vec{x}}, e^{\imath \vec{k}_{i} \vec{x}}, e^{\imath \vec{k}_{i} \vec{x}}\right], e^{-\imath \vec{k}_{j} \vec{x}}\right]+ \\
& N_{3}\left[\left(\hat{L}^{0}\right)^{-1} N_{3}\left[e^{\imath \vec{k}_{i} \vec{x}}, e^{\imath \vec{k}_{j} \vec{x}}, e^{\imath \vec{k}_{i} \vec{x}}\right], e^{-\imath \vec{k}_{i} \vec{x}}, e^{-\imath \vec{k}_{j} \vec{x}}\right]+ \\
& \left.N_{3}\left[\left(\hat{L}^{0}\right)^{-1} N_{3}\left[e^{\imath \vec{k}_{j} \vec{x}}, e^{\imath \vec{k}_{i} \vec{x}}, e^{\imath \vec{k}_{i} \vec{x}}\right], e^{-\imath \vec{k}_{i} \vec{x}}, e^{-\imath \vec{k}_{j} \vec{x}}\right]\right) \\
& g_{i j k}=e^{-\imath \vec{k}_{i} \vec{x}}\left(N_{3}\left[\left(\hat{L}^{0}\right)^{-1} N_{3}\left[e^{\imath \vec{k}_{i} \vec{x}}, e^{\imath \vec{k}_{j} \vec{x}}, e^{-\imath \vec{k}_{k} \vec{x}}\right], e^{\imath \vec{k}_{k} \vec{x}}, e^{-\imath \vec{k}_{j} \vec{x}}\right]+\right. \\
& N_{3}\left[\left(\hat{L}^{0}\right)^{-1} N_{3}\left[e^{\imath \vec{k}_{j} \vec{x}}, e^{\imath \vec{k}_{i} \vec{x}}, e^{-\imath \vec{k}_{k} \vec{x}}\right], e^{\imath \vec{k}_{k} \vec{x}}, e^{-\imath \vec{k}_{j} \vec{x}}\right]+ \\
& N_{3}\left[\left(\hat{L}^{0}\right)^{-1} N_{3}\left[e^{\imath \vec{k}_{i} \vec{x}}, e^{\imath \vec{k}_{k} \vec{x}}, e^{-\imath \vec{k}_{j} \vec{x}}\right], e^{i \vec{k}_{j} \vec{x}}, e^{-\imath \vec{k}_{k} \vec{x}}\right]+ \\
& N_{3}\left[\left(\hat{L}^{0}\right)^{-1} N_{3}\left[e^{\imath \vec{k}_{k} \vec{x}}, e^{\imath \vec{k}_{i} \vec{x}}, e^{-\imath \vec{k}_{j} \vec{x}}\right], e^{i \vec{k}_{j} \vec{x}}, e^{-\imath \vec{k}_{k} \vec{x}}\right]+
\end{aligned}
$$




$$
\begin{aligned}
& N_{3}\left[e^{\imath \vec{k}_{k} \vec{x}},\left(\hat{L}^{0}\right)^{-1} N_{3}\left[e^{\imath \vec{k}_{i} \vec{x}}, e^{\imath \vec{k}_{j} \vec{x}}, e^{-\imath \vec{k}_{k} \vec{x}}\right], e^{-\imath \vec{k}_{j} \vec{x}}\right]+ \\
& N_{3}\left[e^{\imath \vec{k}_{j} \vec{x}},\left(\hat{L}^{0}\right)^{-1} N_{3}\left[e^{\imath \vec{k}_{i} \vec{x}}, e^{\imath \vec{k}_{k} \vec{x}}, e^{-\imath \vec{k}_{j} \vec{x}}\right], e^{-\imath \vec{k}_{k} \vec{x}}\right]+ \\
& N_{3}\left[e^{\imath \vec{k}_{k} \vec{x}},\left(\hat{L}^{0}\right)^{-1} N_{3}\left[e^{\imath \vec{k}_{j} \vec{x}}, e^{\imath \vec{k}_{i} \vec{x}}, e^{-\imath \vec{k}_{k} \vec{x}}\right], e^{-\imath \vec{k}_{j} \vec{x}}\right]+ \\
& N_{3}\left[e^{\imath \vec{k}_{j} \vec{x}},\left(\hat{L}^{0}\right)^{-1} N_{3}\left[e^{\imath \vec{k}_{k} \vec{x}}, e^{\imath \vec{k}_{i} \vec{x}}, e^{-\imath \vec{k}_{j} \vec{x}}\right], e^{-\imath \vec{k}_{k} \vec{x}}\right]+ \\
& N_{3}\left[e^{\imath \vec{k}_{j} \vec{x}}, e^{\imath \vec{k}_{k} \vec{x}},\left(\hat{L}^{0}\right)^{-1} N_{3}\left[e^{-\imath \vec{k}_{j} \vec{x}}, e^{-\imath \vec{k}_{k} \vec{x}}, e^{\imath \vec{k}_{i} \vec{x}}\right]\right]+ \\
& N_{3}\left[e^{\imath \vec{k}_{k} \vec{x}}, e^{\imath \vec{k}_{j} \vec{x}},\left(\hat{L}^{0}\right)^{-1} N_{3}\left[e^{-\imath \vec{k}_{k} \vec{x}}, e^{-\imath \vec{k}_{j} \vec{x}}, e^{\imath \vec{k}_{i} \vec{x}}\right]\right]+ \\
& N_{3}\left[e^{\imath \vec{k}_{j} \vec{x}}, e^{\imath \vec{k}_{k} \vec{x}},\left(\hat{L}^{0}\right)^{-1} N_{3}\left[e^{-\imath \vec{k}_{k} \vec{x}}, e^{-\imath \vec{k}_{j} \vec{x}}, e^{\imath \vec{k}_{i} \vec{x}}\right]\right]+ \\
& \left.N_{3}\left[e^{\imath \vec{k}_{k} \vec{x}}, e^{\imath \vec{k}_{j} \vec{x}},\left(\hat{L}^{0}\right)^{-1} N_{3}\left[e^{-\imath \vec{k}_{j} \vec{x}}, e^{-\imath \vec{k}_{k} \vec{x}}, e^{\imath \vec{k}_{i} \vec{x}}\right]\right]\right) \\
& g_{i i j^{-}}=g_{i i j}\left(\vec{k}_{j} \rightarrow-\vec{k}_{j}\right), g_{i j^{-} j^{-}}=g_{i j j}\left(\vec{k}_{j} \rightarrow-\vec{k}_{j}\right), g_{i i^{-} j^{-}}=g_{i i-j}\left(\vec{k}_{j} \rightarrow-\vec{k}_{j}\right) \\
& f_{i i i}=f_{i i j}=f_{i i j^{-}}=0 \\
& f_{i j j}=e^{-\imath \vec{k}_{i} \vec{x}}\left(N_{3}\left[\left(\hat{L}^{0}\right)^{-1} N_{3}\left[e^{\imath \vec{k}_{j} \vec{x}}, e^{\imath \vec{k}_{j} \vec{x}}, e^{\imath \vec{k}_{i} \vec{x}}\right], e^{-\imath \vec{k}_{j} \vec{x}}, e^{-\imath \vec{k}_{j} \vec{x}}\right]+\right. \\
& N_{3}\left[e^{-\imath \vec{k}_{j} \vec{x}},\left(\hat{L}^{0}\right)^{-1} N_{3}\left[e^{\imath \vec{k}_{j} \vec{x}}, e^{\imath \vec{k}_{j} \vec{x}}, e^{\imath \vec{k}_{i} \vec{x}}\right], e^{-\imath \vec{k}_{j} \vec{x}}\right]+ \\
& N_{3}\left[e^{\imath \vec{k}_{j} \vec{x}}, e^{\imath \vec{k}_{j} \vec{x}},\left(\hat{L}^{0}\right)^{-1} N_{3}\left[e^{\imath \vec{k}_{i} \vec{x}}, e^{-\imath \vec{k}_{j} \vec{x}}, e^{-\imath \vec{k}_{j} \vec{x}}\right]\right]+ \\
& \left.N_{3}\left[e^{i \vec{k}_{j} \vec{x}}, e^{i \vec{k}_{j} \vec{x}},\left(\hat{L}^{0}\right)^{-1} N_{3}\left[e^{-\imath \vec{k}_{j} \vec{x}}, e^{\imath \vec{k}_{i} \vec{x}}, e^{-\imath \vec{k}_{j} \vec{x}}\right]\right]\right) \\
& f_{i j i^{-}}=e^{-\imath \vec{k}_{i} \vec{x}}\left(N_{3}\left[e^{-\imath \vec{k}_{i} \vec{x}}, e^{\imath \vec{k}_{j} \vec{x}},\left(\hat{L}^{0}\right)^{-1} N_{3}\left[e^{\imath \vec{k}_{i} \vec{x}}, e^{\imath \vec{k}_{i} \vec{x}}, e^{-\imath \vec{k}_{j} \vec{x}}\right]\right]\right. \\
& N_{3}\left[e^{\imath \vec{k}_{j} \vec{x}}, e^{-\imath \vec{k}_{i} \vec{x}},\left(\hat{L}^{0}\right)^{-1} N_{3}\left[e^{\imath \vec{k}_{i} \vec{x}}, e^{\imath \vec{k}_{i} \vec{x}}, e^{-\imath \vec{k}_{j} \vec{x}}\right]\right]+ \\
& N_{3}\left[e^{-\imath \vec{k}_{i} \vec{x}}, e^{-\imath \vec{k}_{j} \vec{x}},\left(\hat{L}^{0}\right)^{-1} N_{3}\left[e^{\imath \vec{k}_{i} \vec{x}}, e^{\imath \vec{k}_{i} \vec{x}}, e^{\imath \vec{k}_{j} \vec{x}}\right]\right]+ \\
& \left.N_{3}\left[e^{-\imath \vec{k}_{j} \vec{x}}, e^{-\imath \vec{k}_{i} \vec{x}},\left(\hat{L}^{0}\right)^{-1} N_{3}\left[e^{\imath \vec{k}_{i} \vec{x}}, e^{\imath \vec{k}_{i} \vec{x}}, e^{\imath \vec{k}_{j} \vec{x}}\right]\right]\right) \\
& f_{i j i}=e^{-\imath \vec{k}_{i} \vec{x}}\left(N_{3}\left[\left(\hat{L}^{0}\right)^{-1} N_{3}\left[e^{\imath \vec{k}_{j} \vec{x}}, e^{\imath \vec{k}_{i} \vec{x}}, e^{\imath \vec{k}_{i} \vec{x}}\right], e^{-\imath \vec{k}_{j} \vec{x}}, e^{-\imath \vec{k}_{i} \vec{x}}\right]+\right. \\
& N_{3}\left[\left(\hat{L}^{0}\right)^{-1} N_{3}\left[e^{\imath \vec{k}_{i} \vec{x}}, e^{\imath \vec{k}_{j} \vec{x}}, e^{\imath \vec{k}_{i} \vec{x}}\right], e^{-\imath \vec{k}_{j} \vec{x}}, e^{-\imath \vec{k}_{i} \vec{x}}\right]+ \\
& N_{3}\left[\left(\hat{L}^{0}\right)^{-1} N_{3}\left[e^{-\imath \vec{k}_{j} \vec{x}}, e^{\imath \vec{k}_{i} \vec{x}}, e^{\imath \vec{k}_{i} \vec{x}}\right], e^{\imath \vec{k}_{j} \vec{x}}, e^{-\imath \vec{k}_{i} \vec{x}}\right]+ \\
& N_{3}\left[\left(\hat{L}^{0}\right)^{-1} N_{3}\left[e^{\imath \vec{k}_{i} \vec{x}}, e^{-\imath \vec{k}_{j} \vec{x}}, e^{\imath \vec{k}_{i} \vec{x}}\right], e^{\imath \vec{k}_{j} \vec{x}}, e^{-\imath \vec{k}_{i} \vec{x}}\right]+ \\
& N_{3}\left[e^{-\imath \vec{k}_{j} \vec{x}},\left(\hat{L}^{0}\right)^{-1} N_{3}\left[e^{\imath \vec{k}_{j} \vec{x}}, e^{\imath \vec{k}_{i} \vec{x}}, e^{\imath \vec{k}_{i} \vec{x}}\right], e^{-\imath \vec{k}_{i} \vec{x}}\right]+ \\
& N_{3}\left[e^{-\imath \vec{k}_{j} \vec{x}},\left(\hat{L}^{0}\right)^{-1} N_{3}\left[e^{\imath \vec{k}_{i} \vec{x}}, e^{\imath \vec{k}_{j} \vec{x}}, e^{\imath \vec{k}_{i} \vec{x}}\right], e^{-\imath \vec{k}_{i} \vec{x}}\right]+ \\
& N_{3}\left[e^{\imath \vec{k}_{j} \vec{x}},\left(\hat{L}^{0}\right)^{-1} N_{3}\left[e^{-\imath \vec{k}_{j} \vec{x}}, e^{\imath \vec{k}_{i} \vec{x}}, e^{\imath \vec{k}_{i} \vec{x}}\right], e^{-\imath \vec{k}_{i} \vec{x}}\right]+
\end{aligned}
$$




$$
\begin{aligned}
& \left.N_{3}\left[e^{\imath \vec{k}_{j} \vec{x}},\left(\hat{L}^{0}\right)^{-1} N_{3}\left[e^{\imath \vec{k}_{i} \vec{x}}, e^{-\imath \vec{k}_{j} \vec{x}}, e^{\imath \vec{k}_{i} \vec{x}}\right], e^{-i \vec{k}_{i} \vec{x}}\right]\right) \\
& f_{i j k}=e^{-\imath \vec{k}_{i} \vec{x}}\left(N_{3}\left[e^{\imath \vec{k}_{k} \vec{x}}, e^{\imath \vec{k}_{j} \vec{x}},\left(\hat{L}^{0}\right)^{-1} N_{3}\left[e^{-\imath \vec{k}_{k} \vec{x}}, e^{\imath \vec{k}_{i} \vec{x}}, e^{-\imath \vec{k}_{j} \vec{x}}\right]\right]+\right. \\
& N_{3}\left[e^{\imath \vec{k}_{j} \vec{x}}, e^{\imath \vec{k}_{k} \vec{x}},\left(\hat{L}^{0}\right)^{-1} N_{3}\left[e^{-\imath \vec{k}_{k} \vec{x}}, e^{\imath \vec{k}_{i} \vec{x}}, e^{-\imath \vec{k}_{j} \vec{x}}\right]\right]+ \\
& N_{3}\left[e^{\imath \vec{k}_{k} \vec{x}}, e^{\imath \vec{k}_{j} \vec{x}},\left(\hat{L}^{0}\right)^{-1} N_{3}\left[e^{\imath \vec{k}_{i} \vec{x}}, e^{-\imath \vec{k}_{k} \vec{x}}, e^{-\imath \vec{k}_{j} \vec{x}}\right]\right]+ \\
& N_{3}\left[e^{\imath \vec{k}_{j} \vec{x}}, e^{\imath \vec{k}_{k} \vec{x}},\left(\hat{L}^{0}\right)^{-1} N_{3}\left[e^{\imath \vec{k}_{i} \vec{x}}, e^{-\imath \vec{k}_{k} \vec{x}}, e^{-\imath \vec{k}_{j} \vec{x}}\right]\right]+ \\
& N_{3}\left[e^{\imath \vec{k}_{k} \vec{x}}, e^{-\imath \vec{k}_{j} \vec{x}},\left(\hat{L}^{0}\right)^{-1} N_{3}\left[e^{-\imath \vec{k}_{k} \vec{x}}, e^{\imath \vec{k}_{i} \vec{x}}, e^{\imath \vec{k}_{j} \vec{x}}\right]\right]+ \\
& N_{3}\left[e^{-\imath \vec{k}_{j} \vec{x}}, e^{\imath \vec{k}_{k} \vec{x}},\left(\hat{L}^{0}\right)^{-1} N_{3}\left[e^{-\imath \vec{k}_{k} \vec{x}}, e^{\imath \vec{k}_{i} \vec{x}}, e^{\imath \vec{k}_{j} \vec{x}}\right]\right]+ \\
& N_{3}\left[e^{\imath \vec{k}_{k} \vec{x}}, e^{-\imath \vec{k}_{j} \vec{x}},\left(\hat{L}^{0}\right)^{-1} N_{3}\left[e^{\imath \vec{k}_{i} \vec{x}}, e^{-\imath \vec{k}_{k} \vec{x}}, e^{\imath \vec{k}_{j} \vec{x}}\right]\right]+ \\
& N_{3}\left[e^{-\imath \vec{k}_{j} \vec{x}}, e^{\imath \vec{k}_{k} \vec{x}},\left(\hat{L}^{0}\right)^{-1} N_{3}\left[e^{\imath \vec{k}_{i} \vec{x}}, e^{-\imath \vec{k}_{k} \vec{x}}, e^{\imath \vec{k}_{j} \vec{x}}\right]\right]+ \\
& N_{3}\left[e^{\imath \vec{k}_{j} \vec{x}},\left(\hat{L}^{0}\right)^{-1} N_{3}\left[e^{\imath \vec{k}_{k} \vec{x}}, e^{-\imath \vec{k}_{j} \vec{x}}, e^{\imath \vec{k}_{i} \vec{x}}\right], e^{-\imath \vec{k}_{k} \vec{x}}\right]+ \\
& N_{3}\left[e^{\imath \vec{k}_{j} \vec{x}},\left(\hat{L}^{0}\right)^{-1} N_{3}\left[e^{-\imath \vec{k}_{j} \vec{x}}, e^{\imath \vec{k}_{k} \vec{x}}, e^{\imath \vec{k}_{i} \vec{x}}\right], e^{-\imath \vec{k}_{k} \vec{x}}\right]+ \\
& N_{3}\left[e^{-\imath \vec{k}_{j} \vec{x}},\left(\hat{L}^{0}\right)^{-1} N_{3}\left[e^{\imath \vec{k}_{k} \vec{x}}, e^{\imath \vec{k}_{j} \vec{x}}, e^{\imath \vec{k}_{i} \vec{x}}\right], e^{-\imath \vec{k}_{k} \vec{x}}\right]+ \\
& N_{3}\left[e^{-\imath \vec{k}_{j} \vec{x}},\left(\hat{L}^{0}\right)^{-1} N_{3}\left[e^{\imath \vec{k}_{j} \vec{x}}, e^{\imath \vec{k}_{k} \vec{x}}, e^{\imath \vec{k}_{i} \vec{x}}\right], e^{-\imath \vec{k}_{k} \vec{x}}\right]+ \\
& N_{3}\left[\left(\hat{L}^{0}\right)^{-1} N_{3}\left[e^{\imath \vec{k}_{k} \vec{x}}, e^{-\imath \vec{k}_{j} \vec{x}}, e^{\imath \vec{k}_{i} \vec{x}}\right], e^{\imath \vec{k}_{j} \vec{x}}, e^{-\imath \vec{k}_{k} \vec{x}}\right]+ \\
& N_{3}\left[\left(\hat{L}^{0}\right)^{-1} N_{3}\left[e^{-\imath \vec{k}_{j} \vec{x}}, e^{\imath \vec{k}_{k} \vec{x}}, e^{\imath \vec{k}_{i} \vec{x}}\right], e^{\imath \vec{k}_{j} \vec{x}}, e^{-\imath \vec{k}_{k} \vec{x}}\right]+ \\
& N_{3}\left[\left(\hat{L}^{0}\right)^{-1} N_{3}\left[e^{\imath \vec{k}_{k} \vec{x}}, e^{\imath \vec{k}_{j} \vec{x}}, e^{\imath \vec{k}_{i} \vec{x}}\right], e^{-\imath \vec{k}_{j} \vec{x}}, e^{-\imath \vec{k}_{k} \vec{x}}\right]+ \\
& \left.N_{3}\left[\left(\hat{L}^{0}\right)^{-1} N_{3}\left[e^{\imath \vec{k}_{j} \vec{x}}, e^{\imath \vec{k}_{k} \vec{x}}, e^{\imath \vec{k}_{i} \vec{x}}\right], e^{-\imath \vec{k}_{j} \vec{x}}, e^{-\imath \vec{k}_{k} \vec{x}}\right]\right) \\
& f_{i j j^{-}}=f_{i j j}\left(\vec{k}_{j} \rightarrow-\vec{k}_{j}\right), \\
& \tilde{f}_{i i i}=\tilde{f}_{i j j}=\tilde{f}_{i j i}=0 \\
& \tilde{f}_{i i j}=e^{-\imath \vec{k}_{i} \vec{x}}\left(N_{3}\left[e^{-\imath \vec{k}_{i} \vec{x}},\left(\hat{L}^{0}\right)^{-1} N_{3}\left[e^{\imath \vec{k}_{i} \vec{x}}, e^{\imath \vec{k}_{i} \vec{x}}, e^{-\imath \vec{k}_{j} \vec{x}}\right], e^{\imath \vec{k}_{j} \vec{x}}\right]+\right. \\
& N_{3}\left[e^{-\imath \vec{k}_{i} \vec{x}},\left(\hat{L}^{0}\right)^{-1} N_{3}\left[e^{\imath \vec{k}_{i} \vec{x}}, e^{\imath \vec{k}_{i} \vec{x}}, e^{\imath \vec{k}_{j} \vec{x}}\right], e^{-\imath \vec{k}_{j} \vec{x}}\right]+ \\
& N_{3}\left[\left(\hat{L}^{0}\right)^{-1} N_{3}\left[e^{\imath \vec{k}_{i} \vec{x}}, e^{\imath \vec{k}_{i} \vec{x}}, e^{-\imath \vec{k}_{j} \vec{x}}\right], e^{-\imath \vec{k}_{i} \vec{x}}, e^{\imath \vec{k}_{j} \vec{x}}\right]+ \\
& \left.N_{3}\left[\left(\hat{L}^{0}\right)^{-1} N_{3}\left[e^{\imath \vec{k}_{i} \vec{x}}, e^{i \vec{k}_{i} \vec{x}}, e^{i \vec{k}_{j} \vec{x}}\right], e^{-\imath \vec{k}_{i} \vec{x}}, e^{-\imath \vec{k}_{j} \vec{x}}\right]\right) \\
& \tilde{f}_{i j k}=e^{-\imath \vec{k}_{i} \vec{x}}\left(N_{3}\left[e^{i \vec{k}_{j} \vec{x}},\left(\hat{L}^{0}\right)^{-1} N_{3}\left[e^{-\imath \vec{k}_{j} \vec{x}}, e^{\imath \vec{k}_{i} \vec{x}}, e^{i \vec{k}_{k} \vec{x}}\right], e^{-\imath \vec{k}_{k} \vec{x}}\right]+\right. \\
& N_{3}\left[e^{\imath \vec{k}_{j} \vec{x}},\left(\hat{L}^{0}\right)^{-1} N_{3}\left[e^{\imath \vec{k}_{i} \vec{x}}, e^{-\imath \vec{k}_{j} \vec{x}}, e^{\imath \vec{k}_{k} \vec{x}}\right], e^{-\imath \vec{k}_{k} \vec{x}}\right]+
\end{aligned}
$$




$$
\begin{aligned}
& N_{3}\left[e^{\imath \vec{k}_{j} \vec{x}},\left(\hat{L}^{0}\right)^{-1} N_{3}\left[e^{-\imath \vec{k}_{j} \vec{x}}, e^{\imath \vec{k}_{i} \vec{x}}, e^{-\imath \vec{k}_{k} \vec{x}}\right], e^{\imath \vec{k}_{k} \vec{x}}\right]+ \\
& N_{3}\left[e^{\imath \vec{k}_{j} \vec{x}},\left(\hat{L}^{0}\right)^{-1} N_{3}\left[e^{\imath \vec{k}_{i} \vec{x}}, e^{-\imath \vec{k}_{j} \vec{x}}, e^{-\imath \vec{k}_{k} \vec{x}}\right], e^{\imath \vec{k}_{k} \vec{x}}\right]+ \\
& N_{3}\left[e^{-\imath \vec{k}_{j} \vec{x}},\left(\hat{L}^{0}\right)^{-1} N_{3}\left[e^{\imath \vec{k}_{j} \vec{x}}, e^{\imath \vec{k}_{i} \vec{x}}, e^{\imath \vec{k}_{k} \vec{x}}\right], e^{-\imath \vec{k}_{k} \vec{x}}\right]+ \\
& N_{3}\left[e^{-\imath \vec{k}_{j} \vec{x}},\left(\hat{L}^{0}\right)^{-1} N_{3}\left[e^{\imath \vec{k}_{i} \vec{x}}, e^{\imath \vec{k}_{j} \vec{x}}, e^{\imath \vec{k}_{k} \vec{x}}\right], e^{-\imath \vec{k}_{k} \vec{x}}\right]+ \\
& N_{3}\left[e^{-\imath \vec{k}_{j} \vec{x}},\left(\hat{L}^{0}\right)^{-1} N_{3}\left[e^{\imath \vec{k}_{j} \vec{x}}, e^{\imath \vec{k}_{i} \vec{x}}, e^{-\imath \vec{k}_{k} \vec{x}}\right], e^{\imath \vec{k}_{k} \vec{x}}\right]+ \\
& N_{3}\left[e^{-\imath \vec{k}_{j} \vec{x}},\left(\hat{L}^{0}\right)^{-1} N_{3}\left[e^{\imath \vec{k}_{i} \vec{x}}, e^{\imath \vec{k}_{j} \vec{x}}, e^{-\imath \vec{k}_{k} \vec{x}}\right], e^{\imath \vec{k}_{k} \vec{x}}\right]+ \\
& N_{3}\left[\left(\hat{L}^{0}\right)^{-1} N_{3}\left[e^{-\imath \vec{k}_{j} \vec{x}}, e^{\imath \vec{k}_{i} \vec{x}}, e^{\imath \vec{k}_{k} \vec{x}}\right], e^{\imath \vec{k}_{j} \vec{x}}, e^{-\imath \vec{k}_{k} \vec{x}}\right]+ \\
& N_{3}\left[\left(\hat{L}^{0}\right)^{-1} N_{3}\left[e^{\imath \vec{k}_{i} \vec{x}}, e^{-\imath \vec{k}_{j} \vec{x}}, e^{\imath \vec{k}_{k} \vec{x}}\right], e^{\imath \vec{k}_{j} \vec{x}}, e^{-\imath \vec{k}_{k} \vec{x}}\right]+ \\
& N_{3}\left[\left(\hat{L}^{0}\right)^{-1} N_{3}\left[e^{-\imath \vec{k}_{j} \vec{x}}, e^{\imath \vec{k}_{i} \vec{x}}, e^{-\imath \vec{k}_{k} \vec{x}}\right], e^{\imath \vec{k}_{j} \vec{x}}, e^{\imath \vec{k}_{k} \vec{x}}\right]+ \\
& N_{3}\left[\left(\hat{L}^{0}\right)^{-1} N_{3}\left[e^{\imath \vec{k}_{i} \vec{x}}, e^{-\imath \vec{k}_{j} \vec{x}}, e^{-\imath \vec{k}_{k} \vec{x}^{x}}\right], e^{\imath \vec{k}_{j} \vec{x}}, e^{\imath \vec{k}_{k} \vec{x}}\right]+ \\
& N_{3}\left[\left(\hat{L}^{0}\right)^{-1} N_{3}\left[e^{\imath \vec{k}_{j} \vec{x}}, e^{\imath \vec{k}_{i} \vec{x}}, e^{\imath \vec{k}_{k} \vec{x}}\right], e^{-\imath \vec{k}_{j} \vec{x}}, e^{-\imath \vec{k}_{k} \vec{x}}\right]+ \\
& N_{3}\left[\left(\hat{L}^{0}\right)^{-1} N_{3}\left[e^{\imath \vec{k}_{i} \vec{x}}, e^{\imath \vec{k}_{j} \vec{x}}, e^{\imath \vec{k}_{k} \vec{x}}\right], e^{-\imath \vec{k}_{j} \vec{x}}, e^{-\imath \vec{k}_{k} \vec{x}}\right]+ \\
& \left.N_{3}\left[\left(\hat{L}^{0}\right)^{-1} N_{3}\left[e^{\imath \vec{k}_{j} \vec{x}}, e^{\imath \vec{k}_{i} \vec{x}}, e^{\left.-\imath \vec{L}_{k}\right)^{0}}\right], e^{-1} N_{3}\left[e^{\imath \vec{k}_{i} \vec{x}}, e^{\imath \vec{k}_{j} \vec{x}}, e^{\imath \vec{k}_{k} \vec{x}}, e^{-\imath \vec{k}_{k} \vec{x}}\right], e^{-\imath \vec{k}_{j} \vec{x}}, e^{\imath \vec{k}_{k} \vec{x}}\right]\right)
\end{aligned}
$$




\section{Bibliography}

[1] A. J. Koch and H. Meinhardt, Biological pattern formation: From basic mechanisms to complex structures, Rev. Mod. Phys. 66(4) (1994).

[2] E. Bodenschatz, W. Pesch, and G. Ahlers, Recent Developments in Rayleigh-Bénard Convection, Annu. Rev. Fluid Mech. 32, 709-778 (2000).

[3] F. H. Busse, Non-linear properties of thermal convection, Rep. Prog. Phys. 41 (1978).

[4] M. C. Cross and P. C. Hohenberg, Pattern formation outside of equilibrium, Rev. Mod. Phys. 65, 851-1112 (1993).

[5] R. S. Bennink, V. Wong, A. M. Marino, D. L. Aronstein, and R. W. Boyd, Honeycomb pattern formation by Laser-Beam Filamentation in Atomic Sodium Vapor, Phys. Rev. Lett. 88(11), 113901 (2002).

[6] W. Zhang and J. Vinals, Pattern formation in weakly damped parametric surface waves, J. Fluid Mech. 336, 301-330 (1997).

[7] A. Kudrolli and J. P. Gollub, Patterns and spatiotemporal chaos in parametrically forced surface waves: a systematic survey at large aspect ratio, Physica D 97, 133-154 (1996).

[8] J. H. Kaas, R. J. Nelson, M. Sur, C. S. Lin, and M. M. Merzenich, Multiple representations of the body within the primary somatosensory cortex of primates, Science 204(4392), 521-523 (1979).

[9] J. F. Olsen, E. I. Knudsen, and S. D. Esterly, Neural maps of interaural time and intensity differences in the optic tectum of the barn owl, J. Neurosci. 9, 2591-2605 (1989).

[10] D. L. Adams, L. C. Sincich, and J. C. Horton, Complete Pattern of Ocular Dominance Columns in Human Primary Visual Cortex, J. Neurosci. 27(39), 10391-10403 (2007).

[11] E. Yacoub, N. Harel, and K. Ugurbil, High-field fMRI unveils orientation columns in humans, PNAS 105(30), 10607-10612 (2008).

[12] V. Braitenberg and A. Schütz, Cortex: Statistics and Geometry of Neuronal Connectivity, Springer, Berlin, 1998. 
[13] A. Soward, Bifurcation and stability of finite amplitude convection in a rotating layer, Physica D 14, 227-241 (1985).

[14] J. Lega, J. V. Moloney, and A. C. Newell, Swift-Hohenberg Equation for Lasers, Phys. Rev. Lett. 73, 2978 (1994).

[15] N. V. Swindale, A model for the formation of orientation columns, Proc. R. Soc. Lond. B 215, 211-230 (1982).

[16] M. Kaschube, Pattern Selection in the Visual Cortex, PhD thesis, Göttingen University, 2005.

[17] A. A. Koulakov and D. B. Chklovskii, Orientation Preference Patterns in Mammalian Visual Cortex: A Wire Length Minimization Approach, Neuron 29(2), 519-527 (2001).

[18] F. Wolf and T. Geisel, Spontaneous pinwheel annihilation during visual development, Nature 395, 73-78 (1998).

[19] H. Y. Lee, M. Yahyanejad, and M. Kardar, Symmetry considerations and development of pinwheels in visual maps, PNAS 100(26), 16036-16040 (2003).

[20] M. W. Cho and S. Kim, Understanding Visual Map Formation through Vortex Dynamics of Spin Hamiltonian Models, Phys. Rev. Lett. 92(1), 018101 (2004).

[21] N. V. Swindale, Coverage and the design of striate cortex, Biol. Cybern. 65, 415-424 (1991).

[22] N. V. Swindale, The development of topography in the visual cortex: A review of models, Network 7 (1996).

[23] N. V. Swindale, D. Shoham, A. Grinwald, T. Bonhoeffer, and M. Hübener, Visual cortex maps are optimized for uniform coverage, Nature Neuroscience 3, 822-826 (2000).

[24] N. V. Swindale, How different feature spaces may be represented in cortical maps, Network: Comput. Neural Syst. 15, 217-242 (2004).

[25] A. A. Koulakov and D. B. Chklovskii, Direction of motion maps in the visual cortex: a wire length minimization approach, Neurocomputing 44-46, 489-494 (2002).

[26] D. H. Hubel and T. N. Wiesel, Functional Architecture of Macaque Monkey Visual Cortex, Proc. R. Soc. London 198, 1-59 (1977).

[27] F. Hoffsümmer, F. Wolf, T. Geisel, S. Löwel, and K. E. Schmidt, Sequential Bifurcation and Dynamical Rearrangement of Columnar Patterns during Cortical Development, Comput. Neural. Syst. (1996).

[28] G. J. Goodhill and A. Cimponeriu, Analysis of the elastic net model applied to the formation of ocular dominance and orientation columns, Comput. Neural. Syst. 11, 153-168 (2000). 
[29] M. A. Carreira-Perpinan, R. J. Lister, and G. J. Goodhill, A Computational Model for the Development of Multiple Maps in Primary Visual Cortex, Cereb. Cortex 15, $1222-1233$ (2005).

[30] H. Yu, B. J. Farley, D. Z. Jin, and M. Sur, The Coordinated Mapping of Visual Space and Response Features in Visual Cortex, Neuron 47, 267-280 (2005).

[31] E. Erwin, K. Obermayer, and K. Schulten, Models of Orientation and Ocular Dominance Columns in the Visual Cortex: A Critical Comparison, Neural Computation 7(3), 425468 (1995).

[32] K. Obermayer, G. G. Blasdel, and K. Schulten, Statistical-mechanical analysis of selforganization and pattern formation during the development of visual maps, Phys. Rev. A 45(10), 7568-7589 (1992).

[33] B. J. Farley, H. Yu, D. Z. Jin, and M. Sur, Alteration of Visual Input Results in a Coordinated Reorganization of Multiple Visual Cortex Maps, J. Neurosci. 27, 1029910310 (2007).

[34] N. V. Swindale, How many maps are there in visual cortex?, Cereb. Cortex 10, 633-634 (2000).

[35] J. A. Bednar and R. Miikkulainen, Joint maps for orientation, eye, and direction preference in a self-organizing model of V1, Neurocomputing 69(10-12), 1272-1276 (2006).

[36] H. Nakagama, T. Tani, and S. Tanaka, Theoretical and experimental studies of relationship between pinwheel centers and ocular dominance columns in the visual cortex, Neuroscience Research 55(4), 370-382 (2006).

[37] S. Grossberg and S. J. Olson, Rules for the Cortical Map of Ocular Dominance and Orientation Columns, Neural Networks 7, 883-894 (1994).

[38] D. M. Pierre, Modeling Orientation and Ocular Dominance Columns in the Visual Cortex, PhD thesis, MIT, 1997.

[39] W. H. Bosking, Y. Zhang, B. Schofield, and D. Fitzpatrick, Orientation selectivity and the arrangement of horizontal connections in tree shrew striate cortex, J. Neurosci. 17(6), 2112-2127 (1997).

[40] K. S. Rockland and J. S. Lund, Widespread periodic intrinsic connections in the tree shrew visual cortex, Science 215, 1532-1534 (1982).

[41] L. C. Sincich and G. G. Blasdel, Oriented Axon Projections in Primary Visual Cortex of the Monkey, J. Neurosci. 21(12), 4416-4428 (2001). 
[42] L. E. White, D. M. Coppola, and D. Fitzpatrick, The contribution of sensory experience to the maturation of orientation selectivity in ferret visual cortex, Nature 411, 1049 (2001).

[43] M. Weliky and L. C. Katz, Functional mapping of horizontal connections in developing ferret visual cortex: experiments and modeling, J. Neurosci. 14, 7291-7305 (1994).

[44] C. D. Gilbert and T. N. Wiesel, Morphology and intracortical projections of functionally characterized neurons in the cat visual cortex, Nature 280 (1979).

[45] C. D. Gilbert and T. N. Wiesel, Clustered intrinsic connections in cat visual cortex, J. Neurosci. 3, 1116-1133 (1983).

[46] Z. F. Kisvarday, E. Toth, M. Rausch, and U. T. Eysel, Orientation-specific relationship between populations of excitatory and inhibitory lateral connections in the visual cortex of the cat, Cerebral Cortex 7, 605-618 (1997).

[47] E. M. Callaway and L. C. Katz, Emergence and Refinement of Clustered Horizontal Connections in Cat Striate Cortex, J. Neurosci. 10(4), 1134-1153 (1990).

[48] S. Löwel and W. Singer, Selection of intrinsic horizontal connections in the visual cortex by correlated neuronal activity, Science 255, 209-212 (1992).

[49] F. Wolf, Symmetry, Multistability and Long-Range Interactions in Brain Development, Phys. Rev. Lett. 95, 208701 (2005).

[50] F. Wolf, Les houches 2003 lecture notes. Methods and Models in neurophysics, Elsevier, Amsterdam, 2005.

[51] D. Hannula, D. Simons, and N. J. Cohen, Imaging implicit perception: promise and pitfalls, Nature Reviews Neuroscience 6 (2005).

[52] O. Creutzfeldt, Cortex Cerebri: Performance, structural and functional organization of the cortex, Oxford University Press, 1995.

[53] S. LeVay and S. B. Nelson, Columnar organization of the visual cortex, in Vision and Visual Dysfunction, pages 266-315, Macmillan, 1991.

[54] A. Das, Cortical Maps: Where theory meets experiments, Neuron 47, 168-171 (2005).

[55] M. Kaschube, F. Wolf, M. Puhlmann, S. Rathjen, K. E. Schmidt, T. Geisel, and S. Löwel, The pattern of ocular dominance columns in cat primary visual cortex: Intra- and interindividual variability of column spacing and its dependence on genetic background, Eur. J. Neurosci. 18, 3251-3266 (2003.).

[56] J. C. Horton and D. R. Hocking, Intrinsic Variability of Ocular Dominance Column Periodicity in Normal Macaque Monkeys, J. Neurosci. 16, 7228-7339 (1996). 
[57] N. P. Issa, J. T. Trachtenberg, B. Chapman, K. R. Zahs, and M. P. Stryker, The Critical Period for Ocular Dominance Plasticity in the Ferret's Visual Cortex, J. Neurosci. 19(16), 6965-6978 (1999).

[58] M. C. Crair, D. C. Gillespie, and M. P. Stryker, The Role of Visual Experience in the Development of Columns in Cat Visual Cortex, Science 279, 556-570 (1998).

[59] M. Y. Frenkel and M. F. Bear, How Monocular Deprivation Shifts Ocular Dominance in Visual Cortex of Young Mice, Neuron 44, 917-923 (2004).

[60] K. Ohki, S. Chung, P. Kara, M. Hübener, T. Bonhoeffer, and R. C. Reid, Highly ordered arrangement of single neurons in orientation pinwheels, Nature 442(24), 925 (2006).

[61] N. D. Mermin, The topological theory of defects in ordered media, Rev. Mod. Phys. $\mathbf{5 1}(3)$ (1979).

[62] K. Obermayer and G. G. Blasdel, Singularities in Primate Orientation Maps, Neural Computation 9, 555-575 (1997).

[63] S. C. Rao, L. J. Toth, and M. Sur, Optically imaged maps of orientation preference in primary visual cortex of cats and ferrets, J. Comp. Neurol. 387, 358-370 (1997).

[64] T. Müller, M. Stetter, M. Hübener, F. Sengpiel, T. Bonhoeffer, I. Gödeke, B. Chapman, S. Löwel, and K. Obermayer, An Analysis of Orientation and Ocular Dominance Patterns in the Visual Cortex of Cats and Ferrets, Neural Computation 12, 2573-2595 (2000).

[65] A. Shmuel and A. Grinwald, Functional Organization for Direction of Motion and Its Relationship to Orientation Maps in Cat Area 18, J. Neurosci. 16(21), 6945-6964 (1996).

[66] Y. Li, D. Fitzpatrick, and L. E. White, The development of direction selectivity in ferret visual cortex requires early visual experience, Nature Neuroscience 9(5) (2006).

[67] N. P. Issa, C. Trepel, and M. P. Stryker, Spatial Frequency Maps in Cat Visual Cortex, J. Neurosci. 15, 8504-8514 (2000).

[68] M. Hübener, D. Shoham, A. Grinwald, and T. Bonhoeffer, Spatial Relationships among Three Columnar Systems in Cat Area 17, J. Neurosci. 17(23), 9270-9284 (1997).

[69] K. E. Schmidt, D.-S. Kim, W. Singer, T. Bonhoeffer, and S. Löwel, Functional Specificity of Long-Range Intrinsic and Interhemispheric Connections in the Visual Cortex of Strabismic Cats, J. Neurosci. 17(14), 5480-5492 (1997).

[70] J. Matsubara, M. Cynader, and N. V. Swindale, Anatomical properties and physiological correlates of the intrinsic connections in cat area 18, J. Neurosci. 7, 1428-1446 (1987). 
[71] T. Yoshioka, G. G. Blasdel, J. B. Levitt, and J. S. Lund, Relation between Patterns of Intrinsic Lateral Connectivity, Ocular Dominance, and Cytochrome Oxidase-Reactive Regions in Macaque Monkey Striate Cortex, Cerebral Cortex 6, 297-310 (1996).

[72] R. Malach, Y. Amir, M. Harel, and A. Grinwald, Relationship between intrinsic connections and functional architecture revealed by optical imaging and in vivo targeted biocytin injections in primate striate cortex, Proc. Natl. Acad. Sci. U.S.A. 90, 1046910473 (1993).

[73] S. Löwel, K. E. Schmidt, D.-S. Kim, F. Wolf, F. Hoffsümmer, W. Singer, and T. Bonhoeffer, The layout of orientation and ocular dominance domains in area 17 of strabismic cats, Eur. J. Neurosci. 10, 2629-2643 (1998).

[74] M. C. Crair, E. S. Ruthazer, D. C. Gillespie, and M. P. Stryker, Ocular Dominance Peaks at Pinwheel Center Singularities of the Orientation Map in Cat Visual Cortex, J. Neurophysiol. 77, 3381-3385 (1997).

[75] K. Obermayer and G. G. Blasdel, Geometry of orientation and ocular dominance columns in monkey striate cortex, J. Neurosci. 13, 4114-4129 (1993).

[76] S. D. Van Hooser, A. J. Heimel, S. Chung, S. B. Nelson, and L. J. Toth, Orientation Selectivity without Orientation Maps in Visual Cortex of a Highly Visual Mammal, J. Neurosci. 25(1), 19-28 (2005).

[77] J. C. Horton and D. R. Hocking, Anatomical Demonstration of Ocular Dominance Columns in Striate Cortex of the Squirrel Monkey, J. Neurosci. 16(17), 5510-5522 (1996).

[78] D. L. Adams and J. C. Horton, Capricious expression of cortical columns in the primate brain, Nature Neuroscience 6(2) (2003).

[79] M. Kaschube, F. Wolf, T. Geisel, and S. Löwel, Genetic Influence on Quantitative Features of Neocortical Architecture, J. Neurosci. 22(16), 7206-7217 (2002).

[80] S. LeVay, M. Connolly, and D. C. Van Essen, The complete pattern of ocular dominance stripes in the striate cortex and visual field of the macaque monkey, J. Neurosci. 5, 486-501 (1985).

[81] M. Sur and C. A. Leamey, Development and Plasticity of cortical areas and networks, Nature Reviews Neuroscience 2, 251 (2001).

[82] L. E. White and D. Fitzpatrick, Vision and Cortical Map Development, Neuron 56(2), $327-338$ (2007).

[83] J. C. Crowley and L. C. Katz, Early Development of Ocular Dominance Columns, Science 290(5495), 1321-1324 (2000). 
[84] F. Sengpiel, P. Stawinski, and T. Bonhoeffer, Influence of experience on orientation maps in cat visual cortex, Nature Neuroscience 2(8) (1999).

[85] B. Chapman, M. D. Jacobson, H. O. Reiter, and M. P. Stryker, Ocular dominance shift in kitten visual cortex caused by imbalance in retinal electrical activity, Nature 324, 154-156 (1986).

[86] M. I. Law and M. Constantine-Paton, Eye-Specific Termination Bands in Tecta of Three-Eyed Frogs, Science 202, 639-641 (1978).

[87] J. Sharma, A. Angelucci, and M. Sur, Induction of visual orientation modules in auditory cortex, Nature 404, 841-847 (2000).

[88] B. Godde, R. Leonhardt, S. M. Cords, and H. R. Dinse, Plasticity of Orientation preference maps in the visual cortex of adult cats, PNAS 99(9) (2002).

[89] B. Chapman, M. P. Stryker, and T. Bonhoeffer, Development of Orientation Preference Maps in Ferret Primary Visual Cortex, J. Neurosci. 16(20), 6443-6453 (1996).

[90] J. C. Horton and D. R. Hocking, An adult-like pattern of ocular dominance columns in striate cortex of newborn monkeys prior to visual experience, J. Neurosci. 16, 1791-1807 (1996).

[91] T. N. Wiesel and D. H. Hubel, Ordered arrangement of orientation columns in monkeys lacking visual experience, J. Comp. Neurol. 158(3), 307-318 (1974).

[92] G. G. Blasdel, K. Obermayer, and L. Kiorpes, Organization of ocular dominance and orientation columns in the striate cortex of neonatal macaque monkeys, Visual Neuroscience 12, 589-603 (1995).

[93] S. LeVay, M. P. Stryker, and C. J. Shatz, Ocular dominance columns and their development in layer IV of the cat's visual cortex: A quantitative study, J. Comp. Neurol. 179, 223-244 (1978).

[94] F. Wolf, K. Pawelzik, O. Scherf, T. Geisel, and S. Löwel, How can squint change the spacing of ocular dominance columns?, J. Physiol. 94, 525-537 (2000).

[95] J. B. Swift and P. C. Hohenberg, Hydrodynamic fluctuations at the convective instability, Phys. Rev. A 15, 319-328 (1977).

[96] M. Schnabel, A Symmetry of the Visual World in the Architecture of the Visual Cortex, $\mathrm{PhD}$ thesis, Göttingen University, 2008.

[97] M. C. Cross and H. Greenside, Pattern Formation and Dynamics in Nonequilibrium Systems, Cambride University Press, 2009.

[98] V. Braitenberg and C. Braitenberg, Geometry of Orientation Columns in the Visual Cortex, Biol. Cybern. (33), 179-186 (1979). 
[99] M. W. Cho and S. Kim, Different Ocular Dominance Map Formation Influenced by Orientation Preference Columns in Visual Cortices, Phys. Rev. Lett. 94, 068701 (2005).

[100] M. Huang, Spatio-Temporal Dynamics of Pattern Formation in the Cerebral Cortex, PhD thesis, Göttingen University, 2009.

[101] A. Brabska-Barwinska and C. von der Malsburg, Establishment of a Scaffold for Orientation Maps in Primary Visual Cortex of Higher Mammals, J. Neurosci. 28(1), 249-257 (2008).

[102] R. Durbin and G. J. Mitchison, A dimension reduction framework for understanding cortical maps, Nature 343, 644-647 (1990).

[103] A. Das and C. D. Gilbert, Distortions of visuotopic map match orientation singularities in primary visual cortex, Nature 387, 594-598 (1997).

[104] G. J. Mitchison and N. V. Swindale, Can Hebbian Volume Learning Explain Discontinuities in Cortical Maps?, Neural Computation 11, 1519-1526 (1999).

[105] R. M. Gray, Toeplitz and Circulant matrices: A review, Communications and Information Theory 2(3), 155-239 (2005).

[106] R. Lifshitz and D. M. Petrich, Theoretical Model for Faraday Waves with MultipleFrequency Forcing, Phys. Rev. Lett. 79(7), 1261-1264 (1997).

[107] J. C. Crowley and L. C. Katz, Development of ocular dominance columns in the absence of retinal input, Nature Neuroscience 2, 1125-30 (1999).

[108] E. Bartfeld and A. Grinwald, Relationships between orientation-preference pinwheels, cytochrome oxidase blobs, and ocular-dominance columns in primate striate cortex, PNAS 89(24), 11905-11909 (1992).

[109] R. Engelmann, J. M. Crook, and S. Löwel, Optical imaging of orientation and ocular dominance maps in area 17 of cats with convergent strabismus, Visual Neuroscience 19, 39-49 (2002).

[110] L. E. White, W. H. Bosking, and D. Fitzpatrick, Consistent mapping of orientation preference across irregular functional domains in ferret visual cortex, Visual Neuroscience 18, 65-76 (2001).

[111] Y. Matsuda, K. Ohki, T. Saito, A. Ajima, and D.-S. Kim, Coincidence of ipsilateral ocular dominance peaks with orientation pinwheel centers in cat visual cortex, NeuroReport 11(15), 3337-3343 (2000).

[112] F. Hoffsümmer, F. Wolf, T. Geisel, S. Löwel, and K. E. Schmidt, Sequential bifurcation of orientation- and ocular dominance maps, page 535, Paris, 1995, EC2 \& Cie. 
[113] F. Hoffsümmer, F. Wolf, T. Geisel, K. E. Schmidt, and S. Löwel, Sequential emergence of orientation- and ocular dominance maps, in Learning and Memory, Proceedings of the 23rd Göttingen Neurobiology Conference 1995, edited by N. Elsner and R. Menzel, page 97, Stutttgart, 1997, Thieme Verlag.

[114] M. I. Law and M. Constantine-Paton, Anatomy and Physiology of Experimentally Produced Striped Tecta, J. Neurosci. 1, 741-759 (1981).

[115] D. G. Jones, R. C. Van Sluyters, and K. M. Murphy, A Computational Model for the Overall Pattern of Ocular Dominance, J. Neurosci. 11(12), 3794-3808 (1991).

[116] D. Heide, Nonlinear Dynamics of Large Scale Patterns in the Visual Cortex, Master's thesis, University Goettingen, 2005.

[117] B. A. Malomed, A. A. Nepomnyashchy, and M. I. Tribelsky, Two-dimensional quasiperiodic structures in nonequilibrium systems, Zh. Eksp. Teor. Fiz. 96, 684-700 (1989).

[118] A. M. Rucklidge and W. J. Rucklidge, Convergence properties of the 8,10 and 12 mode representations of quasipatterns, Physica D 178, 62-82 (2003).

[119] F. Wolf, Strukturbildung in der Entwicklung des visuellen Kortex, PhD thesis, Goethe University, Frankfurt, 1999.

[120] P. Mannevile, Dissipative Structures and Weak Turbulence, Academic Press, 1990.

[121] L. Reichl, S. Löwel, and F. Wolf, Pinwheel stabilization by ocular dominance segregation, Phys. Rev. Lett. 102, 208101 (2009).

[122] G. Afshar, Simulation and Analysis of Neuronal Pattern Formation in the Visual Cortex, Master's thesis, Göttingen University, 2009.

[123] S. Löwel and W. Singer, Tangential intracortical pathways and the development of iso-orientation bands in cat striate cortex, Brain Research 56(1), 99-106 (1990).

[124] M. C. Cross and A. C. Newell, Convection patterns in large aspect ratio systems, Physica D 10, 299-328 (1984).

[125] P. N. Brown and Y. Saad, Hybrid Krylov Methods for Nonlinear Systems of Equations, J. Sci. and Stat. Comput. 11, 450-481 (1990).

[126] D. J. E. and R. B. Schnabel, Numerical Methods for Unconstrained Optimization and Nonlinear Equations, Prentice-Hall, 1983.

[127] S. Balay, K. Buschelman, W. D. Gropp, D. Kaushik, M. G. Knepley, L. C. McInnes, B. F. Smith, and H. Zhang, PETSc Web page, 2001.

[128] W. H. Press, Numerical recipes in C: The art of scientific computing, Cambridge University Press, 1992. 


\section{Acknowledgments}

After all those years, there is a long list of people who contributed in some way to this thesis, for which I would like to express thanks.

I thank Theo Geisel for giving me the opportunity to work in his group. He provided excellent working conditions and heads an vigorous institute with a great atmosphere. I would like to thank my supervisor Fred Wolf. He provided me with many helpful suggestions, important advice and gave me the freedom to pursue own ideas. His enthusiasm on the project often helped me to overcome doubts and frustration.

I also wish to thank Siegrid Löwel for helpful suggestions and many biological insights. I would like to thank the map folks: Ghazaleh Afshar, Matthias Kaschube, Michael Schnabel, Min Huang, and Wolfgang Keil. In particular, I would like to thank Dominik Heide for his constant help with numerical problems. I thank Dmitry Tsigankov for interesting discussions and re-establishing the 'map club'.

I thank Oliver Bendix with whom I shared a room for all these years. Oliver has always a helping hand and found quick solutions whenever I felt in trouble with linux. Owing to him I learned a lot of science beyond the borders of my own work.

Denny Fliegner and Yorck-Fabian Beensen provided me assistance in numerous computer problems.

I thank Corinna Trautsch, Katharina Jeremias, Regina Wunderlich, Tanja Gindele, and Tobias Niemann for management and support.

I thank Michelle Monteforte and Hecke Degering for critically reading the manuscript. Thanks to all the members of the Department of Nonlinear Dynamics.

I owe many thanks to friends for their patience in the past years.

I would like to thank my family for their constant support.

Finally, I want to thank Jasmin Held. Without her understanding, help, and encouragement this study would not have been completed. 


\section{Curriculum vitae}

\section{Persönliche Daten}

Name:

Geburtsdatum:

Geburtsort:

Staatsangehörigkeit:

Familienstand:

\section{Ausbildung}

$6 / 1997$

10/1998-12/2003

$10 / 2000$

$12 / 2002-12 / 2003$

$12 / 2003$

seit $05 / 2004$

seit $04 / 2005$
Lars Reichl

26. Oktober 1976

Sinsheim

deutsch

ledig

Studium der Physik,

Ruprecht-Karls-Universität, Heidelberg

Vordiplom in Physik

Diplomarbeit, Institut für theoretische Physik, Ruprecht-Karls-Universität, Heidelberg

Titel der Arbeit: "Composite gauge fields and localization in extra dimensions"

Diplom in Physik

Wissenschaftlicher Mitarbeiter am Max Planck Institut für Dynamik und Selbstorganisation, Göttingen

Promotionsstudium, Institut für Nichtlineare Dynamik, Georg-August-Universität, Göttingen

Göttingen, 5. April 2010 